\title{
Internal Differentiation, Comparative Variability, Structural Morphology, Normative Aspect Of Prognostication Of Ipse Dixit Np Hard Problems-A Totalistic Paradigmatic Statement
}

\author{
${ }^{1}$ DR K N PRASANNA KUMAR, ${ }^{2}$ PROF B S KIRANAGI AND \\ ${ }^{3}$ PROF C S BAGEWADI \\ ${ }^{1}$ Post doctoral researcher, Dr KNP Kumar has three PhD's, one each in Mathematics, Economics and \\ Political science and a D.Litt. in Political Science, Department of studies in Mathematics, Kuvempu \\ University, Shimoga, Karnataka, India \\ ${ }^{2}$ UGC Emeritus Professor (Department of studies in Mathematics), Manasagangotri, University of \\ Mysore, Karnataka, India \\ ${ }^{3}$ Chairman, Department of studies in Mathematics and Computer science, Jnanasahyadri Kuvempu \\ university, Shankarghatta, Shimoga district, Karnataka, India
}

\begin{abstract}
We develop a progressive comparable to Bank's General Ledger, and call it a General Theory of all the problems under the head of NP hard problems. Problems have their variables. For instance "Travelling Sales man problem" can have various different cities. Based upon parameters classification is done and stability analysis, asymptotic stability and Solutional behaviour of the equations are investigated We eschew from stating any primary predications, coextensive representations, predicational anteriority of the Problems attributed to space constraints. In consideration to the parametric based classification and there is both ontological consonance, primordial exactitude, and phenomenological testimony as one finds in Bank's ledgers. General Ledger is in fact the statement of all inflows and outflows, such a one as that occurs in problems, and theories in some conditions, like for example the conservation of energy breaking down in Hawking's radiation. Emphasis is laid on the fact that for instance a travelling salesman makes some move and then retracts to redress his move or starts another move to further his final destination. And this destination is General Ledger.-The General Theory Of all the NP (hard) problems. .It is a journey, a journey to find the final balance which probably never ends like an account never closes. So we are on to the journey
\end{abstract}

KEY WORDS: Boolean satisfiability Problem, N Puzzle, Knapsack Problem, Hamiltonian Path problem, Travelling Salesman Problem

\section{INTRODUCTION:}

As stated in the abstract we will not give any introduction, inconsideration to the leviathans' material and humungous literature on each subject matter for fear of missing woods for trees. On the other hand, for the interested reader the literature provides a rich receptacle, repository and treasure-trove of knowledge, And also because of space constraints. We note that the NP (HARD) problems are classified as follows:

1) Boolean satisfiability Problem

2) N Puzzle

3) Knapsack Problem

4) Hamiltonian Path problem

5) Travelling Salesman Problem

6) Sub graph Isomorphism Problem

7) Subset Sum problem

8) Clique Problem

9) Vertex Cover Problem

10) Independent Set problem

11) Dominating set problem.

12) Graph Coloring Problem

As in a Bank, various parameters are there for an account like balance standing, rate of interest, 
implications of inflation, money depression, depreciation of the currency, implications of Policies, philosophies and programs of the Government, each problem has certain parameters. That Gravity is constant does not mean it does not depend upon the masses of individual particles and there is no total gravity. Stratification is done based on the parameters of each problem and then consummated with the other to form a monolithic Diaspora for building the Model, which essentially as said is a progressive, nay a General Theory Of all the ways and means in which the problem can be solved be it by invocation or by abnegation and revocation of the action. Everything is recorded in the Computer and we draw up a Final General Ledger-nay The General Theory Of all NP (HARD) Problems. Essentially a prediction model, it as said analyses various other facets too.

\section{GLOSSARY OF THE SYSTEM BOOLEAN SATISFIABILITY PROBLEM AND N PUZZLE \\ NOTATION :}

$G_{13}:$ Category One Of Boolean Satisfiability Problem

$G_{14}$ : Category Two Of Boolean Satisfiability Problem

$G_{15}:$ Category Three Of Boolean Satisfiability Problem

$T_{13}$ : Category One Of N Puzzle

$T_{14}$ :Category Two Of N Puzzle

$T_{15}$ :Category Three Of N Puzzle

\section{GLOSSARY OF THE SYSTEM KNAPSACK PROBLEM AND HAMILTONIAN PATH PROBLEM:}

$G_{16}:$ Category One Of Knapsack Problem

$G_{17}:$ Category Two Of Knapsack Problem

$G_{18}:$ Category Three Of Knapsack Problem

$T_{16}$ : Category One Of Hamiltonian Path Problem

$T_{17}$ : Category Two Of Hamiltonian Path Problem

$T_{18}:$ Category Three Of Hamiltonian Path Problem

\section{GLOSSARY OF THE SYSTEM:TRAVELLING SALESMAN PROBLEM AND SUBGRAPH ISOMERISM PROBLEM}

$G_{20}:$ Category One Of Travelling Salesman Problem

$G_{21}:$ Category Two Of Travelling Salesman Problem

$G_{22}:$ Category Three Of Travelling Salesman Problem

$T_{20}:$ Category One Of Sub graph Isomerism Problem 
Internal Differentiation, Comparative Variability, Structural Morphology, Normative Aspect Of Prognostication Of Ipse Dixit Np Hard Problems-A Totalistic Paradigmatic Statement

$T_{21}$ : Category Two Of Sub graph Isomerism Problem

$T_{22}:$ Category Three Of Sub graph Isomerism Problem

\section{GLOSSARY FOR THE SYSTEM: SUBSET SUM PROBLEM AND CLIQUE PROBLEM}

$G_{24}:$ Category One Of Subset Sum Problem

$G_{25}:$ Category Two Of Subset Sum Problem

$G_{26}:$ Category Three Of Subset Sum Problem

$T_{24}$ : Category One Of Clique Problem

$T_{25}$ : Category Two Of Clique Problem

$T_{26}$ : Category Three Of Clique Problem

GLOSSARY FOR THE SYSTEM: VERTEX COVER PROBLEM AND INDEPENDENT SET PROBLEM

$G_{28}:$ Category One Of Vertex Cover Problem

$G_{29}:$ Category Two Of Vertex Cover Problem

$G_{30}:$ Category Three Of Vertex Cover Problem

$T_{28}:$ Category One Of Independent Set Problem

$T_{29}:$ Category Two Of Independent Set Problem

$T_{30}:$ Category Three Of Independent Set Problem

\section{GLOSSARY OF THE SYSTEM: DOMINATING SET PROBLEM AND GRAPH COLORING} PROBLEM

Dominating Set Problem

$G_{33}$ : Category Two Of Dominating Set Problem

$G_{34}$ : Category Three Of Dominating Set Problem

$T_{32}$ : Category One Of Graph Coloring Problem

$T_{33}$ : Category Two Of Graph Coloring Problem

$T_{34}$ : Category Three Of Graph Coloring Problem 


\section{ACCENTUATION COEFFICIENTS}

$$
\begin{aligned}
& \left(a_{13}\right)^{(1)},\left(a_{14}\right)^{(1)},\left(a_{15}\right)^{(1)},\left(b_{13}\right)^{(1)},\left(b_{14}\right)^{(1)},\left(b_{15}\right)^{(1)}\left(a_{16}\right)^{(2)},\left(a_{17}\right)^{(2)},\left(a_{18}\right)^{(2)} \\
& \left(b_{16}\right)^{(2)},\left(b_{17}\right)^{(2)},\left(b_{18}\right)^{(2)}:\left(a_{20}\right)^{(3)},\left(a_{21}\right)^{(3)},\left(a_{22}\right)^{(3)},\left(b_{20}\right)^{(3)},\left(b_{21}\right)^{(3)},\left(b_{22}\right)^{(3)} \\
& \left(a_{24}\right)^{(4)},\left(a_{25}\right)^{(4)},\left(a_{26}\right)^{(4)},\left(b_{24}\right)^{(4)},\left(b_{25}\right)^{(4)},\left(b_{26}\right)^{(4)},\left(b_{28}\right)^{(5)},\left(b_{29}\right)^{(5)},\left(b_{30}\right)^{(5)}, \\
& \left(a_{28}\right)^{(5)},\left(a_{29}\right)^{(5)},\left(a_{30}\right)^{(5)},\left(a_{32}\right)^{(6)},\left(a_{33}\right)^{(6)},\left(a_{34}\right)^{(6)},\left(b_{32}\right)^{(6)},\left(b_{33}\right)^{(6)},\left(b_{34}\right)^{(6)}
\end{aligned}
$$

\section{DISSIPATION COEFFCIENTS}

$$
\begin{aligned}
& \left(a_{13}^{\prime}\right)^{(1)},\left(a_{14}^{\prime}\right)^{(1)},\left(a_{15}^{\prime}\right)^{(1)},\left(b_{13}^{\prime}\right)^{(1)},\left(b_{14}^{\prime}\right)^{(1)},\left(b_{15}^{\prime}\right)^{(1)},\left(a_{16}^{\prime}\right)^{(2)},\left(a_{17}^{\prime}\right)^{(2)},\left(a_{18}^{\prime}\right)^{(2)}, \\
& \left(b_{16}^{\prime}\right)^{(2)},\left(b_{17}^{\prime}\right)^{(2)},\left(b_{18}^{\prime}\right)^{(2)},\left(a_{20}^{\prime}\right)^{(3)},\left(a_{21}^{\prime}\right)^{(3)},\left(a_{22}^{\prime}\right)^{(3)},\left(b_{20}^{\prime}\right)^{(3)},\left(b_{21}^{\prime}\right)^{(3)},\left(b_{22}^{\prime}\right)^{(3)} \\
& \left(a_{24}^{\prime}\right)^{(4)},\left(a_{25}^{\prime}\right)^{(4)},\left(a_{26}^{\prime}\right)^{(4)},\left(b_{24}^{\prime}\right)^{(4)},\left(b_{25}^{\prime}\right)^{(4)},\left(b_{26}^{\prime}\right)^{(4)},\left(b_{28}^{\prime}\right)^{(5)},\left(b_{29}^{\prime}\right)^{(5)},\left(b_{30}^{\prime}\right)^{(5)} \\
& \left(a_{28}^{\prime}\right)^{(5)},\left(a_{29}^{\prime}\right)^{(5)},\left(a_{30}^{\prime}\right)^{(5)},\left(a_{32}^{\prime}\right)^{(6)},\left(a_{33}^{\prime}\right)^{(6)},\left(a_{34}^{\prime}\right)^{(6)},\left(b_{32}^{\prime}\right)^{(6)},\left(b_{33}^{\prime}\right)^{(6)},\left(b_{34}^{\prime}\right)^{(6)}
\end{aligned}
$$

\section{GOVERNING EQUATIONS OF THE SYSTEM BOOLEAN SATISFIABILITY PROBLEM} AND N PUZZLE

The differential system of this model is now

$$
\begin{aligned}
& \frac{d G_{13}}{d t}=\left(a_{13}\right)^{(1)} G_{14}-\left[\left(a_{13}^{\prime}\right)^{(1)}+\left(a_{13}^{\prime \prime}\right)^{(1)}\left(T_{14}, t\right)\right] G_{13} \\
& \frac{d G_{14}}{d t}=\left(a_{14}\right)^{(1)} G_{13}-\left[\left(a_{14}^{\prime}\right)^{(1)}+\left(a_{14}^{\prime \prime}\right)^{(1)}\left(T_{14}, t\right)\right] G_{14} \\
& \frac{d G_{15}}{d t}=\left(a_{15}\right)^{(1)} G_{14}-\left[\left(a_{15}^{\prime}\right)^{(1)}+\left(a_{15}^{\prime \prime}\right)^{(1)}\left(T_{14}, t\right)\right] G_{15} \\
& \frac{d T_{13}}{d t}=\left(b_{13}\right)^{(1)} T_{14}-\left[\left(b_{13}^{\prime}\right)^{(1)}-\left(b_{13}^{\prime \prime}\right)^{(1)}(G, t)\right] T_{13} \\
& \frac{d T_{14}}{d t}=\left(b_{14}\right)^{(1)} T_{13}-\left[\left(b_{14}^{\prime}\right)^{(1)}-\left(b_{14}^{\prime \prime}\right)^{(1)}(G, t)\right] T_{14} \\
& \frac{d T_{15}}{d t}=\left(b_{15}\right)^{(1)} T_{14}-\left[\left(b_{15}^{\prime}\right)^{(1)}-\left(b_{15}^{\prime \prime}\right)^{(1)}(G, t)\right] T_{15} \\
& +\left(a_{13}^{\prime \prime}\right)^{(1)}\left(T_{14}, t\right)=\text { First augmentation factor } \\
& -\left(b_{13}^{\prime \prime}\right)^{(1)}(G, t)=\text { First detritions factor }
\end{aligned}
$$

\section{GOVERNING EQUATIONS:OF THE SYSTEM KANPSACK PROBLEM AND HAMILTONIAN PATH PROBLEM}

The differential system of this model is now

$$
\begin{aligned}
& \frac{d G_{16}}{d t}=\left(a_{16}\right)^{(2)} G_{17}-\left[\left(a_{16}^{\prime}\right)^{(2)}+\left(a_{16}^{\prime \prime}\right)^{(2)}\left(T_{17}, t\right)\right] G_{16} \\
& \frac{d G_{17}}{d t}=\left(a_{17}\right)^{(2)} G_{16}-\left[\left(a_{17}^{\prime}\right)^{(2)}+\left(a_{17}^{\prime \prime}\right)^{(2)}\left(T_{17}, t\right)\right] G_{17} \\
& \frac{d G_{18}}{d t}=\left(a_{18}\right)^{(2)} G_{17}-\left[\left(a_{18}^{\prime}\right)^{(2)}+\left(a_{18}^{\prime \prime}\right)^{(2)}\left(T_{17}, t\right)\right] G_{18} \\
& \frac{d T_{16}}{d t}=\left(b_{16}\right)^{(2)} T_{17}-\left[\left(b_{16}^{\prime}\right)^{(2)}-\left(b_{16}^{\prime \prime}\right)^{(2)}\left(\left(G_{19}\right), t\right)\right] T_{16}
\end{aligned}
$$


$\frac{d T_{17}}{d t}=\left(b_{17}\right)^{(2)} T_{16}-\left[\left(b_{17}^{\prime}\right)^{(2)}-\left(b_{17}^{\prime \prime}\right)^{(2)}\left(\left(G_{19}\right), t\right)\right] T_{17}$

$\frac{d T_{18}}{d t}=\left(b_{18}\right)^{(2)} T_{17}-\left[\left(b_{18}^{\prime}\right)^{(2)}-\left(b_{18}^{\prime \prime}\right)^{(2)}\left(\left(G_{19}\right), t\right)\right] T_{18}$

$+\left(a_{16}^{\prime \prime}\right)^{(2)}\left(T_{17}, t\right)=$ First augmentation factor

$-\left(b_{16}^{\prime \prime}\right)^{(2)}\left(\left(G_{19}\right), t\right)=$ First detritions factor

GOVERNING EQUATIONS: OF THE SYSTEM TRAVELLING SALESMAN PROBLEM AND SUBGRAPH ISOMERISM PROBLEM:

The differential system of this model is now

$\frac{d G_{20}}{d t}=\left(a_{20}\right)^{(3)} G_{21}-\left[\left(a_{20}^{\prime}\right)^{(3)}+\left(a_{20}^{\prime \prime}\right)^{(3)}\left(T_{21}, t\right)\right] G_{20}$

$\frac{d G_{21}}{d t}=\left(a_{21}\right)^{(3)} G_{20}-\left[\left(a_{21}^{\prime}\right)^{(3)}+\left(a_{21}^{\prime \prime}\right)^{(3)}\left(T_{21}, t\right)\right] G_{21}$

$\frac{d G_{22}}{d t}=\left(a_{22}\right)^{(3)} G_{21}-\left[\left(a_{22}^{\prime}\right)^{(3)}+\left(a_{22}^{\prime \prime}\right)^{(3)}\left(T_{21}, t\right)\right] G_{22}$

$\frac{d T_{20}}{d t}=\left(b_{20}\right)^{(3)} T_{21}-\left[\left(b_{20}^{\prime}\right)^{(3)}-\left(b_{20}^{\prime \prime}\right)^{(3)}\left(G_{23}, t\right)\right] T_{20}$

$\frac{d T_{21}}{d t}=\left(b_{21}\right)^{(3)} T_{20}-\left[\left(b_{21}^{\prime}\right)^{(3)}-\left(b_{21}^{\prime \prime}\right)^{(3)}\left(G_{23}, t\right)\right] T_{21}$

$\frac{d T_{22}}{d t}=\left(b_{22}\right)^{(3)} T_{21}-\left[\left(b_{22}^{\prime}\right)^{(3)}-\left(b_{22}^{\prime \prime}\right)^{(3)}\left(G_{23}, t\right)\right] T_{22}$

$+\left(a_{20}^{\prime \prime}\right)^{(3)}\left(T_{21}, t\right)=$ First augmentation factor

$-\left(b_{20}^{\prime \prime}\right)^{(3)}\left(G_{23}, t\right)=$ First detritions factor

\section{GOVERNING EQUATIONS:OF THE SYSTEM SUBSET SUM PROBLEM AND CLIQUE} PROBLEM

The differential system of this model is now

$$
\begin{aligned}
& \frac{d G_{24}}{d t}=\left(a_{24}\right)^{(4)} G_{25}-\left[\left(a_{24}^{\prime}\right)^{(4)}+\left(a_{24}^{\prime \prime}\right)^{(4)}\left(T_{25}, t\right)\right] G_{24} \\
& \frac{d G_{25}}{d t}=\left(a_{25}\right)^{(4)} G_{24}-\left[\left(a_{25}^{\prime}\right)^{(4)}+\left(a_{25}^{\prime \prime}\right)^{(4)}\left(T_{25}, t\right)\right] G_{25} \\
& \frac{d G_{26}}{d t}=\left(a_{26}\right)^{(4)} G_{25}-\left[\left(a_{26}^{\prime}\right)^{(4)}+\left(a_{26}^{\prime \prime}\right)^{(4)}\left(T_{25}, t\right)\right] G_{26} \\
& \frac{d T_{24}}{d t}=\left(b_{24}\right)^{(4)} T_{25}-\left[\left(b_{24}^{\prime}\right)^{(4)}-\left(b_{24}^{\prime \prime}\right)^{(4)}\left(\left(G_{27}\right), t\right)\right] T_{24} \\
& \frac{d T_{25}}{d t}=\left(b_{25}\right)^{(4)} T_{24}-\left[\left(b_{25}^{\prime}\right)^{(4)}-\left(b_{25}^{\prime \prime}\right)^{(4)}\left(\left(G_{27}\right), t\right)\right] T_{25} \\
& \frac{d T_{26}}{d t}=\left(b_{26}\right)^{(4)} T_{25}-\left[\left(b_{26}^{\prime}\right)^{(4)}-\left(b_{26}^{\prime \prime}\right)^{(4)}\left(\left(G_{27}\right), t\right)\right] T_{26} \\
& +\left(a_{24}^{\prime \prime}\right)^{(4)}\left(T_{25}, t\right)=\text { First augmentation factor } \\
& -\left(b_{24}^{\prime \prime}\right)^{(4)}\left(\left(G_{27}\right), t\right)=\text { First detritions factor }
\end{aligned}
$$




\section{GOVERNING EQUATIONS:OF THE SYSTEM VERTEX COVER PROBLEM AND INDEPENDENT SET PROBLEM}

The differential system of this model is now

$\frac{d G_{28}}{d t}=\left(a_{28}\right)^{(5)} G_{29}-\left[\left(a_{28}^{\prime}\right)^{(5)}+\left(a_{28}^{\prime \prime}\right)^{(5)}\left(T_{29}, t\right)\right] G_{28}$

$\frac{d G_{29}}{d t}=\left(a_{29}\right)^{(5)} G_{28}-\left[\left(a_{29}^{\prime}\right)^{(5)}+\left(a_{29}^{\prime \prime}\right)^{(5)}\left(T_{29}, t\right)\right] G_{29}$

$\frac{d G_{30}}{d t}=\left(a_{30}\right)^{(5)} G_{29}-\left[\left(a_{30}^{\prime}\right)^{(5)}+\left(a_{30}^{\prime \prime}\right)^{(5)}\left(T_{29}, t\right)\right] G_{30}$

$\frac{d T_{28}}{d t}=\left(b_{28}\right)^{(5)} T_{29}-\left[\left(b_{28}^{\prime}\right)^{(5)}-\left(b_{28}^{\prime \prime}\right)^{(5)}\left(\left(G_{31}\right), t\right)\right] T_{28}$

$\frac{d T_{29}}{d t}=\left(b_{29}\right)^{(5)} T_{28}-\left[\left(b_{29}^{\prime}\right)^{(5)}-\left(b_{29}^{\prime \prime}\right)^{(5)}\left(\left(G_{31}\right), t\right)\right] T_{29}$

$\frac{d T_{30}}{d t}=\left(b_{30}\right)^{(5)} T_{29}-\left[\left(b_{30}^{\prime}\right)^{(5)}-\left(b_{30}^{\prime \prime}\right)^{(5)}\left(\left(G_{31}\right), t\right)\right] T_{30}$

$+\left(a_{28}^{\prime \prime}\right)^{(5)}\left(T_{29}, t\right)=$ First augmentation factor

$-\left(b_{28}^{\prime \prime}\right)^{(5)}\left(\left(G_{31}\right), t\right)=$ First detritions factor

GOVERNING EQUATIONS:OF THE DOMINATING SET PROBLEM AND GRAPH COLORING PROBLEM:

The differential system of this model is now

$\frac{d G_{32}}{d t}=\left(a_{32}\right)^{(6)} G_{33}-\left[\left(a_{32}^{\prime}\right)^{(6)}+\left(a_{32}^{\prime \prime}\right)^{(6)}\left(T_{33}, t\right)\right] G_{32}$

$\frac{d G_{33}}{d t}=\left(a_{33}\right)^{(6)} G_{32}-\left[\left(a_{33}^{\prime}\right)^{(6)}+\left(a_{33}^{\prime \prime}\right)^{(6)}\left(T_{33}, t\right)\right] G_{33}$

$\frac{d G_{34}}{d t}=\left(a_{34}\right)^{(6)} G_{33}-\left[\left(a_{34}^{\prime}\right)^{(6)}+\left(a_{34}^{\prime \prime}\right)^{(6)}\left(T_{33}, t\right)\right] G_{34}$

$\frac{d T_{32}}{d t}=\left(b_{32}\right)^{(6)} T_{33}-\left[\left(b_{32}^{\prime}\right)^{(6)}-\left(b_{32}^{\prime \prime}\right)^{(6)}\left(\left(G_{35}\right), t\right)\right] T_{32}$

$\frac{d T_{33}}{d t}=\left(b_{33}\right)^{(6)} T_{32}-\left[\left(b_{33}^{\prime}\right)^{(6)}-\left(b_{33}^{\prime \prime}\right)^{(6)}\left(\left(G_{35}\right), t\right)\right] T_{33}$

$\frac{d T_{34}}{d t}=\left(b_{34}\right)^{(6)} T_{33}-\left[\left(b_{34}^{\prime}\right)^{(6)}-\left(b_{34}^{\prime \prime}\right)^{(6)}\left(\left(G_{35}\right), t\right)\right] T_{34}$

$+\left(a_{32}^{\prime \prime}\right)^{(6)}\left(T_{33}, t\right)=$ First augmentation factor

$-\left(b_{32}^{\prime \prime}\right)^{(6)}\left(\left(G_{35}\right), t\right)=$ First detritions factor

FINAL CONCATENATED GOVERNING EQUATIONS OF THE SYSTEM:

1. BOOLEAN SATISFIABILITY PROBLEM

2. N PUZZLE

3. KNAPSACK PROBLEM

4. HAMILTONIAN PATH PROBLEM

5. TRAVELLING SALESMAN PROBLEM

6. SUB GRAPH ISOMERISM PROBLEM 
7. SUBSET SUM PROBLEM

8. CLIQUE PROBLEM

9. VERTEX COVER PROBLEM

10. INDEPENDENT SET PROBLEM

11. DOMINATING SET PROBLEM

12. GRAPH COLORING PROBLEM

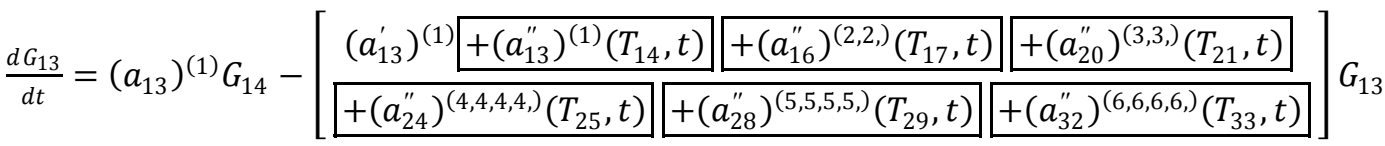

$\frac{d G_{14}}{d t}=\left(a_{14}\right)^{(1)} G_{13}-\left[\begin{array}{c|c|c|}\left(a_{14}^{\prime}\right)^{(1)}+\left(a_{14}^{\prime \prime}\right)^{(1)}\left(T_{14}, t\right) & +\left(a_{17}^{\prime \prime}\right)^{(2,2,)}\left(T_{17}, t\right) & +\left(a_{21}^{\prime \prime}\right)^{(3,3,)}\left(T_{21}, t\right) \\ \hdashline+\left(a_{25}^{\prime \prime}\right)^{(4,4,4,4,4)}\left(T_{25}, t\right) & +\left(a_{29}^{\prime \prime}\right)^{(5,5,5,5,)}\left(T_{29}, t\right) & +\left(a_{33}^{\prime \prime}\right)^{(6,6,6,6,}\left(T_{33}, t\right)\end{array}\right] G_{14}$

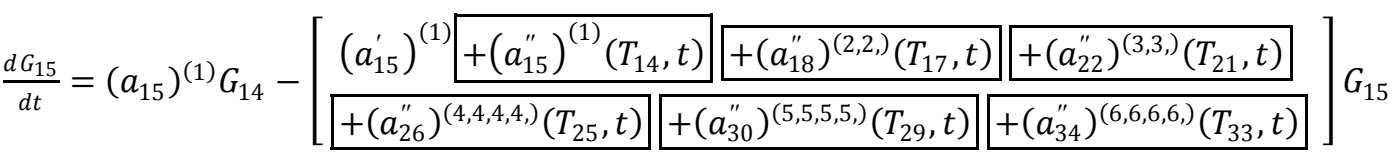

Where $\left(a_{13}^{\prime \prime}\right)^{(1)}\left(T_{14}, t\right),\left(a_{14}^{\prime \prime}\right)^{(1)}\left(T_{14}, t\right),\left(a_{15}^{\prime \prime}\right)^{(1)}\left(T_{14}, t\right)$ are first augmentation coefficients for category 1,2 and 3

$+\left(a_{16}^{\prime \prime}\right)^{(2,2,)}\left(T_{17}, t\right),+\left(a_{17}^{\prime \prime}\right)^{(2,2,)}\left(T_{17}, t\right),+\left(a_{18}^{\prime \prime}\right)^{(2,2,)}\left(T_{17}, t\right)$ are second augmentation coefficient for category 1,2 and 3

$+\left(a_{20}^{\prime \prime}\right)^{(3,3,)}\left(T_{21}, t\right),+\left(a_{21}^{\prime \prime}\right)^{(3,3,)}\left(T_{21}, t\right),+\left(a_{22}^{\prime \prime}\right)^{(3,3,)}\left(T_{21}, t\right)$ are third augmentation coefficient for category 1,2 and 3

$+\left(a_{24}^{\prime \prime}\right)^{(4,4,4,4,)}\left(T_{25}, t\right),+\left(a_{25}^{\prime \prime}\right)^{(4,4,4,4,)}\left(T_{25}, t\right),+\left(a_{26}^{\prime \prime}\right)^{(4,4,4,4,)}\left(T_{25}, t\right)$ are fourth augmentation coefficient for category 1,2 and 3

$+\left(a_{28}^{\prime \prime}\right)^{(5,5,5,5,)}\left(T_{29}, t\right),+\left(a_{29}^{\prime \prime}\right)^{(5,5,5,5,)}\left(T_{29}, t\right),+\left(a_{30}^{\prime \prime}\right)^{(5,5,5,5,)}\left(T_{29}, t\right)$ are fifth augmentation coefficient for category 1,2 and 3

$+\left(a_{32}^{\prime \prime}\right)^{(6,6,6,6,6)}\left(T_{33}, t\right),+\left(a_{33}^{\prime \prime}\right)^{(6,6,6,6,6)}\left(T_{33}, t\right),+\left(a_{34}^{\prime \prime}\right)^{(6,6,6,6,6)}\left(T_{33}, t\right)$ are sixth augmentation coefficient for category 1,2 and 3

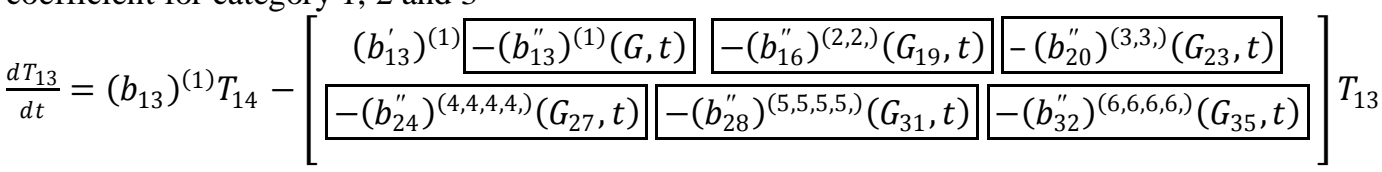

$\frac{d T_{14}}{d t}=\left(b_{14}\right)^{(1)} T_{13}-\left[\frac{\left(b_{14}^{\prime}\right)^{(1)}-\left(b_{14}^{\prime \prime}\right)^{(1)}(G, t)--\left(b_{17}^{\prime \prime}\right)^{(2,2,)}\left(G_{19}, t\right)}{-\left(b_{21}^{\prime \prime}\right)^{(3,3,)}\left(G_{23}, t\right)}\right]$

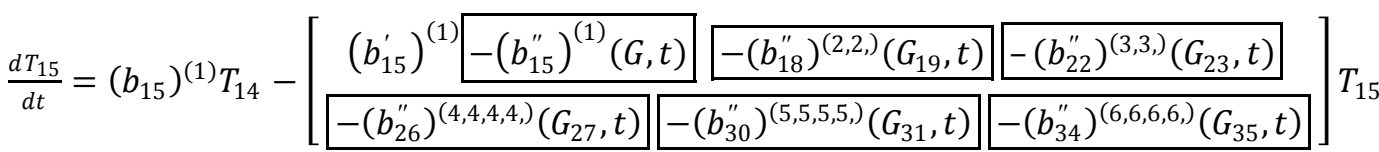

Where $-\left(b_{13}^{\prime \prime}\right)^{(1)}(G, t),-\left(b_{14}^{\prime \prime}\right)^{(1)}(G, t),-\left(b_{15}^{\prime \prime}\right)^{(1)}(G, t)$ are first detrition coefficients for category 63 1,2 and 3

$-\left(b_{16}^{\prime \prime}\right)^{(2,2,)}\left(G_{19}, t\right),-\left(b_{17}^{\prime \prime}\right)^{(2,2,)}\left(G_{19}, t\right),-\left(b_{18}^{\prime \prime}\right)^{(2,2,)}\left(G_{19}, t\right)$ are second detrition coefficients for category 1,2 and 3

$-\left(b_{20}^{\prime \prime}\right)^{(3,3,)}\left(G_{23}, t\right),-\left(b_{21}^{\prime \prime}\right)^{(3,3,)}\left(G_{23}, t\right),-\left(b_{22}^{\prime \prime}\right)^{(3,3,)}\left(G_{23}, t\right)$ are third detrition coefficients for category 1,2 and 3 
Internal Differentiation, Comparative Variability, Structural Morphology, Normative Aspect Of Prognostication Of Ipse Dixit Np Hard Problems-A Totalistic Paradigmatic Statement

$-\left(b_{24}^{\prime \prime}\right)^{(4,4,4,4,)}\left(G_{27}, t\right),-\left(b_{25}^{\prime \prime}\right)^{(4,4,4,4,4)}\left(G_{27}, t\right),-\left(b_{26}^{\prime \prime}\right)^{(4,4,4,4,)}\left(G_{27}, t\right)$ are fourth detrition coefficients for category 1,2 and 3

$-\left(b_{28}^{\prime \prime}\right)^{(5,5,5,5,)}\left(G_{31}, t\right),-\left(b_{29}^{\prime \prime}\right)^{(5,5,5,5,)}\left(G_{31}, t\right),-\left(b_{30}^{\prime \prime}\right)^{(5,5,5,5,)}\left(G_{31}, t\right)$ are fifth detrition coefficients for category 1,2 and 3

$-\left(b_{32}^{\prime \prime}\right)^{(6,6,6,6,)}\left(G_{35}, t\right),-\left(b_{33}^{\prime \prime}\right)^{(6,6,6,6,)}\left(G_{35}, t\right),-\left(b_{34}^{\prime \prime}\right)^{(6,6,6,6,6,)}\left(G_{35}, t\right)$ are sixth detrition coefficients for category 1,2 and 3

$\frac{d G_{16}}{d t}=\left(a_{16}\right)^{(2)} G_{17}-\left[\begin{array}{cc|c|}\left(a_{16}^{\prime}\right)^{(2)}+\left(a_{16}^{\prime \prime}\right)^{(2)}\left(T_{17}, t\right) & +\left(a_{13}^{\prime \prime}\right)^{(1,1,)}\left(T_{14}, t\right) & +\left(a_{20}^{\prime \prime}\right)^{(3,3,3)}\left(T_{21}, t\right) \\ \hline+\left(a_{24}^{\prime \prime}\right)^{(4,4,4,4,4)}\left(T_{25}, t\right) & +\left(a_{28}^{\prime \prime}\right)^{(5,5,5,5,5)}\left(T_{29}, t\right) & +\left(a_{32}^{\prime \prime}\right)^{(6,6,6,6,6)}\left(T_{33}, t\right)\end{array}\right] G_{16}$

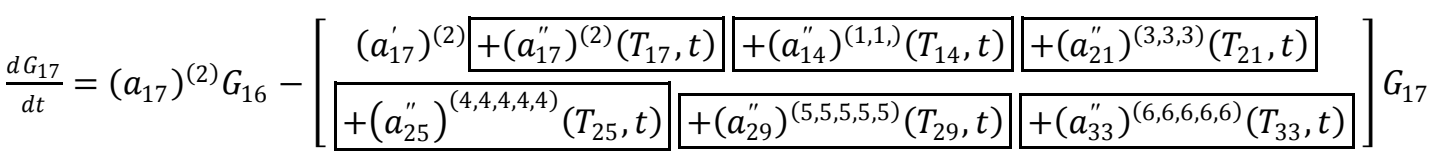

$\frac{d G_{18}}{d t}=\left(a_{18}\right)^{(2)} G_{17}-\left[\begin{array}{c}\left(a_{18}^{\prime}\right)^{(2)}+\left(a_{18}^{\prime \prime}\right)^{(2)}\left(T_{17}, t\right)+\left(a_{15}^{\prime \prime}\right)^{(1,1,)}\left(T_{14}, t\right)+\left(a_{22}^{\prime \prime}\right)^{(3,3,3)}\left(T_{21}, t\right) \\ ++\left(a_{26}^{\prime \prime}\right)^{(4,4,4,4,4)}\left(T_{25}, t\right)+\left(a_{30}^{\prime \prime}\right)^{(5,5,5,5,5)}\left(T_{29}, t\right) \\ ++\left(a_{34}^{\prime \prime}\right)^{(6,6,6,6,6,6}\left(T_{33}, t\right)\end{array}\right] G_{18}$

Where $+\left(a_{16}^{\prime \prime}\right)^{(2)}\left(T_{17}, t\right),+\left(a_{17}^{\prime \prime}\right)^{(2)}\left(T_{17}, t\right),+\left(a_{18}^{\prime \prime}\right)^{(2)}\left(T_{17}, t\right)$ are first augmentation coefficients for category 1,2 and 3

$+\left(a_{13}^{\prime \prime}\right)^{(1,1,)}\left(T_{14}, t\right),+\left(a_{14}^{\prime \prime}\right)^{(1,1,)}\left(T_{14}, t\right),+\left(a_{15}^{\prime \prime}\right)^{(1,1,)}\left(T_{14}, t\right)$ are second augmentation coefficient for category 1,2 and 3

$+\left(a_{20}^{\prime \prime}\right)^{(3,3,3)}\left(T_{21}, t\right),+\left(a_{21}^{\prime \prime}\right)^{(3,3,3)}\left(T_{21}, t\right),+\left(a_{22}^{\prime \prime}\right)^{(3,3,3)}\left(T_{21}, t\right)$ are third augmentation coefficient for category 1,2 and 3

$+\left(a_{24}^{\prime \prime}\right)^{(4,4,4,4,4)}\left(T_{25}, t\right),+\left(a_{25}^{\prime \prime}\right)^{(4,4,4,4,4)}\left(T_{25}, t\right),+\left(a_{26}^{\prime \prime}\right)^{(4,4,4,4,4)}\left(T_{25}, t\right)$ are fourth augmentation coefficient for category 1,2 and 3

$+\left(a_{28}^{\prime \prime}\right)^{(5,5,5,5,5)}\left(T_{29}, t\right),+\left(a_{29}^{\prime \prime}\right)^{(5,5,5,5,5)}\left(T_{29}, t\right),+\left(a_{30}^{\prime \prime}\right)^{(5,5,5,5,5)}\left(T_{29}, t\right)$ are fifth augmentation coefficient for category 1,2 and 3

$+\left(a_{32}^{\prime \prime}\right)^{(6,6,6,6,6)}\left(T_{33}, t\right),+\left(a_{33}^{\prime \prime}\right)^{(6,6,6,6,6)}\left(T_{33}, t\right),+\left(a_{34}^{\prime \prime}\right)^{(6,6,6,6,6)}\left(T_{33}, t\right)$ are sixth augmentation coefficient for category 1,2 and 3

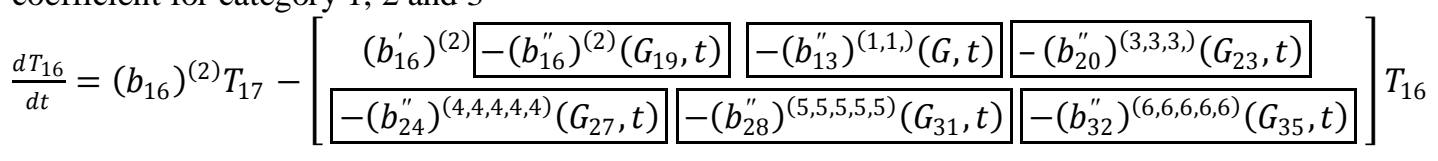

$\frac{d T_{17}}{d t}=\left(b_{17}\right)^{(2)} T_{16}-\left[\begin{array}{c}\left(b_{17}^{\prime}\right)^{(2)}-\left(b_{17}^{\prime \prime}\right)^{(2)}\left(G_{19}, t\right) \\ -\left(b_{25}^{\prime \prime}\right)^{(4,4,4,4,4)}\left(G_{27}, t\right)-\left(b_{14}^{\prime \prime}\right)^{(1,1,)}(G, t)-\left(b_{29}^{\prime \prime}\right)^{(5,5,5,5,5)}\left(G_{31}, t\right)-\left(b_{33}^{\prime \prime}\right)^{(6,6,3,3,3,)}\left(G_{23}, t\right) \\ \left.-G_{35}, t\right)\end{array}\right] T_{17}$

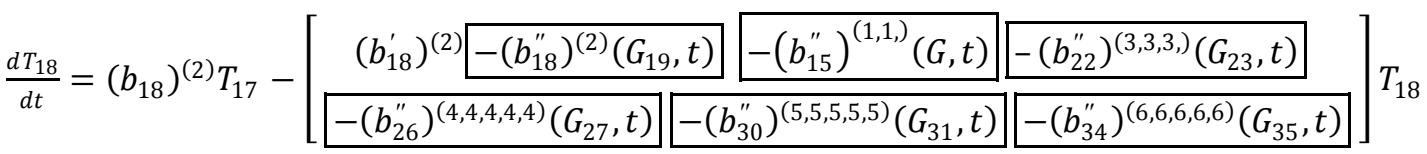

where $-\left(\mathrm{b}_{16}^{\prime \prime}\right)^{(2)}\left(\mathrm{G}_{19}, \mathrm{t}\right),-\left(\mathrm{b}_{17}^{\prime \prime}\right)^{(2)}\left(\mathrm{G}_{19}, \mathrm{t}\right),-\left(\mathrm{b}_{18}^{\prime \prime}\right)^{(2)}\left(\mathrm{G}_{19}, \mathrm{t}\right)$ are first detrition coefficients for category 1,2 and 3

$-\left(b_{13}^{\prime \prime}\right)^{(1,1,)}(G, t),-\left(b_{14}^{\prime \prime}\right)^{(1,1,)}(G, t),-\left(b_{15}^{\prime \prime}\right)^{(1,1,)}(G, t)$ are second detrition coefficients for category 1,2 and 3

$-\left(b_{20}^{\prime \prime}\right)^{(3,3,3,)}\left(G_{23}, t\right),-\left(b_{21}^{\prime \prime}\right)^{(3,3,3,)}\left(G_{23}, t\right),-\left(b_{22}^{\prime \prime}\right)^{(3,3,3,)}\left(G_{23}, t\right)$ are third detrition coefficients for category 1,2 and 3 
Internal Differentiation, Comparative Variability, Structural Morphology, Normative Aspect Of Prognostication Of Ipse Dixit Np Hard Problems-A Totalistic Paradigmatic Statement

$-\left(b_{24}^{\prime \prime}\right)^{(4,4,4,4,4)}\left(G_{27}, t\right),-\left(b_{25}^{\prime \prime}\right)^{(4,4,4,4,4)}\left(G_{27}, t\right)-\left(b_{26}^{\prime \prime}\right)^{(4,4,4,4,4)}\left(G_{27}, t\right)$ are fourth detrition

coefficients for category 1,2 and 3

$-\left(b_{28}^{\prime \prime}\right)^{(5,5,5,5,5)}\left(G_{31}, t\right),-\left(b_{29}^{\prime \prime}\right)^{(5,5,5,5,5)}\left(G_{31}, t\right),-\left(b_{30}^{\prime \prime}\right)^{(5,5,5,5,5)}\left(G_{31}, t\right)$ are fifth detrition coefficients for category 1,2 and 3

$-\left(b_{32}^{\prime \prime}\right)^{(6,6,6,6,6)}\left(G_{35}, t\right),-\left(b_{33}^{\prime \prime}\right)^{(6,6,6,6,6)}\left(G_{35}, t\right),-\left(b_{34}^{\prime \prime}\right)^{(6,6,6,6,6,6)}\left(G_{35}, t\right)$ are sixth detrition coefficients for category 1,2 and 3

$\frac{d G_{20}}{d t}=\left(a_{20}\right)^{(3)} G_{21}-\left[\begin{array}{c|c|c|}\left(a_{20}^{\prime}\right)^{(3)}+\left(a_{20}^{\prime \prime}\right)^{(3)}\left(T_{21}, t\right) & +\left(a_{16}^{\prime \prime}\right)^{(2,2,2)}\left(T_{17}, t\right) & +\left(a_{13}^{\prime \prime}\right)^{(1,1,1,1)}\left(T_{14}, t\right) \\ \hdashline+\left(a_{24}^{\prime \prime}\right)^{(4,4,4,4,4,4,4)}\left(T_{25}, t\right) & +\left(a_{28}^{\prime \prime}\right)^{(5,5,5,5,5,5)}\left(T_{29}, t\right) & +\left(a_{32}^{\prime \prime}\right)^{(6,6,6,6,6,6,6)}\left(T_{33}, t\right)\end{array}\right] G_{20}$

$\frac{d G_{21}}{d t}=\left(a_{21}\right)^{(3)} G_{20}-\left[\begin{array}{c|c|c|}\left(a_{21}^{\prime}\right)^{(3)}+\left(a_{21}^{\prime \prime}\right)^{(3)}\left(T_{21}, t\right) & +\left(a_{17}^{\prime \prime}\right)^{(2,2,2)}\left(T_{17}, t\right) & +\left(a_{14}^{\prime \prime}\right)^{(1,1,1,1)}\left(T_{14}, t\right) \\ \hdashline+\left(a_{25}^{\prime \prime}\right)^{(4,4,4,4,4,4,4)}\left(T_{25}, t\right) & +\left(a_{29}^{\prime \prime}\right)^{(5,5,5,5,5,5)}\left(T_{29}, t\right) & +\left(a_{33}^{\prime \prime}\right)^{(6,6,6,6,6,6}\left(T_{33}, t\right)\end{array}\right] G_{21}$

$\frac{d G_{22}}{d t}=\left(a_{22}\right)^{(3)} G_{21}-\left[\begin{array}{c|c|c|c|}\left(a_{22}^{\prime}\right)^{(3)}+\left(a_{22}^{\prime \prime}\right)^{(3)}\left(T_{21}, t\right) & +\left(a_{18}^{\prime \prime}\right)^{(2,2,2)}\left(T_{17}, t\right) & +\left(a_{15}^{\prime \prime}\right)^{(1,1,1,1)}\left(T_{14}, t\right) \\ \hdashline+\left(a_{26}^{\prime \prime}\right)^{(4,4,4,4,4,4)}\left(T_{25}, t\right) & +\left(a_{30}^{\prime \prime}\right)^{(5,5,5,5,5,5)}\left(T_{29}, t\right) & +\left(a_{34}^{\prime \prime}\right)^{(6,6,6,6,6,6)}\left(T_{33}, t\right)\end{array}\right] G_{22}$

$+\left(a_{20}^{\prime \prime}\right)^{(3)}\left(T_{21}, t\right),+\left(a_{21}^{\prime \prime}\right)^{(3)}\left(T_{21}, t\right),+\left(a_{22}^{\prime \prime}\right)^{(3)}\left(T_{21}, t\right)$ are first augmentation coefficients for category 1,2 and 3

$+\left(a_{16}^{\prime \prime}\right)^{(2,2,2)}\left(T_{17}, t\right),+\left(a_{17}^{\prime \prime}\right)^{(2,2,2)}\left(T_{17}, t\right),+\left(a_{18}^{\prime \prime}\right)^{(2,2,2)}\left(T_{17}, t\right)$ are second augmentation coefficients for category 1,2 and 3

$+\left(a_{13}^{\prime \prime}\right)^{(1,1,1,)}\left(T_{14}, t\right),+\left(a_{14}^{\prime \prime}\right)^{(1,1,1,)}\left(T_{14}, t\right),+\left(a_{15}^{\prime \prime}\right)^{(1,1,1,)}\left(T_{14}, t\right)$ are third augmentation coefficients for category 1,2 and 3

$+\left(a_{24}^{\prime \prime}\right)^{(4,4,4,4,4,4)}\left(T_{25}, t\right),+\left(a_{25}^{\prime \prime}\right)^{(4,4,4,4,4,4)}\left(T_{25}, t\right),+\left(a_{26}^{\prime \prime}\right)^{(4,4,4,4,4,4)}\left(T_{25}, t\right)$ are fourth augmentation coefficients for category 1,2 and 3

$+\left(a_{28}^{\prime \prime}\right)^{(5,5,5,5,5,5)}\left(T_{29}, t\right),+\left(a_{29}^{\prime \prime}\right)^{(5,5,5,5,5,5)}\left(T_{29}, t\right),+\left(a_{30}^{\prime \prime}\right)^{(5,5,5,5,5,5)}\left(T_{29}, t\right)$ are fifth augmentation coefficients for category 1,2 and 3

$+\left(a_{32}^{\prime \prime}\right)^{(6,6,6,6,6,6,6)}\left(T_{33}, t\right),+\left(a_{33}^{\prime \prime}\right)^{(6,6,6,6,6,6,6)}\left(T_{33}, t\right),+\left(a_{34}^{\prime \prime}\right)^{(6,6,6,6,6,6,6)}\left(T_{33}, t\right)$ are sixth augmentation coefficients for category 1,2 and 3

$\frac{d T_{20}}{d t}=\left(b_{20}\right)^{(3)} T_{21}-\left[\begin{array}{c|c|c|c|}\left(b_{20}^{\prime}\right)^{(3)}-\left(b_{20}^{\prime \prime}\right)^{(3)}\left(G_{23}, t\right) & -\left(b_{16}^{\prime \prime}\right)^{(2,2,2)}\left(G_{19}, t\right) & -\left(b_{13}^{\prime \prime}\right)^{(1,1,1,1)}(G, t) \\ \hline-\left(b_{24}^{\prime \prime}\right)^{(4,4,4,4,4,4)}\left(G_{27}, t\right) & -\left(b_{28}^{\prime \prime}\right)^{(5,5,5,5,5,5)}\left(G_{31}, t\right) & -\left(b_{32}^{\prime \prime}\right)^{(6,6,6,6,6,6,6)}\left(G_{35}, t\right)\end{array}\right] T_{20}$

$\frac{d T_{21}}{d t}=\left(b_{21}\right)^{(3)} T_{20}-\left[\begin{array}{c|c|c|}\left(b_{21}^{\prime}\right)^{(3)}-\left(b_{21}^{\prime \prime}\right)^{(3)}\left(G_{23}, t\right) & -\left(b_{17}^{\prime \prime}\right)^{(2,2,2)}\left(G_{19}, t\right) & -\left(b_{14}^{\prime \prime}\right)^{(1,1,1,1)}(G, t) \\ \hline-\left(b_{25}^{\prime \prime}\right)^{(4,4,4,4,4,4)}\left(G_{27}, t\right) & -\left(b_{29}^{\prime \prime}\right)^{(5,5,5,5,5,5)}\left(G_{31}, t\right) & -\left(b_{33}^{\prime \prime}\right)^{(6,6,6,6,6,6,6)}\left(G_{35}, t\right)\end{array}\right] T_{21}$

$\frac{d T_{22}}{d t}=\left(b_{22}\right)^{(3)} T_{21}-\left[\begin{array}{c|c|c|}\left(b_{22}^{\prime}\right)^{(3)}-\left(b_{22}^{\prime \prime}\right)^{(3)}\left(G_{23}, t\right) & -\left(b_{18}^{\prime \prime}\right)^{(2,2,2)}\left(G_{19}, t\right) & -\left(b_{15}^{\prime \prime}\right)^{(1,1,1,1)}(G, t) \\ \hline-\left(b_{26}^{\prime \prime}\right)^{(4,4,4,4,4,4)}\left(G_{27}, t\right) & -\left(b_{30}^{\prime \prime}\right)^{(5,5,5,5,5,5)}\left(G_{31}, t\right) & -\left(b_{34}^{\prime \prime}\right)^{(6,6,6,6,6,6)}\left(G_{35}, t\right)\end{array}\right] T_{22}$

$-\left(b_{20}^{\prime \prime}\right)^{(3)}\left(G_{23}, t\right),-\left(b_{21}^{\prime \prime}\right)^{(3)}\left(G_{23}, t\right),-\left(b_{22}^{\prime \prime}\right)^{(3)}\left(G_{23}, t\right)$ are first detrition coefficients for category 91 1,2 and 3

$-\left(b_{16}^{\prime \prime}\right)^{(2,2,2)}\left(G_{19}, t\right),-\left(b_{17}^{\prime \prime}\right)^{(2,2,2)}\left(G_{19}, t\right),-\left(b_{18}^{\prime \prime}\right)^{(2,2,2)}\left(G_{19}, t\right)$ are second detrition coefficients for category 1, 2 and 3 
Internal Differentiation, Comparative Variability, Structural Morphology, Normative Aspect Of Prognostication Of Ipse Dixit Np Hard Problems-A Totalistic Paradigmatic Statement

$-\left(b_{13}^{\prime \prime}\right)^{(1,1,1,)}(G, t),-\left(b_{14}^{\prime \prime}\right)^{(1,1,1,)}(G, t),-\left(b_{15}^{\prime \prime}\right)^{(1,1,1,)}(G, t)$ are third detrition coefficients for category 1,2 and 3 $-\left(b_{24}^{\prime \prime}\right)^{(4,4,4,4,4,4)}\left(G_{27}, t\right),-\left(b_{25}^{\prime \prime}\right)^{(4,4,4,4,4,4)}\left(G_{27}, t\right),-\left(b_{26}^{\prime \prime}\right)^{(4,4,4,4,4,4)}\left(G_{27}, t\right)$ are fourth detrition coefficients for category 1,2 and 3 $-\left(b_{28}^{\prime \prime}\right)^{(5,5,5,5,5,5)}\left(G_{31}, t\right),-\left(b_{29}^{\prime \prime}\right)^{(5,5,5,5,5,5)}\left(G_{31}, t\right),-\left(b_{30}^{\prime \prime}\right)^{(5,5,5,5,5,5)}\left(G_{31}, t\right)$ are fifth detrition coefficients for category 1,2 and 3 $-\left(b_{32}^{\prime \prime}\right)^{(6,6,6,6,6,6,6)}\left(G_{35}, t\right),-\left(b_{33}^{\prime \prime}\right)^{(6,6,6,6,6,6,6)}\left(G_{35}, t\right),-\left(b_{34}^{\prime \prime}\right)^{(6,6,6,6,6,6,6)}\left(G_{35}, t\right)$ are sixth detrition coefficients for category 1,2 and 3

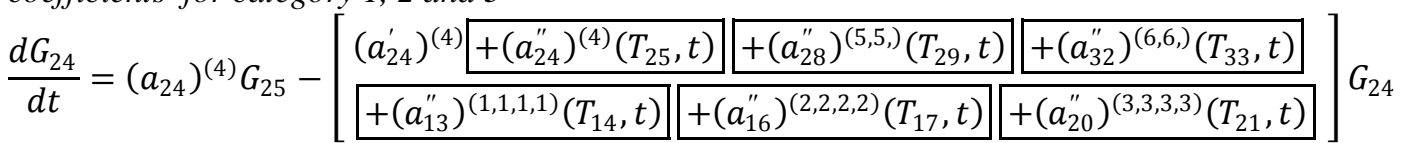

$\frac{d G_{25}}{d t}=\left(a_{25}\right)^{(4)} G_{24}-\left[\begin{array}{l}\left(a_{25}^{\prime}\right)^{(4)}+\left(a_{25}^{\prime \prime}\right)^{(4)}\left(T_{25}, t\right)+\left(a_{29}^{\prime \prime}\right)^{(5,5,)}\left(T_{29}, t\right)+\left(a_{33}^{\prime \prime}\right)^{(6,6)}\left(T_{33}, t\right) \\ ++\left(a_{14}^{\prime \prime}\right)^{(1,1,1,1)}\left(T_{14}, t\right)+\left(a_{17}^{\prime \prime}\right)^{(2,2,2,2)}\left(T_{17}, t\right)+\left(a_{21}^{\prime \prime}\right)^{(3,3,3,3)}\left(T_{21}, t\right)\end{array}\right] G_{25}$

$\frac{d G_{26}}{d t}=\left(a_{26}\right)^{(4)} G_{25}-\left[\begin{array}{l}\left(a_{26}^{\prime}\right)^{(4)}+\left(a_{26}^{\prime \prime}\right)^{(4)}\left(T_{25}, t\right)+\left(a_{30}^{\prime \prime}\right)^{(5,5,)}\left(T_{29}, t\right)+\left(a_{34}^{\prime \prime}\right)^{(6,6,)}\left(T_{33}, t\right) \\ +\left(a_{15}^{\prime \prime}\right)^{(1,1,1,1)}\left(T_{14}, t\right)+\left(a_{18}^{\prime \prime}\right)^{(2,2,2,2)}\left(T_{17}, t\right)+\left(a_{22}^{\prime \prime}\right)^{(3,3,3,3)}\left(T_{21}, t\right)\end{array}\right] G_{26}$

Where $\left(a_{24}^{\prime \prime}\right)^{(4)}\left(T_{25}, t\right),\left(a_{25}^{\prime \prime}\right)^{(4)}\left(T_{25}, t\right),\left(a_{26}^{\prime \prime}\right)^{(4)}\left(T_{25}, t\right)$ are first augmentation coefficients for cate gory 1,2 and 3 $+\left(a_{28}^{\prime \prime}\right)^{(5,5,)}\left(T_{29}, t\right),+\left(a_{29}^{\prime \prime}\right)^{(5,5,)}\left(T_{29}, t\right),+\left(a_{30}^{\prime \prime}\right)^{(5,5,)}\left(T_{29}, t\right)$ are second augmentation coefficient for category 1,2 and 3 $+\left(a_{32}^{\prime \prime}\right)^{(6,6)}\left(T_{33}, t\right),+\left(a_{33}^{\prime \prime}\right)^{(6,6,)}\left(T_{33}, t\right),+\left(a_{34}^{\prime \prime}\right)^{(6,6,)}\left(T_{33}, t\right)$ are third augmentation coefficient for category 1,2 and 3 $+\left(a_{13}^{\prime \prime}\right)^{(1,1,1,1)}\left(T_{14}, t\right),+\left(a_{14}^{\prime \prime}\right)^{(1,1,1,1)}\left(T_{14}, t\right),+\left(a_{15}^{\prime \prime}\right)^{(1,1,1,1)}\left(T_{14}, t\right)$ are fourth augmentation coefficients for category 1,2 ,and 3 $+\left(a_{16}^{\prime \prime}\right)^{(2,2,2,2)}\left(T_{17}, t\right),++\left(a_{17}^{\prime \prime}\right)^{(2,2,2,2)}\left(T_{17}, t\right),++\left(a_{18}^{\prime \prime}\right)^{(2,2,2,2)}\left(T_{17}, t\right)$ are fifth augmentation coefficients for category 1,2 ,and 3 $+\left(a_{20}^{\prime \prime}\right)^{(3,3,3,3)}\left(T_{21}, t\right),+\left(a_{21}^{\prime \prime}\right)^{(3,3,3,3)}\left(T_{21}, t\right),+\left(a_{22}^{\prime \prime}\right)^{(3,3,3,3)}\left(T_{21}, t\right)$ are sixth augmentation coefficients for category 1,2 ,and 3

$\frac{d T_{24}}{d t}=\left(b_{24}\right)^{(4)} T_{25}-\left[\begin{array}{c}\left(b_{24}^{\prime}\right)^{(4)}-\left(b_{24}^{\prime \prime}\right)^{(4)}\left(G_{27}, t\right)-\left(b_{28}^{\prime \prime}\right)^{(5,5,)}\left(G_{31}, t\right)-\left(b_{32}^{\prime \prime}\right)^{(6,6,)}\left(G_{35}, t\right) \\ -\left(b_{13}^{\prime \prime}\right)^{(1,1,1,1)}(G, t)-\left(b_{16}^{\prime \prime}\right)^{(2,2,2,2)}\left(G_{19}, t\right)-\left(b_{20}^{\prime \prime}\right)^{(3,3,3,3)}\left(G_{23}, t\right)\end{array}\right] T_{24}$

$\frac{d T_{25}}{d t}=\left(b_{25}\right)^{(4)} T_{24}-\left[\begin{array}{c}\left(b_{25}^{\prime}\right)^{(4)}-\left(b_{25}^{\prime \prime}\right)^{(4)}\left(G_{27}, t\right)-\left(b_{29}^{\prime \prime}\right)^{(5,5,)}\left(G_{31}, t\right)-\left(b_{33}^{\prime \prime}\right)^{(6,6,)}\left(G_{35}, t\right) \\ -\left(b_{14}^{\prime \prime}\right)^{(1,1,1,1)}(G, t)-\left(b_{17}^{\prime \prime}\right)^{(2,2,2,2)}\left(G_{19}, t\right)-\left(b_{21}^{\prime \prime}\right)^{(3,3,3,3)}\left(G_{23}, t\right)\end{array}\right] T_{25}$

$\frac{d T_{26}}{d t}=\left(b_{26}\right)^{(4)} T_{25}-\left[\begin{array}{c}\left(b_{26}^{\prime}\right)^{(4)}-\left(b_{26}^{\prime \prime}\right)^{(4)}\left(G_{27}, t\right)-\left(b_{30}^{\prime \prime}\right)^{(5,5,)}\left(G_{31}, t\right)-\left(b_{34}^{\prime \prime}\right)^{(6,6,)}\left(G_{35}, t\right) \\ -\left(b_{15}^{\prime \prime}\right)^{(1,1,1,1)}(G, t)-\left(b_{18}^{\prime \prime}\right)^{(2,2,2,2)}\left(G_{19}, t\right)-\left(b_{22}^{\prime \prime}\right)^{(3,3,3,3)}\left(G_{23}, t\right)\end{array}\right] T_{26}$

Where $-\left(b_{24}^{\prime \prime}\right)^{(4)}\left(G_{27}, t\right),-\left(b_{25}^{\prime \prime}\right)^{(4)}\left(G_{27}, t\right),-\left(b_{26}^{\prime \prime}\right)^{(4)}\left(G_{27}, t\right)$ are first detrition coefficients for category 1,2 and 3 $-\left(b_{28}^{\prime \prime}\right)^{(5,5,)}\left(G_{31}, t\right),-\left(b_{29}^{\prime \prime}\right)^{(5,5,)}\left(G_{31}, t\right),-\left(b_{30}^{\prime \prime}\right)^{(5,5,)}\left(G_{31}, t\right)$ are second detrition coefficients for category 1,2 and 3 
Internal Differentiation, Comparative Variability, Structural Morphology, Normative Aspect Of Prognostication Of Ipse Dixit Np Hard Problems-A Totalistic Paradigmatic Statement

$-\left(b_{32}^{\prime \prime}\right)^{(6,6,)}\left(G_{35}, t\right),-\left(b_{33}^{\prime \prime}\right)^{(6,6,)}\left(G_{35}, t\right),-\left(b_{34}^{\prime \prime}\right)^{(6,6,)}\left(G_{35}, t\right)$ are third detrition

coefficients for category 1,2 and 3

$-\left(b_{13}^{\prime \prime}\right)^{(1,1,1,1)}(G, t),-\left(b_{14}^{\prime \prime}\right)^{(1,1,1,1)}(G, t),-\left(b_{15}^{\prime \prime}\right)^{(1,1,1,1)}(G, t)$

are fourth detrition coef ficients for category 1,2 and 3

$-\left(b_{16}^{\prime \prime}\right)^{(2,2,2,2)}\left(G_{19}, t\right),-\left(b_{17}^{\prime \prime}\right)^{(2,2,2,2)}\left(G_{19}, t\right),-\left(b_{18}^{\prime \prime}\right)^{(2,2,2,2)}\left(G_{19}, t\right)$

are fif th detrition coef ficients for cate gory 1,2 and 3

$-\left(b_{20}^{\prime \prime}\right)^{(3,3,3,3)}\left(G_{23}, t\right),-\left(b_{21}^{\prime \prime}\right)^{(3,3,3,3)}\left(G_{23}, t\right),-\left(b_{22}^{\prime \prime}\right)^{(3,3,3,3)}\left(G_{23}, t\right)$

are sixth detrition coef ficients for category 1,2 and 3

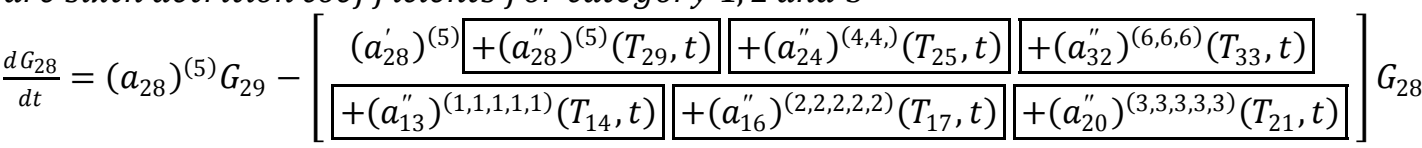

$\frac{d G_{29}}{d t}=\left(a_{29}\right)^{(5)} G_{28}-\left[\begin{array}{c|c|c|c|}\left(a_{29}^{\prime}\right)^{(5)}+\left(a_{29}^{\prime \prime}\right)^{(5)}\left(T_{29}, t\right) & +\left(a_{25}^{\prime \prime}\right)^{(4,4,)}\left(T_{25}, t\right) & +\left(a_{33}^{\prime \prime}\right)^{(6,6,6)}\left(T_{33}, t\right) \\ ++\left(a_{14}^{\prime \prime}\right)^{(1,1,1,1,1)}\left(T_{14}, t\right) & +\left(a_{17}^{\prime \prime}\right)^{(2,2,2,2,2)}\left(T_{17}, t\right) & +\left(a_{21}^{\prime \prime}\right)^{(3,3,3,3,3)}\left(T_{21}, t\right)\end{array}\right] G_{29}$

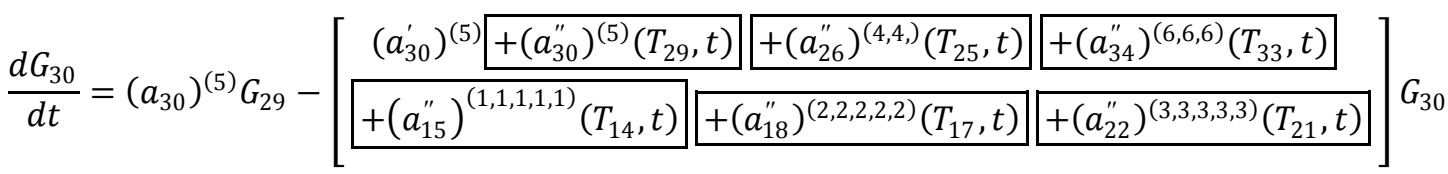

Where $+\left(a_{28}^{\prime \prime}\right)^{(5)}\left(T_{29}, t\right),+\left(a_{29}^{\prime \prime}\right)^{(5)}\left(T_{29}, t\right),+\left(a_{30}^{\prime \prime}\right)^{(5)}\left(T_{29}, t\right)$ are first augmentation coefficients for category 1,2 and 3

And $+\left(a_{24}^{\prime \prime}\right)^{(4,4,)}\left(T_{25}, t\right),+\left(a_{25}^{\prime \prime}\right)^{(4,4,)}\left(T_{25}, t\right),+\left(a_{26}^{\prime \prime}\right)^{(4,4,)}\left(T_{25}, t\right)$ are second augmentation coefficient for category 1,2 and 3

$+\left(a_{32}^{\prime \prime}\right)^{(6,6,6)}\left(T_{33}, t\right),+\left(a_{33}^{\prime \prime}\right)^{(6,6,6)}\left(T_{33}, t\right),+\left(a_{34}^{\prime \prime}\right)^{(6,6,6)}\left(T_{33}, t\right)$ are third augmentation coefficient for category 1,2 and 3

$+\left(a_{13}^{\prime \prime}\right)^{(1,1,1,1,1)}\left(T_{14}, t\right),+\left(a_{14}^{\prime \prime}\right)^{(1,1,1,1,1)}\left(T_{14}, t\right),+\left(a_{15}^{\prime \prime}\right)^{(1,1,1,1,1)}\left(T_{14}, t\right)$ are fourth augmentation coefficients for category 1,2 , and 3

$+\left(a_{16}^{\prime \prime}\right)^{(2,2,2,2,2)}\left(T_{17}, t\right),+\left(a_{17}^{\prime \prime}\right)^{(2,2,2,2,2)}\left(T_{17}, t\right),+\left(a_{18}^{\prime \prime}\right)^{(2,2,2,2,2)}\left(T_{17}, t\right)$ are fifth augmentation coefficients for category 1,2 ,and 3

$+\left(a_{20}^{\prime \prime}\right)^{(3,3,3,3,3)}\left(T_{21}, t\right),+\left(a_{21}^{\prime \prime}\right)^{(3,3,3,3,3)}\left(T_{21}, t\right),+\left(a_{22}^{\prime \prime}\right)^{(3,3,3,3,3)}\left(T_{21}, t\right)$ are sixth augmentation coefficients for category $1,2,3$

$\frac{d T_{28}}{d t}=\left(b_{28}\right)^{(5)} T_{29}-\left[\begin{array}{c}\left(b_{28}^{\prime}\right)^{(5)}-\left(b_{28}^{\prime \prime}\right)^{(5)}\left(G_{31}, t\right)-\left(b_{24}^{\prime \prime}\right)^{(4,4,)}\left(G_{27}, t\right)-\left(b_{32}^{\prime \prime}\right)^{(6,6,6)}\left(G_{35}, t\right) \\ --\left(b_{13}^{\prime \prime}\right)^{(1,1,1,1,1)}(G, t)--\left(b_{16}^{\prime \prime}\right)^{(2,2,2,2,2)}\left(G_{19}, t\right)-\left(b_{20}^{\prime \prime}\right)^{(3,3,3,3,3)}\left(G_{23}, t\right)\end{array}\right] T_{28}$

$\frac{d T_{29}}{d t}=\left(b_{29}\right)^{(5)} T_{28}-\left[\begin{array}{l}\left(b_{29}^{\prime}\right)^{(5)}-\left(b_{29}^{\prime \prime}\right)^{(5)}\left(G_{31}, t\right)-\left(b_{25}^{\prime \prime}\right)^{(4,4,)}\left(G_{27}, t\right)-\left(b_{33}^{\prime \prime}\right)^{(6,6,6)}\left(G_{35}, t\right) \\ -\left(b_{14}^{\prime \prime}\right)^{(1,1,1,1,1)}(G, t)--\left(b_{17}^{\prime \prime}\right)^{(2,2,2,2,2)}\left(G_{19}, t\right)-\left(b_{21}^{\prime \prime}\right)^{(3,3,3,3,3)}\left(G_{23}, t\right)\end{array}\right] T_{29}$

$\frac{d T_{30}}{d t}=\left(b_{30}\right)^{(5)} T_{29}-\left[\begin{array}{c}\left(b_{30}^{\prime}\right)^{(5)}-\left(b_{30}^{\prime \prime}\right)^{(5)}\left(G_{31}, t\right)-\left(b_{26}^{\prime \prime}\right)^{(4,4,)}\left(G_{27}, t\right)-\left(b_{34}^{\prime \prime}\right)^{(6,6,6)}\left(G_{35}, t\right) \\ -\left(b_{15}^{\prime \prime}\right)^{(1,1,1,1,1,)}(G, t)-\left(b_{18}^{\prime \prime}\right)^{(2,2,2,2,2)}\left(G_{19}, t\right)-\left(b_{22}^{\prime \prime}\right)^{(3,3,3,3,3)}\left(G_{23}, t\right)\end{array}\right] T_{30}$

where $-\left(b_{28}^{\prime \prime}\right)^{(5)}\left(G_{31}, t\right),-\left(b_{29}^{\prime \prime}\right)^{(5)}\left(G_{31}, t\right),-\left(b_{30}^{\prime \prime}\right)^{(5)}\left(G_{31}, t\right)$

are first detrition coef ficients for category 1,2 and 3 
Internal Differentiation, Comparative Variability, Structural Morphology, Normative Aspect Of Prognostication Of Ipse Dixit Np Hard Problems-A Totalistic Paradigmatic Statement

$-\left(b_{24}^{\prime \prime}\right)^{(4,4,)}\left(G_{27}, t\right),-\left(b_{25}^{\prime \prime}\right)^{(4,4,)}\left(G_{27}, t\right),-\left(b_{26}^{\prime \prime}\right)^{(4,4,)}\left(G_{27}, t\right)$ are second detrition

coefficients for category 1,2 and 3

$-\left(b_{32}^{\prime \prime}\right)^{(6,6,6)}\left(G_{35}, t\right),-\left(b_{33}^{\prime \prime}\right)^{(6,6,6)}\left(G_{35}, t\right),-\left(b_{34}^{\prime \prime}\right)^{(6,6,6)}\left(G_{35}, t\right)$ are third detrition

coefficients for category 1,2 and 3

$-\left(b_{13}^{\prime \prime}\right)^{(1,1,1,1,1)}(G, t),-\left(b_{14}^{\prime \prime}\right)^{(1,1,1,1,1)}(G, t),-\left(b_{15}^{\prime \prime}\right)^{(1,1,1,1,1,)}(G, t)$ are fourth detrition coefficients

for category 1,2 , and 3

$-\left(b_{16}^{\prime \prime}\right)^{(2,2,2,2,2)}\left(G_{19}, t\right),-\left(b_{17}^{\prime \prime}\right)^{(2,2,2,2,2)}\left(G_{19}, t\right),-\left(b_{18}^{\prime \prime}\right)^{(2,2,2,2,2)}\left(G_{19}, t\right)$ are fifth detrition coefficients for category 1,2 , and 3

$-\left(b_{20}^{\prime \prime}\right)^{(3,3,3,3,3)}\left(G_{23}, t\right),-\left(b_{21}^{\prime \prime}\right)^{(3,3,3,3,3)}\left(G_{23}, t\right),-\left(b_{22}^{\prime \prime}\right)^{(3,3,3,3,3)}\left(G_{23}, t\right)$ are sixth detrition coefficients for category 1,2 , and 3

$\frac{d G_{32}}{d t}=\left(a_{32}\right)^{(6)} G_{33}-\left[\begin{array}{c|c|c|}\left(a_{32}^{\prime}\right)^{(6)}+\left(a_{32}^{\prime \prime}\right)^{(6)}\left(T_{33}, t\right) & +\left(a_{28}^{\prime \prime}\right)^{(5,5,5)}\left(T_{29}, t\right) & +\left(a_{24}^{\prime \prime}\right)^{(4,4,4,}\left(T_{25}, t\right) \\ \hdashline+\left(a_{13}^{\prime \prime}\right)^{(1,1,1,1,1,1,1)}\left(T_{14}, t\right) & +\left(a_{16}^{\prime \prime}\right)^{(2,2,2,2,2,2)}\left(T_{17}, t\right) & +\left(a_{20}^{\prime \prime}\right)^{(3,3,3,3,3,3,3)}\left(T_{21}, t\right)\end{array}\right] G_{32}$

$\frac{d G_{33}}{d t}=\left(a_{33}\right)^{(6)} G_{32}-\left[\begin{array}{c|c|c|}\left(a_{33}^{\prime}\right)^{(6)}+\left(a_{33}^{\prime \prime}\right)^{(6)}\left(T_{33}, t\right) & +\left(a_{29}^{\prime \prime}\right)^{(5,5,5)}\left(T_{29}, t\right) & +\left(a_{25}^{\prime \prime}\right)^{(4,4,4,)}\left(T_{25}, t\right) \\ \hdashline+\left(a_{14}^{\prime \prime}\right)^{(1,1,1,1,1,1,1)}\left(T_{14}, t\right) & +\left(a_{17}^{\prime \prime}\right)^{(2,2,2,2,2,2)}\left(T_{17}, t\right) & +\left(a_{21}^{\prime \prime}\right)^{(3,3,3,3,3,3)}\left(T_{21}, t\right)\end{array}\right] G_{33}$

$\frac{d G_{34}}{d t}=\left(a_{34}\right)^{(6)} G_{33}-\left[\begin{array}{c|c|c|c|}\left(a_{34}^{\prime}\right)^{(6)}+\left(a_{34}^{\prime \prime}\right)^{(6)}\left(T_{33}, t\right) & +\left(a_{30}^{\prime \prime}\right)^{(5,5,5)}\left(T_{29}, t\right) & +\left(a_{26}^{\prime \prime}\right)^{(4,4,4,)}\left(T_{25}, t\right) \\ \hdashline+\left(a_{15}^{\prime \prime}\right)^{(1,1,1,1,1,1,1)}\left(T_{14}, t\right) & +\left(a_{18}^{\prime \prime}\right)^{(2,2,2,2,2,2)}\left(T_{17}, t\right) & +\left(a_{22}^{\prime \prime}\right)^{(3,3,3,3,3,3)}\left(T_{21}, t\right)\end{array}\right] G_{34}$

$+\left(a_{32}^{\prime \prime}\right)^{(6)}\left(T_{33}, t\right),+\left(a_{33}^{\prime \prime}\right)^{(6)}\left(T_{33}, t\right),+\left(a_{34}^{\prime \prime}\right)^{(6)}\left(T_{33}, t\right)$ are first augmentation coefficients for category 1,2 and 3 $+\left(a_{28}^{\prime \prime}\right)^{(5,5,5)}\left(T_{29}, t\right),+\left(a_{29}^{\prime \prime}\right)^{(5,5,5)}\left(T_{29}, t\right),+\left(a_{30}^{\prime \prime}\right)^{(5,5,5)}\left(T_{29}, t\right)$ are second augmentation coefficients for category 1,2 and 3

$+\left(a_{24}^{\prime \prime}\right)^{(4,4,4,)}\left(T_{25}, t\right),+\left(a_{25}^{\prime \prime}\right)^{(4,4,4,)}\left(T_{25}, t\right),+\left(a_{26}^{\prime \prime}\right)^{(4,4,4,4)}\left(T_{25}, t\right)$ are third augmentation coefficients for category 1,2 and 3

$+\left(a_{13}^{\prime \prime}\right)^{(1,1,1,1,1,1)}\left(T_{14}, t\right),+\left(a_{14}^{\prime \prime}\right)^{(1,1,1,1,1,1)}\left(T_{14}, t\right),+\left(a_{15}^{\prime \prime}\right)^{(1,1,1,1,1,1)}\left(T_{14}, t\right)$ - are fourth augmentation coefficients

$+\left(a_{16}^{\prime \prime}\right)^{(2,2,2,2,2,2)}\left(T_{17}, t\right),+\left(a_{17}^{\prime \prime}\right)^{(2,2,2,2,2,2)}\left(T_{17}, t\right),+\left(a_{18}^{\prime \prime}\right)^{(2,2,2,2,2,2)}\left(T_{17}, t\right)$ - fifth augmentation coefficients

$+\left(a_{20}^{\prime \prime}\right)^{(3,3,3,3,3,3)}\left(T_{21}, t\right),+\left(a_{21}^{\prime \prime}\right)^{(3,3,3,3,3,3)}\left(T_{21}, t\right),+\left(a_{22}^{\prime \prime}\right)^{(3,3,3,3,3,3)}\left(T_{21}, t\right)$ sixth augmentation coefficients

$\frac{d T_{32}}{d t}=\left(b_{32}\right)^{(6)} T_{33}-\left[\begin{array}{cc|c|}\left(b_{32}^{\prime}\right)^{(6)}-\left(b_{32}^{\prime \prime}\right)^{(6)}\left(G_{35}, t\right) & -\left(b_{28}^{\prime \prime}\right)^{(5,5,5)}\left(G_{31}, t\right) & -\left(b_{24}^{\prime \prime}\right)^{(4,4,4,)}\left(G_{27}, t\right) \\ \hline-\left(b_{13}^{\prime \prime}\right)^{(1,1,1,1,1,1)}(G, t) & -\left(b_{16}^{\prime \prime}\right)^{(2,2,2,2,2,2)}\left(G_{19}, t\right) & -\left(b_{20}^{\prime \prime}\right)^{(3,3,3,3,3,3)}\left(G_{23}, t\right)\end{array}\right] T_{32}$

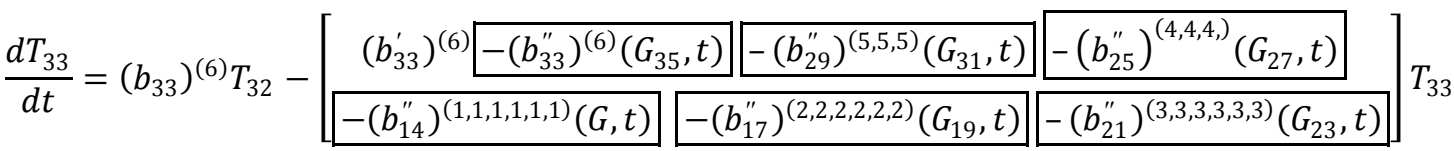

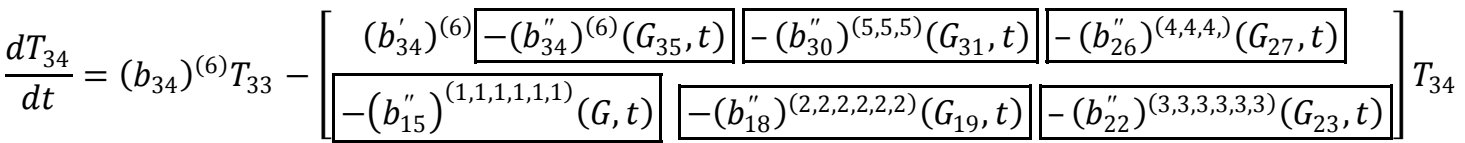

$-\left(b_{32}^{\prime \prime}\right)^{(6)}\left(G_{35}, t\right),-\left(b_{33}^{\prime \prime}\right)^{(6)}\left(G_{35}, t\right),-\left(b_{34}^{\prime \prime}\right)^{(6)}\left(G_{35}, t\right)$ are first detrition coefficients for category 1,2 and 3 
$-\left(b_{28}^{\prime \prime}\right)^{(5,5,5)}\left(G_{31}, t\right),-\left(b_{29}^{\prime \prime}\right)^{(5,5,5)}\left(G_{31}, t\right),-\left(b_{30}^{\prime \prime}\right)^{(5,5,5)}\left(G_{31}, t\right)$ are second detrition coefficients for category 1,2 and 3

$-\left(b_{24}^{\prime \prime}\right)^{(4,4,4,)}\left(G_{27}, t\right),-\left(b_{25}^{\prime \prime}\right)^{(4,4,4,)}\left(G_{27}, t\right),-\left(b_{26}^{\prime \prime}\right)^{(4,4,4,)}\left(G_{27}, t\right)$ are third detrition

coefficients for category 1,2 and 3

$-\left(b_{13}^{\prime \prime}\right)^{(1,1,1,1,1,1)}(G, t),-\left(b_{14}^{\prime \prime}\right)^{(1,1,1,1,1,1)}(G, t),-\left(b_{15}^{\prime \prime}\right)^{(1,1,1,1,1,1)}(G, t)$ are fourth detrition

coefficients for category 1,2 , and 3

$-\left(b_{16}^{\prime \prime}\right)^{(2,2,2,2,2,2)}\left(G_{19}, t\right),-\left(b_{17}^{\prime \prime}\right)^{(2,2,2,2,2,2)}\left(G_{19}, t\right),-\left(b_{18}^{\prime \prime}\right)^{(2,2,2,2,2,2)}\left(G_{19}, t\right)$ are fifth detrition coefficients for category 1,2 , and 3

$-\left(b_{20}^{\prime \prime}\right)^{(3,3,3,3,3,3)}\left(G_{23}, t\right),-\left(b_{21}^{\prime \prime}\right)^{(3,3,3,3,3,3)}\left(G_{23}, t\right),-\left(b_{22}^{\prime \prime}\right)^{(3,3,3,3,3,3)}\left(G_{23}, t\right)$ are sixth detrition coefficients for category 1,2 , and 3

Where we suppose

$$
\begin{aligned}
& \left(a_{i}\right)^{(1)},\left(a_{i}^{\prime}\right)^{(1)},\left(a_{i}^{\prime \prime}\right)^{(1)},\left(b_{i}\right)^{(1)},\left(b_{i}^{\prime}\right)^{(1)},\left(b_{i}^{\prime \prime}\right)^{(1)}>0, \\
& i, j=13,14,15
\end{aligned}
$$

(B) The functions $\left(a_{i}^{\prime \prime}\right)^{(1)},\left(b_{i}^{\prime \prime}\right)^{(1)}$ are positive continuous increasing and bounded.

Definition of $\left(p_{i}\right)^{(1)},\left(r_{i}\right)^{(1)}$ :

$$
\left(a_{i}^{\prime \prime}\right)^{(1)}\left(T_{14}, t\right) \leq\left(p_{i}\right)^{(1)} \leq\left(\hat{A}_{13}\right)^{(1)} 124
$$

$$
\left(b_{i}^{\prime \prime}\right)^{(1)}(G, t) \leq\left(r_{i}\right)^{(1)} \leq\left(b_{i}^{\prime}\right)^{(1)} \leq\left(\hat{B}_{13}\right)^{(1)}
$$

(C) $\quad \lim _{T_{2} \rightarrow \infty}\left(a_{i}^{\prime \prime}\right)^{(1)}\left(T_{14}, t\right)=\left(p_{i}\right)^{(1)}$

$$
\lim _{\mathrm{G} \rightarrow \infty}\left(b_{i}^{\prime \prime}\right)^{(1)}(G, t)=\left(r_{i}\right)^{(1)}
$$

Definition of $\left(\hat{A}_{13}\right)^{(1)},\left(\hat{B}_{13}\right)^{(1)}$ :

Where $\left(\hat{A}_{13}\right)^{(1)},\left(\hat{B}_{13}\right)^{(1)},\left(p_{i}\right)^{(1)},\left(r_{i}\right)^{(1)}$ are positive constants and $i=13,14,15$

They satisfy Lipschitz condition:

$$
\left|\left(a_{i}^{\prime \prime}\right)^{(1)}\left(T_{14}^{\prime}, t\right)-\left(a_{i}^{\prime \prime}\right)^{(1)}\left(T_{14}, t\right)\right| \leq\left(\hat{k}_{13}\right)^{(1)}\left|T_{14}-T_{14}^{\prime}\right| e^{-\left(M_{13}\right)^{(1)} t}
$$

$\left|\left(b_{i}^{\prime \prime}\right)^{(1)}\left(G^{\prime}, t\right)-\left(b_{i}^{\prime \prime}\right)^{(1)}(G, T)\right|<\left(\hat{k}_{13}\right)^{(1)} \| G-G^{\prime}|| e^{-\left(M_{13}\right)^{(1)} t}$

With the Lipschitz condition, we place a restriction on the behavior of functions $\left(a_{i}^{\prime \prime}\right)^{(1)}\left(T_{14}^{\prime}, t\right)$ and $\left(a_{i}^{\prime \prime}\right)^{(1)}\left(T_{14}, t\right) .\left(T_{14}^{\prime}, t\right)$ and $\left(T_{14}, t\right)$ are points belonging to the interval $\left[\left(\widehat{k}_{13}\right)^{(1)},\left(\widehat{M}_{13}\right)^{(1)}\right]$. It is to be noted that $\left(a_{i}^{\prime \prime}\right)^{(1)}\left(T_{14}, t\right)$ is uniformly continuous. In the eventuality of the fact, that if $\left(\widehat{M}_{13}\right)^{(1)}=1$ then the function $\left(a_{i}^{\prime \prime}\right)^{(1)}\left(T_{14}, t\right)$, the first augmentation coefficient would be absolutely continuous.

Definition of $\left(\widehat{M}_{13}\right)^{(1)},\left(\hat{k}_{13}\right)^{(1)}$ :

(D) $\quad\left(\widehat{M}_{13}\right)^{(1)},\left(\hat{k}_{13}\right)^{(1)}$, are positive constants

$$
\frac{\left(a_{i}\right)^{(1)}}{\left(\widetilde{M}_{13}\right)^{(1)}}, \frac{\left(b_{i}\right)^{(1)}}{\left(\widetilde{M}_{13}\right)^{(1)}}<1
$$

Definition of $\left(\hat{P}_{13}\right)^{(1)},\left(\hat{Q}_{13}\right)^{(1)}$ : 
(E) There exists two constants $\left(\hat{P}_{13}\right)^{(1)}$ and $\left(\hat{Q}_{13}\right)^{(1)}$ which together with $\left(\widehat{M}_{13}\right)^{(1)},\left(\hat{k}_{13}\right)^{(1)},\left(\hat{A}_{13}\right)^{(1)}$ and $\left(\hat{B}_{13}\right)^{(1)}$ and the constants $\left(a_{i}\right)^{(1)},\left(a_{i}^{\prime}\right)^{(1)},\left(b_{i}\right)^{(1)},\left(b_{i}^{\prime}\right)^{(1)},\left(p_{i}\right)^{(1)},\left(r_{i}\right)^{(1)}, i=13,14,15$,

satisfy the inequalities

$$
\begin{aligned}
& \frac{1}{\left(M_{13}\right)^{(1)}}\left[\left(a_{i}\right)^{(1)}+\left(a_{i}^{\prime}\right)^{(1)}+\left(\hat{A}_{13}\right)^{(1)}+\left(\hat{P}_{13}\right)^{(1)}\left(\hat{k}_{13}\right)^{(1)}\right]<1 \\
& \frac{1}{\left(\hat{M}_{13}\right)^{(1)}}\left[\left(b_{i}\right)^{(1)}+\left(b_{i}^{\prime}\right)^{(1)}+\left(\hat{B}_{13}\right)^{(1)}+\left(\hat{Q}_{13}\right)^{(1)}\left(\hat{k}_{13}\right)^{(1)}\right]<1
\end{aligned}
$$

Where we suppose

(F) $\quad\left(a_{i}\right)^{(2)},\left(a_{i}^{\prime}\right)^{(2)},\left(a_{i}^{\prime \prime}\right)^{(2)},\left(b_{i}\right)^{(2)},\left(b_{i}^{\prime}\right)^{(2)},\left(b_{i}^{\prime \prime}\right)^{(2)}>0, \quad i, j=16,17,18$

(G) The functions $\left(a_{i}^{\prime \prime}\right)^{(2)},\left(b_{i}^{\prime \prime}\right)^{(2)}$ are positive continuous increasing and bounded.

Definition of $\left(\mathrm{p}_{\mathrm{i}}\right)^{(2)},\left(\mathrm{r}_{\mathrm{i}}\right)^{(2)}$ :

$$
\left(a_{i}^{\prime \prime}\right)^{(2)}\left(T_{17}, t\right) \leq\left(p_{i}\right)^{(2)} \leq\left(\hat{A}_{16}\right)^{(2)}
$$

$\left(b_{i}^{\prime \prime}\right)^{(2)}\left(G_{19}, t\right) \leq\left(r_{i}\right)^{(2)} \leq\left(b_{i}^{\prime}\right)^{(2)} \leq\left(\hat{B}_{16}\right)^{(2)}$

(H)

$$
\begin{aligned}
& \lim _{T_{2} \rightarrow \infty}\left(a_{i}^{\prime \prime}\right)^{(2)}\left(T_{17}, t\right)=\left(p_{i}\right)^{(2)} \\
& \lim _{G \rightarrow \infty}\left(b_{i}^{\prime \prime}\right)^{(2)}\left(\left(G_{19}\right), t\right)=\left(r_{i}\right)^{(2)}
\end{aligned}
$$

Definition of $\left(\hat{A}_{16}\right)^{(2)},\left(\hat{B}_{16}\right)^{(2)}$ :

Where $\left(\hat{A}_{16}\right)^{(2)},\left(\hat{B}_{16}\right)^{(2)},\left(p_{i}\right)^{(2)},\left(r_{i}\right)^{(2)}$ are positive constants and $i=16,17,18$

They satisfy Lipschitz condition:

$$
\begin{aligned}
& \left|\left(a_{i}^{\prime \prime}\right)^{(2)}\left(T_{17}^{\prime}, t\right)-\left(a_{i}^{\prime \prime}\right)^{(2)}\left(T_{17}, t\right)\right| \leq\left(\hat{k}_{16}\right)^{(2)}\left|T_{17}-T_{17}^{\prime}\right| e^{-\left(M_{16}\right)^{(2)} t} \\
& \left|\left(b_{i}^{\prime \prime}\right)^{(2)}\left(\left(G_{19}\right)^{\prime}, t\right)-\left(b_{i}^{\prime \prime}\right)^{(2)}\left(\left(G_{19}\right), T_{19}\right)\right|<\left(\hat{k}_{16}\right)^{(2)}||\left(G_{19}\right)-\left(G_{19}\right)^{\prime}|| e^{-\left(M_{16}\right)^{(2)} t}
\end{aligned}
$$

With the Lipschitz condition, we place a restriction on the behavior of functions $\left(a_{i}^{\prime \prime}\right)^{(2)}\left(T_{17}^{\prime}, t\right)$ and $\left(a_{i}^{\prime \prime}\right)^{(2)}\left(T_{17}, t\right) .\left(T_{17}^{\prime}, t\right)$ And $\left(T_{17}, t\right)$ are points belonging to the interval $\left[\left(\hat{k}_{16}\right)^{(2)},\left(\widehat{M}_{16}\right)^{(2)}\right]$. It is to be noted that $\left(a_{i}^{\prime \prime}\right)^{(2)}\left(T_{17}, t\right)$ is uniformly continuous. In the eventuality of the fact, that if $\left(\widehat{M}_{16}\right)^{(2)}=1$ then the function $\left(a_{i}^{\prime \prime}\right)^{(2)}\left(T_{17}, t\right)$, the SECOND augmentation coefficient would be absolutely continuous.

Definition of $\left(\widehat{M}_{16}\right)^{(2)},\left(\hat{k}_{16}\right)^{(2)}$ :

(I) $\quad\left(\widehat{M}_{16}\right)^{(2)},\left(\hat{k}_{16}\right)^{(2)}$, are positive constants

$$
\frac{\left(a_{i}\right)^{(2)}}{\left(M_{16}\right)^{(2)}}, \frac{\left(b_{i}\right)^{(2)}}{\left(M_{16}\right)^{(2)}}<1
$$

Definition of $\left(\hat{P}_{13}\right)^{(2)},\left(\hat{Q}_{13}\right)^{(2)}$ :

There exists two constants $\left(\hat{P}_{16}\right)^{(2)}$ and $\left(\hat{Q}_{16}\right)^{(2)}$ which together with $\left(\widehat{M}_{16}\right)^{(2)},\left(\hat{k}_{16}\right)^{(2)},\left(\hat{A}_{16}\right)^{(2)}$ and $\left(\hat{B}_{16}\right)^{(2)}$ and the constants $\left(a_{i}\right)^{(2)},\left(a_{i}^{\prime}\right)^{(2)},\left(b_{i}\right)^{(2)},\left(b_{i}^{\prime}\right)^{(2)},\left(p_{i}\right)^{(2)},\left(r_{i}\right)^{(2)}, i=16,17,18$, 
satisfy the inequalities

$\frac{1}{\left(\widehat{\mathrm{M}}_{16}\right)^{(2)}}\left[\left(\mathrm{a}_{\mathrm{i}}\right)^{(2)}+\left(\mathrm{a}_{\mathrm{i}}^{\prime}\right)^{(2)}+\left(\widehat{\mathrm{A}}_{16}\right)^{(2)}+\left(\widehat{\mathrm{P}}_{16}\right)^{(2)}\left(\widehat{\mathrm{K}}_{16}\right)^{(2)}\right]<1$

$\frac{1}{\left(M_{16}\right)^{(2)}}\left[\left(b_{i}\right)^{(2)}+\left(b_{i}^{\prime}\right)^{(2)}+\left(\hat{B}_{16}\right)^{(2)}+\left(\hat{Q}_{16}\right)^{(2)}\left(\hat{k}_{16}\right)^{(2)}\right]<1$

Where we suppose

$$
\left(a_{i}\right)^{(3)},\left(a_{i}^{\prime}\right)^{(3)},\left(a_{i}^{\prime \prime}\right)^{(3)},\left(b_{i}\right)^{(3)},\left(b_{i}^{\prime}\right)^{(3)},\left(b_{i}^{\prime \prime}\right)^{(3)}>0, \quad i, j=20,21,22
$$

The functions $\left(a_{i}^{\prime \prime}\right)^{(3)},\left(b_{i}^{\prime \prime}\right)^{(3)}$ are positive continuous increasing and bounded.

Definition of $\left(p_{i}\right)^{(3)},\left(\mathrm{r}_{\mathrm{i}}\right)^{(3)}$ :

$$
\begin{gathered}
\left(a_{i}^{\prime \prime}\right)^{(3)}\left(T_{21}, t\right) \leq\left(p_{i}\right)^{(3)} \leq\left(\hat{A}_{20}\right)^{(3)} \\
\left(b_{i}^{\prime \prime}\right)^{(3)}(G, t) \leq\left(r_{i}\right)^{(3)} \leq\left(b_{i}^{\prime}\right)^{(3)} \leq\left(\hat{B}_{20}\right)^{(3)} \\
\lim _{T_{2} \rightarrow \infty}\left(a_{i}^{\prime \prime}\right)^{(3)}\left(T_{21}, t\right)=\left(p_{i}\right)^{(3)} \\
\lim _{\mathrm{G} \rightarrow \infty}\left(b_{i}^{\prime \prime}\right)^{(3)}(G, t)=\left(r_{i}\right)^{(3)}
\end{gathered}
$$

Definition of $\left(\hat{A}_{20}\right)^{(3)},\left(\hat{B}_{20}\right)^{(3)}$ :

Where $\left(\hat{A}_{20}\right)^{(3)},\left(\hat{B}_{20}\right)^{(3)},\left(p_{i}\right)^{(3)},\left(r_{i}\right)^{(3)}$ are positive constants and $i=20,21,22$

They satisfy Lipschitz condition:

$$
\begin{aligned}
& \left|\left(a_{i}^{\prime \prime}\right)^{(3)}\left(T_{21}^{\prime}, t\right)-\left(a_{i}^{\prime \prime}\right)^{(3)}\left(T_{21}, t\right)\right| \leq\left(\hat{k}_{20}\right)^{(3)}\left|T_{21}-T_{21}^{\prime}\right| e^{-\left(\hat{M}_{20}\right)^{(3)} t} \\
& \left|\left(b_{i}^{\prime \prime}\right)^{(3)}\left(G^{\prime}, t\right)-\left(b_{i}^{\prime \prime}\right)^{(3)}(G, T)\right|<\left(\hat{k}_{20}\right)^{(3)}|| G-G^{\prime}|| e^{-\left(\hat{M}_{20}\right)^{(3)} t}
\end{aligned}
$$

With the Lipschitz condition, we place a restriction on the behavior of functions $\left(a_{i}^{\prime \prime}\right)^{(3)}\left(T_{21}^{\prime}, t\right)$ and $\left(a_{i}^{\prime \prime}\right)^{(3)}\left(T_{21}, t\right) .\left(T_{21}^{\prime}, t\right)$ And $\left(T_{21}, t\right)$ are points belonging to the interval $\left[\left(\hat{k}_{20}\right)^{(3)},\left(\widehat{M}_{20}\right)^{(3)}\right]$. It is to be noted that $\left(a_{i}^{\prime \prime}\right)^{(3)}\left(T_{21}, t\right)$ is uniformly continuous. In the eventuality of the fact, that if $\left(\widehat{M}_{20}\right)^{(3)}=$ 1 then the function $\left(a_{i}^{\prime \prime}\right)^{(3)}\left(T_{21}, t\right)$, the third augmentation coefficient would be absolutely continuous.

Definition of $\left(\widehat{M}_{20}\right)^{(3)},\left(\hat{k}_{20}\right)^{(3)}$ :

$$
\begin{aligned}
& \left(\widehat{M}_{20}\right)^{(3)},\left(\hat{k}_{20}\right)^{(3)}, \text { are positive constants } \\
& \frac{\left(a_{i}\right)^{(3)}}{\left(M_{20}\right)^{(3)}}, \frac{\left(b_{i}\right)^{(3)}}{\left(\widehat{M}_{20}\right)^{(3)}}<1
\end{aligned}
$$

There exists two constants There exists two constants $\left(\hat{P}_{20}\right)^{(3)}$ and $\left(\hat{Q}_{20}\right)^{(3)}$ which together with $\left(\widehat{M}_{20}\right)^{(3)},\left(\hat{k}_{20}\right)^{(3)},\left(\hat{A}_{20}\right)^{(3)}$ and $\left(\hat{B}_{20}\right)^{(3)}$ and the constants $\left(a_{i}\right)^{(3)},\left(a_{i}^{\prime}\right)^{(3)},\left(b_{i}\right)^{(3)},\left(b_{i}^{\prime}\right)^{(3)},\left(p_{i}\right)^{(3)},\left(r_{i}\right)^{(3)}, i=20,21,22$, satisfy the inequalities

$$
\begin{aligned}
& \frac{1}{\left(M_{20}\right)^{(3)}}\left[\left(a_{i}\right)^{(3)}+\left(a_{i}^{\prime}\right)^{(3)}+\left(\hat{A}_{20}\right)^{(3)}+\left(\hat{P}_{20}\right)^{(3)}\left(\hat{k}_{20}\right)^{(3)}\right]<1 \\
& \frac{1}{\left(\widehat{M}_{20}\right)^{(3)}}\left[\left(b_{i}\right)^{(3)}+\left(b_{i}^{\prime}\right)^{(3)}+\left(\hat{B}_{20}\right)^{(3)}+\left(\hat{Q}_{20}\right)^{(3)}\left(\hat{k}_{20}\right)^{(3)}\right]<1
\end{aligned}
$$

Where we suppose 
Internal Differentiation, Comparative Variability, Structural Morphology, Normative Aspect Of Prognostication Of Ipse Dixit Np Hard Problems-A Totalistic Paradigmatic Statement

(L) $\quad\left(a_{i}\right)^{(4)},\left(a_{i}^{\prime}\right)^{(4)},\left(a_{i}^{\prime \prime}\right)^{(4)},\left(b_{i}\right)^{(4)},\left(b_{i}^{\prime}\right)^{(4)},\left(b_{i}^{\prime \prime}\right)^{(4)}>0, \quad i, j=24,25,26$

(M) The functions $\left(a_{i}^{\prime \prime}\right)^{(4)},\left(b_{i}^{\prime \prime}\right)^{(4)}$ are positive continuous increasing and bounded.

Definition of $\left(p_{i}\right)^{(4)},\left(r_{i}\right)^{(4)}$ :

$$
\begin{aligned}
& \left(a_{i}^{\prime \prime}\right)^{(4)}\left(T_{25}, t\right) \leq\left(p_{i}\right)^{(4)} \leq\left(\hat{A}_{24}\right)^{(4)} \\
& \left(b_{i}^{\prime \prime}\right)^{(4)}\left(\left(G_{27}\right), t\right) \leq\left(r_{i}\right)^{(4)} \leq\left(b_{i}^{\prime}\right)^{(4)} \leq\left(\hat{B}_{24}\right)^{(4)}
\end{aligned}
$$

$(N)$

$$
\begin{aligned}
& \lim _{T_{2} \rightarrow \infty}\left(a_{i}^{\prime \prime}\right)^{(4)}\left(T_{25}, t\right)=\left(p_{i}\right)^{(4)} \\
& \lim _{\mathrm{G} \rightarrow \infty}\left(b_{i}^{\prime \prime}\right)^{(4)}\left(\left(G_{27}\right), t\right)=\left(r_{i}\right)^{(4)}
\end{aligned}
$$

Definition of $\left(\hat{A}_{24}\right)^{(4)},\left(\hat{B}_{24}\right)^{(4)}$ :

Where $\left(\hat{A}_{24}\right)^{(4)},\left(\hat{B}_{24}\right)^{(4)},\left(p_{i}\right)^{(4)},\left(r_{i}\right)^{(4)}$ are positive constants and $i=24,25,26$

They satisfy Lipschitz condition:

$\left|\left(a_{i}^{\prime \prime}\right)^{(4)}\left(T_{25}^{\prime}, t\right)-\left(a_{i}^{\prime \prime}\right)^{(4)}\left(T_{25}, t\right)\right| \leq\left(\hat{k}_{24}\right)^{(4)}\left|T_{25}-T_{25}^{\prime}\right| e^{-\left(\widehat{M}_{24}\right)^{(4)} t}$

$\left|\left(b_{i}^{\prime \prime}\right)^{(4)}\left(\left(G_{27}\right)^{\prime}, t\right)-\left(b_{i}^{\prime \prime}\right)^{(4)}\left(\left(G_{27}\right), T\right)\right|<\left(\hat{k}_{24}\right)^{(4)}\left\|\left(G_{27}\right)-\left(G_{27}\right)^{\prime}\right\| e^{-\left(M_{24}\right)^{(4)} t}$

With the Lipschitz condition, we place a restriction on the behavior of functions $\left(a_{i}^{\prime \prime}\right)^{(4)}\left(T_{25}^{\prime}, t\right)$ and $\left(a_{i}^{\prime \prime}\right)^{(4)}\left(T_{25}, t\right) .\left(T_{25}^{\prime}, t\right)$ and $\left(T_{25}, t\right)$ are points belonging to the interval $\left[\left(\hat{k}_{24}\right)^{(4)},\left(\widehat{M}_{24}\right)^{(4)}\right]$. It is to be noted that $\left(a_{i}^{\prime \prime}\right)^{(4)}\left(T_{25}, t\right)$ is uniformly continuous. In the eventuality of the fact, that if $\left(\widehat{M}_{24}\right)^{(4)}=$ 4 then the function $\left(a_{i}^{\prime \prime}\right)^{(4)}\left(T_{25}, t\right)$, the fourth augmentation coefficient would be absolutely continuous.

Definition of $\left(\widehat{M}_{24}\right)^{(4)},\left(\hat{k}_{24}\right)^{(4)}$ :

(O) $\quad\left(\widehat{M}_{24}\right)^{(4)},\left(\hat{k}_{24}\right)^{(4)}$, are positive constants

$$
\frac{\left(a_{i}\right)^{(4)}}{\left(\widehat{M}_{24}\right)^{(4)}}, \frac{\left(b_{i}\right)^{(4)}}{\left(\widehat{M}_{24}\right)^{(4)}}<1
$$

Definition of $\left(\hat{P}_{24}\right)^{(4)},\left(\widehat{Q}_{24}\right)^{(4)}$ :

(P) There exists two constants $\left(\hat{P}_{24}\right)^{(4)}$ and $\left(\hat{Q}_{24}\right)^{(4)}$ which together with $\left(\widehat{M}_{24}\right)^{(4)},\left(\hat{k}_{24}\right)^{(4)},\left(\hat{A}_{24}\right)^{(4)}$ and $\left(\hat{B}_{24}\right)^{(4)}$ and the constants $\left(a_{i}\right)^{(4)},\left(a_{i}^{\prime}\right)^{(4)},\left(b_{i}\right)^{(4)},\left(b_{i}^{\prime}\right)^{(4)},\left(p_{i}\right)^{(4)},\left(r_{i}\right)^{(4)}, i=24,25,26$, satisfy the inequalities

$\frac{1}{\left(\widehat{M}_{24}\right)^{(4)}}\left[\left(a_{i}\right)^{(4)}+\left(a_{i}^{\prime}\right)^{(4)}+\left(\hat{A}_{24}\right)^{(4)}+\left(\hat{P}_{24}\right)^{(4)}\left(\hat{k}_{24}\right)^{(4)}\right]<1$

$\frac{1}{\left(M_{24}\right)^{(4)}}\left[\left(b_{i}\right)^{(4)}+\left(b_{i}^{\prime}\right)^{(4)}+\left(\hat{B}_{24}\right)^{(4)}+\left(\hat{Q}_{24}\right)^{(4)}\left(\hat{k}_{24}\right)^{(4)}\right]<1$

Where we suppose 
(Q) $\quad\left(a_{i}\right)^{(5)},\left(a_{i}^{\prime}\right)^{(5)},\left(a_{i}^{\prime \prime}\right)^{(5)},\left(b_{i}\right)^{(5)},\left(b_{i}^{\prime}\right)^{(5)},\left(b_{i}^{\prime \prime}\right)^{(5)}>0, \quad i, j=28,29,30$

(R) The functions $\left(a_{i}^{\prime \prime}\right)^{(5)},\left(b_{i}^{\prime \prime}\right)^{(5)}$ are positive continuous increasing and bounded.

Definition of $\left(p_{i}\right)^{(5)},\left(r_{i}\right)^{(5)}$ :

$$
\begin{aligned}
& \left(a_{i}^{\prime \prime}\right)^{(5)}\left(T_{29}, t\right) \leq\left(p_{i}\right)^{(5)} \leq\left(\hat{A}_{28}\right)^{(5)} \\
& \left(b_{i}^{\prime \prime}\right)^{(5)}\left(\left(G_{31}\right), t\right) \leq\left(r_{i}\right)^{(5)} \leq\left(b_{i}^{\prime}\right)^{(5)} \leq\left(\hat{B}_{28}\right)^{(5)}
\end{aligned}
$$

$$
\begin{gathered}
\lim _{T_{2} \rightarrow \infty}\left(a_{i}^{\prime \prime}\right)^{(5)}\left(T_{29}, t\right)=\left(p_{i}\right)^{(5)} \\
\lim _{G \rightarrow \infty}\left(b_{i}^{\prime \prime}\right)^{(5)}\left(G_{31}, t\right)=\left(r_{i}\right)^{(5)}
\end{gathered}
$$

Definition of $\left(\hat{A}_{28}\right)^{(5)},\left(\hat{B}_{28}\right)^{(5)}$ :

Where $\left(\hat{A}_{28}\right)^{(5)},\left(\hat{B}_{28}\right)^{(5)},\left(p_{i}\right)^{(5)},\left(r_{i}\right)^{(5)}$ are positive constants and $i=28,29,30$

They satisfy Lipschitz condition:

$$
\begin{aligned}
& \left|\left(a_{i}^{\prime \prime}\right)^{(5)}\left(T_{29}^{\prime}, t\right)-\left(a_{i}^{\prime \prime}\right)^{(5)}\left(T_{29}, t\right)\right| \leq\left(\hat{k}_{28}\right)^{(5)}\left|T_{29}-T_{29}^{\prime}\right| e^{-\left(M_{28}\right)^{(5)} t} \\
& \left|\left(b_{i}^{\prime \prime}\right)^{(5)}\left(\left(G_{31}\right)^{\prime}, t\right)-\left(b_{i}^{\prime \prime}\right)^{(5)}\left(\left(G_{31}\right),\left(T_{31}\right)\right)\right|<\left(\hat{k}_{28}\right)^{(5)}||\left(G_{31}\right)-\left(G_{31}\right)^{\prime}|| e^{-\left(M_{28}\right)^{(5)} t}
\end{aligned}
$$

With the Lipschitz condition, we place a restriction on the behavior of functions $\left(a_{i}^{\prime \prime}\right)^{(5)}\left(T_{29}^{\prime}, t\right)$ and $\left(a_{i}^{\prime \prime}\right)^{(5)}\left(T_{29}, t\right) .\left(T_{29}^{\prime}, t\right)$ And $\left(T_{29}, t\right)$ are points belonging to the interval $\left[\left(\hat{k}_{28}\right)^{(5)},\left(\widehat{M}_{28}\right)^{(5)}\right]$. It is to be noted that $\left(a_{i}^{\prime \prime}\right)^{(5)}\left(T_{29}, t\right)$ is uniformly continuous. In the eventuality of the fact, that if $\left(\widehat{M}_{28}\right)^{(5)}=$ 5 then the function $\left(a_{i}^{\prime \prime}\right)^{(5)}\left(T_{29}, t\right)$, the fifth augmentation coefficient would be absolutely continuous.

Definition of $\left(\widehat{M}_{28}\right)^{(5)},\left(\hat{k}_{28}\right)^{(5)}$ :

$$
\begin{aligned}
& \text { (T) } \quad\left(\widehat{M}_{28}\right)^{(5)},\left(\hat{k}_{28}\right)^{(5)} \text {, are positive } 184 \text { constants } \\
& \frac{\left(a_{i}\right)^{(5)}}{\left(M_{28}\right)^{(5)}}, \frac{\left(b_{i}\right)^{(5)}}{\left(M_{28}\right)^{(5)}}<1
\end{aligned}
$$

Definition of $\left(\hat{P}_{28}\right)^{(5)},\left(\hat{Q}_{28}\right)^{(5)}$ :

(U) There exists two constants $\left(\hat{P}_{28}\right)^{(5)}$ and $\left(\hat{Q}_{28}\right)^{(5)}$ which together with $\left(\widehat{M}_{28}\right)^{(5)},\left(\hat{k}_{28}\right)^{(5)},\left(\hat{A}_{28}\right)^{(5)}$ and $\left(\hat{B}_{28}\right)^{(5)}$ and the constants $\left(a_{i}\right)^{(5)},\left(a_{i}^{\prime}\right)^{(5)},\left(b_{i}\right)^{(5)},\left(b_{i}^{\prime}\right)^{(5)},\left(p_{i}\right)^{(5)},\left(r_{i}\right)^{(5)}, i=28,29,30, \quad$ satisfy the inequalities

$\frac{1}{\left(\widehat{M}_{28}\right)^{(5)}}\left[\left(a_{i}\right)^{(5)}+\left(a_{i}^{\prime}\right)^{(5)}+\left(\hat{A}_{28}\right)^{(5)}+\left(\hat{P}_{28}\right)^{(5)}\left(\hat{k}_{28}\right)^{(5)}\right]<1$

$\frac{1}{\left(\widehat{M}_{28}\right)^{(5)}}\left[\left(b_{i}\right)^{(5)}+\left(b_{i}^{\prime}\right)^{(5)}+\left(\hat{B}_{28}\right)^{(5)}+\left(\hat{Q}_{28}\right)^{(5)}\left(\hat{k}_{28}\right)^{(5)}\right]<1$

Where we suppose

$\left(a_{i}\right)^{(6)},\left(a_{i}^{\prime}\right)^{(6)},\left(a_{i}^{\prime \prime}\right)^{(6)},\left(b_{i}\right)^{(6)},\left(b_{i}^{\prime}\right)^{(6)},\left(b_{i}^{\prime \prime}\right)^{(6)}>0, \quad i, j=32,33,34$

(V) The functions $\left(a_{i}^{\prime \prime}\right)^{(6)},\left(b_{i}^{\prime \prime}\right)^{(6)}$ are positive continuous increasing and bounded.

Definition of $\left(p_{i}\right)^{(6)},\left(r_{i}\right)^{(6)}$ :

$\left(a_{i}^{\prime \prime}\right)^{(6)}\left(T_{33}, t\right) \leq\left(p_{i}\right)^{(6)} \leq\left(\hat{A}_{32}\right)^{(6)}$ 


$$
\left(b_{i}^{\prime \prime}\right)^{(6)}\left(\left(G_{35}\right), t\right) \leq\left(r_{i}\right)^{(6)} \leq\left(b_{i}^{\prime}\right)^{(6)} \leq\left(\widehat{B}_{32}\right)^{(6)}
$$

$$
\begin{aligned}
\lim _{T_{2} \rightarrow \infty}\left(a_{i}^{\prime \prime}\right)^{(6)}\left(T_{33}, t\right) & =\left(p_{i}\right)^{(6)} \\
\lim _{G \rightarrow \infty}\left(b_{i}^{\prime \prime}\right)^{(6)}\left(\left(G_{35}\right), t\right) & =\left(r_{i}\right)^{(6)}
\end{aligned}
$$

Definition of $\left(\hat{A}_{32}\right)^{(6)},\left(\hat{B}_{32}\right)^{(6)}$ :

Where $\left(\hat{A}_{32}\right)^{(6)},\left(\hat{B}_{32}\right)^{(6)},\left(p_{i}\right)^{(6)},\left(r_{i}\right)^{(6)}$ are positive constants and $i=32,33,34$

They satisfy Lipschitz condition:

$\left|\left(a_{i}^{\prime \prime}\right)^{(6)}\left(T_{33}^{\prime}, t\right)-\left(a_{i}^{\prime \prime}\right)^{(6)}\left(T_{33}, t\right)\right| \leq\left(\hat{k}_{32}\right)^{(6)}\left|T_{33}-T_{33}^{\prime}\right| e^{-\left(\widehat{M}_{32}\right)^{(6)} t}$

$\left|\left(b_{i}^{\prime \prime}\right)^{(6)}\left(\left(G_{35}\right)^{\prime}, t\right)-\left(b_{i}^{\prime \prime}\right)^{(6)}\left(\left(G_{35}\right),\left(T_{35}\right)\right)\right|<\left(\hat{k}_{32}\right)^{(6)}\left\|\left(G_{35}\right)-\left(G_{35}\right)^{\prime}\right\| e^{-\left(M_{32}\right)^{(6)} t}$

With the Lipschitz condition, we place a restriction on the behavior of functions $\left(a_{i}^{\prime \prime}\right)^{(6)}\left(T_{33}^{\prime}, t\right)$ $\operatorname{and}\left(a_{i}^{\prime \prime}\right)^{(6)}\left(T_{33}, t\right) .\left(T_{33}^{\prime}, t\right)$ and $\left(T_{33}, t\right)$ are points belonging to the interval $\left[\left(\hat{k}_{32}\right)^{(6)},\left(\widehat{M}_{32}\right)^{(6)}\right]$. It is to be noted that $\left(a_{i}^{\prime \prime}\right)^{(6)}\left(T_{33}, t\right)$ is uniformly continuous. In the eventuality of the fact, that if $\left(\widehat{M}_{32}\right)^{(6)}=$ 6 then the function $\left(a_{i}^{\prime \prime}\right)^{(6)}\left(T_{33}, t\right)$, the sixth augmentation coefficient would be absolutely continuous.

Definition of $\left(\widehat{M}_{32}\right)^{(6)},\left(\widehat{k}_{32}\right)^{(6)}$ :

$\left(\widehat{M}_{32}\right)^{(6)},\left(\hat{k}_{32}\right)^{(6)}$, are positive constants

$$
\frac{\left(a_{i}\right)^{(6)}}{\left(M_{32}\right)^{(6)}}, \frac{\left(b_{i}\right)^{(6)}}{\left(M_{32}\right)^{(6)}}<1
$$

Definition of $\left(\hat{P}_{32}\right)^{(6)},\left(\hat{Q}_{32}\right)^{(6)}: 1$

There exists two constants $\left(\hat{P}_{32}\right)^{(6)}$ and $\left(\hat{Q}_{32}\right)^{(6)}$ which together with $\left(\widehat{M}_{32}\right)^{(6)},\left(\hat{k}_{32}\right)^{(6)},\left(\hat{A}_{32}\right)^{(6)}$ and $\left(\hat{B}_{32}\right)^{(6)}$ and the constants $\left(a_{i}\right)^{(6)},\left(a_{i}^{\prime}\right)^{(6)},\left(b_{i}\right)^{(6)},\left(b_{i}^{\prime}\right)^{(6)},\left(p_{i}\right)^{(6)},\left(r_{i}\right)^{(6)}, i=32,33,34$, satisfy the inequalities

$\frac{1}{\left(M_{32}\right)^{(6)}}\left[\left(a_{i}\right)^{(6)}+\left(a_{i}^{\prime}\right)^{(6)}+\left(\hat{A}_{32}\right)^{(6)}+\left(\hat{P}_{32}\right)^{(6)}\left(\hat{k}_{32}\right)^{(6)}\right]<1$

$\frac{1}{\left(M_{32}\right)^{(6)}}\left[\left(b_{i}\right)^{(6)}+\left(b_{i}^{\prime}\right)^{(6)}+\left(\hat{B}_{32}\right)^{(6)}+\left(\hat{Q}_{32}\right)^{(6)}\left(\hat{k}_{32}\right)^{(6)}\right]<1$

Theorem 1: if the conditions (A)-(E)( first five conditions related to the system Boolean satisfiability problem) above are fulfilled, there exists a solution satisfying the conditions

Definition of $G_{i}(0), T_{i}(0)$ :

$$
\begin{array}{ll}
G_{i}(t) \leq\left(\hat{P}_{13}\right)^{(1)} e^{\left(M_{13}\right)^{(1)} t}, & G_{i}(0)=G_{i}^{0}>0 \\
T_{i}(t) \leq\left(\hat{Q}_{13}\right)^{(1)} e^{\left(M_{13}\right)^{(1)} t}, & T_{i}(0)=T_{i}^{0}>0
\end{array}
$$

If the conditions of second module pertaining to Knapsack problem and Hamiltonian Path Problem above are fulfilled, there exists a solution satisfying the conditions 
Definition of $G_{i}(0), T_{i}(0)$

$$
\begin{array}{ll}
G_{i}(t) \leq\left(\hat{P}_{16}\right)^{(2)} e^{\left(M_{16}\right)^{(2)} t}, & G_{i}(0)=G_{i}^{0}>0 \\
T_{i}(t) \leq\left(\hat{Q}_{16}\right)^{(2)} e^{\left(M_{16}\right)^{(2)} t}, & T_{i}(0)=T_{i}^{0}>0
\end{array}
$$

If the conditions pertaining to the third module Sub graph Isomorphism problem and Subset sum problem above are fulfilled, there exists a solution satisfying the conditions

$$
\begin{array}{cc}
G_{i}(t) \leq\left(\hat{P}_{20}\right)^{(3)} e^{\left(\widehat{M}_{20}\right)^{(3)} t}, & G_{i}(0)=G_{i}^{0}>0 \\
T_{i}(t) \leq\left(\hat{Q}_{20}\right)^{(3)} e^{\left(\widehat{M}_{20}\right)^{(3)} t}, & T_{i}(0)=T_{i}^{0}>0
\end{array}
$$

If the conditions of the fourth module Subset Sum Problem and Clique problem above are fulfilled, there exists a solution satisfying the conditions

Definition of $G_{i}(0), T_{i}(0)$ :

$$
\begin{array}{cc}
G_{i}(t) \leq\left(\hat{P}_{24}\right)^{(4)} e^{\left(M_{24}\right)^{(4)} t}, & G_{i}(0)=G_{i}^{0}>0 \\
T_{i}(t) \leq\left(\hat{Q}_{24}\right)^{(4)} e^{\left(M_{24}\right)^{(4)} t}, & T_{i}(0)=T_{i}^{0}>0
\end{array}
$$

If the conditions pertaining to the module five namely Vertex Cover Problem and Independent Set problem are fulfilled, there exists a solution satisfying the conditions

Definition of $G_{i}(0), T_{i}(0)$ :

$$
\begin{array}{ll}
G_{i}(t) \leq\left(\hat{P}_{28}\right)^{(5)} e^{\left(M_{28}\right)^{(5)} t}, & G_{i}(0)=G_{i}^{0}>0 \\
T_{i}(t) \leq\left(\hat{Q}_{28}\right)^{(5)} e^{\left(M_{28}\right)^{(5)} t}, & T_{i}(0)=T_{i}^{0}>0
\end{array}
$$

If the conditions pertaining to Dominating set problem and Graph Coloring Problem above are fulfilled, there exists a solution satisfying the conditions

Definition of $G_{i}(0), T_{i}(0)$ :

$$
\begin{array}{ll}
G_{i}(t) \leq\left(\hat{P}_{32}\right)^{(6)} e^{\left(\widehat{M}_{32}\right)^{(6)} t}, & G_{i}(0)=G_{i}^{0}>0 \\
T_{i}(t) \leq\left(\hat{Q}_{32}\right)^{(6)} e^{\left(\widehat{M}_{32}\right)^{(6)} t}, & T_{i}(0)=T_{i}^{0}>0
\end{array}
$$

\section{Proof:}

Consider operator $\mathcal{A}^{(1)}$ defined on the space of sextuples of continuous functions $G_{i}, T_{i}: \mathbb{R}_{+} \rightarrow \mathbb{R}_{+}$ which satisfy

$$
\begin{aligned}
& G_{i}(0)=G_{i}^{0}, T_{i}(0)=T_{i}^{0}, G_{i}^{0} \leq\left(\hat{P}_{13}\right)^{(1)}, T_{i}^{0} \leq\left(\hat{Q}_{13}\right)^{(1)}, \\
& 0 \leq G_{i}(t)-G_{i}^{0} \leq\left(\widehat{P}_{13}\right)^{(1)} e^{\left(M_{13}\right)^{(1)} t} \\
& 0 \leq T_{i}(t)-T_{i}^{0} \leq\left(\hat{Q}_{13}\right)^{(1)} e^{\left(M_{13}\right)^{(1)} t}
\end{aligned}
$$

By 
$\left.\bar{G}_{13}(t)=G_{13}^{0}+\int_{0}^{t}\left[\left(a_{13}\right)^{(1)} G_{14}\left(s_{(13)}\right)-\left(\left(a_{13}^{\prime}\right)^{(1)}+a_{13}^{\prime \prime}\right)^{(1)}\left(T_{14}\left(s_{(13)}\right), s_{(13)}\right)\right) G_{13}\left(s_{(13)}\right)\right] d s_{(13)}$

$\bar{G}_{14}(t)=G_{14}^{0}+\int_{0}^{t}\left[\left(a_{14}\right)^{(1)} G_{13}\left(s_{(13)}\right)-\left(\left(a_{14}^{\prime}\right)^{(1)}+\left(a_{14}^{\prime \prime}\right)^{(1)}\left(T_{14}\left(s_{(13)}\right), s_{(13)}\right)\right) G_{14}\left(s_{(13)}\right)\right] d s_{(13)}$

$\bar{G}_{15}(t)=G_{15}^{0}+\int_{0}^{t}\left[\left(a_{15}\right)^{(1)} G_{14}\left(s_{(13)}\right)-\left(\left(a_{15}^{\prime}\right)^{(1)}+\left(a_{15}^{\prime \prime}\right)^{(1)}\left(T_{14}\left(s_{(13)}\right), s_{(13)}\right)\right) G_{15}\left(s_{(13)}\right)\right] d s_{(13)}$

$\bar{T}_{13}(t)=T_{13}^{0}+\int_{0}^{t}\left[\left(b_{13}\right)^{(1)} T_{14}\left(s_{(13)}\right)-\left(\left(b_{13}^{\prime}\right)^{(1)}-\left(b_{13}^{\prime \prime}\right)^{(1)}\left(G\left(s_{(13)}\right), s_{(13)}\right)\right) T_{13}\left(s_{(13)}\right)\right] d s_{(13)}$

$\bar{T}_{14}(t)=T_{14}^{0}+\int_{0}^{t}\left[\left(b_{14}\right)^{(1)} T_{13}\left(s_{(13)}\right)-\left(\left(b_{14}^{\prime}\right)^{(1)}-\left(b_{14}^{\prime \prime}\right)^{(1)}\left(G\left(s_{(13)}\right), s_{(13)}\right)\right) T_{14}\left(s_{(13)}\right)\right] d s_{(13)}$

$\overline{\mathrm{T}}_{15}(\mathrm{t})=\mathrm{T}_{15}^{0}+\int_{0}^{t}\left[\left(b_{15}\right)^{(1)} T_{14}\left(s_{(13)}\right)-\left(\left(b_{15}^{\prime}\right)^{(1)}-\left(b_{15}^{\prime \prime}\right)^{(1)}\left(G\left(s_{(13)}\right), s_{(13)}\right)\right) T_{15}\left(s_{(13)}\right)\right] d s_{(13)}$

Where $s_{(13)}$ is the integrand that is integrated over an interval $(0, t)$

Consider operator $\mathcal{A}^{(2)}$ defined on the space of sextuples of continuous functions $G_{i}, T_{i}: \mathbb{R}_{+} \rightarrow \mathbb{R}_{+}$ which satisfy

$G_{i}(0)=G_{i}^{0}, T_{i}(0)=T_{i}^{0}, G_{i}^{0} \leq\left(\hat{P}_{16}\right)^{(2)}, T_{i}^{0} \leq\left(\hat{Q}_{16}\right)^{(2)}$,

$0 \leq G_{i}(t)-G_{i}^{0} \leq\left(\hat{P}_{16}\right)^{(2)} e^{\left(M_{16}\right)^{(2) t}}$

$0 \leq T_{i}(t)-T_{i}^{0} \leq\left(\hat{Q}_{16}\right)^{(2)} e^{\left(M_{16}\right)^{(2)} t}$

By

$\left.\bar{G}_{16}(t)=G_{16}^{0}+\int_{0}^{t}\left[\left(a_{16}\right)^{(2)} G_{17}\left(s_{(16)}\right)-\left(\left(a_{16}^{\prime}\right)^{(2)}+a_{16}^{\prime \prime}\right)^{(2)}\left(T_{17}\left(s_{(16)}\right), s_{(16)}\right)\right) G_{16}\left(s_{(16)}\right)\right] d s_{(16)}$

$\bar{G}_{17}(t)=G_{17}^{0}+\int_{0}^{t}\left[\left(a_{17}\right)^{(2)} G_{16}\left(s_{(16)}\right)-\left(\left(a_{17}^{\prime}\right)^{(2)}+\left(a_{17}^{\prime \prime}\right)^{(2)}\left(T_{17}\left(s_{(16)}\right), s_{(17)}\right)\right) G_{17}\left(s_{(16)}\right)\right] d s_{(16)}$

$\bar{G}_{18}(t)=G_{18}^{0}+\int_{0}^{t}\left[\left(a_{18}\right)^{(2)} G_{17}\left(s_{(16)}\right)-\left(\left(a_{18}^{\prime}\right)^{(2)}+\left(a_{18}^{\prime \prime}\right)^{(2)}\left(T_{17}\left(s_{(16)}\right), s_{(16)}\right)\right) G_{18}\left(s_{(16)}\right)\right] d s_{(16)}$

$\bar{T}_{16}(t)=T_{16}^{0}+\int_{0}^{t}\left[\left(b_{16}\right)^{(2)} T_{17}\left(s_{(16)}\right)-\left(\left(b_{16}^{\prime}\right)^{(2)}-\left(b_{16}^{\prime \prime}\right)^{(2)}\left(G\left(s_{(16)}\right), s_{(16)}\right)\right) T_{16}\left(s_{(16)}\right)\right] d s_{(16)}$

$\bar{T}_{17}(t)=T_{17}^{0}+\int_{0}^{t}\left[\left(b_{17}\right)^{(2)} T_{16}\left(s_{(16)}\right)-\left(\left(b_{17}^{\prime}\right)^{(2)}-\left(b_{17}^{\prime \prime}\right)^{(2)}\left(G\left(s_{(16)}\right), s_{(16)}\right)\right) T_{17}\left(s_{(16)}\right)\right] d s_{(16)}$

$\bar{T}_{18}(t)=T_{18}^{0}+\int_{0}^{t}\left[\left(b_{18}\right)^{(2)} T_{17}\left(s_{(16)}\right)-\left(\left(b_{18}^{\prime}\right)^{(2)}-\left(b_{18}^{\prime \prime}\right)^{(2)}\left(G\left(s_{(16)}\right), s_{(16)}\right)\right) T_{18}\left(s_{(16)}\right)\right] d s_{(16)}$

Where $s_{(16)}$ is the integrand that is integrated over an interval $(0, t)$

Consider operator $\mathcal{A}^{(3)}$ defined on the space of sextuples of continuous functions $G_{i}, T_{i}: \mathbb{R}_{+} \rightarrow \mathbb{R}_{+}$ which satisfy

$G_{i}(0)=G_{i}^{0}, T_{i}(0)=T_{i}^{0}, G_{i}^{0} \leq\left(\hat{P}_{20}\right)^{(3)}, T_{i}^{0} \leq\left(\hat{Q}_{20}\right)^{(3)}$,

$0 \leq G_{i}(t)-G_{i}^{0} \leq\left(\hat{P}_{20}\right)^{(3)} e^{\left(M_{20}\right)^{(3) t}}$

$0 \leq T_{i}(t)-T_{i}^{0} \leq\left(\hat{Q}_{20}\right)^{(3)} e^{\left(M_{20}\right)^{(3)} t}$

By

$\left.\bar{G}_{20}(t)=G_{20}^{0}+\int_{0}^{t}\left[\left(a_{20}\right)^{(3)} G_{21}\left(s_{(20)}\right)-\left(\left(a_{20}^{\prime}\right)^{(3)}+a_{20}^{\prime \prime}\right)^{(3)}\left(T_{21}\left(s_{(20)}\right), s_{(20)}\right)\right) G_{20}\left(s_{(20)}\right)\right] d s_{(20)}$ 
$\bar{G}_{21}(t)=G_{21}^{0}+\int_{0}^{t}\left[\left(a_{21}\right)^{(3)} G_{20}\left(s_{(20)}\right)-\left(\left(a_{21}^{\prime}\right)^{(3)}+\left(a_{21}^{\prime \prime}\right)^{(3)}\left(T_{21}\left(s_{(20)}\right), s_{(20)}\right)\right) G_{21}\left(s_{(20)}\right)\right] d s_{(20)}$

$\bar{G}_{22}(t)=G_{22}^{0}+\int_{0}^{t}\left[\left(a_{22}\right)^{(3)} G_{21}\left(s_{(20)}\right)-\left(\left(a_{22}^{\prime}\right)^{(3)}+\left(a_{22}^{\prime \prime}\right)^{(3)}\left(T_{21}\left(s_{(20)}\right), s_{(20)}\right)\right) G_{22}\left(s_{(20)}\right)\right] d s_{(20)}$

$\bar{T}_{20}(t)=T_{20}^{0}+\int_{0}^{t}\left[\left(b_{20}\right)^{(3)} T_{21}\left(s_{(20)}\right)-\left(\left(b_{20}^{\prime}\right)^{(3)}-\left(b_{20}^{\prime \prime}\right)^{(3)}\left(G\left(s_{(20)}\right), s_{(20)}\right)\right) T_{20}\left(s_{(20)}\right)\right] d s_{(20)}$

$\bar{T}_{21}(t)=T_{21}^{0}+\int_{0}^{t}\left[\left(b_{21}\right)^{(3)} T_{20}\left(s_{(20)}\right)-\left(\left(b_{21}^{\prime}\right)^{(3)}-\left(b_{21}^{\prime \prime}\right)^{(3)}\left(G\left(s_{(20)}\right), s_{(20)}\right)\right) T_{21}\left(s_{(20)}\right)\right] d s_{(20)}$

$\overline{\mathrm{T}}_{22}(\mathrm{t})=\mathrm{T}_{22}^{0}+\int_{0}^{t}\left[\left(b_{22}\right)^{(3)} T_{21}\left(s_{(20)}\right)-\left(\left(b_{22}^{\prime}\right)^{(3)}-\left(b_{22}^{\prime \prime}\right)^{(3)}\left(G\left(s_{(20)}\right), s_{(20)}\right)\right) T_{22}\left(s_{(20)}\right)\right] d s_{(20)}$

Where $s_{(20)}$ is the integrand that is integrated over an interval $(0, t)$

Proof: Consider operator $\mathcal{A}^{(4)}$ defined on the space of sextuples of continuous functions $G_{i}, T_{i}: \mathbb{R}_{+} \rightarrow$ $\mathbb{R}_{+}$which satisfy

$G_{i}(0)=G_{i}^{0}, T_{i}(0)=T_{i}^{0}, G_{i}^{0} \leq\left(\hat{P}_{24}\right)^{(4)}, T_{i}^{0} \leq\left(\hat{Q}_{24}\right)^{(4)}$,

$0 \leq G_{i}(t)-G_{i}^{0} \leq\left(\hat{P}_{24}\right)^{(4)} e^{\left(M_{24}\right)^{(4)} t}$

$0 \leq T_{i}(t)-T_{i}^{0} \leq\left(\hat{Q}_{24}\right)^{(4)} e^{\left(M_{24}\right)^{(4) t}}$

By

$$
\begin{aligned}
& \left.\bar{G}_{24}(t)=G_{24}^{0}+\int_{0}^{t}\left[\left(a_{24}\right)^{(4)} G_{25}\left(s_{(24)}\right)-\left(\left(a_{24}^{\prime}\right)^{(4)}+a_{24}^{\prime \prime}\right)^{(4)}\left(T_{25}\left(s_{(24)}\right), s_{(24)}\right)\right) G_{24}\left(s_{(24)}\right)\right] d s_{(24)} \\
& \bar{G}_{25}(t)=G_{25}^{0}+\int_{0}^{t}\left[\left(a_{25}\right)^{(4)} G_{24}\left(s_{(24)}\right)-\left(\left(a_{25}^{\prime}\right)^{(4)}+\left(a_{25}^{\prime \prime}\right)^{(4)}\left(T_{25}\left(s_{(24)}\right), s_{(24)}\right)\right) G_{25}\left(s_{(24)}\right)\right] d s_{(24)}
\end{aligned}
$$

$\bar{G}_{26}(t)=G_{26}^{0}+\int_{0}^{t}\left[\left(a_{26}\right)^{(4)} G_{25}\left(s_{(24)}\right)-\left(\left(a_{26}^{\prime}\right)^{(4)}+\left(a_{26}^{\prime \prime}\right)^{(4)}\left(T_{25}\left(s_{(24)}\right), s_{(24)}\right)\right) G_{26}\left(s_{(24)}\right)\right] d s_{(24)}$

$\bar{T}_{24}(t)=T_{24}^{0}+\int_{0}^{t}\left[\left(b_{24}\right)^{(4)} T_{25}\left(s_{(24)}\right)-\left(\left(b_{24}^{\prime}\right)^{(4)}-\left(b_{24}^{\prime \prime}\right)^{(4)}\left(G\left(s_{(24)}\right), s_{(24)}\right)\right) T_{24}\left(s_{(24)}\right)\right] d s_{(24)}$

$\bar{T}_{25}(t)=T_{25}^{0}+\int_{0}^{t}\left[\left(b_{25}\right)^{(4)} T_{24}\left(s_{(24)}\right)-\left(\left(b_{25}^{\prime}\right)^{(4)}-\left(b_{25}^{\prime \prime}\right)^{(4)}\left(G\left(s_{(24)}\right), s_{(24)}\right)\right) T_{25}\left(s_{(24)}\right)\right] d s_{(24)}$

$\overline{\mathrm{T}}_{26}(\mathrm{t})=\mathrm{T}_{26}^{0}+\int_{0}^{t}\left[\left(b_{26}\right)^{(4)} T_{25}\left(s_{(24)}\right)-\left(\left(b_{26}^{\prime}\right)^{(4)}-\left(b_{26}^{\prime \prime}\right)^{(4)}\left(G\left(s_{(24)}\right), s_{(24)}\right)\right) T_{26}\left(s_{(24)}\right)\right] d s_{(24)}$

Where $s_{(24)}$ is the integrand that is integrated over an interval $(0, t)$

Consider operator $\mathcal{A}^{(5)}$ defined on the space of sextuples of continuous functions $G_{i}, T_{i}: \mathbb{R}_{+} \rightarrow \mathbb{R}_{+}$ which satisfy

$G_{i}(0)=G_{i}^{0}, T_{i}(0)=T_{i}^{0}, G_{i}^{0} \leq\left(\hat{P}_{28}\right)^{(5)}, T_{i}^{0} \leq\left(\hat{Q}_{28}\right)^{(5)}$,

$0 \leq G_{i}(t)-G_{i}^{0} \leq\left(\hat{P}_{28}\right)^{(5)} e^{\left(M_{28}\right)^{(5) t}}$

$0 \leq T_{i}(t)-T_{i}^{0} \leq\left(\hat{Q}_{28}\right)^{(5)} e^{\left(M_{28}\right)^{(5) t}}$ 
$\left.\bar{G}_{28}(t)=G_{28}^{0}+\int_{0}^{t}\left[\left(a_{28}\right)^{(5)} G_{29}\left(s_{(28)}\right)-\left(\left(a_{28}^{\prime}\right)^{(5)}+a_{28}^{\prime \prime}\right)^{(5)}\left(T_{29}\left(s_{(28)}\right), s_{(28)}\right)\right) G_{28}\left(s_{(28)}\right)\right] d s_{(28)}$

$\bar{G}_{29}(t)=G_{29}^{0}+\int_{0}^{t}\left[\left(a_{29}\right)^{(5)} G_{28}\left(s_{(28)}\right)-\left(\left(a_{29}^{\prime}\right)^{(5)}+\left(a_{29}^{\prime \prime}\right)^{(5)}\left(T_{29}\left(s_{(28)}\right), s_{(28)}\right)\right) G_{29}\left(s_{(28)}\right)\right] d s_{(28)}$

$\bar{G}_{30}(t)=G_{30}^{0}+\int_{0}^{t}\left[\left(a_{30}\right)^{(5)} G_{29}\left(s_{(28)}\right)-\left(\left(a_{30}^{\prime}\right)^{(5)}+\left(a_{30}^{\prime \prime}\right)^{(5)}\left(T_{29}\left(s_{(28)}\right), s_{(28)}\right)\right) G_{30}\left(s_{(28)}\right)\right] d s_{(28)}$

$\bar{T}_{28}(t)=T_{28}^{0}+\int_{0}^{t}\left[\left(b_{28}\right)^{(5)} T_{29}\left(s_{(28)}\right)-\left(\left(b_{28}^{\prime}\right)^{(5)}-\left(b_{28}^{\prime \prime}\right)^{(5)}\left(G\left(s_{(28)}\right), s_{(28)}\right)\right) T_{28}\left(s_{(28)}\right)\right] d s_{(28)}$

$\bar{T}_{29}(t)=T_{29}^{0}+\int_{0}^{t}\left[\left(b_{29}\right)^{(5)} T_{28}\left(s_{(28)}\right)-\left(\left(b_{29}^{\prime}\right)^{(5)}-\left(b_{29}^{\prime \prime}\right)^{(5)}\left(G\left(s_{(28)}\right), s_{(28)}\right)\right) T_{29}\left(s_{(28)}\right)\right] d s_{(28)}$

$\overline{\mathrm{T}}_{30}(\mathrm{t})=\mathrm{T}_{30}^{0}+\int_{0}^{t}\left[\left(b_{30}\right)^{(5)} T_{29}\left(s_{(28)}\right)-\left(\left(b_{30}^{\prime}\right)^{(5)}-\left(b_{30}^{\prime \prime}\right)^{(5)}\left(G\left(s_{(28)}\right), s_{(28)}\right)\right) T_{30}\left(s_{(28)}\right)\right] d s_{(28)}$

Where $s_{(28)}$ is the integrand that is integrated over an interval $(0, t)$

Consider operator $\mathcal{A}^{(6)}$ defined on the space of sextuples of continuous functions $G_{i}, T_{i}: \mathbb{R}_{+} \rightarrow \mathbb{R}_{+}$ which satisfy

$G_{i}(0)=G_{i}^{0}, T_{i}(0)=T_{i}^{0}, G_{i}^{0} \leq\left(\hat{P}_{32}\right)^{(6)}, T_{i}^{0} \leq\left(\hat{Q}_{32}\right)^{(6)}$,

$0 \leq G_{i}(t)-G_{i}^{0} \leq\left(\hat{P}_{32}\right)^{(6)} e^{\left(M_{32}\right)^{(6) t}}$

$0 \leq T_{i}(t)-T_{i}^{0} \leq\left(\hat{Q}_{32}\right)^{(6)} e^{\left(\widehat{M}_{32}\right)^{(6) t}}$

By

$\left.\bar{G}_{32}(t)=G_{32}^{0}+\int_{0}^{t}\left[\left(a_{32}\right)^{(6)} G_{33}\left(s_{(32)}\right)-\left(\left(a_{32}^{\prime}\right)^{(6)}+a_{32}^{\prime \prime}\right)^{(6)}\left(T_{33}\left(s_{(32)}\right), s_{(32)}\right)\right) G_{32}\left(s_{(32)}\right)\right] d s_{(32)}$

$\bar{G}_{33}(t)=G_{33}^{0}+\int_{0}^{t}\left[\left(a_{33}\right)^{(6)} G_{32}\left(s_{(32)}\right)-\left(\left(a_{33}^{\prime}\right)^{(6)}+\left(a_{33}^{\prime \prime}\right)^{(6)}\left(T_{33}\left(s_{(32)}\right), s_{(32)}\right)\right) G_{33}\left(s_{(32)}\right)\right] d s_{(32)}$

$\bar{G}_{34}(t)=G_{34}^{0}+\int_{0}^{t}\left[\left(a_{34}\right)^{(6)} G_{33}\left(s_{(32)}\right)-\left(\left(a_{34}^{\prime}\right)^{(6)}+\left(a_{34}^{\prime \prime}\right)^{(6)}\left(T_{33}\left(s_{(32)}\right), s_{(32)}\right)\right) G_{34}\left(s_{(32)}\right)\right] d s_{(32)}$

$\bar{T}_{32}(t)=T_{32}^{0}+\int_{0}^{t}\left[\left(b_{32}\right)^{(6)} T_{33}\left(s_{(32)}\right)-\left(\left(b_{32}^{\prime}\right)^{(6)}-\left(b_{32}^{\prime \prime}\right)^{(6)}\left(G\left(s_{(32)}\right), s_{(32)}\right)\right) T_{32}\left(s_{(32)}\right)\right] d s_{(32)}$

$\bar{T}_{33}(t)=T_{33}^{0}+\int_{0}^{t}\left[\left(b_{33}\right)^{(6)} T_{32}\left(s_{(32)}\right)-\left(\left(b_{33}^{\prime}\right)^{(6)}-\left(b_{33}^{\prime \prime}\right)^{(6)}\left(G\left(s_{(32)}\right), s_{(32)}\right)\right) T_{33}\left(s_{(32)}\right)\right] d s_{(32)}$

$\overline{\mathrm{T}}_{34}(\mathrm{t})=\mathrm{T}_{34}^{0}+\int_{0}^{t}\left[\left(b_{34}\right)^{(6)} T_{33}\left(s_{(32)}\right)-\left(\left(b_{34}^{\prime}\right)^{(6)}-\left(b_{34}^{\prime \prime}\right)^{(6)}\left(G\left(s_{(32)}\right), s_{(32)}\right)\right) T_{34}\left(s_{(32)}\right)\right] d s_{(32)}$

Where $s_{(32)}$ is the integrand that is integrated over an interval $(0, t)$

(a) The operator $\mathcal{A}^{(1)}$ maps the space of functions satisfying into itself .Indeed it is obvious that

$$
\begin{aligned}
G_{13}(t) \leq & G_{13}^{0}+\int_{0}^{t}\left[\left(a_{13}\right)^{(1)}\left(G_{14}^{0}+\left(\hat{P}_{13}\right)^{(1)} e^{\left.\left(M_{13}\right)^{(1)} s_{(13)}\right)}\right)\right] d s_{(13)}= \\
& \left(1+\left(a_{13}\right)^{(1)} t\right) G_{14}^{0}+\frac{\left(a_{13}\right)^{(1)}\left(\hat{P}_{13}\right)^{(1)}}{\left(M_{13}\right)^{(1)}}\left(e^{\left(M_{13}\right)^{(1)} t}-1\right)
\end{aligned}
$$

From which it follows that 
$\left(G_{13}(t)-G_{13}^{0}\right) e^{-\left(M_{13}\right)^{(1)} t} \leq \frac{\left(a_{13}\right)^{(1)}}{\left(M_{13}\right)^{(1)}}\left[\left(\left(\hat{P}_{13}\right)^{(1)}+G_{14}^{0}\right) e^{\left(-\frac{\left(\hat{P}_{13}\right)^{(1)}+G_{14}^{0}}{G_{14}^{0}}\right)}+\left(\hat{P}_{13}\right)^{(1)}\right]$

$\left(G_{i}^{0}\right)$ is as defined in the statement of theorem 1

Analogous inequalities hold also for $G_{14}, G_{15}, T_{13}, T_{14}, T_{15}$

(b) The operator $\mathcal{A}^{(2)}$ maps the space of functions satisfying into itself .Indeed it is obvious that

$$
\begin{aligned}
& G_{16}(t) \leq G_{16}^{0}+\int_{0}^{t}\left[\left(a_{16}\right)^{(2)}\left(G_{17}^{0}+\left(\hat{P}_{16}\right)^{(6)} e^{\left.\left(\widehat{M}_{16}\right)^{(2)} s_{(16)}\right)}\right)\right] d s_{(16)}=\left(1+\left(a_{16}\right)^{(2)} t\right) G_{17}^{0}+ \\
& \frac{\left(a_{16}\right)^{(2)}\left(\hat{P}_{16}\right)^{(2)}}{\left(\widehat{M}_{16}\right)^{(2)}}\left(e^{\left(\widehat{M}_{16}\right)^{(2)} t}-1\right)
\end{aligned}
$$

From which it follows that

$\left(G_{16}(t)-G_{16}^{0}\right) e^{-\left(\widehat{M}_{16}\right)^{(2)} t} \leq \frac{\left(a_{16}\right)^{(2)}}{\left(M_{16}\right)^{(2)}}\left[\left(\left(\hat{P}_{16}\right)^{(2)}+G_{17}^{0}\right) e^{\left(-\frac{\left(\hat{P}_{16}\right)^{(2)}+G_{17}^{0}}{G_{17}^{0}}\right)}+\left(\hat{P}_{16}\right)^{(2)}\right]$

Analogous inequalities hold also for $G_{17}, G_{18}, T_{16}, T_{17}, T_{18}$

(a) The operator $\mathcal{A}^{(3)}$ maps the space of functions satisfying into itself .Indeed it is obvious that

$$
\begin{aligned}
G_{20}(t) \leq & G_{20}^{0}+\int_{0}^{t}\left[\left(a_{20}\right)^{(3)}\left(G_{21}^{0}+\left(\hat{P}_{20}\right)^{(3)} e^{\left(\widehat{M}_{20}\right)^{(3)} s(20)}\right)\right] d s_{(20)}= \\
& \left(1+\left(a_{20}\right)^{(3)} t\right) G_{21}^{0}+\frac{\left(a_{20}\right)^{(3)}\left(\hat{P}_{20}\right)^{(3)}}{\left(M_{20}\right)^{(3)}}\left(e^{\left(M_{20}\right)^{(3)} t}-1\right)
\end{aligned}
$$

From which it follows that

$\left(G_{20}(t)-G_{20}^{0}\right) e^{-\left(M_{20}\right)^{(3)} t} \leq \frac{\left(a_{20}\right)^{(3)}}{\left(M_{20}\right)^{(3)}}\left[\left(\left(\hat{P}_{20}\right)^{(3)}+G_{21}^{0}\right) e^{\left(-\frac{\left(\hat{P}_{20}\right)^{(3)}+G_{21}^{0}}{G_{21}^{0}}\right)}+\left(\hat{P}_{20}\right)^{(3)}\right] 277$

Analogous inequalities hold also for $G_{21}, G_{22}, T_{20}, T_{21}, T_{22} 278$

(b) The operator $\mathcal{A}^{(4)}$ maps the space of functions satisfying into itself .Indeed it is obvious that $G_{24}(t) \leq G_{24}^{0}+\int_{0}^{t}\left[\left(a_{24}\right)^{(4)}\left(G_{25}^{0}+\left(\hat{P}_{24}\right)^{(4)} e^{\left.\left(\widehat{M}_{24}\right)^{(4)} s_{(24)}\right)}\right)\right] d s_{(24)}=$

$$
\left(1+\left(a_{24}\right)^{(4)} t\right) G_{25}^{0}+\frac{\left(a_{24}\right)^{(4)}\left(\hat{P}_{24}\right)^{(4)}}{\left(\widehat{M}_{24}\right)^{(4)}}\left(e^{\left(\widehat{M}_{24}\right)^{(4)} t}-1\right)
$$

From which it follows that

$\left(G_{24}(t)-G_{24}^{0}\right) e^{-\left(M_{24}\right)^{(4)} t} \leq \frac{\left(a_{24}\right)^{(4)}}{\left(M_{24}\right)^{(4)}}\left[\left(\left(\hat{P}_{24}\right)^{(4)}+G_{25}^{0}\right) e^{\left(-\frac{\left(\hat{P}_{24}\right)^{(4)}+G_{25}^{0}}{G_{25}^{0}}\right)}+\left(\hat{P}_{24}\right)^{(4)}\right]$

$\left(G_{i}^{0}\right)$ is as defined in the statement of theorem 4

(c) The operator $\mathcal{A}^{(5)}$ maps the space of functions satisfying 35,35,36 into itself .Indeed it is obvious that

$$
\begin{gathered}
G_{28}(t) \leq G_{28}^{0}+\int_{0}^{t}\left[\left(a_{28}\right)^{(5)}\left(G_{29}^{0}+\left(\hat{P}_{28}\right)^{(5)} e^{\left(M_{28}\right)^{(5)} s(28)}\right)\right] d s_{(28)}= \\
\left(1+\left(a_{28}\right)^{(5)} t\right) G_{29}^{0}+\frac{\left(a_{28}\right)^{(5)}\left(\hat{P}_{28}\right)^{(5)}}{\left(M_{28}\right)^{(5)}}\left(e^{\left(M_{28}\right)^{(5)} t}-1\right)
\end{gathered}
$$


From which it follows that

$\left(G_{28}(t)-G_{28}^{0}\right) e^{-\left(M_{28}\right)^{(5)} t} \leq \frac{\left(a_{28}\right)^{(5)}}{\left(M_{28}\right)^{(5)}}\left[\left(\left(\hat{P}_{28}\right)^{(5)}+G_{29}^{0}\right) e^{\left(-\frac{\left(\hat{P}_{28}\right)^{(5)}+G_{29}^{0}}{G_{29}^{0}}\right)}+\left(\hat{P}_{28}\right)^{(5)}\right]$

$\left(G_{i}^{0}\right)$ is as defined in the statement of theorem 1

(d) The operator $\mathcal{A}^{(6)}$ maps the space of functions satisfying 34,35,36 into itself .Indeed it is obvious that

$$
\begin{gathered}
G_{32}(t) \leq G_{32}^{0}+\int_{0}^{t}\left[\left(a_{32}\right)^{(6)}\left(G_{33}^{0}+\left(\hat{P}_{32}\right)^{(6)} e^{\left.\left(\widehat{M}_{32}\right)^{(6)} s_{(32)}\right)}\right)\right] d s_{(32)}= \\
\left(1+\left(a_{32}\right)^{(6)} t\right) G_{33}^{0}+\frac{\left(a_{32}\right)^{(6)}\left(\hat{P}_{32}\right)^{(6)}}{\left(\widehat{M}_{32}\right)^{(6)}}\left(e^{\left(\widehat{M}_{32}\right)^{(6)} t}-1\right)
\end{gathered}
$$

From which it follows that

$\left(G_{32}(t)-G_{32}^{0}\right) e^{-\left(\widehat{M}_{32}\right)^{(6)} t} \leq \frac{\left(a_{32}\right)^{(6)}}{\left(\widehat{M}_{32}\right)^{(6)}}\left[\left(\left(\hat{P}_{32}\right)^{(6)}+G_{33}^{0}\right) e^{\left(-\frac{\left(\hat{P}_{32}\right)^{(6)}+G_{33}^{0}}{G_{33}^{0}}\right)}+\left(\hat{P}_{32}\right)^{(6)}\right]$

$\left(G_{i}^{0}\right)$ is as defined in the statement of theorem1

Analogous inequalities hold also for $G_{25}, G_{26}, T_{24}, T_{25}, T_{26}$

It is now sufficient to take $\frac{\left(a_{i}\right)^{(1)}}{\left(\bar{M}_{13}\right)^{(1)}}, \frac{\left(b_{i}\right)^{(1)}}{\left(M_{13}\right)^{(1)}}<1$ and to choose

$\left(\widehat{\mathrm{P}}_{13}\right)^{(1)}$ and $\left(\widehat{\mathrm{Q}}_{13}\right)^{(1)}$ large to have

$\frac{\left(a_{i}\right)^{(1)}}{\left(\widehat{M}_{13}\right)^{(1)}}\left[\left(\widehat{P}_{13}\right)^{(1)}+\left(\left(\hat{P}_{13}\right)^{(1)}+G_{j}^{0}\right) e^{-\left(\frac{\left(\widehat{P}_{13}\right)^{(1)}+G_{j}^{0}}{G_{j}^{0}}\right)}\right] \leq\left(\hat{P}_{13}\right)^{(1)}$

$\frac{\left(b_{i}\right)^{(1)}}{\left(\widehat{M}_{13}\right)^{(1)}}\left[\left(\left(\hat{Q}_{13}\right)^{(1)}+T_{j}^{0}\right) e^{-\left(\frac{\left(\widehat{Q}_{13}\right)^{(1)}+T_{j}^{0}}{T_{j}^{0}}\right)}+\left(\hat{Q}_{13}\right)^{(1)}\right] \leq\left(\hat{Q}_{13}\right)^{(1)}$

In order that the operator $\mathcal{A}^{(1)}$ transforms the space of sextuples of functions $G_{i}, T_{i}$ into itself

The operator $\mathcal{A}^{(1)}$ is a contraction with respect to the metric

$d\left(\left(G^{(1)}, T^{(1)}\right),\left(G^{(2)}, T^{(2)}\right)\right)=$

$\sup _{i}\left\{\max _{t \in \mathbb{R}_{+}}\left|G_{i}^{(1)}(t)-G_{i}^{(2)}(t)\right| e^{-\left(M_{13}\right)^{(1)} t}, \max _{t \in \mathbb{R}_{+}}\left|T_{i}^{(1)}(t)-T_{i}^{(2)}(t)\right| e^{-\left(M_{13}\right)^{(1)} t}\right\}$

Indeed if we denote

Definition of $\tilde{G}, \tilde{T}: \quad(\tilde{G}, \tilde{T})=\mathcal{A}^{(1)}(G, T)$

It results 
$\left|\tilde{G}_{13}^{(1)}-\tilde{G}_{i}^{(2)}\right| \leq \int_{0}^{t}\left(a_{13}\right)^{(1)}\left|G_{14}^{(1)}-G_{14}^{(2)}\right| e^{-\left(\bar{M}_{13}\right)^{(1)} s_{(13)}} e^{\left(\prod_{13}\right)^{(1)} s_{(13)}} d s_{(13)}+$

$\int_{0}^{t}\left\{\left(a_{13}^{\prime}\right)^{(1)}\left|G_{13}^{(1)}-G_{13}^{(2)}\right| e^{-\left(\bar{M}_{13}\right)^{(1)} s_{(13)}} e^{-\left(\bar{M}_{13}\right)^{(1)} s_{(13)}}+\right.$

$\left(a_{13}^{\prime \prime}\right)^{(1)}\left(T_{14}^{(1)}, s_{(13)}\right)\left|G_{13}^{(1)}-G_{13}^{(2)}\right| e^{-\left(\widehat{M}_{13}\right)^{(1)} s_{(13)}} e^{\left(\widehat{M}_{13}\right)^{(1)} s_{(13)}}+$

$\left.G_{13}^{(2)}\left|\left(a_{13}^{\prime \prime}\right)^{(1)}\left(T_{14}^{(1)}, s_{(13)}\right)-\left(a_{13}^{\prime \prime}\right)^{(1)}\left(T_{14}^{(2)}, s_{(13)}\right)\right| e^{-\left(\widehat{M}_{13}\right)^{(1)} s_{(13)}} e^{\left(\widehat{M}_{13}\right)^{(1)} s_{(13)}}\right\} d s_{(13)}$

Where $s_{(13)}$ represents integrand that is integrated over the interval $[0, t]$

From the hypotheses it follows

$\left|G^{(1)}-G^{(2)}\right| e^{-\left(\bar{M}_{13}\right)^{(1)} t} \leq$

$\frac{1}{\left(\widehat{M}_{13}\right)^{(1)}}\left(\left(a_{13}\right)^{(1)}+\left(a_{13}^{\prime}\right)^{(1)}+\left(\widehat{A}_{13}\right)^{(1)}+\left(\widehat{P}_{13}\right)^{(1)}\left(\widehat{k}_{13}\right)^{(1)}\right) d\left(\left(G^{(1)}, T^{(1)} ; G^{(2)}, T^{(2)}\right)\right)$

And analogous inequalities for $G_{i}$ and $T_{i}$. Taking into account the result follows

Remark 1: The fact that we supposed $\left(a_{13}^{\prime \prime}\right)^{(1)}$ and $\left(b_{13}^{\prime \prime}\right)^{(1)}$ depending also on $\mathrm{t}$ can be considered as not conformal with the reality, however we have put this hypothesis, in order that we can postulate condition necessary to prove the uniqueness of the solution bounded by $\left(\widehat{P}_{13}\right)^{(1)} e^{\left(\widehat{M}_{13}\right)^{(1)} t}$ and $\left(\widehat{Q}_{13}\right)^{(1)} e^{\left(\widehat{M}_{13}\right)^{(1)} t}$ respectively of $\mathbb{R}_{+}$.

If instead of proving the existence of the solution on $\mathbb{R}_{+}$, we have to prove it only on a compact then it suffices to consider that $\left(a_{i}^{\prime \prime}\right)^{(1)}$ and $\left(b_{i}^{\prime \prime}\right)^{(1)}, i=13,14,15$ depend only on $\mathrm{T}_{14}$ and respectively on $G$ (and not on $t$ ) and hypothesis can replaced by a usual Lipschitz condition.

Remark 2: There does not exist any $t$ where $G_{i}(t)=0$ and $T_{i}(t)=0$

From the governing equations of the holistic system it results

$G_{i}(t) \geq G_{i}^{0} e^{\left[-\int_{0}^{t}\left\{\left(a_{i}^{\prime}\right)^{(1)}-\left(a_{i}^{\prime \prime}\right)^{(1)}\left(T_{14}\left(s_{(13)}\right), s(13)\right)\right\} d s_{(13)}\right]} \geq 0$

$T_{i}(t) \geq T_{i}^{0} e^{\left(-\left(b_{i}^{\prime}\right)^{(1)} t\right)}>0$ for $\mathrm{t}>0$

Definition of $\left(\left(\widehat{M}_{13}\right)^{(1)}\right)_{1},\left(\left(\widehat{M}_{13}\right)^{(1)}\right)_{2}$ and $\left(\left(\widehat{M}_{13}\right)^{(1)}\right)_{3}$ :

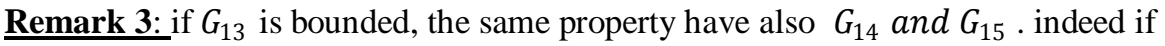

$G_{13}<\left(\widehat{M}_{13}\right)^{(1)}$ it follows $\frac{d G_{14}}{d t} \leq\left(\left(\widehat{M}_{13}\right)^{(1)}\right)_{1}-\left(a_{14}^{\prime}\right)^{(1)} G_{14}$ and by integrating

$G_{14} \leq\left(\left(\widehat{M}_{13}\right)^{(1)}\right)_{2}=G_{14}^{0}+2\left(a_{14}\right)^{(1)}\left(\left(\widehat{M}_{13}\right)^{(1)}\right)_{1} /\left(a_{14}^{\prime}\right)^{(1)}$

In the same way, one can obtain

$G_{15} \leq\left(\left(\widehat{M}_{13}\right)^{(1)}\right)_{3}=G_{15}^{0}+2\left(a_{15}\right)^{(1)}\left(\left(\widehat{M}_{13}\right)^{(1)}\right)_{2} /\left(a_{15}^{\prime}\right)^{(1)}$

If $G_{14}$ or $G_{15}$ is bounded, the same property follows for $G_{13}, G_{15}$ and $G_{13}, G_{14}$ respectively.

Remark 4: If $G_{13}$ is bounded, from below, the same property holds for $G_{14}$ and $G_{15}$. The proof is analogous with the preceding one. An analogous property is true if $G_{14}$ is bounded from below.

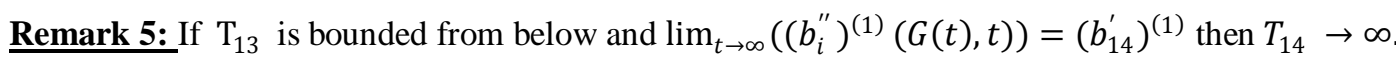


Definition of $(m)^{(1)}$ and $\varepsilon_{1}$ :

Indeed let $t_{1}$ be so that for $t>t_{1}$

$\left(b_{14}\right)^{(1)}-\left(b_{i}^{\prime \prime}\right)^{(1)}(G(t), t)<\varepsilon_{1}, T_{13}(t)>(m)^{(1)}$

Then $\frac{d T_{14}}{d t} \geq\left(a_{14}\right)^{(1)}(m)^{(1)}-\varepsilon_{1} T_{14}$ which leads to

$T_{14} \geq\left(\frac{\left(a_{14}\right)^{(1)}(m)^{(1)}}{\varepsilon_{1}}\right)\left(1-e^{-\varepsilon_{1} t}\right)+T_{14}^{0} e^{-\varepsilon_{1} t}$ If we take $t$ such that $e^{-\varepsilon_{1} t}=\frac{1}{2}$ it results

$T_{14} \geq\left(\frac{\left(a_{14}\right)^{(1)}(m)^{(1)}}{2}\right), \quad t=\log \frac{2}{\varepsilon_{1}}$ By taking now $\varepsilon_{1}$ sufficiently small one sees that $\mathrm{T}_{14}$ is unbounded.

The same property holds for $T_{15}$ if $\lim _{t \rightarrow \infty}\left(b_{15}^{\prime \prime}\right)^{(1)}(G(t), t)=\left(b_{15}^{\prime}\right)^{(1)}$

We now state a more precise theorem about the behaviors at infinity of the solutions of equations solution to the governing equations of the global system

It is now sufficient to take $\frac{\left(a_{i}\right)^{(2)}}{\left(M_{16}\right)^{(2)}}, \frac{\left(b_{i}\right)^{(2)}}{\left(M_{16}\right)^{(2)}}<1$ and to choose

$\left(\hat{P}_{16}\right)^{(2)}$ and $\left(\hat{Q}_{16}\right)^{(2)}$ large to have

$\frac{\left(a_{i}\right)^{(2)}}{\left(M_{16}\right)^{(2)}}\left[\left(\widehat{P}_{16}\right)^{(2)}+\left(\left(\hat{P}_{16}\right)^{(2)}+G_{j}^{0}\right) e^{-\left(\frac{\left(\widehat{P}_{16}\right)^{(2)}+G_{j}^{0}}{G_{j}^{0}}\right)}\right] \leq\left(\hat{P}_{16}\right)^{(2)}$

$\frac{\left(_{i}\right)^{(2)}}{\left(M_{16}\right)^{(2)}}\left[\left(\left(\hat{Q}_{16}\right)^{(2)}+T_{j}^{0}\right) e^{-\left(\frac{\left(\hat{Q}_{16}\right)^{(2)}+T_{j}^{0}}{T_{j}^{0}}\right)}+\left(\hat{Q}_{16}\right)^{(2)}\right] \leq\left(\hat{Q}_{16}\right)^{(2)}$

In order that the operator $\mathcal{A}^{(2)}$ transforms the space of sextuples of functions $G_{i}, T_{i}$ into itself

The operator $\mathcal{A}^{(2)}$ is a contraction with respect to the metric

$d\left(\left(\left(G_{19}\right)^{(1)},\left(T_{19}\right)^{(1)}\right),\left(\left(G_{19}\right)^{(2)},\left(T_{19}\right)^{(2)}\right)\right)=$

$\left.\sup _{i} \max _{t \in \mathbb{R}_{+}}\left|G_{i}^{(1)}(t)-G_{i}^{(2)}(t)\right| e^{-\left(M_{16}\right)^{(2)} t}, \max _{t \in \mathbb{R}_{+}}\left|T_{i}^{(1)}(t)-T_{i}^{(2)}(t)\right| e^{-\left(M_{16}\right)^{(2)} t}\right\}$

Indeed if we denote

Definition of $\widetilde{G_{19}}, \widetilde{T_{19}}:\left(\widetilde{G_{19}}, \widetilde{T_{19}}\right)=\mathcal{A}^{(2)}\left(G_{19}, T_{19}\right)$

It results

$\left|\tilde{G}_{16}^{(1)}-\tilde{G}_{i}^{(2)}\right| \leq \int_{0}^{t}\left(a_{16}\right)^{(2)}\left|G_{17}^{(1)}-G_{17}^{(2)}\right| e^{-\left(\widehat{M}_{16}\right)^{(2)} s_{(16)}} e^{\left(\widehat{M}_{16}\right)^{(2)} s_{(16)}} d s_{(16)}+$

$\int_{0}^{t}\left\{\left(a_{16}^{\prime}\right)^{(2)}\left|G_{16}^{(1)}-G_{16}^{(2)}\right| e^{-\left(\mathbb{M}_{16}\right)^{(2)} s_{(16)}} e^{-\left(\mathbb{M}_{16}\right)^{(2)} s_{(16)}}+\right.$

$\left(a_{16}^{\prime \prime}\right)^{(2)}\left(T_{17}^{(1)}, s_{(16)}\right)\left|G_{16}^{(1)}-G_{16}^{(2)}\right| e^{-\left(\bar{M}_{16}\right)^{(2)} s_{(16)}} e^{\left(\bar{M}_{16}\right)^{(2)} s_{(16)}}+$

$\left.G_{16}^{(2)}\left|\left(a_{16}^{\prime \prime}\right)^{(2)}\left(T_{17}^{(1)}, s_{(16)}\right)-\left(a_{16}^{\prime \prime}\right)^{(2)}\left(T_{17}^{(2)}, s_{(16)}\right)\right| e^{-\left(\prod_{16}\right)^{(2)} s_{(16)}} e^{\left(\prod_{16}\right)^{(2)} s_{(16)}}\right\} d s_{(16)}$ 
Where $s_{(16)}$ represents integrand that is integrated over the interval $[0, t]$

From the hypotheses it follows

$\left|\left(G_{19}\right)^{(1)}-\left(G_{19}\right)^{(2)}\right| \mathrm{e}^{-\left(\widehat{\mathrm{M}}_{16}\right)^{(2)} \mathrm{t}} \leq$

$\frac{1}{\left(\widehat{\mathrm{M}}_{16}\right)^{(2)}}\left(\left(a_{16}\right)^{(2)}+\left(a_{16}^{\prime}\right)^{(2)}+\left(\widehat{\mathrm{A}}_{16}\right)^{(2)}+\left(\widehat{\mathrm{P}}_{16}\right)^{(2)}\left(\widehat{k}_{16}\right)^{(2)}\right) \mathrm{d}\left(\left(\left(G_{19}\right)^{(1)},\left(T_{19}\right)^{(1)} ;\left(G_{19}\right)^{(2)},\left(T_{19}\right)^{(2)}\right)\right)$

And analogous inequalities for $\mathrm{G}_{i}$ and $\mathrm{T}_{i}$. Taking into account the hypothesis $(34,35,36)$ the result follows

Remark 1: The fact that we supposed $\left(a_{16}^{\prime \prime}\right)^{(2)}$ and $\left(b_{16}^{\prime \prime}\right)^{(2)}$ depending also on $t$ can be considered as not conformal with the reality, however we have put this hypothesis ,in order that we can postulate condition necessary to prove the uniqueness of the solution bounded by $\left(\widehat{P}_{16}\right)^{(2)} \mathrm{e}^{\left(\widehat{\mathrm{M}}_{16}\right)^{(2)} \mathrm{t}}$ and $\left(\widehat{\mathrm{Q}}_{16}\right)^{(2)} \mathrm{e}^{\left(\widehat{\mathrm{M}}_{16}\right)^{(2)} \mathrm{t}}$ respectively of $\mathbb{R}_{+}$.

If instead of proving the existence of the solution on $\mathbb{R}_{+}$, we have to prove it only on a compact then it suffices to consider that $\left(a_{i}^{\prime \prime}\right)^{(2)}$ and $\left(b_{i}^{\prime \prime}\right)^{(2)}, i=16,17,18$ depend only on $\mathrm{T}_{17}$ and respectively on $\left(G_{19}\right)$ (and not on $\mathrm{t}$ ) and hypothesis can replaced by a usual Lipschitz condition.

Remark 2: There does not exist any $\mathrm{t}$ where $\mathrm{G}_{i}(\mathrm{t})=0$ and $\mathrm{T}_{i}(\mathrm{t})=0$

From 19 to 24 it results

$\mathrm{G}_{i}(\mathrm{t}) \geq \mathrm{G}_{i}^{0} \mathrm{e}^{\left[-\int_{0}^{\mathrm{t}}\left\{\left(a_{i}^{\prime}\right)^{(2)}-\left(a_{i}^{\prime \prime}\right)^{(2)}\left(\mathrm{T}_{17}\left(s_{(16)}\right), s_{(16)}\right)\right\} \mathrm{d} s_{(16)}\right]} \geq 0$

$\mathrm{T}_{i}(\mathrm{t}) \geq \mathrm{T}_{i}^{0} \mathrm{e}^{\left(-\left(b_{i}^{\prime}\right)^{(2)} \mathrm{t}\right)}>0$ for $\mathrm{t}>0$

Definition of $\left(\left(\widehat{\mathrm{M}}_{16}\right)^{(2)}\right)_{1},\left(\left(\widehat{\mathrm{M}}_{16}\right)^{(2)}\right)_{2}$ and $\left(\left(\widehat{\mathrm{M}}_{16}\right)^{(2)}\right)_{3}$ :

Remark 3: if $G_{16}$ is bounded, the same property have also $G_{17}$ and $G_{18}$. indeed if

$\mathrm{G}_{16}<\left(\widehat{\mathrm{M}}_{16}\right)^{(2)}$ it follows $\frac{\mathrm{dG}_{17}}{\mathrm{dt}} \leq\left(\left(\widehat{\mathrm{M}}_{16}\right)^{(2)}\right)_{1}-\left(a_{17}^{\prime}\right)^{(2)} \mathrm{G}_{17}$ and by integrating

$\mathrm{G}_{17} \leq\left(\left(\widehat{\mathrm{M}}_{16}\right)^{(2)}\right)_{2}=\mathrm{G}_{17}^{0}+2\left(a_{17}\right)^{(2)}\left(\left(\widehat{\mathrm{M}}_{16}\right)^{(2)}\right)_{1} /\left(a_{17}^{\prime}\right)^{(2)}$

In the same way, one can obtain

$\mathrm{G}_{18} \leq\left(\left(\widehat{\mathrm{M}}_{16}\right)^{(2)}\right)_{3}=\mathrm{G}_{18}^{0}+2\left(a_{18}\right)^{(2)}\left(\left(\widehat{\mathrm{M}}_{16}\right)^{(2)}\right)_{2} /\left(a_{18}^{\prime}\right)^{(2)}$

If $G_{17}$ or $G_{18}$ is bounded, the same property follows for $G_{16}, G_{18}$ and $G_{16}, G_{17}$ respectively.

Remark 4: If $G_{16}$ is bounded, from below, the same property holds for $G_{17}$ and $G_{18}$. The proof is analogous with the preceding one. An analogous property is true if $G_{17}$ is bounded from below.

Remark 5: If $\mathrm{T}_{16}$ is bounded from below and $\lim _{\mathrm{t} \rightarrow \infty}\left(\left(b_{i}^{\prime \prime}\right)^{(2)}\left(\left(G_{19}\right)(\mathrm{t}), \mathrm{t}\right)\right)=\left(b_{17}^{\prime}\right)^{(2)}$ then $\mathrm{T}_{17} \rightarrow \infty$.

$\underline{\text { Definition of }}(m)^{(2)}$ and $\varepsilon_{2}$ :

Indeed let $t_{2}$ be so that for $t>t_{2}$

$\left(b_{17}\right)^{(2)}-\left(b_{i}^{\prime \prime}\right)^{(2)}\left(\left(G_{19}\right)(\mathrm{t}), \mathrm{t}\right)<\varepsilon_{2}, \mathrm{~T}_{16}(\mathrm{t})>(m)^{(2)}$

Then $\frac{\mathrm{dT}_{17}}{\mathrm{dt}} \geq\left(a_{17}\right)^{(2)}(m)^{(2)}-\varepsilon_{2} \mathrm{~T}_{17}$ which leads to 
$\mathrm{T}_{17} \geq\left(\frac{\left(a_{17}\right)^{(2)}(m)^{(2)}}{\varepsilon_{2}}\right)\left(1-\mathrm{e}^{-\varepsilon_{2} \mathrm{t}}\right)+\mathrm{T}_{17}^{0} \mathrm{e}^{-\varepsilon_{2} \mathrm{t}}$ If we take $\mathrm{t}$ such that $\mathrm{e}^{-\varepsilon_{2} \mathrm{t}}=\frac{1}{2}$ it results

$\mathrm{T}_{17} \geq\left(\frac{\left(a_{17}\right)^{(2)}(m)^{(2)}}{2}\right), \quad t=\log \frac{2}{\varepsilon_{2}}$ By taking now $\varepsilon_{2}$ sufficiently small one sees that $\mathrm{T}_{17}$ is unbounded.

The same property holds for $\mathrm{T}_{18}$ if $\lim _{t \rightarrow \infty}\left(b_{18}^{\prime \prime}\right)^{(2)}\left(\left(G_{19}\right)(\mathrm{t}), \mathrm{t}\right)=\left(b_{18}^{\prime}\right)^{(2)}$

We now state a more precise theorem about the behaviors at infinity of the solutions of equations 37 to 42

It is now sufficient to take $\frac{\left(a_{i}\right)^{(3)}}{\left(\widetilde{M}_{20}\right)^{(3)}}, \frac{\left(b_{i}\right)^{(3)}}{\left(\widetilde{M}_{20}\right)^{(3)}}<1$ and to choose

$\left(\widehat{P}_{20}\right)^{(3)}$ and $\left(\widehat{Q}_{20}\right)^{(3)}$ large to have

$\frac{\left(a_{i}\right)^{(3)}}{\left(\widehat{M}_{20}\right)^{(3)}}\left[\left(\widehat{P}_{20}\right)^{(3)}+\left(\left(\hat{P}_{20}\right)^{(3)}+G_{j}^{0}\right) e^{-\left(\frac{\left(\widehat{P}_{20}\right)^{(3)}+G_{j}^{0}}{G_{j}^{0}}\right)}\right] \leq\left(\hat{P}_{20}\right)^{(3)}$

$\frac{\left(b_{i}\right)^{(3)}}{\left(M_{20}\right)^{(3)}}\left[\left(\left(\hat{Q}_{20}\right)^{(3)}+T_{j}^{0}\right) e^{-\left(\frac{\left(\hat{Q}_{20}\right)^{(3)}+T_{j}^{0}}{T_{j}^{0}}\right)}+\left(\hat{Q}_{20}\right)^{(3)}\right] \leq\left(\hat{Q}_{20}\right)^{(3)}$

In order that the operator $\mathcal{A}^{(3)}$ transforms the space of sextuples of functions $G_{i}, T_{i}$ into itself

The operator $\mathcal{A}^{(3)}$ is a contraction with respect to the metric

$d\left(\left(\left(G_{23}\right)^{(1)},\left(T_{23}\right)^{(1)}\right),\left(\left(G_{23}\right)^{(2)},\left(T_{23}\right)^{(2)}\right)\right)=$

$\sup _{i}\left\{\max _{t \in \mathbb{R}_{+}}\left|G_{i}^{(1)}(t)-G_{i}^{(2)}(t)\right| e^{-\left(M_{20}\right)^{(3)} t}, \max _{t \in \mathbb{R}_{+}}\left|T_{i}^{(1)}(t)-T_{i}^{(2)}(t)\right| e^{-\left(\mathbb{M}_{20}\right)^{(3)} t}\right\}$

Indeed if we denote

Definition of $\widetilde{G_{23}}, \widetilde{T_{23}}:\left(\widetilde{\left(G_{23}\right)}, \widetilde{\left(T_{23}\right)}\right)=\mathcal{A}^{(3)}\left(\left(G_{23}\right),\left(T_{23}\right)\right)$

It results

$$
\begin{aligned}
& \quad\left|\tilde{G}_{20}^{(1)}-\tilde{G}_{i}^{(2)}\right| \leq \int_{0}^{t}\left(a_{20}\right)^{(3)}\left|G_{21}^{(1)}-G_{21}^{(2)}\right| e^{-\left(\bar{M}_{20}\right)^{(3)} s_{(20)}} e^{\left(\bar{M}_{20}\right)^{(3)} s_{(20)}} d s_{(20)}+ \\
& \int_{0}^{t}\left\{\left(a_{20}^{\prime}\right)^{(3)}\left|G_{20}^{(1)}-G_{20}^{(2)}\right| e^{-\left(\bar{M}_{20}\right)^{(3)} s_{(20)}} e^{-\left(\bar{M}_{20}\right)^{(3)} s_{(20)}}+\right. \\
& \left(a_{20}^{\prime \prime}\right)^{(3)}\left(T_{21}^{(1)}, s_{(20)}\right)\left|G_{20}^{(1)}-G_{20}^{(2)}\right| e^{-\left(\bar{M}_{20}\right)^{(3)} s_{(20)}} e^{\left(\bar{M}_{20}\right)^{(3)} s_{(20)}}+ \\
& \left.G_{20}^{(2)}\left|\left(a_{20}^{\prime \prime}\right)^{(3)}\left(T_{21}^{(1)}, s_{(20)}\right)-\left(a_{20}^{\prime \prime}\right)^{(3)}\left(T_{21}^{(2)}, s_{(20)}\right)\right| e^{-\left(\bar{M}_{20}\right)^{(3)} s_{(20)}} e^{\left(\bar{M}_{20}\right)^{(3)} s_{(20)}}\right\} d s_{(20)}
\end{aligned}
$$

Where $s_{(20)}$ represents integrand that is integrated over the interval $[0, \mathrm{t}]$

From the hypotheses it follows

$\left|G^{(1)}-G^{(2)}\right| e^{-\left(\bar{M}_{20}\right)^{(3)} t} \leq$

$\frac{1}{\left(\widehat{M}_{20}\right)^{(3)}}\left(\left(a_{20}\right)^{(3)}+\left(a_{20}^{\prime}\right)^{(3)}+\left(\widehat{A}_{20}\right)^{(3)}+\left(\widehat{P}_{20}\right)^{(3)}\left(\widehat{k}_{20}\right)^{(3)}\right) d\left(\left(\left(G_{23}\right)^{(1)},\left(T_{23}\right)^{(1)} ;\left(G_{23}\right)^{(2)},\left(T_{23}\right)^{(2)}\right)\right)$

And analogous inequalities for $G_{i}$ and $T_{i}$. Taking into account the hypothesis the result follows 
Remark 1: The fact that we supposed $\left(a_{20}^{\prime \prime}\right)^{(3)}$ and $\left(b_{20}^{\prime \prime}\right)^{(3)}$ depending also on $t$ can be considered as not 284 conformal with the reality, however we have put this hypothesis ,in order that we can postulate condition necessary to prove the uniqueness of the solution bounded by $\left(\widehat{P}_{20}\right)^{(3)} e^{\left(\bar{M}_{20}\right)^{(3)} t}$ and $\left(\widehat{Q}_{20}\right)^{(3)} e^{\left(\bar{M}_{20}\right)^{(3)} t}$ respectively of $\mathbb{R}_{+}$.

If instead of proving the existence of the solution on $\mathbb{R}_{+}$, we have to prove it only on a compact then it suffices to consider that $\left(a_{i}^{\prime \prime}\right)^{(3)}$ and $\left(b_{i}^{\prime \prime}\right)^{(3)}, i=20,21,22$ depend only on $\mathrm{T}_{21}$ and respectively on $\left(G_{23}\right)$ (and not on $t$ ) and hypothesis can replaced by a usual Lipschitz condition.

$\underline{\text { Remark 2: }}$ There does not exist any $t$ where $G_{i}(t)=0$ and $T_{i}(t)=0$

From 19 to 24 it results

$G_{i}(t) \geq G_{i}^{0} e^{\left[-\int_{0}^{t}\left\{\left(a_{i}^{\prime}\right)^{(3)}-\left(a_{i}^{\prime \prime}\right)^{(3)}\left(T_{21}\left(s_{(20)}\right), s_{(20)}\right)\right\} d s_{(20)}\right]} \geq 0$

$T_{i}(t) \geq T_{i}^{0} e^{\left(-\left(b_{i}^{\prime}\right)^{(3)} t\right)}>0$ for $\mathrm{t}>0$

Definition of $\left(\left(\widehat{M}_{20}\right)^{(3)}\right)_{1},\left(\left(\widehat{M}_{20}\right)^{(3)}\right)_{2}$ and $\left(\left(\widehat{M}_{20}\right)^{(3)}\right)_{3}$ :

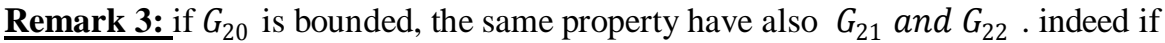

$G_{20}<\left(\widehat{M}_{20}\right)^{(3)}$ it follows $\frac{d G_{21}}{d t} \leq\left(\left(\widehat{M}_{20}\right)^{(3)}\right)_{1}-\left(a_{21}^{\prime}\right)^{(3)} G_{21}$ and by integrating

$G_{21} \leq\left(\left(\widehat{M}_{20}\right)^{(3)}\right)_{2}=G_{21}^{0}+2\left(a_{21}\right)^{(3)}\left(\left(\widehat{M}_{20}\right)^{(3)}\right)_{1} /\left(a_{21}^{\prime}\right)^{(3)}$

In the same way, one can obtain

$G_{22} \leq\left(\left(\widehat{M}_{20}\right)^{(3)}\right)_{3}=G_{22}^{0}+2\left(a_{22}\right)^{(3)}\left(\left(\widehat{M}_{20}\right)^{(3)}\right)_{2} /\left(a_{22}^{\prime}\right)^{(3)}$

If $G_{21}$ or $G_{22}$ is bounded, the same property follows for $G_{20}, G_{22}$ and $G_{20}, G_{21}$ respectively.

Remark 4: If $G_{20}$ is bounded, from below, the same property holds for $G_{21}$ and $G_{22}$. The proof is analogous with the preceding one. An analogous property is true if $G_{21}$ is bounded from below.

Remark 5: If $\mathrm{T}_{20}$ is bounded from below and $\lim _{t \rightarrow \infty}\left(\left(b_{i}^{\prime \prime}\right)^{(3)}\left(\left(G_{23}\right)(t), t\right)\right)=\left(b_{21}^{\prime}\right)^{(3)}$ then $T_{21} \rightarrow \infty$.

$\underline{\text { Definition of }}(\mathrm{m})^{(3)}$ and $\varepsilon_{3}$ :

Indeed let $t_{3}$ be so that for $t>t_{3}$

$\left(b_{21}\right)^{(3)}-\left(b_{i}^{\prime \prime}\right)^{(3)}\left(\left(G_{23}\right)(t), t\right)<\varepsilon_{3}, T_{20}(t)>(m)^{(3)}$

Then $\frac{d T_{21}}{d t} \geq\left(a_{21}\right)^{(3)}(m)^{(3)}-\varepsilon_{3} T_{21}$ which leads to

$T_{21} \geq\left(\frac{\left(a_{21}\right)^{(3)}(m)^{(3)}}{\varepsilon_{3}}\right)\left(1-e^{-\varepsilon_{3} t}\right)+T_{21}^{0} e^{-\varepsilon_{3} t}$ If we take $t$ such that $e^{-\varepsilon_{3} t}=\frac{1}{2}$ it results

$T_{21} \geq\left(\frac{\left(a_{21}\right)^{(3)}(m)^{(3)}}{2}\right), \quad t=\log \frac{2}{\varepsilon_{3}}$ By taking now $\varepsilon_{3}$ sufficiently small one sees that $\mathrm{T}_{21}$ is unbounded. The same property holds for $T_{22}$ if $\lim _{t \rightarrow \infty}\left(b_{22}^{\prime \prime}\right)^{(3)}\left(\left(G_{23}\right)(t), t\right)=\left(b_{22}^{\prime}\right)^{(3)}$

We now state a more precise theorem about the behaviors at infinity of the solutions:

It is now sufficient to take $\frac{\left(a_{i}\right)^{(4)}}{\left(M_{24}\right)^{(4)}}, \frac{\left(b_{i}\right)^{(4)}}{\left(M_{24}\right)^{(4)}}<1$ and to choose 
$\left(\widehat{\mathrm{P}}_{24}\right)^{(4)}$ and $\left(\widehat{\mathrm{Q}}_{24}\right)^{(4)}$ large to have

$\frac{\left(a_{i}\right)^{(4)}}{\left(M_{24}\right)^{(4)}}\left[\left(\widehat{P}_{24}\right)^{(4)}+\left(\left(\hat{P}_{24}\right)^{(4)}+G_{j}^{0}\right) e^{-\left(\frac{\left(\widehat{P}_{24}\right)^{(4)}+G_{j}^{0}}{G_{j}^{0}}\right)}\right] \leq\left(\hat{P}_{24}\right)^{(4)}$

$\frac{\left(b_{i}\right)^{(4)}}{\left(\widehat{M}_{24}\right)^{(4)}}\left[\left(\left(\hat{Q}_{24}\right)^{(4)}+T_{j}^{0}\right) e^{-\left(\frac{\left(\hat{Q}_{24}\right)^{(4)}+T_{j}^{0}}{T_{j}^{0}}\right)}+\left(\hat{Q}_{24}\right)^{(4)}\right] \leq\left(\hat{Q}_{24}\right)^{(4)}$

In order that the operator $\mathcal{A}^{(4)}$ transforms the space of sextuples of functions $G_{i}, T_{i}$ into itself

The operator $\mathcal{A}^{(4)}$ is a contraction with respect to the metric

$d\left(\left(\left(G_{27}\right)^{(1)},\left(T_{27}\right)^{(1)}\right),\left(\left(G_{27}\right)^{(2)},\left(T_{27}\right)^{(2)}\right)\right)=$

$\sup _{i}\left\{\max _{t \in \mathbb{R}_{+}}\left|G_{i}^{(1)}(t)-G_{i}^{(2)}(t)\right| e^{-\left(M_{24}\right)^{(4)} t}, \max _{t \in \mathbb{R}_{+}}\left|T_{i}^{(1)}(t)-T_{i}^{(2)}(t)\right| e^{-\left(\mathbb{M}_{24}\right)^{(4)} t}\right\}$

Indeed if we denote

Definition of $\overline{\left(G_{27}\right)}, \overline{\left(T_{27}\right)}: \quad\left(\widetilde{\left(G_{27}\right)}, \overline{\left(T_{27}\right)}\right)=\mathcal{A}^{(4)}\left(\left(G_{27}\right),\left(T_{27}\right)\right)$

It results

$$
\begin{aligned}
& \left|\tilde{G}_{24}^{(1)}-\tilde{G}_{i}^{(2)}\right| \leq \int_{0}^{t}\left(a_{24}\right)^{(4)}\left|G_{25}^{(1)}-G_{25}^{(2)}\right| e^{-\left(\bar{M}_{24}\right)^{(4)} s(24)} e^{\left(\bar{M}_{24}\right)^{(4)} s_{(24)}} d s_{(24)}+ \\
& \int_{0}^{t}\left\{\left(a_{24}^{\prime}\right)^{(4)}\left|G_{24}^{(1)}-G_{24}^{(2)}\right| e^{-\left(\bar{M}_{24}\right)^{(4)} s(24)} e^{-\left(\bar{M}_{24}\right)^{(4)} s(24)}+\right. \\
& \left(a_{24}^{\prime \prime}\right)^{(4)}\left(T_{25}^{(1)}, s_{(24)}\right)\left|G_{24}^{(1)}-G_{24}^{(2)}\right| e^{-\left(\bar{M}_{24}\right)^{(4)} s_{(24)}} e^{\left(\bar{M}_{24}\right)^{(4)} s(24)}+ \\
& \left.\quad G_{24}^{(2)}\left|\left(a_{24}^{\prime \prime}\right)^{(4)}\left(T_{25}^{(1)}, s_{(24)}\right)-\left(a_{24}^{\prime \prime}\right)^{(4)}\left(T_{25}^{(2)}, s_{(24)}\right)\right| e^{-\left(\bar{M}_{24}\right)^{(4)} s(24)} e^{\left(\bar{M}_{24}\right)^{(4)} s_{(24)}}\right\} d s_{(24)}
\end{aligned}
$$

Where $s_{(24)}$ represents integrand that is integrated over the interval $[0, t]$

From the hypotheses it follows

$\left|\left(G_{27}\right)^{(1)}-\left(G_{27}\right)^{(2)}\right| e^{-\left(\widehat{M}_{24}\right)^{(4)} t} \leq$

$\frac{1}{\left(\widehat{M}_{24}\right)^{(4)}}\left(\left(a_{24}\right)^{(4)}+\left(a_{24}^{\prime}\right)^{(4)}+\left(\widehat{A}_{24}\right)^{(4)}+\left(\widehat{P}_{24}\right)^{(4)}\left(\widehat{k}_{24}\right)^{(4)}\right) d\left(\left(\left(G_{27}\right)^{(1)},\left(T_{27}\right)^{(1)} ;\left(G_{27}\right)^{(2)},\left(T_{27}\right)^{(2)}\right)\right)$

And analogous inequalities for $G_{i}$ and $T_{i}$. Taking into account the hypothesis the result follows

Remark 1: The fact that we supposed $\left(a_{24}^{\prime \prime}\right)^{(4)}$ and $\left(b_{24}^{\prime \prime}\right)^{(4)}$ depending also on $t$ can be considered as not 298 conformal with the reality, however we have put this hypothesis ,in order that we can postulate condition necessary to prove the uniqueness of the solution bounded by $\left(\widehat{P}_{24}\right)^{(4)} e^{\left(\bar{M}_{24}\right)^{(4)} t}$ and $\left(\widehat{Q}_{24}\right)^{(4)} e^{\left(\widehat{M}_{24}\right)^{(4)} t}$ respectively of $\mathbb{R}_{+}$.

If instead of proving the existence of the solution on $\mathbb{R}_{+}$, we have to prove it only on a compact then it suffices to consider that $\left(a_{i}^{\prime \prime}\right)^{(4)}$ and $\left(b_{i}^{\prime \prime}\right)^{(4)}, i=24,25,26$ depend only on $\mathrm{T}_{25}$ and respectively on 
$\left(G_{27}\right)($ and not on $t)$ and hypothesis can replaced by a usual Lipschitz condition.

Remark 2: There does not exist any $t$ where $G_{i}(t)=0$ and $T_{i}(t)=0$

From 19 to 24 it results

$G_{i}(t) \geq G_{i}^{0} e^{\left[-\int_{0}^{t}\left\{\left(a_{i}^{\prime}\right)^{(4)}-\left(a_{i}^{\prime \prime}\right)^{(4)}\left(T_{25}\left(s_{(24)}\right), s_{(24)}\right)\right\} d s_{(24)}\right]} \geq 0$

$T_{i}(t) \geq T_{i}^{0} e^{\left(-\left(b_{i}^{\prime}\right)^{(4)} t\right)}>0$ for $\mathrm{t}>0$

Definition of $\left(\left(\widehat{M}_{24}\right)^{(4)}\right)_{1^{\prime}}\left(\left(\widehat{M}_{24}\right)^{(4)}\right)_{2}$ and $\left(\left(\widehat{M}_{24}\right)^{(4)}\right)_{3}$ :

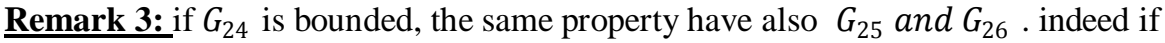

$G_{24}<\left(\widehat{M}_{24}\right)^{(4)}$ it follows $\frac{d G_{25}}{d t} \leq\left(\left(\widehat{M}_{24}\right)^{(4)}\right)_{1}-\left(a_{25}^{\prime}\right)^{(4)} G_{25}$ and by integrating

$G_{25} \leq\left(\left(\widehat{M}_{24}\right)^{(4)}\right)_{2}=G_{25}^{0}+2\left(a_{25}\right)^{(4)}\left(\left(\widehat{M}_{24}\right)^{(4)}\right)_{1} /\left(a_{25}^{\prime}\right)^{(4)}$

In the same way, one can obtain

$G_{26} \leq\left(\left(\widehat{M}_{24}\right)^{(4)}\right)_{3}=G_{26}^{0}+2\left(a_{26}\right)^{(4)}\left(\left(\widehat{M}_{24}\right)^{(4)}\right)_{2} /\left(a_{26}^{\prime}\right)^{(4)}$

If $G_{25}$ or $G_{26}$ is bounded, the same property follows for $G_{24}, G_{26}$ and $G_{24}, G_{25}$ respectively.

Remark 4: If $G_{24}$ is bounded, from below, the same property holds for $G_{25}$ and $G_{26}$. The proof is analogous with the preceding one. An analogous property is true if $G_{25}$ is bounded from below.

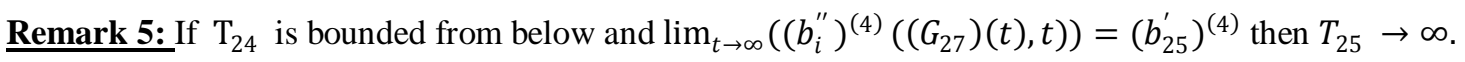

Definition of $(m)^{(4)}$ and $\varepsilon_{4}$ :

Indeed let $t_{4}$ be so that for $t>t_{4}$

$\left(b_{25}\right)^{(4)}-\left(b_{i}^{\prime \prime}\right)^{(4)}\left(\left(G_{27}\right)(t), t\right)<\varepsilon_{4}, T_{24}(t)>(m)^{(4)}$

Then $\frac{d T_{25}}{d t} \geq\left(a_{25}\right)^{(4)}(m)^{(4)}-\varepsilon_{4} T_{25}$ which leads to

$T_{25} \geq\left(\frac{\left(a_{25}\right)^{(4)}(m)^{(4)}}{\varepsilon_{4}}\right)\left(1-e^{-\varepsilon_{4} t}\right)+T_{25}^{0} e^{-\varepsilon_{4} t}$ If we take $t$ such that $e^{-\varepsilon_{4} t}=\frac{1}{2}$ it results

$T_{25} \geq\left(\frac{\left(a_{25}\right)^{(4)}(m)^{(4)}}{2}\right), \quad t=\log \frac{2}{\varepsilon_{4}}$ By taking now $\varepsilon_{4}$ sufficiently small one sees that $\mathrm{T}_{25}$ is unbounded.

The same property holds for $T_{26}$ if $\lim _{t \rightarrow \infty}\left(b_{26}^{\prime \prime}\right)^{(4)}\left(\left(G_{27}\right)(t), t\right)=\left(b_{26}^{\prime}\right)^{(4)}$

We now state a more precise theorem about the behaviors at infinity of the solutions ;

Analogous inequalities hold also for $G_{29}, G_{30}, T_{28}, T_{29}, T_{30}$

It is now sufficient to take $\frac{\left(a_{i}\right)^{(5)}}{\left(M_{28}\right)^{(5)}}, \frac{\left(b_{i}\right)^{(5)}}{\left(M_{28}\right)^{(5)}}<1$ and to choose

$\left(\widehat{\mathrm{P}}_{28}\right)^{(5)}$ and $\left(\widehat{\mathrm{Q}}_{28}\right)^{(5)}$ large to have 
$\frac{\left(a_{i}\right)^{(5)}}{\left(\widehat{M}_{28}\right)^{(5)}}\left[\left(\widehat{P}_{28}\right)^{(5)}+\left(\left(\hat{P}_{28}\right)^{(5)}+G_{j}^{0}\right) e^{-\left(\frac{\left(\widehat{P}_{28}\right)^{(5)}+G_{j}^{0}}{G_{j}^{0}}\right)}\right] \leq\left(\hat{P}_{28}\right)^{(5)}$

$\frac{\left(b_{i}\right)^{(5)}}{\left(M_{28}\right)^{(5)}}\left[\left(\left(\hat{Q}_{28}\right)^{(5)}+T_{j}^{0}\right) e^{-\left(\frac{\left(\hat{Q}_{28}\right)^{(5)}+T_{j}^{0}}{T_{j}^{0}}\right)}+\left(\hat{Q}_{28}\right)^{(5)}\right] \leq\left(\hat{Q}_{28}\right)^{(5)}$

In order that the operator $\mathcal{A}^{(5)}$ transforms the space of sextuples of functions $G_{i}, T_{i}$ into itself

The operator $\mathcal{A}^{(5)}$ is a contraction with respect to the metric

$d\left(\left(\left(G_{31}\right)^{(1)},\left(T_{31}\right)^{(1)}\right),\left(\left(G_{31}\right)^{(2)},\left(T_{31}\right)^{(2)}\right)\right)=$

$\left.\sup _{i} \max _{t \in \mathbb{R}_{+}}\left|G_{i}^{(1)}(t)-G_{i}^{(2)}(t)\right| e^{-\left(M_{28}\right)^{(5)} t}, \max _{t \in \mathbb{R}_{+}}\left|T_{i}^{(1)}(t)-T_{i}^{(2)}(t)\right| e^{-\left(M_{28}\right)^{(5)} t}\right\}$

Indeed if we denote

Definition of $\widetilde{\left(G_{31}\right)}, \widetilde{\left(T_{31}\right)}: \quad\left(\widetilde{\left(G_{31}\right)}, \widetilde{\left(T_{31}\right)}\right)=\mathcal{A}^{(5)}\left(\left(G_{31}\right),\left(T_{31}\right)\right)$

It results

$$
\begin{aligned}
& \left|\tilde{G}_{28}^{(1)}-\tilde{G}_{i}^{(2)}\right| \leq \int_{0}^{t}\left(a_{28}\right)^{(5)}\left|G_{29}^{(1)}-G_{29}^{(2)}\right| e^{-\left(\bar{M}_{28}\right)^{(5)} s_{(28)}} e^{\left(\bar{M}_{28}\right)^{(5)} s_{(28)}} d s_{(28)}+ \\
& \int_{0}^{t}\left\{\left(a_{28}^{\prime}\right)^{(5)}\left|G_{28}^{(1)}-G_{28}^{(2)}\right| e^{-\left(\bar{M}_{28}\right)^{(5)} s_{(28)}} e^{-\left(\bar{M}_{28}\right)^{(5)} s_{(28)}}+\right. \\
& \left(a_{28}^{\prime \prime}\right)^{(5)}\left(T_{29}^{(1)}, s_{(28)}\right)\left|G_{28}^{(1)}-G_{28}^{(2)}\right| e^{-\left(\bar{M}_{28}\right)^{(5)} s_{(28)}} e^{\left(\bar{M}_{28}\right)^{(5)} s_{(28)}}+ \\
& \left.G_{28}^{(2)}\left|\left(a_{28}^{\prime \prime}\right)^{(5)}\left(T_{29}^{(1)}, s_{(28)}\right)-\left(a_{28}^{\prime \prime}\right)^{(5)}\left(T_{29}^{(2)}, s_{(28)}\right)\right| e^{-\left(\widehat{M}_{28}\right)^{(5)} s_{(28)}} e^{\left(\widehat{M}_{28}\right)^{(5)} s_{(28)}}\right\} d s_{(28)}
\end{aligned}
$$

Where $s_{(28)}$ represents integrand that is integrated over the interval $[0, t]$

From the hypotheses it follows

$\left|\left(G_{31}\right)^{(1)}-\left(G_{31}\right)^{(2)}\right| e^{-\left(\bar{M}_{28}\right)^{(5)} t} \leq$

$\frac{1}{\left(\widehat{M}_{28}\right)^{(5)}}\left(\left(a_{28}\right)^{(5)}+\left(a_{28}^{\prime}\right)^{(5)}+\left(\widehat{A}_{28}\right)^{(5)}+\left(\widehat{P}_{28}\right)^{(5)}\left(\widehat{k}_{28}\right)^{(5)}\right) d\left(\left(\left(G_{31}\right)^{(1)},\left(T_{31}\right)^{(1)} ;\left(G_{31}\right)^{(2)},\left(T_{31}\right)^{(2)}\right)\right)$

And analogous inequalities for $G_{i}$ and $T_{i}$. Taking into account the hypothesis $(35,35,36)$ the result follows

Remark 1: The fact that we supposed $\left(a_{28}^{\prime \prime}\right)^{(5)}$ and $\left(b_{28}^{\prime \prime}\right)^{(5)}$ depending also on $\mathrm{t}$ can be considered as not conformal with the reality, however we have put this hypothesis ,in order that we can postulate condition necessary to prove the uniqueness of the solution bounded by $\left(\widehat{P}_{28}\right)^{(5)} e^{\left(\widehat{M}_{28}\right)^{(5)} t}$ and $\left(\widehat{Q}_{28}\right)^{(5)} e^{\left(\widehat{M}_{28}\right)^{(5)} t}$ respectively of $\mathbb{R}_{+}$.

If instead of proving the existence of the solution on $\mathbb{R}_{+}$, we have to prove it only on a compact then it suffices to consider that $\left(a_{i}^{\prime \prime}\right)^{(5)}$ and $\left(b_{i}^{\prime \prime}\right)^{(5)}, i=28,29,30$ depend only on $\mathrm{T}_{29}$ and respectively on $\left(G_{31}\right)$ (and not on $t$ ) and hypothesis can replaced by a usual Lipschitz condition. 
$\underline{\text { Remark 2: }}$ There does not exist any $t$ where $G_{i}(t)=0$ and $T_{i}(t)=0$

$G_{i}(t) \geq G_{i}^{0} e^{\left[-\int_{0}^{t}\left\{\left(a_{i}^{\prime}\right)^{(5)}-\left(a_{i}^{\prime \prime}\right)^{(5)}\left(T_{29}\left(s_{(28)}\right), s_{(28)}\right)\right\} d s_{(28)}\right]} \geq 0$

$T_{i}(t) \geq T_{i}^{0} e^{\left(-\left(b_{i}^{\prime}\right)^{(5)} t\right)}>0$ for $\mathrm{t}>0$

Definition of $\left(\left(\widehat{M}_{28}\right)^{(5)}\right)_{1},\left(\left(\widehat{M}_{28}\right)^{(5)}\right)_{2}$ and $\left(\left(\widehat{M}_{28}\right)^{(5)}\right)_{3}$ :

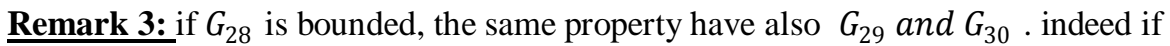

$G_{28}<\left(\widehat{M}_{28}\right)^{(5)}$ it follows $\frac{d G_{29}}{d t} \leq\left(\left(\widehat{M}_{28}\right)^{(5)}\right)_{1}-\left(a_{29}^{\prime}\right)^{(5)} G_{29}$ and by integrating

$G_{29} \leq\left(\left(\widehat{M}_{28}\right)^{(5)}\right)_{2}=G_{29}^{0}+2\left(a_{29}\right)^{(5)}\left(\left(\widehat{M}_{28}\right)^{(5)}\right)_{1} /\left(a_{29}^{\prime}\right)^{(5)}$

In the same way, one can obtain

$G_{30} \leq\left(\left(\widehat{M}_{28}\right)^{(5)}\right)_{3}=G_{30}^{0}+2\left(a_{30}\right)^{(5)}\left(\left(\widehat{M}_{28}\right)^{(5)}\right)_{2} /\left(a_{30}^{\prime}\right)^{(5)}$

If $G_{29}$ or $G_{30}$ is bounded, the same property follows for $G_{28}, G_{30}$ and $G_{28}, G_{29}$ respectively.

Remark 4: If $G_{28}$ is bounded, from below, the same property holds for $G_{29}$ and $G_{30}$. The proof is analogous with the preceding one. An analogous property is true if $G_{29}$ is bounded from below.

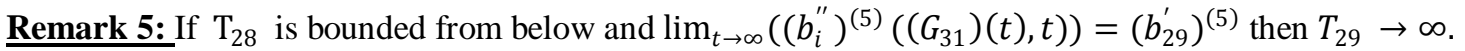

Definition of $(m)^{(5)}$ and $\varepsilon_{5}$ :

Indeed let $t_{5}$ be so that for $t>t_{5}$

$$
\left(b_{29}\right)^{(5)}-\left(b_{i}^{\prime \prime}\right)^{(5)}\left(\left(G_{31}\right)(t), t\right)<\varepsilon_{5}, T_{28}(t)>(m)^{(5)}
$$

Then $\frac{d T_{29}}{d t} \geq\left(a_{29}\right)^{(5)}(m)^{(5)}-\varepsilon_{5} T_{29}$ which leads to

$T_{29} \geq\left(\frac{\left(a_{29}\right)^{(5)}(m)^{(5)}}{\varepsilon_{5}}\right)\left(1-e^{-\varepsilon_{5} t}\right)+T_{29}^{0} e^{-\varepsilon_{5} t}$ If we take $t$ such that $e^{-\varepsilon_{5} t}=\frac{1}{2}$ it results

$T_{29} \geq\left(\frac{\left(a_{29}\right)^{(5)}(m)^{(5)}}{2}\right), \quad t=\log \frac{2}{\varepsilon_{5}}$ By taking now $\varepsilon_{5}$ sufficiently small one sees that $\mathrm{T}_{29}$ is unbounded.

The same property holds for $T_{30}$ if $\lim _{t \rightarrow \infty}\left(b_{30}^{\prime \prime}\right)^{(5)}\left(\left(G_{31}\right)(t), t\right)=\left(b_{30}^{\prime}\right)^{(5)}$

We now state a more precise theorem about the behaviors at infinity of the solutions;

Analogous inequalities hold also for $G_{33}, G_{34}, T_{32}, T_{33}, T_{34}$

It is now sufficient to take $\frac{\left(a_{i}\right)^{(6)}}{\left(M_{32}\right)^{(6)}}, \frac{\left(^{(}\right)^{(6)}}{\left(M_{32}\right)^{(6)}}<1$ and to choose

$\left(\widehat{\mathrm{P}}_{32}\right)^{(6)}$ and $\left(\widehat{\mathrm{Q}}_{32}\right)^{(6)}$ large to have

$\frac{\left(a_{i}\right)^{(6)}}{\left(M_{32}\right)^{(6)}}\left[\left(\widehat{P}_{32}\right)^{(6)}+\left(\left(\hat{P}_{32}\right)^{(6)}+G_{j}^{0}\right) e^{-\left(\frac{\left(\widehat{P}_{32}\right)^{(6)}+G_{j}^{0}}{G_{j}^{0}}\right)}\right] \leq\left(\hat{P}_{32}\right)^{(6)}$ 
$\frac{\left(b_{i}\right)^{(6)}}{\left(\hat{M}_{32}\right)^{(6)}}\left[\left(\left(\hat{Q}_{32}\right)^{(6)}+T_{j}^{0}\right) e^{-\left(\frac{\left(\hat{Q}_{32}\right)^{(6)}+T_{j}^{0}}{T_{j}^{0}}\right)}+\left(\hat{Q}_{32}\right)^{(6)}\right] \leq\left(\hat{Q}_{32}\right)^{(6)}$

In order that the operator $\mathcal{A}^{(6)}$ transforms the space of sextuples of functions $G_{i}, T_{i}$ into itself

The operator $\mathcal{A}^{(6)}$ is a contraction with respect to the metric

$d\left(\left(\left(G_{35}\right)^{(1)},\left(T_{35}\right)^{(1)}\right),\left(\left(G_{35}\right)^{(2)},\left(T_{35}\right)^{(2)}\right)\right)=$

$\sup _{i}\left\{\max _{t \in \mathbb{R}_{+}}\left|G_{i}^{(1)}(t)-G_{i}^{(2)}(t)\right| e^{-\left(M_{32}\right)^{(6)} t}, \max _{t \in \mathbb{R}_{+}}\left|T_{i}^{(1)}(t)-T_{i}^{(2)}(t)\right| e^{-\left(M_{32}\right)^{(6)} t}\right\}$

Indeed if we denote

Definition of $\widetilde{\left(G_{35}\right)}, \widetilde{\left(T_{35}\right)}: \quad\left(\widetilde{\left(G_{35}\right)}, \widetilde{\left(T_{35}\right)}\right)=\mathcal{A}^{(6)}\left(\left(G_{35}\right),\left(T_{35}\right)\right)$

It results

$\left|\tilde{G}_{32}^{(1)}-\tilde{G}_{i}^{(2)}\right| \leq \int_{0}^{t}\left(a_{32}\right)^{(6)}\left|G_{33}^{(1)}-G_{33}^{(2)}\right| e^{-\left(\widehat{M}_{32}\right)^{(6)} s_{(32)}} e^{\left(\widehat{M}_{32}\right)^{(6)} s_{(32)}} d s_{(32)}+$

$\int_{0}^{t}\left\{\left(a_{32}^{\prime}\right)^{(6)}\left|G_{32}^{(1)}-G_{32}^{(2)}\right| e^{-\left(\widehat{M}_{32}\right)^{(6)} s_{(32)}} e^{-\left(\widehat{M}_{32}\right)^{(6)} s_{(32)}}+\right.$

$\left(a_{32}^{\prime \prime}\right)^{(6)}\left(T_{33}^{(1)}, s_{(32)}\right)\left|G_{32}^{(1)}-G_{32}^{(2)}\right| e^{-\left(\bar{M}_{32}\right)^{(6)} s_{(32)}} e^{\left(\bar{M}_{32}\right)^{(6)} s_{(32)}}+$

$\left.G_{32}^{(2)}\left|\left(a_{32}^{\prime \prime}\right)^{(6)}\left(T_{33}^{(1)}, s_{(32)}\right)-\left(a_{32}^{\prime \prime}\right)^{(6)}\left(T_{33}^{(2)}, s_{(32)}\right)\right| e^{-\left(\widehat{M}_{32}\right)^{(6)} s_{(32)}} e^{\left(\widehat{M}_{32}\right)^{(6)} s_{(32)}}\right\} d s_{(32)}$

Where $s_{(32)}$ represents integrand that is integrated over the interval $[0, \mathrm{t}]$

From the hypotheses it follows

$\left|\left(G_{35}\right)^{(1)}-\left(G_{35}\right)^{(2)}\right| e^{-\left(M_{32}\right)^{(6)} t} \leq$

$\frac{1}{\left(\widehat{M}_{32}\right)^{(6)}}\left(\left(a_{32}\right)^{(6)}+\left(a_{32}^{\prime}\right)^{(6)}+\left(\widehat{A}_{32}\right)^{(6)}+\left(\widehat{P}_{32}\right)^{(6)}\left(\widehat{k}_{32}\right)^{(6)}\right) d\left(\left(\left(G_{35}\right)^{(1)},\left(T_{35}\right)^{(1)} ;\left(G_{35}\right)^{(2)},\left(T_{35}\right)^{(2)}\right)\right)$

And analogous inequalities for $G_{i}$ and $T_{i}$. Taking into account the hypothesis the result follows

Remark 1: The fact that we supposed $\left(a_{32}^{\prime \prime}\right)^{(6)}$ and $\left(b_{32}^{\prime \prime}\right)^{(6)}$ depending also on $\mathrm{t}$ can be considered as not conformal with the reality, however we have put this hypothesis ,in order that we can postulate condition necessary to prove the uniqueness of the solution bounded by $\left(\widehat{P}_{32}\right)^{(6)} e^{\left(\widehat{M}_{32}\right)^{(6)} t}$ and $\left(\widehat{Q}_{32}\right)^{(6)} e^{\left(\widehat{M}_{32}\right)^{(6)} t}$ respectively of $\mathbb{R}_{+}$.

If instead of proving the existence of the solution on $\mathbb{R}_{+}$, we have to prove it only on a compact then it suffices to consider that $\left(a_{i}^{\prime \prime}\right)^{(6)}$ and $\left(b_{i}^{\prime \prime}\right)^{(6)}, i=32,33,34$ depend only on $\mathrm{T}_{33}$ and respectively on $\left(G_{35}\right)$ (and not on $t$ ) and hypothesis can replaced by a usual Lipschitz condition.

Remark 2: There does not exist any $t$ where $G_{i}(t)=0$ and $T_{i}(t)=0$

From governing equations it results 
$G_{i}(t) \geq G_{i}^{0} e^{\left[-\int_{0}^{t}\left\{\left(a_{i}^{\prime}\right)^{(6)}-\left(a_{i}^{\prime \prime}\right)^{(6)}\left(T_{33}\left(s_{(32)}\right), s_{(32)}\right)\right\} d s_{(32)}\right]} \geq 0$

$T_{i}(t) \geq T_{i}^{0} e^{\left(-\left(b_{i}^{\prime}\right)^{(6)} t\right)}>0$ for $\mathrm{t}>0$

Definition of $\left(\left(\widehat{M}_{32}\right)^{(6)}\right)_{1},\left(\left(\widehat{M}_{32}\right)^{(6)}\right)_{2}$ and $\left(\left(\widehat{M}_{32}\right)^{(6)}\right)_{3}$ :

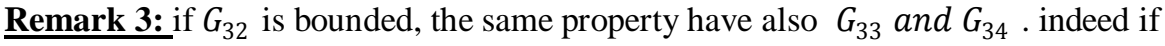

$G_{32}<\left(\widehat{M}_{32}\right)^{(6)}$ it follows $\frac{d G_{33}}{d t} \leq\left(\left(\widehat{M}_{32}\right)^{(6)}\right)_{1}-\left(a_{33}^{\prime}\right)^{(6)} G_{33}$ and by integrating

$G_{33} \leq\left(\left(\widehat{M}_{32}\right)^{(6)}\right)_{2}=G_{33}^{0}+2\left(a_{33}\right)^{(6)}\left(\left(\widehat{M}_{32}\right)^{(6)}\right)_{1} /\left(a_{33}^{\prime}\right)^{(6)}$

In the same way, one can obtain

$G_{34} \leq\left(\left(\widehat{M}_{32}\right)^{(6)}\right)_{3}=G_{34}^{0}+2\left(a_{34}\right)^{(6)}\left(\left(\widehat{M}_{32}\right)^{(6)}\right)_{2} /\left(a_{34}^{\prime}\right)^{(6)}$

If $G_{33}$ or $G_{34}$ is bounded, the same property follows for $G_{32}, G_{34}$ and $G_{32}, G_{33}$ respectively.

Remark 4: If $G_{32}$ is bounded, from below, the same property holds for $G_{33}$ and $G_{34}$. The proof is 325 analogous with the preceding one. An analogous property is true if $G_{33}$ is bounded from below.

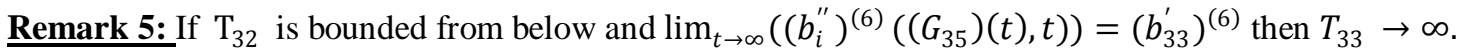

Definition of $(m)^{(6)}$ and $\varepsilon_{6}$ :

Indeed let $t_{6}$ be so that for $t>t_{6}$

$$
\left(b_{33}\right)^{(6)}-\left(b_{i}^{\prime \prime}\right)^{(6)}\left(\left(G_{35}\right)(t), t\right)<\varepsilon_{6}, T_{32}(t)>(m)^{(6)}
$$

Then $\frac{d T_{33}}{d t} \geq\left(a_{33}\right)^{(6)}(m)^{(6)}-\varepsilon_{6} T_{33}$ which leads to

$T_{33} \geq\left(\frac{\left(a_{33}\right)^{(6)}(m)^{(6)}}{\varepsilon_{6}}\right)\left(1-e^{-\varepsilon_{6} t}\right)+T_{33}^{0} e^{-\varepsilon_{6} t}$ If we take $t$ such that $e^{-\varepsilon_{6} t}=\frac{1}{2}$ it results

$T_{33} \geq\left(\frac{\left(a_{33}\right)^{(6)}(m)^{(6)}}{2}\right), \quad t=\log \frac{2}{\varepsilon_{6}}$ By taking now $\varepsilon_{6}$ sufficiently small one sees that $\mathrm{T}_{33}$ is unbounded.

The same property holds for $T_{34}$ if $\lim _{t \rightarrow \infty}\left(b_{34}^{\prime \prime}\right)^{(6)}\left(\left(G_{35}\right)(t), t(t), t\right)=\left(b_{34}^{\prime}\right)^{(6)}$

We now state a more precise theorem about the behaviors at infinity of the solutions

\section{Behavior of the solutions}

Theorem 2: If we denote and define

Definition of $\left(\sigma_{1}\right)^{(1)},\left(\sigma_{2}\right)^{(1)},\left(\tau_{1}\right)^{(1)},\left(\tau_{2}\right)^{(1)}$ :

(a) $\left.\sigma_{1}\right)^{(1)},\left(\sigma_{2}\right)^{(1)},\left(\tau_{1}\right)^{(1)},\left(\tau_{2}\right)^{(1)}$ four constants satisfying

$$
\begin{aligned}
& -\left(\sigma_{2}\right)^{(1)} \leq-\left(a_{13}^{\prime}\right)^{(1)}+\left(a_{14}^{\prime}\right)^{(1)}-\left(a_{13}^{\prime \prime}\right)^{(1)}\left(T_{14}, t\right)+\left(a_{14}^{\prime \prime}\right)^{(1)}\left(T_{14}, t\right) \leq-\left(\sigma_{1}\right)^{(1)} \\
& -\left(\tau_{2}\right)^{(1)} \leq-\left(b_{13}^{\prime}\right)^{(1)}+\left(b_{14}^{\prime}\right)^{(1)}-\left(b_{13}^{\prime \prime}\right)^{(1)}(G, t)-\left(b_{14}^{\prime \prime}\right)^{(1)}(G, t) \leq-\left(\tau_{1}\right)^{(1)}
\end{aligned}
$$

Definition of $\left(v_{1}\right)^{(1)},\left(v_{2}\right)^{(1)},\left(u_{1}\right)^{(1)},\left(u_{2}\right)^{(1)}, v^{(1)}, u^{(1)}$ : 
(b) By $\left(v_{1}\right)^{(1)}>0,\left(v_{2}\right)^{(1)}<0$ and respectively $\left(u_{1}\right)^{(1)}>0,\left(u_{2}\right)^{(1)}<0$ the roots of the equations $\left(a_{14}\right)^{(1)}\left(v^{(1)}\right)^{2}+\left(\sigma_{1}\right)^{(1)} v^{(1)}-\left(a_{13}\right)^{(1)}=0$ and $\left(b_{14}\right)^{(1)}\left(u^{(1)}\right)^{2}+\left(\tau_{1}\right)^{(1)} u^{(1)}-\left(b_{13}\right)^{(1)}=0$

Definition of $\left(\bar{v}_{1}\right)^{(1)},,\left(\bar{v}_{2}\right)^{(1)},\left(\bar{u}_{1}\right)^{(1)},\left(\bar{u}_{2}\right)^{(1)}$ :

By $\left(\bar{v}_{1}\right)^{(1)}>0,\left(\bar{v}_{2}\right)^{(1)}<0$ and respectively $\left(\bar{u}_{1}\right)^{(1)}>0,\left(\bar{u}_{2}\right)^{(1)}<0$ the roots of the equations $\left(a_{14}\right)^{(1)}\left(v^{(1)}\right)^{2}+\left(\sigma_{2}\right)^{(1)} v^{(1)}-\left(a_{13}\right)^{(1)}=0$ and $\left(b_{14}\right)^{(1)}\left(u^{(1)}\right)^{2}+\left(\tau_{2}\right)^{(1)} u^{(1)}-\left(b_{13}\right)^{(1)}=0$

Definition of $\left(m_{1}\right)^{(1)},\left(m_{2}\right)^{(1)},\left(\mu_{1}\right)^{(1)},\left(\mu_{2}\right)^{(1)},\left(v_{0}\right)^{(1)}$ :-

(c) If we define $\left(m_{1}\right)^{(1)},\left(m_{2}\right)^{(1)},\left(\mu_{1}\right)^{(1)},\left(\mu_{2}\right)^{(1)}$ by

$$
\begin{aligned}
& \left(m_{2}\right)^{(1)}=\left(v_{0}\right)^{(1)},\left(m_{1}\right)^{(1)}=\left(v_{1}\right)^{(1)}, \text { if }\left(v_{0}\right)^{(1)}<\left(v_{1}\right)^{(1)} \\
& \quad\left(m_{2}\right)^{(1)}=\left(v_{1}\right)^{(1)},\left(m_{1}\right)^{(1)}=\left(\bar{v}_{1}\right)^{(1)}, \text { if }\left(v_{1}\right)^{(1)}<\left(v_{0}\right)^{(1)}<\left(\bar{v}_{1}\right)^{(1)}, \\
& \text { and }\left(v_{0}\right)^{(1)}=\frac{G_{13}^{0}}{G_{14}^{0}} \\
& \left(m_{2}\right)^{(1)}=\left(v_{1}\right)^{(1)},\left(m_{1}\right)^{(1)}=\left(v_{0}\right)^{(1)}, \text { if }\left(\bar{v}_{1}\right)^{(1)}<\left(v_{0}\right)^{(1)}
\end{aligned}
$$

and analogously

$$
\begin{aligned}
& \left(\mu_{2}\right)^{(1)}=\left(u_{0}\right)^{(1)},\left(\mu_{1}\right)^{(1)}=\left(u_{1}\right)^{(1)}, \text { if }\left(u_{0}\right)^{(1)}<\left(u_{1}\right)^{(1)} \\
& \left(\mu_{2}\right)^{(1)}=\left(u_{1}\right)^{(1)},\left(\mu_{1}\right)^{(1)}=\left(\bar{u}_{1}\right)^{(1)}, \text { if }\left(u_{1}\right)^{(1)}<\left(u_{0}\right)^{(1)}<\left(\bar{u}_{1}\right)^{(1)}, \\
& \text { and }\left(u_{0}\right)^{(1)}=\frac{T_{13}^{0}}{T_{14}^{0}}
\end{aligned}
$$$$
\left(\mu_{2}\right)^{(1)}=\left(u_{1}\right)^{(1)},\left(\mu_{1}\right)^{(1)}=\left(u_{0}\right)^{(1)}, \text { if }\left(\bar{u}_{1}\right)^{(1)}<\left(u_{0}\right)^{(1)} \text { where }\left(u_{1}\right)^{(1)},\left(\bar{u}_{1}\right)^{(1)}
$$

are defined above

Then the solution satisfies the inequalities

$$
G_{13}^{0} e^{\left(\left(S_{1}\right)^{(1)}-\left(p_{13}\right)^{(1)}\right) t} \leq G_{13}(t) \leq G_{13}^{0} e^{\left(S_{1}\right)^{(1)} t}
$$

where $\left(p_{i}\right)^{(1)}$ is defined above

$$
\begin{aligned}
& \frac{1}{\left(m_{1}\right)^{(1)}} G_{13}^{0} e^{\left(\left(S_{1}\right)^{(1)}-\left(p_{13}\right)^{(1)}\right) t} \leq G_{14}(t) \leq \frac{1}{\left(m_{2}\right)^{(1)}} G_{13}^{0} e^{\left(S_{1}\right)^{(1)} t} \\
& \frac{\left(a_{15}\right)^{(1)} G_{13}^{0}}{\left(m_{1}\right)^{(1)}\left(\left(_{1}\right)^{(1)}-\left(p_{13}\right)^{(1)}-\left(S_{2}\right)^{(1)}\right)}\left[e^{\left(\left(S_{1}\right)^{(1)}-\left(p_{13}\right)^{(1)}\right) t}-e^{-\left(S_{2}\right)^{(1)} t}\right]+G_{15}^{0} e^{-\left(S_{2}\right)^{(1)} t} \leq G_{15}(t) \leq \\
& \left.\frac{\left(a_{15}\right)^{(1)} G_{13}^{0}}{\left(m_{2}\right)^{(1)}\left(\left(S_{1}\right)^{(1)}-\left(a_{15}^{\prime}\right)^{(1)}\right)}\left[e^{\left(S_{1}\right)^{(1)} t}-e^{-\left(a_{15}^{\prime}\right)^{(1)} t}\right]+G_{15}^{0} e^{-\left(a_{15}^{\prime}\right)^{(1)} t}\right) \\
& T_{13}^{0} e^{\left(R_{1}\right)^{(1)} t} \leq T_{13}(t) \leq T_{13}^{0} e^{\left(\left(R_{1}\right)^{(1)}+\left(r_{13}\right)^{(1)}\right) t} \\
& \frac{1}{\left(\mu_{1}\right)^{(1)}} T_{13}^{0} e^{\left(R_{1}\right)^{(1)} t} \leq T_{13}(t) \leq \frac{1}{\left(\mu_{2}\right)^{(1)}} T_{13}^{0} e^{\left(\left(R_{1}\right)^{(1)}+\left(r_{13}\right)^{(1)}\right) t} \\
& \frac{\left(b_{15}\right)^{(1)} T_{13}^{0}}{\left(\mu_{1}\right)^{(1)}\left(\left(R_{1}\right)^{(1)}-\left(b_{15}^{\prime}\right)^{(1)}\right)}\left[e^{\left(R_{1}\right)^{(1)} t}-e^{-\left(b_{15}^{\prime}\right)^{(1)} t}\right]+T_{15}^{0} e^{-\left(b_{15}^{\prime}\right)^{(1)} t} \leq T_{15}(t) \leq
\end{aligned}
$$


$\frac{\left(a_{15}\right)^{(1)} T_{13}^{0}}{\left(\mu_{2}\right)^{(1)}\left(\left(R_{1}\right)^{(1)}+\left(r_{13}\right)^{(1)}+\left(R_{2}\right)^{(1)}\right)}\left[e^{\left(\left(R_{1}\right)^{(1)}+\left(r_{13}\right)^{(1)}\right) t}-e^{-\left(R_{2}\right)^{(1)} t}\right]+T_{15}^{0} e^{-\left(R_{2}\right)^{(1)} t}$

Definition of $\left(S_{1}\right)^{(1)},\left(S_{2}\right)^{(1)},\left(R_{1}\right)^{(1)},\left(R_{2}\right)^{(1)}$ :-

Where $\left(S_{1}\right)^{(1)}=\left(a_{13}\right)^{(1)}\left(m_{2}\right)^{(1)}-\left(a_{13}^{\prime}\right)^{(1)}$

$$
\begin{aligned}
& \left(S_{2}\right)^{(1)}=\left(a_{15}\right)^{(1)}-\left(p_{15}\right)^{(1)} \\
& \left(R_{1}\right)^{(1)}=\left(b_{13}\right)^{(1)}\left(\mu_{2}\right)^{(1)}-\left(b_{13}^{\prime}\right)^{(1)} \\
& \left(R_{2}\right)^{(1)}=\left(b_{15}^{\prime}\right)^{(1)}-\left(r_{15}\right)^{(1)}
\end{aligned}
$$

\section{Behavior of the solutions}

Theorem 2: If we denote and define

Definition of $\left(\sigma_{1}\right)^{(2)},\left(\sigma_{2}\right)^{(2)},\left(\tau_{1}\right)^{(2)},\left(\tau_{2}\right)^{(2)}$ :

(d) $\left.\sigma_{1}\right)^{(2)},\left(\sigma_{2}\right)^{(2)},\left(\tau_{1}\right)^{(2)},\left(\tau_{2}\right)^{(2)}$ four constants satisfying

$-\left(\sigma_{2}\right)^{(2)} \leq-\left(a_{16}^{\prime}\right)^{(2)}+\left(a_{17}^{\prime}\right)^{(2)}-\left(a_{16}^{\prime \prime}\right)^{(2)}\left(\mathrm{T}_{17}, t\right)+\left(a_{17}^{\prime \prime}\right)^{(2)}\left(\mathrm{T}_{17}, t\right) \leq-\left(\sigma_{1}\right)^{(2)}$

$-\left(\tau_{2}\right)^{(2)} \leq-\left(b_{16}^{\prime}\right)^{(2)}+\left(b_{17}^{\prime}\right)^{(2)}-\left(b_{16}^{\prime \prime}\right)^{(2)}\left(\left(G_{19}\right), t\right)-\left(b_{17}^{\prime \prime}\right)^{(2)}\left(\left(G_{19}\right), t\right) \leq-\left(\tau_{1}\right)^{(2)}$

Definition of $\left(v_{1}\right)^{(2)},\left(v_{2}\right)^{(2)},\left(u_{1}\right)^{(2)},\left(u_{2}\right)^{(2)}$ :

By $\left(v_{1}\right)^{(2)}>0,\left(v_{2}\right)^{(2)}<0$ and respectively $\left(u_{1}\right)^{(2)}>0,\left(u_{2}\right)^{(2)}<0$ the roots

(e) of the equations $\left(a_{17}\right)^{(2)}\left(v^{(2)}\right)^{2}+\left(\sigma_{1}\right)^{(2)} v^{(2)}-\left(a_{16}\right)^{(2)}=0$

and $\left(b_{14}\right)^{(2)}\left(u^{(2)}\right)^{2}+\left(\tau_{1}\right)^{(2)} u^{(2)}-\left(b_{16}\right)^{(2)}=0$ and

Definition of $\left(\bar{v}_{1}\right)^{(2)},\left(\bar{v}_{2}\right)^{(2)},\left(\bar{u}_{1}\right)^{(2)},\left(\bar{u}_{2}\right)^{(2)}$ :

By $\left(\bar{v}_{1}\right)^{(2)}>0,\left(\bar{v}_{2}\right)^{(2)}<0$ and respectively $\left(\bar{u}_{1}\right)^{(2)}>0,\left(\bar{u}_{2}\right)^{(2)}<0$ the

roots of the equations $\left(a_{17}\right)^{(2)}\left(v^{(2)}\right)^{2}+\left(\sigma_{2}\right)^{(2)} v^{(2)}-\left(a_{16}\right)^{(2)}=0$

and $\left(b_{17}\right)^{(2)}\left(u^{(2)}\right)^{2}+\left(\tau_{2}\right)^{(2)} u^{(2)}-\left(b_{16}\right)^{(2)}=0$

Definition of $\left(m_{1}\right)^{(2)},\left(m_{2}\right)^{(2)},\left(\mu_{1}\right)^{(2)},\left(\mu_{2}\right)^{(2)}:-$

(f) If we define $\left(m_{1}\right)^{(2)},\left(m_{2}\right)^{(2)},\left(\mu_{1}\right)^{(2)},\left(\mu_{2}\right)^{(2)}$ by

$\left(m_{2}\right)^{(2)}=\left(v_{0}\right)^{(2)},\left(m_{1}\right)^{(2)}=\left(v_{1}\right)^{(2)}$, if $\left(v_{0}\right)^{(2)}<\left(v_{1}\right)^{(2)}$

$\left(m_{2}\right)^{(2)}=\left(v_{1}\right)^{(2)},\left(m_{1}\right)^{(2)}=\left(\bar{v}_{1}\right)^{(2)}$, if $\left(v_{1}\right)^{(2)}<\left(v_{0}\right)^{(2)}<\left(\bar{v}_{1}\right)^{(2)}$,

and

$\left(v_{0}\right)^{(2)}=\frac{\mathrm{G}_{16}^{0}}{\mathrm{G}_{17}^{0}}$

$\left(m_{2}\right)^{(2)}=\left(v_{1}\right)^{(2)},\left(m_{1}\right)^{(2)}=\left(v_{0}\right)^{(2)}$, if $\left(\bar{v}_{1}\right)^{(2)}<\left(v_{0}\right)^{(2)}$ 


$$
\begin{aligned}
& \left(\mu_{2}\right)^{(2)}=\left(u_{0}\right)^{(2)},\left(\mu_{1}\right)^{(2)}=\left(u_{1}\right)^{(2)}, \text { if }\left(u_{0}\right)^{(2)}<\left(u_{1}\right)^{(2)} \\
& \left(\mu_{2}\right)^{(2)}=\left(u_{1}\right)^{(2)},\left(\mu_{1}\right)^{(2)}=\left(\bar{u}_{1}\right)^{(2)}, \text { if }\left(u_{1}\right)^{(2)}<\left(u_{0}\right)^{(2)}<\left(\bar{u}_{1}\right)^{(2)}, \\
& \text { and }\left(u_{0}\right)^{(2)}=\frac{\mathrm{T}_{16}^{0}}{\mathrm{~T}_{17}^{0}}
\end{aligned}
$$$$
\left(\mu_{2}\right)^{(2)}=\left(u_{1}\right)^{(2)},\left(\mu_{1}\right)^{(2)}=\left(u_{0}\right)^{(2)}, \text { if }\left(\bar{u}_{1}\right)^{(2)}<\left(u_{0}\right)^{(2)}
$$

Then the solution satisfies the inequalities

$$
\mathrm{G}_{16}^{0} \mathrm{e}^{\left(\left(\mathrm{S}_{1}\right)^{(2)}-\left(p_{16}\right)^{(2)}\right) \mathrm{t}} \leq G_{16}(t) \leq \mathrm{G}_{16}^{0} \mathrm{e}^{\left(\mathrm{S}_{1}\right)^{(2)} \mathrm{t}}
$$

$\left(p_{i}\right)^{(2)}$ is defined by equation above

$$
\begin{aligned}
& \frac{1}{\left(m_{1}\right)^{(2)}} \mathrm{G}_{16}^{0} \mathrm{e}^{\left(\left(\mathrm{S}_{1}\right)^{(2)}-\left(p_{16}\right)^{(2)}\right) \mathrm{t}} \leq G_{17}(t) \leq \frac{1}{\left(m_{2}\right)^{(2)}} \mathrm{G}_{16}^{0} \mathrm{e}^{\left(\mathrm{S}_{1}\right)^{(2)} \mathrm{t}} \\
& \left(\frac{\left(a_{18}\right)^{(2)} \mathrm{G}_{16}^{0}}{\left.\left(m_{1}\right)^{(2)}\left(\mathrm{S}_{1}\right)^{(2)}-\left(p_{16}\right)^{(2)}-\left(\mathrm{S}_{2}\right)^{(2)}\right)}\left[\mathrm{e}^{\left(\left(\mathrm{S}_{1}\right)^{(2)}-\left(p_{16}\right)^{(2)}\right) \mathrm{t}}-\mathrm{e}^{-\left(\mathrm{S}_{2}\right)^{(2)} \mathrm{t}}\right]+\mathrm{G}_{18}^{0} \mathrm{e}^{-\left(\mathrm{S}_{2}\right)^{(2)} \mathrm{t}} \leq \mathrm{G}_{18}(t) \leq\right. \\
& \left.\frac{\left(a_{18}\right)^{(2)} \mathrm{G}_{16}^{0}}{\left(m_{2}\right)^{(2)}\left(\left(\mathrm{S}_{1}\right)^{(2)}-\left(a_{18}^{\prime}\right)^{(2)}\right)}\left[\mathrm{e}^{\left(\mathrm{S}_{1}\right)^{(2)} \mathrm{t}}-\mathrm{e}^{-\left(a_{18}^{\prime}\right)^{(2)} \mathrm{t}}\right]+\mathrm{G}_{18}^{0} \mathrm{e}^{-\left(a_{18}^{\prime}\right)^{(2)} \mathrm{t}}\right)
\end{aligned}
$$$$
\mathrm{T}_{16}^{0} \mathrm{e}^{\left(\mathrm{R}_{1}\right)^{(2)} t} \leq T_{16}(t) \leq \mathrm{T}_{16}^{0} \mathrm{e}^{\left(\left(\mathrm{R}_{1}\right)^{(2)}+\left(r_{16}\right)^{(2)}\right) t}
$$

$\frac{1}{\left(\mu_{1}\right)^{(2)}} \mathrm{T}_{16}^{0} \mathrm{e}^{\left(\mathrm{R}_{1}\right)^{(2)} t} \leq T_{16}(t) \leq \frac{1}{\left(\mu_{2}\right)^{(2)}} \mathrm{T}_{16}^{0} \mathrm{e}^{\left(\left(\mathrm{R}_{1}\right)^{(2)}+\left(r_{16}\right)^{(2)}\right) t}$

$\frac{\left(b_{18}\right)^{(2)} \mathrm{T}_{16}^{0}}{\left(\mu_{1}\right)^{(2)}\left(\left(\mathrm{R}_{1}\right)^{(2)}-\left(b_{18}^{\prime}\right)^{(2)}\right)}\left[\mathrm{e}^{\left(\mathrm{R}_{1}\right)^{(2)} t}-\mathrm{e}^{-\left(b_{18}^{\prime}\right)^{(2)} t}\right]+\mathrm{T}_{18}^{0} \mathrm{e}^{-\left(b_{18}^{\prime}\right)^{(2)} t} \leq T_{18}(t) \leq$

$\frac{\left(a_{18}\right)^{(2)} \mathrm{T}_{16}^{0}}{\left(\mu_{2}\right)^{(2)}\left(\left(\mathrm{R}_{1}\right)^{(2)}+\left(r_{16}\right)^{(2)}+\left(\mathrm{R}_{2}\right)^{(2)}\right)}\left[\mathrm{e}^{\left(\left(\mathrm{R}_{1}\right)^{(2)}+\left(r_{16}\right)^{(2)}\right) t}-\mathrm{e}^{-\left(\mathrm{R}_{2}\right)^{(2)} t}\right]+\mathrm{T}_{18}^{0} \mathrm{e}^{-\left(\mathrm{R}_{2}\right)^{(2)} t}$

Definition of $\left(\mathrm{S}_{1}\right)^{(2)},\left(\mathrm{S}_{2}\right)^{(2)},\left(\mathrm{R}_{1}\right)^{(2)},\left(\mathrm{R}_{2}\right)^{(2)}:-$

Where $\left(\mathrm{S}_{1}\right)^{(2)}=\left(a_{16}\right)^{(2)}\left(m_{2}\right)^{(2)}-\left(a_{16}^{\prime}\right)^{(2)}$

$$
\begin{aligned}
& \left(\mathrm{S}_{2}\right)^{(2)}=\left(a_{18}\right)^{(2)}-\left(p_{18}\right)^{(2)} \\
& \left(R_{1}\right)^{(2)}=\left(b_{16}\right)^{(2)}\left(\mu_{2}\right)^{(1)}-\left(b_{16}^{\prime}\right)^{(2)} \\
& \left(\mathrm{R}_{2}\right)^{(2)}=\left(b_{18}^{\prime}\right)^{(2)}-\left(r_{18}\right)^{(2)}
\end{aligned}
$$

Behavior of the solutions

Theorem 2: If we denote and define

Definition of $\left(\sigma_{1}\right)^{(3)},\left(\sigma_{2}\right)^{(3)},\left(\tau_{1}\right)^{(3)},\left(\tau_{2}\right)^{(3)}$ :

(a) $\left.\sigma_{1}\right)^{(3)},\left(\sigma_{2}\right)^{(3)},\left(\tau_{1}\right)^{(3)},\left(\tau_{2}\right)^{(3)}$ four constants satisfying

$$
\begin{aligned}
& -\left(\sigma_{2}\right)^{(3)} \leq-\left(a_{20}^{\prime}\right)^{(3)}+\left(a_{21}^{\prime}\right)^{(3)}-\left(a_{20}^{\prime \prime}\right)^{(3)}\left(T_{21}, t\right)+\left(a_{21}^{\prime \prime}\right)^{(3)}\left(T_{21}, t\right) \leq-\left(\sigma_{1}\right)^{(3)} \\
& -\left(\tau_{2}\right)^{(3)} \leq-\left(b_{20}^{\prime}\right)^{(3)}+\left(b_{21}^{\prime}\right)^{(3)}-\left(b_{20}^{\prime \prime}\right)^{(3)}(G, t)-\left(b_{21}^{\prime \prime}\right)^{(3)}\left(\left(G_{23}\right), t\right) \leq-\left(\tau_{1}\right)^{(3)}
\end{aligned}
$$

Definition of $\left(v_{1}\right)^{(3)},\left(v_{2}\right)^{(3)},\left(u_{1}\right)^{(3)},\left(u_{2}\right)^{(3)}$ :

(b) By $\left(v_{1}\right)^{(3)}>0,\left(v_{2}\right)^{(3)}<0$ and respectively $\left(u_{1}\right)^{(3)}>0,\left(u_{2}\right)^{(3)}<0$ the roots of the equations 
$\left(a_{21}\right)^{(3)}\left(v^{(3)}\right)^{2}+\left(\sigma_{1}\right)^{(3)} v^{(3)}-\left(a_{20}\right)^{(3)}=0$

and $\left(b_{21}\right)^{(3)}\left(u^{(3)}\right)^{2}+\left(\tau_{1}\right)^{(3)} u^{(3)}-\left(b_{20}\right)^{(3)}=0$ and

By $\left(\bar{v}_{1}\right)^{(3)}>0,\left(\bar{v}_{2}\right)^{(3)}<0$ and respectively $\left(\bar{u}_{1}\right)^{(3)}>0,\left(\bar{u}_{2}\right)^{(3)}<0$ the

roots of the equations $\left(a_{21}\right)^{(3)}\left(v^{(3)}\right)^{2}+\left(\sigma_{2}\right)^{(3)} v^{(3)}-\left(a_{20}\right)^{(3)}=0$

and $\left(b_{21}\right)^{(3)}\left(u^{(3)}\right)^{2}+\left(\tau_{2}\right)^{(3)} u^{(3)}-\left(b_{20}\right)^{(3)}=0$

Definition of $\left(m_{1}\right)^{(3)},\left(m_{2}\right)^{(3)},\left(\mu_{1}\right)^{(3)},\left(\mu_{2}\right)^{(3)}$ :-

(c) If we define $\left(m_{1}\right)^{(3)},\left(m_{2}\right)^{(3)},\left(\mu_{1}\right)^{(3)},\left(\mu_{2}\right)^{(3)}$ by

$$
\begin{aligned}
& \left(m_{2}\right)^{(3)}=\left(v_{0}\right)^{(3)},\left(m_{1}\right)^{(3)}=\left(v_{1}\right)^{(3)}, \text { if }\left(v_{0}\right)^{(3)}<\left(v_{1}\right)^{(3)} \\
& \left(m_{2}\right)^{(3)}=\left(v_{1}\right)^{(3)},\left(m_{1}\right)^{(3)}=\left(\bar{v}_{1}\right)^{(3)}, \text { if }\left(v_{1}\right)^{(3)}<\left(v_{0}\right)^{(3)}<\left(\bar{v}_{1}\right)^{(3)}, \\
& \text { and }\left(v_{0}\right)^{(3)}=\frac{G_{20}^{0}}{G_{21}^{0}} \\
& \left(m_{2}\right)^{(3)}=\left(v_{1}\right)^{(3)},\left(m_{1}\right)^{(3)}=\left(v_{0}\right)^{(3)}, \text { if }\left(\bar{v}_{1}\right)^{(3)}<\left(v_{0}\right)^{(3)}
\end{aligned}
$$

and analogously

$$
\begin{aligned}
& \left(\mu_{2}\right)^{(3)}=\left(u_{0}\right)^{(3)},\left(\mu_{1}\right)^{(3)}=\left(u_{1}\right)^{(3)} \text {, if }\left(u_{0}\right)^{(3)}<\left(u_{1}\right)^{(3)} \\
& \left(\mu_{2}\right)^{(3)}=\left(u_{1}\right)^{(3)},\left(\mu_{1}\right)^{(3)}=\left(\bar{u}_{1}\right)^{(3)}, \text { if }\left(u_{1}\right)^{(3)}<\left(u_{0}\right)^{(3)}<\left(\bar{u}_{1}\right)^{(3)}, \text { and }\left(u_{0}\right)^{(3)}=\frac{T_{20}^{0}}{T_{21}^{0}} \\
& \left(\mu_{2}\right)^{(3)}=\left(u_{1}\right)^{(3)},\left(\mu_{1}\right)^{(3)}=\left(u_{0}\right)^{(3)} \text {, if }\left(\bar{u}_{1}\right)^{(3)}<\left(u_{0}\right)^{(3)}
\end{aligned}
$$

Then the solution satisfies the inequalities

$$
G_{20}^{0} e^{\left(\left(S_{1}\right)^{(3)}-\left(p_{20}\right)^{(3)}\right) t} \leq G_{20}(t) \leq G_{20}^{0} e^{\left(S_{1}\right)^{(3)} t}
$$

$\left(p_{i}\right)^{(3)}$ is defined by equation above

$$
\begin{aligned}
& \frac{1}{\left(m_{1}\right)^{(3)}} G_{20}^{0} e^{\left(\left(S_{1}\right)^{(3)}-\left(p_{20}\right)^{(3)}\right) t} \leq G_{21}(t) \leq \frac{1}{\left(m_{2}\right)^{(3)}} G_{20}^{0} e^{\left(S_{1}\right)^{(3)} t} \\
& \left(\frac{\left(a_{22}\right)^{(3)} G_{20}^{0}}{\left(m_{1}\right)^{(3)}\left(\left(S_{1}\right)^{(3)}-\left(p_{20}\right)^{(3)}-\left(S_{2}\right)^{(3)}\right)}\left[e^{\left(\left(S_{1}\right)^{(3)}-\left(p_{20}\right)^{(3)}\right) t}-e^{-\left(S_{2}\right)^{(3)} t}\right]+G_{22}^{0} e^{-\left(S_{2}\right)^{(3)} t} \leq G_{22}(t) \leq\right. \\
& \left.\frac{\left(a_{22}\right)^{(3)} G_{20}^{0}}{\left(m_{2}\right)^{(3)}\left(\left(S_{1}\right)^{(3)}-\left(a_{22}^{\prime}\right)^{(3)}\right)}\left[e^{\left(S_{1}\right)^{(3)} t}-e^{-\left(a_{22}^{\prime}\right)^{(3)} t}\right]+G_{22}^{0} e^{-\left(a_{22}^{\prime}\right)^{(3)} t}\right)
\end{aligned}
$$$$
T_{20}^{0} e^{\left(R_{1}\right)^{(3)} t} \leq T_{20}(t) \leq T_{20}^{0} e^{\left(\left(R_{1}\right)^{(3)}+\left(r_{20}\right)^{(3)}\right) t}
$$

$\frac{1}{\left(\mu_{1}\right)^{(3)}} T_{20}^{0} e^{\left(R_{1}\right)^{(3)} t} \leq T_{20}(t) \leq \frac{1}{\left(\mu_{2}\right)^{(3)}} T_{20}^{0} e^{\left(\left(R_{1}\right)^{(3)}+\left(r_{20}\right)^{(3)}\right) t}$

$\frac{\left(b_{22}\right)^{(3)} T_{20}^{0}}{\left(\mu_{1}\right)^{(3)}\left(\left(R_{1}\right)^{(3)}-\left(b_{22}^{\prime}\right)^{(3)}\right)}\left[e^{\left(R_{1}\right)^{(3)} t}-e^{-\left(b_{22}^{\prime}\right)^{(3)} t}\right]+T_{22}^{0} e^{-\left(b_{22}^{\prime}\right)^{(3)} t} \leq T_{22}(t) \leq$

$\frac{\left(a_{22}\right)^{(3)} T_{20}^{0}}{\left(\mu_{2}\right)^{(3)}\left(\left(R_{1}\right)^{(3)}+\left(r_{20}\right)^{(3)}+\left(R_{2}\right)^{(3)}\right)}\left[e^{\left(\left(R_{1}\right)^{(3)}+\left(r_{20}\right)^{(3)}\right) t}-e^{-\left(R_{2}\right)^{(3)} t}\right]+T_{22}^{0} e^{-\left(R_{2}\right)^{(3)} t}$ 
Definition of $\left(S_{1}\right)^{(3)},\left(S_{2}\right)^{(3)},\left(R_{1}\right)^{(3)},\left(R_{2}\right)^{(3)}$ :-

Where $\left(S_{1}\right)^{(3)}=\left(a_{20}\right)^{(3)}\left(m_{2}\right)^{(3)}-\left(a_{20}^{\prime}\right)^{(3)}$

$$
\begin{aligned}
& \left(S_{2}\right)^{(3)}=\left(a_{22}\right)^{(3)}-\left(p_{22}\right)^{(3)} \\
& \left(R_{1}\right)^{(3)}=\left(b_{20}\right)^{(3)}\left(\mu_{2}\right)^{(3)}-\left(b_{20}^{\prime}\right)^{(3)} \\
& \left(R_{2}\right)^{(3)}=\left(b_{22}^{\prime}\right)^{(3)}-\left(r_{22}\right)^{(3)}
\end{aligned}
$$

\section{Behavior of the solutions}

If we denote and define

Definition of $\left(\sigma_{1}\right)^{(4)},\left(\sigma_{2}\right)^{(4)},\left(\tau_{1}\right)^{(4)},\left(\tau_{2}\right)^{(4)}$ :

(d) $\left(\sigma_{1}\right)^{(4)},\left(\sigma_{2}\right)^{(4)},\left(\tau_{1}\right)^{(4)},\left(\tau_{2}\right)^{(4)}$ four constants satisfying

$$
\begin{aligned}
& -\left(\sigma_{2}\right)^{(4)} \leq-\left(a_{24}^{\prime}\right)^{(4)}+\left(a_{25}^{\prime}\right)^{(4)}-\left(a_{24}^{\prime \prime}\right)^{(4)}\left(T_{25}, t\right)+\left(a_{25}^{\prime \prime}\right)^{(4)}\left(T_{25}, t\right) \leq-\left(\sigma_{1}\right)^{(4)} \\
& -\left(\tau_{2}\right)^{(4)} \leq-\left(b_{24}^{\prime}\right)^{(4)}+\left(b_{25}^{\prime}\right)^{(4)}-\left(b_{24}^{\prime \prime}\right)^{(4)}\left(\left(G_{27}\right), t\right)-\left(b_{25}^{\prime \prime}\right)^{(4)}\left(\left(G_{27}\right), t\right) \leq-\left(\tau_{1}\right)^{(4)}
\end{aligned}
$$

Definition of $\left(v_{1}\right)^{(4)},\left(v_{2}\right)^{(4)},\left(u_{1}\right)^{(4)},\left(u_{2}\right)^{(4)}, v^{(4)}, u^{(4)}$ :

(e) By $\left(v_{1}\right)^{(4)}>0,\left(v_{2}\right)^{(4)}<0$ and respectively $\left(u_{1}\right)^{(4)}>0,\left(u_{2}\right)^{(4)}<0$ the roots of the equations $\left(a_{25}\right)^{(4)}\left(v^{(4)}\right)^{2}+\left(\sigma_{1}\right)^{(4)} v^{(4)}-\left(a_{24}\right)^{(4)}=0$ and $\left(b_{25}\right)^{(4)}\left(u^{(4)}\right)^{2}+\left(\tau_{1}\right)^{(4)} u^{(4)}-\left(b_{24}\right)^{(4)}=0$ and

Definition of $\left(\bar{v}_{1}\right)^{(4)},,\left(\bar{v}_{2}\right)^{(4)},\left(\bar{u}_{1}\right)^{(4)},\left(\bar{u}_{2}\right)^{(4)}$ :

By $\left(\bar{v}_{1}\right)^{(4)}>0,\left(\bar{v}_{2}\right)^{(4)}<0$ and respectively $\left(\bar{u}_{1}\right)^{(4)}>0,\left(\bar{u}_{2}\right)^{(4)}<0$ the

roots of the equations $\left(a_{25}\right)^{(4)}\left(v^{(4)}\right)^{2}+\left(\sigma_{2}\right)^{(4)} v^{(4)}-\left(a_{24}\right)^{(4)}=0$

and $\left(b_{25}\right)^{(4)}\left(u^{(4)}\right)^{2}+\left(\tau_{2}\right)^{(4)} u^{(4)}-\left(b_{24}\right)^{(4)}=0$

Definition of $\left(m_{1}\right)^{(4)},\left(m_{2}\right)^{(4)},\left(\mu_{1}\right)^{(4)},\left(\mu_{2}\right)^{(4)},\left(v_{0}\right)^{(4)}$ :-

(f) If we define $\left(m_{1}\right)^{(4)},\left(m_{2}\right)^{(4)},\left(\mu_{1}\right)^{(4)},\left(\mu_{2}\right)^{(4)}$ by

$$
\begin{aligned}
& \left(m_{2}\right)^{(4)}=\left(v_{0}\right)^{(4)},\left(m_{1}\right)^{(4)}=\left(v_{1}\right)^{(4)}, \text { if }\left(v_{0}\right)^{(4)}<\left(v_{1}\right)^{(4)} \\
& \quad\left(m_{2}\right)^{(4)}=\left(v_{1}\right)^{(4)},\left(m_{1}\right)^{(4)}=\left(\bar{v}_{1}\right)^{(4)}, \text { if }\left(v_{4}\right)^{(4)}<\left(v_{0}\right)^{(4)}<\left(\bar{v}_{1}\right)^{(4)}, \\
& \text { and }\left(v_{0}\right)^{(4)}=\frac{G_{24}^{0}}{G_{25}^{0}} \\
& \left(m_{2}\right)^{(4)}=\left(v_{4}\right)^{(4)},\left(m_{1}\right)^{(4)}=\left(v_{0}\right)^{(4)}, \text { if }\left(\bar{v}_{4}\right)^{(4)}<\left(v_{0}\right)^{(4)}
\end{aligned}
$$

and analogously

$$
\begin{aligned}
& \left(\mu_{2}\right)^{(4)}=\left(u_{0}\right)^{(4)},\left(\mu_{1}\right)^{(4)}=\left(u_{1}\right)^{(4)}, \text { if }\left(u_{0}\right)^{(4)}<\left(u_{1}\right)^{(4)} \\
& \left(\mu_{2}\right)^{(4)}=\left(u_{1}\right)^{(4)},\left(\mu_{1}\right)^{(4)}=\left(\bar{u}_{1}\right)^{(4)}, \text { if }\left(u_{1}\right)^{(4)}<\left(u_{0}\right)^{(4)}<\left(\bar{u}_{1}\right)^{(4)}, \\
& \text { and }\left(u_{0}\right)^{(4)}=\frac{T_{24}^{0}}{T_{25}^{0}} \\
& \left(\mu_{2}\right)^{(4)}=\left(u_{1}\right)^{(4)},\left(\mu_{1}\right)^{(4)}=\left(u_{0}\right)^{(4)}, \text { if }\left(\bar{u}_{1}\right)^{(4)}<\left(u_{0}\right)^{(4)} \text { where }\left(u_{1}\right)^{(4)},\left(\bar{u}_{1}\right)^{(4)}
\end{aligned}
$$


are defined

Then the solution satisfies the inequalities

$$
G_{24}^{0} e^{\left(\left(S_{1}\right)^{(4)}-\left(p_{24}\right)^{(4)}\right) t} \leq G_{24}(t) \leq G_{24}^{0} e^{\left(S_{1}\right)^{(4)} t}
$$

where $\left(p_{i}\right)^{(4)}$ is defined by equation above

$$
\begin{aligned}
& \frac{1}{\left(m_{1}\right)^{(4)}} G_{24}^{0} e^{\left(\left(S_{1}\right)^{(4)}-\left(p_{24}\right)^{(4)}\right) t} \leq G_{25}(t) \leq \frac{1}{\left(m_{2}\right)^{(4)}} G_{24}^{0} e^{\left(S_{1}\right)^{(4)} t} \\
& \left(\frac{\left(a_{26}\right)^{(4)} G_{24}^{0}}{\left(m_{1}\right)^{(4)}\left(\left(S_{1}\right)^{(4)}-\left(p_{24}\right)^{(4)}-\left(S_{2}\right)^{(4)}\right)}\left[e^{\left(\left(S_{1}\right)^{(4)}-\left(p_{24}\right)^{(4)}\right) t}-e^{-\left(S_{2}\right)^{(4)} t}\right]+G_{26}^{0} e^{-\left(S_{2}\right)^{(4)} t} \leq G_{26}(t) \leq\right. \\
& (a 26) 4 G 240(m 2) 4(S 1) 4-\left(a 26^{\prime}\right) 4 e(S 1) 4 t-e-\left(a 26^{\prime}\right) 4 t+G 260 e-\left(a 26^{\prime}\right) 4 t
\end{aligned}
$$

$T_{24}^{0} e^{\left(R_{1}\right)^{(4)} t} \leq T_{24}(t) \leq T_{24}^{0} e^{\left(\left(R_{1}\right)^{(4)}+\left(r_{24}\right)^{(4)}\right) t}$

$\frac{1}{\left(\mu_{1}\right)^{(4)}} T_{24}^{0} e^{\left(R_{1}\right)^{(4)} t} \leq T_{24}(t) \leq \frac{1}{\left(\mu_{2}\right)^{(4)}} T_{24}^{0} e^{\left(\left(R_{1}\right)^{(4)}+\left(r_{24}\right)^{(4)}\right) t}$

$\frac{\left(b_{26}\right)^{(4)} T_{24}^{0}}{\left(\mu_{1}\right)^{(4)}\left(\left(R_{1}\right)^{(4)}-\left(b_{26}^{\prime}\right)^{(4)}\right)}\left[e^{\left(R_{1}\right)^{(4)} t}-e^{-\left(b_{26}^{\prime}\right)^{(4)} t}\right]+T_{26}^{0} e^{-\left(b_{26}^{\prime}\right)^{(4)} t} \leq T_{26}(t) \leq$

$\frac{\left(a_{26}\right)^{(4)} T_{24}^{0}}{\left(\mu_{2}\right)^{(4)}\left(\left(R_{1}\right)^{(4)}+\left(r_{24}\right)^{(4)}+\left(R_{2}\right)^{(4)}\right)}\left[e^{\left(\left(R_{1}\right)^{(4)}+\left(r_{24}\right)^{(4)}\right) t}-e^{-\left(R_{2}\right)^{(4)} t}\right]+T_{26}^{0} e^{-\left(R_{2}\right)^{(4)} t}$

Definition of $\left(S_{1}\right)^{(4)},\left(S_{2}\right)^{(4)},\left(R_{1}\right)^{(4)},\left(R_{2}\right)^{(4)}$ :-

Where $\left(S_{1}\right)^{(4)}=\left(a_{24}\right)^{(4)}\left(m_{2}\right)^{(4)}-\left(a_{24}^{\prime}\right)^{(4)}$

$$
\begin{aligned}
& \left(S_{2}\right)^{(4)}=\left(a_{26}\right)^{(4)}-\left(p_{26}\right)^{(4)} \\
& \left(R_{1}\right)^{(4)}=\left(b_{24}\right)^{(4)}\left(\mu_{2}\right)^{(4)}-\left(b_{24}^{\prime}\right)^{(4)} \\
& \left(R_{2}\right)^{(4)}=\left(b_{26}^{\prime}\right)^{(4)}-\left(r_{26}\right)^{(4)}
\end{aligned}
$$

\section{Behavior of the solutions}

If we denote and define

$$
\text { Definition of }\left(\sigma_{1}\right)^{(5)},\left(\sigma_{2}\right)^{(5)},\left(\tau_{1}\right)^{(5)},\left(\tau_{2}\right)^{(5)} \text { : }
$$

(g) $\left(\sigma_{1}\right)^{(5)},\left(\sigma_{2}\right)^{(5)},\left(\tau_{1}\right)^{(5)},\left(\tau_{2}\right)^{(5)}$ four constants satisfying

$$
\begin{aligned}
& -\left(\sigma_{2}\right)^{(5)} \leq-\left(a_{28}^{\prime}\right)^{(5)}+\left(a_{29}^{\prime}\right)^{(5)}-\left(a_{28}^{\prime \prime}\right)^{(5)}\left(T_{29}, t\right)+\left(a_{29}^{\prime \prime}\right)^{(5)}\left(T_{29}, t\right) \leq-\left(\sigma_{1}\right)^{(5)} \\
& -\left(\tau_{2}\right)^{(5)} \leq-\left(b_{28}^{\prime}\right)^{(5)}+\left(b_{29}^{\prime}\right)^{(5)}-\left(b_{28}^{\prime \prime}\right)^{(5)}\left(\left(G_{31}\right), t\right)-\left(b_{29}^{\prime \prime}\right)^{(5)}\left(\left(G_{31}\right), t\right) \leq-\left(\tau_{1}\right)^{(5)}
\end{aligned}
$$

Definition of $\left(v_{1}\right)^{(5)},\left(v_{2}\right)^{(5)},\left(u_{1}\right)^{(5)},\left(u_{2}\right)^{(5)}, v^{(5)}, u^{(5)}$ :

(h) By $\left(v_{1}\right)^{(5)}>0,\left(v_{2}\right)^{(5)}<0$ and respectively $\left(u_{1}\right)^{(5)}>0,\left(u_{2}\right)^{(5)}<0$ the roots of the equations $\left(a_{29}\right)^{(5)}\left(v^{(5)}\right)^{2}+\left(\sigma_{1}\right)^{(5)} v^{(5)}-\left(a_{28}\right)^{(5)}=0$ and $\left(b_{29}\right)^{(5)}\left(u^{(5)}\right)^{2}+\left(\tau_{1}\right)^{(5)} u^{(5)}-\left(b_{28}\right)^{(5)}=0$ and 
Definition of $\left(\bar{v}_{1}\right)^{(5)},\left(\bar{v}_{2}\right)^{(5)},\left(\bar{u}_{1}\right)^{(5)},\left(\bar{u}_{2}\right)^{(5)}$ :

By $\left(\bar{v}_{1}\right)^{(5)}>0,\left(\bar{v}_{2}\right)^{(5)}<0$ and respectively $\left(\bar{u}_{1}\right)^{(5)}>0,\left(\bar{u}_{2}\right)^{(5)}<0$ the

roots of the equations $\left(a_{29}\right)^{(5)}\left(v^{(5)}\right)^{2}+\left(\sigma_{2}\right)^{(5)} v^{(5)}-\left(a_{28}\right)^{(5)}=0$

and $\left(b_{29}\right)^{(5)}\left(u^{(5)}\right)^{2}+\left(\tau_{2}\right)^{(5)} u^{(5)}-\left(b_{28}\right)^{(5)}=0$

Definition of $\left(m_{1}\right)^{(5)},\left(m_{2}\right)^{(5)},\left(\mu_{1}\right)^{(5)},\left(\mu_{2}\right)^{(5)},\left(v_{0}\right)^{(5)}$ :-

(i) If we define $\left(m_{1}\right)^{(5)},\left(m_{2}\right)^{(5)},\left(\mu_{1}\right)^{(5)},\left(\mu_{2}\right)^{(5)}$ by

$$
\begin{aligned}
& \left(m_{2}\right)^{(5)}=\left(v_{0}\right)^{(5)},\left(m_{1}\right)^{(5)}=\left(v_{1}\right)^{(5)}, \text { if }\left(v_{0}\right)^{(5)}<\left(v_{1}\right)^{(5)} \\
& \quad\left(m_{2}\right)^{(5)}=\left(v_{1}\right)^{(5)},\left(m_{1}\right)^{(5)}=\left(\bar{v}_{1}\right)^{(5)}, \text { if }\left(v_{1}\right)^{(5)}<\left(v_{0}\right)^{(5)}<\left(\bar{v}_{1}\right)^{(5)}, \\
& \text { and }\left(v_{0}\right)^{(5)}=\frac{G_{28}^{0}}{G_{29}^{0}} \\
& \left(m_{2}\right)^{(5)}=\left(v_{1}\right)^{(5)},\left(m_{1}\right)^{(5)}=\left(v_{0}\right)^{(5)}, \text { if }\left(\bar{v}_{1}\right)^{(5)}<\left(v_{0}\right)^{(5)}
\end{aligned}
$$

and analogously

$$
\begin{aligned}
& \left(\mu_{2}\right)^{(5)}=\left(u_{0}\right)^{(5)},\left(\mu_{1}\right)^{(5)}=\left(u_{1}\right)^{(5)}, \text { if }\left(u_{0}\right)^{(5)}<\left(u_{1}\right)^{(5)} \\
& \left(\mu_{2}\right)^{(5)}=\left(u_{1}\right)^{(5)},\left(\mu_{1}\right)^{(5)}=\left(\bar{u}_{1}\right)^{(5)}, \text { if }\left(u_{1}\right)^{(5)}<\left(u_{0}\right)^{(5)}<\left(\bar{u}_{1}\right)^{(5)}, \\
& \text { and }\left(u_{0}\right)^{(5)}=\frac{T_{28}^{0}}{T_{29}^{0}}
\end{aligned}
$$

$\left(\mu_{2}\right)^{(5)}=\left(u_{1}\right)^{(5)},\left(\mu_{1}\right)^{(5)}=\left(u_{0}\right)^{(5)}, \boldsymbol{i f}\left(\bar{u}_{1}\right)^{(5)}<\left(u_{0}\right)^{(5)}$ where $\left(u_{1}\right)^{(5)},\left(\bar{u}_{1}\right)^{(5)}$

are defined respectively

Then the solution satisfies the inequalities

$G_{28}^{0} e^{\left(\left(S_{1}\right)^{(5)}-\left(p_{28}\right)^{(5)}\right) t} \leq G_{28}(t) \leq G_{28}^{0} e^{\left(S_{1}\right)^{(5)} t}$

where $\left(p_{i}\right)^{(5)}$ is defined by equation above

$$
\begin{aligned}
& \frac{1}{\left(m_{5}\right)^{(5)}} G_{28}^{0} e^{\left(\left(S_{1}\right)^{(5)}-\left(p_{28}\right)^{(5)}\right) t} \leq G_{29}(t) \leq \frac{1}{\left(m_{2}\right)^{(5)}} G_{28}^{0} e^{\left(S_{1}\right)^{(5)} t} \\
& \left(\frac{\left.a_{30}\right)^{(5)} G_{28}^{0}}{\left(m_{1}\right)^{(5)}\left(\left(S_{1}\right)^{(5)}-\left(p_{28}\right)^{(5)}-\left(S_{2}\right)^{(5)}\right)}\left[e^{\left(\left(S_{1}\right)^{(5)}-\left(p_{28}\right)^{(5)}\right) t}-e^{-\left(S_{2}\right)^{(5)} t}\right]+G_{30}^{0} e^{-\left(S_{2}\right)^{(5)} t} \leq G_{30}(t) \leq\right. \\
& (a 30) 5 G 280(m 2) 5(S 1) 5-\left(a 30^{\prime}\right) 5 e(S 1) 5 t-e-\left(a 30^{\prime}\right) 5 t+G 300 e-\left(a 30^{\prime}\right) 5 t
\end{aligned}
$$

$T_{28}^{0} e^{\left(R_{1}\right)^{(5)} t} \leq T_{28}(t) \leq T_{28}^{0} e^{\left(\left(R_{1}\right)^{(5)}+\left(r_{28}\right)^{(5)}\right) t}$

$\frac{1}{\left(\mu_{1}\right)^{(5)}} T_{28}^{0} e^{\left(R_{1}\right)^{(5)} t} \leq T_{28}(t) \leq \frac{1}{\left(\mu_{2}\right)^{(5)}} T_{28}^{0} e^{\left(\left(R_{1}\right)^{(5)}+\left(r_{28}\right)^{(5)}\right) t}$

$\frac{\left(b_{30}\right)^{(5)} T_{28}^{0}}{\left(\mu_{1}\right)^{(5)}\left(\left(R_{1}\right)^{(5)}-\left(b_{30}^{\prime}\right)^{(5)}\right)}\left[e^{\left(R_{1}\right)^{(5)} t}-e^{-\left(b_{30}^{\prime}\right)^{(5)} t}\right]+T_{30}^{0} e^{-\left(b_{30}^{\prime}\right)^{(5)} t} \leq T_{30}(t) \leq$

$\frac{\left(a_{30}\right)^{(5)} T_{28}^{0}}{\left(\mu_{2}\right)^{(5)}\left(\left(R_{1}\right)^{(5)}+\left(r_{28}\right)^{(5)}+\left(R_{2}\right)^{(5)}\right)}\left[e^{\left(\left(R_{1}\right)^{(5)}+\left(r_{28}\right)^{(5)}\right) t}-e^{-\left(R_{2}\right)^{(5)} t}\right]+T_{30}^{0} e^{-\left(R_{2}\right)^{(5)} t}$

Definition of $\left(S_{1}\right)^{(5)},\left(S_{2}\right)^{(5)},\left(R_{1}\right)^{(5)},\left(R_{2}\right)^{(5)}$ :- 
Where $\left(S_{1}\right)^{(5)}=\left(a_{28}\right)^{(5)}\left(m_{2}\right)^{(5)}-\left(a_{28}^{\prime}\right)^{(5)}$

$$
\begin{aligned}
& \left(S_{2}\right)^{(5)}=\left(a_{30}\right)^{(5)}-\left(p_{30}\right)^{(5)} \\
& \left(R_{1}\right)^{(5)}=\left(b_{28}\right)^{(5)}\left(\mu_{2}\right)^{(5)}-\left(b_{28}^{\prime}\right)^{(5)} \\
& \left(R_{2}\right)^{(5)}=\left(b_{30}^{\prime}\right)^{(5)}-\left(r_{30}\right)^{(5)}
\end{aligned}
$$

\section{Behavior of the solutions}

If we denote and define

Definition of $\left(\sigma_{1}\right)^{(6)},\left(\sigma_{2}\right)^{(6)},\left(\tau_{1}\right)^{(6)},\left(\tau_{2}\right)^{(6)}$ :

(j) $\left(\sigma_{1}\right)^{(6)},\left(\sigma_{2}\right)^{(6)},\left(\tau_{1}\right)^{(6)},\left(\tau_{2}\right)^{(6)}$ four constants satisfying

$$
\begin{aligned}
& -\left(\sigma_{2}\right)^{(6)} \leq-\left(a_{32}^{\prime}\right)^{(6)}+\left(a_{33}^{\prime}\right)^{(6)}-\left(a_{32}^{\prime \prime}\right)^{(6)}\left(T_{33}, t\right)+\left(a_{33}^{\prime \prime}\right)^{(6)}\left(T_{33}, t\right) \leq-\left(\sigma_{1}\right)^{(6)} \\
& -\left(\tau_{2}\right)^{(6)} \leq-\left(b_{32}^{\prime}\right)^{(6)}+\left(b_{33}^{\prime}\right)^{(6)}-\left(b_{32}^{\prime \prime}\right)^{(6)}\left(\left(G_{35}\right), t\right)-\left(b_{33}^{\prime \prime}\right)^{(6)}\left(\left(G_{35}\right), t\right) \leq-\left(\tau_{1}\right)^{(6)}
\end{aligned}
$$

Definition of $\left(v_{1}\right)^{(6)},\left(v_{2}\right)^{(6)},\left(u_{1}\right)^{(6)},\left(u_{2}\right)^{(6)}, v^{(6)}, u^{(6)}$ :

(k) By $\left(v_{1}\right)^{(6)}>0,\left(v_{2}\right)^{(6)}<0$ and respectively $\left(u_{1}\right)^{(6)}>0,\left(u_{2}\right)^{(6)}<0$ the roots of the equations $\left(a_{33}\right)^{(6)}\left(v^{(6)}\right)^{2}+\left(\sigma_{1}\right)^{(6)} v^{(6)}-\left(a_{32}\right)^{(6)}=0$

and $\left(b_{33}\right)^{(6)}\left(u^{(6)}\right)^{2}+\left(\tau_{1}\right)^{(6)} u^{(6)}-\left(b_{32}\right)^{(6)}=0$ and

Definition of $\left(\bar{v}_{1}\right)^{(6)},,\left(\bar{v}_{2}\right)^{(6)},\left(\bar{u}_{1}\right)^{(6)},\left(\bar{u}_{2}\right)^{(6)}$ :

By $\left(\bar{v}_{1}\right)^{(6)}>0,\left(\bar{v}_{2}\right)^{(6)}<0$ and respectively $\left(\bar{u}_{1}\right)^{(6)}>0,\left(\bar{u}_{2}\right)^{(6)}<0$ the

roots of the equations $\left(a_{33}\right)^{(6)}\left(v^{(6)}\right)^{2}+\left(\sigma_{2}\right)^{(6)} v^{(6)}-\left(a_{32}\right)^{(6)}=0$

and $\left(b_{33}\right)^{(6)}\left(u^{(6)}\right)^{2}+\left(\tau_{2}\right)^{(6)} u^{(6)}-\left(b_{32}\right)^{(6)}=0$

Definition of $\left(m_{1}\right)^{(6)},\left(m_{2}\right)^{(6)},\left(\mu_{1}\right)^{(6)},\left(\mu_{2}\right)^{(6)},\left(v_{0}\right)^{(6)}$ :-

(l) If we define $\left(m_{1}\right)^{(6)},\left(m_{2}\right)^{(6)},\left(\mu_{1}\right)^{(6)},\left(\mu_{2}\right)^{(6)}$ by

$$
\begin{aligned}
& \left(m_{2}\right)^{(6)}=\left(v_{0}\right)^{(6)},\left(m_{1}\right)^{(6)}=\left(v_{1}\right)^{(6)}, \text { if }\left(v_{0}\right)^{(6)}<\left(v_{1}\right)^{(6)} \\
& \left(m_{2}\right)^{(6)}=\left(v_{1}\right)^{(6)},\left(m_{1}\right)^{(6)}=\left(\bar{v}_{6}\right)^{(6)}, \text { if }\left(v_{1}\right)^{(6)}<\left(v_{0}\right)^{(6)}<\left(\bar{v}_{1}\right)^{(6)}, \\
& \text { and }\left(v_{0}\right)^{(6)}=\frac{G_{32}^{0}}{G_{33}^{0}} \\
& \left(m_{2}\right)^{(6)}=\left(v_{1}\right)^{(6)},\left(m_{1}\right)^{(6)}=\left(v_{0}\right)^{(6)}, \text { if }\left(\bar{v}_{1}\right)^{(6)}<\left(v_{0}\right)^{(6)}
\end{aligned}
$$

and analogously

$$
\begin{aligned}
& \left(\mu_{2}\right)^{(6)}=\left(u_{0}\right)^{(6)},\left(\mu_{1}\right)^{(6)}=\left(u_{1}\right)^{(6)}, \text { if }\left(u_{0}\right)^{(6)}<\left(u_{1}\right)^{(6)} \\
& \left(\mu_{2}\right)^{(6)}=\left(u_{1}\right)^{(6)},\left(\mu_{1}\right)^{(6)}=\left(\bar{u}_{1}\right)^{(6)}, \text { if }\left(u_{1}\right)^{(6)}<\left(u_{0}\right)^{(6)}<\left(\bar{u}_{1}\right)^{(6)}, \\
& \text { and }\left(u_{0}\right)^{(6)}=\frac{T_{32}^{0}}{T_{33}^{0}} \\
& \left(\mu_{2}\right)^{(6)}=\left(u_{1}\right)^{(6)},\left(\mu_{1}\right)^{(6)}=\left(u_{0}\right)^{(6)}, \text { if }\left(\bar{u}_{1}\right)^{(6)}<\left(u_{0}\right)^{(6)} \text { where }\left(u_{1}\right)^{(6)},\left(\bar{u}_{1}\right)^{(6)}
\end{aligned}
$$
are defined respectively 
Then the solution satisfies the inequalities

$$
G_{32}^{0} e^{\left(\left(S_{1}\right)^{(6)}-\left(p_{32}\right)^{(6)}\right) t} \leq G_{32}(t) \leq G_{32}^{0} e^{\left(S_{1}\right)^{(6)} t}
$$

where $\left(p_{i}\right)^{(6)}$ is defined by equation above

$$
\begin{aligned}
& \frac{1}{\left(m_{1}\right)^{(6)}} G_{32}^{0} e^{\left(\left(S_{1}\right)^{(6)}-\left(p_{32}\right)^{(6)}\right) t} \leq G_{33}(t) \leq \frac{1}{\left(m_{2}\right)^{(6)}} G_{32}^{0} e^{\left(S_{1}\right)^{(6)} t} \\
& \left(\frac{\left(a_{34}\right)^{(6)} G_{32}^{0}}{\left(m_{1}\right)^{(6)}\left(\left(S_{1}\right)^{(6)}-\left(p_{32}\right)^{(6)}-\left(S_{2}\right)^{(6)}\right)}\left[e^{\left(\left(S_{1}\right)^{(6)}-\left(p_{32}\right)^{(6)}\right) t}-e^{-\left(S_{2}\right)^{(6)} t}\right]+G_{34}^{0} e^{-\left(S_{2}\right)^{(6)} t} \leq G_{34}(t) \leq\right.
\end{aligned}
$$

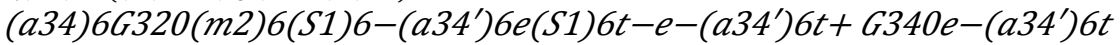

$T_{32}^{0} e^{\left(R_{1}\right)^{(6)} t} \leq T_{32}(t) \leq T_{32}^{0} e^{\left(\left(R_{1}\right)^{(6)}+\left(r_{32}\right)^{(6)}\right) t}$

$\frac{1}{\left(\mu_{1}\right)^{(6)}} T_{32}^{0} e^{\left(R_{1}\right)^{(6)} t} \leq T_{32}(t) \leq \frac{1}{\left(\mu_{2}\right)^{(6)}} T_{32}^{0} e^{\left(\left(R_{1}\right)^{(6)}+\left(r_{32}\right)^{(6)}\right) t}$

$\frac{\left(b_{34}\right)^{(6)} T_{32}^{0}}{\left(\mu_{1}\right)^{(6)}\left(\left(R_{1}\right)^{(6)}-\left(b_{34}^{\prime}\right)^{(6)}\right)}\left[e^{\left(R_{1}\right)^{(6)} t}-e^{-\left(b_{34}^{\prime}\right)^{(6)} t}\right]+T_{34}^{0} e^{-\left(b_{34}^{\prime}\right)^{(6)} t} \leq T_{34}(t) \leq$

Definition of $\left(S_{1}\right)^{(6)},\left(S_{2}\right)^{(6)},\left(R_{1}\right)^{(6)},\left(R_{2}\right)^{(6)}:-$

Where $\left(S_{1}\right)^{(6)}=\left(a_{32}\right)^{(6)}\left(m_{2}\right)^{(6)}-\left(a_{32}^{\prime}\right)^{(6)}$

$$
\begin{aligned}
& \left(S_{2}\right)^{(6)}=\left(a_{34}\right)^{(6)}-\left(p_{34}\right)^{(6)} \\
& \left(R_{1}\right)^{(6)}=\left(b_{32}\right)^{(6)}\left(\mu_{2}\right)^{(6)}-\left(b_{32}^{\prime}\right)^{(6)} \\
& \left(R_{2}\right)^{(6)}=\left(b_{34}^{\prime}\right)^{(6)}-\left(r_{34}\right)^{(6)}
\end{aligned}
$$

Proof : From Governing equations we obtain $\frac{d v^{(1)}}{d t}=\left(a_{13}\right)^{(1)}-\left(\left(a_{13}^{\prime}\right)^{(1)}-\left(a_{14}^{\prime}\right)^{(1)}+\left(a_{13}^{\prime \prime}\right)^{(1)}\left(T_{14}, t\right)\right)-\left(a_{14}^{\prime \prime}\right)^{(1)}\left(T_{14}, t\right) v^{(1)}-\left(a_{14}\right)^{(1)} v^{(1)}$

Definition of $v^{(1)}:-\quad v^{(1)}=\frac{G_{13}}{G_{14}}$

It follows

$$
-\left(\left(a_{14}\right)^{(1)}\left(v^{(1)}\right)^{2}+\left(\sigma_{2}\right)^{(1)} v^{(1)}-\left(a_{13}\right)^{(1)}\right) \leq \frac{d v^{(1)}}{d t} \leq-\left(\left(a_{14}\right)^{(1)}\left(v^{(1)}\right)^{2}+\left(\sigma_{1}\right)^{(1)} v^{(1)}-\left(a_{13}\right)^{(1)}\right)
$$

From which one obtains

Definition of $\left(\bar{v}_{1}\right)^{(1)},\left(v_{0}\right)^{(1)}$ :-

(a) For $0<\left(v_{0}\right)^{(1)}=\frac{G_{13}^{0}}{G_{14}^{0}}<\left(v_{1}\right)^{(1)}<\left(\bar{v}_{1}\right)^{(1)}$ 


$$
\begin{gathered}
v^{(1)}(t) \geq \frac{\left(v_{1}\right)^{(1)}+(C)^{(1)}\left(v_{2}\right)^{(1)} e^{\left[-\left(a_{14}\right)^{(1)}\left(\left(_{1}\right)^{(1)}-\left(v_{0}\right)^{(1)}\right) t\right]}}{1+(C)^{(1)} e^{\left.\left.-\left(a_{14}\right)^{(1)}\left(v_{1}\right)^{(1)}-\left(v_{0}\right)^{(1)}\right) t\right]}}, \quad(C)^{(1)}=\frac{\left(v_{1}\right)^{(1)}-\left(v_{0}\right)^{(1)}}{\left(v_{0}\right)^{(1)}-\left(v_{2}\right)^{(1)}} \\
\text { it follows }\left(v_{0}\right)^{(1)} \leq v^{(1)}(t) \leq\left(v_{1}\right)^{(1)}
\end{gathered}
$$

In the same manner, we get

$$
v^{(1)}(t) \leq \frac{\left(\bar{v}_{1}\right)^{(1)}+(\bar{C})^{(1)}\left(\bar{v}_{2}\right)^{(1)} e^{\left.\left[-\left(a_{14}\right)^{(1)}\left(\bar{v}_{1}\right)^{(1)}-\left(\bar{v}_{2}\right)^{(1)}\right) t\right]}}{1+(\bar{C})^{(1)} e^{\left.\left.-\left(a_{14}\right)^{(1)}\left(\bar{v}_{1}\right)^{(1)}-\left(\bar{v}_{2}\right)^{(1)}\right) t\right]}} \quad, \quad(\bar{C})^{(1)}=\frac{\left(\bar{v}_{1}\right)^{(1)}-\left(v_{0}\right)^{(1)}}{\left(v_{0}\right)^{(1)}-\left(\bar{v}_{2}\right)^{(1)}}
$$

From which we deduce $\left(v_{0}\right)^{(1)} \leq v^{(1)}(t) \leq\left(\bar{v}_{1}\right)^{(1)}$

(b) If $0<\left(v_{1}\right)^{(1)}<\left(v_{0}\right)^{(1)}=\frac{G_{13}^{0}}{G_{14}^{0}}<\left(\bar{v}_{1}\right)^{(1)}$ we find like in the previous case,

$$
\begin{gathered}
\left(v_{1}\right)^{(1)} \leq \frac{\left(v_{1}\right)^{(1)}+(C)^{(1)}\left(v_{2}\right)^{(1)} e^{\left[-\left(a_{14}\right)^{(1)}\left(\left(_{1}\right)^{(1)}-\left(v_{2}\right)^{(1)}\right) t\right]}}{1+(C)^{(1)} e^{\left.\left.-\left(a_{14}\right)^{(1)}\left(v_{1}\right)^{(1)}-\left(v_{2}\right)^{(1)}\right) t\right]}} \leq v^{(1)}(t) \leq \\
\frac{\left(\bar{v}_{1}\right)^{(1)}+(\bar{C})^{(1)}\left(\bar{v}_{2}\right)^{(1)} e^{\left.\left[-\left(a_{14}\right)^{(1)}\left(\bar{v}_{1}\right)^{(1)}-\left(\bar{v}_{2}\right)^{(1)}\right) t\right]}}{1+(\bar{C})^{(1)} e^{\left.\left[-\left(a_{14}\right)^{(1)}\left(\bar{v}_{1}\right)^{(1)}-\left(\bar{v}_{2}\right)^{(1)}\right) t\right]}} \leq\left(\bar{v}_{1}\right)^{(1)}
\end{gathered}
$$

(c) If $0<\left(v_{1}\right)^{(1)} \leq\left(\bar{v}_{1}\right)^{(1)} \leq\left(v_{0}\right)^{(1)}=\frac{G_{13}^{0}}{G_{14}^{0}}$, we obtain

$$
\left(v_{1}\right)^{(1)} \leq v^{(1)}(t) \leq \frac{\left(\bar{v}_{1}\right)^{(1)}+(\bar{C})^{(1)}\left(\bar{\nu}_{2}\right)^{(1)} e^{\left.\left[-\left(a_{14}\right)^{(1)}\left(\bar{v}_{1}\right)^{(1)}-\left(\bar{v}_{2}\right)^{(1)}\right) t\right]}}{1+\left(\bar{C}^{(1)} e^{\left.\left[-\left(a_{14}\right)^{(1)}\left(\bar{v}_{1}\right)^{(1)}-\left(\bar{v}_{2}\right)^{(1)}\right) t\right]}\right.} \leq\left(v_{0}\right)^{(1)}
$$

And so with the notation of the first part of condition (c), we have

Definition of $v^{(1)}(t)$ :-

$$
\left(m_{2}\right)^{(1)} \leq v^{(1)}(t) \leq\left(m_{1}\right)^{(1)}, \quad v^{(1)}(t)=\frac{G_{13}(t)}{G_{14}(t)}
$$

In a completely analogous way, we obtain

Definition of $u^{(1)}(t)$ :-

$\left(\mu_{2}\right)^{(1)} \leq u^{(1)}(t) \leq\left(\mu_{1}\right)^{(1)}, \quad u^{(1)}(t)=\frac{T_{13}(t)}{T_{14}(t)}$

Now, using this result and replacing it in concatenated equations of global system we get easily the result stated in the theorem.

Particular case :

If $\left(a_{13}^{\prime \prime}\right)^{(1)}=\left(a_{14}^{\prime \prime}\right)^{(1)}$, then $\left(\sigma_{1}\right)^{(1)}=\left(\sigma_{2}\right)^{(1)}$ and in this case $\left(v_{1}\right)^{(1)}=\left(\bar{v}_{1}\right)^{(1)}$ if in addition $\left(v_{0}\right)^{(1)}=$ $\left(v_{1}\right)^{(1)}$ then $v^{(1)}(t)=\left(v_{0}\right)^{(1)}$ and as a consequence $G_{13}(t)=\left(v_{0}\right)^{(1)} G_{14}(t)$ this also defines $\left(v_{0}\right)^{(1)}$ for the special case

Analogously if $\left(b_{13}^{\prime \prime}\right)^{(1)}=\left(b_{14}^{\prime \prime}\right)^{(1)}$, then $\left(\tau_{1}\right)^{(1)}=\left(\tau_{2}\right)^{(1)}$ and then

$\left(u_{1}\right)^{(1)}=\left(\bar{u}_{1}\right)^{(1)}$ if in addition $\left(u_{0}\right)^{(1)}=\left(u_{1}\right)^{(1)}$ then $T_{13}(t)=\left(u_{0}\right)^{(1)} T_{14}(t)$ This is an important consequence of the relation between $\left(v_{1}\right)^{(1)}$ and $\left(\bar{v}_{1}\right)^{(1)}$, and definition of $\left(u_{0}\right)^{(1)}$.

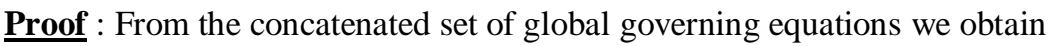


$\frac{\mathrm{d} v^{(2)}}{\mathrm{dt}}=\left(a_{16}\right)^{(2)}-\left(\left(a_{16}^{\prime}\right)^{(2)}-\left(a_{17}^{\prime}\right)^{(2)}+\left(a_{16}^{\prime \prime}\right)^{(2)}\left(\mathrm{T}_{17}, \mathrm{t}\right)\right)-\left(a_{17}^{\prime \prime}\right)^{(2)}\left(\mathrm{T}_{17}, \mathrm{t}\right) v^{(2)}-\left(a_{17}\right)^{(2)} v^{(2)}$

$\underline{\text { Definition of }} v^{(2)}:-$

$$
v^{(2)}=\frac{G_{16}}{G_{17}}
$$

It follows

$$
-\left(\left(a_{17}\right)^{(2)}\left(v^{(2)}\right)^{2}+\left(\sigma_{2}\right)^{(2)} v^{(2)}-\left(a_{16}\right)^{(2)}\right) \leq \frac{\mathrm{d} v^{(2)}}{\mathrm{dt}} \leq-\left(\left(a_{17}\right)^{(2)}\left(v^{(2)}\right)^{2}+\left(\sigma_{1}\right)^{(2)} v^{(2)}-\left(a_{16}\right)^{(2)}\right)
$$

From which one obtains

Definition of $\left(\bar{v}_{1}\right)^{(2)},\left(v_{0}\right)^{(2)}:-$

(d) For $0<\left(v_{0}\right)^{(2)}=\frac{\mathrm{G}_{16}^{0}}{\mathrm{G}_{17}^{0}}<\left(v_{1}\right)^{(2)}<\left(\bar{v}_{1}\right)^{(2)}$

$$
v^{(2)}(t) \geq \frac{\left(v_{1}\right)^{(2)}+(\mathrm{C})^{(2)}\left(v_{2}\right)^{(2)} e^{\left[-\left(a_{17}\right)^{(2)}\left(\left(v_{1}\right)^{(2)}-\left(v_{0}\right)^{(2)}\right) t\right]}}{1+(\mathrm{C})^{(2)} e^{\left.-\left(a_{17}\right)^{(2)}\left(\left(v_{1}\right)^{(2)}-\left(v_{0}\right)^{(2)}\right) t\right]}} \quad, \quad(\mathrm{C})^{(2)}=\frac{\left(v_{1}\right)^{(2)}-\left(v_{0}\right)^{(2)}}{\left(v_{0}\right)^{(2)}-\left(v_{2}\right)^{(2)}}
$$

it follows $\left(v_{0}\right)^{(2)} \leq v^{(2)}(t) \leq\left(v_{1}\right)^{(2)}$

In the same manner, we get

$$
v^{(2)}(t) \leq \frac{\left(\bar{v}_{1}\right)^{(2)}+(\bar{C})(2)\left(\bar{v}_{2}\right)^{(2)} e^{\left.\left[-\left(a_{17}\right)^{(2)}\left(\bar{v}_{1}\right)^{(2)}-\left(\bar{v}_{2}\right)^{(2)}\right) t\right]}}{1+(\overline{\mathrm{C}})^{(2)} e^{\left.\left[-\left(a_{17}\right)^{(2)}\left(\bar{v}_{1}\right)^{(2)}-\left(\bar{v}_{2}\right)^{(2)}\right) t\right]}} \quad,(\overline{\mathrm{C}})^{(2)}=\frac{\left(\bar{v}_{1}\right)^{(2)}-\left(v_{0}\right)^{(2)}}{\left(v_{0}\right)^{(2)}-\left(\bar{v}_{2}\right)^{(2)}}
$$

From which we deduce $\left(v_{0}\right)^{(2)} \leq v^{(2)}(t) \leq\left(\bar{v}_{1}\right)^{(2)}$

(e) If $0<\left(v_{1}\right)^{(2)}<\left(v_{0}\right)^{(2)}=\frac{\mathrm{G}_{16}^{0}}{\mathrm{G}_{17}^{0}}<\left(\bar{v}_{1}\right)^{(2)}$ we find like in the previous case,

$$
\begin{aligned}
& \left(v_{1}\right)^{(2)} \leq \frac{\left(v_{1}\right)^{(2)}+(\mathrm{C})^{(2)}\left(v_{2}\right)^{(2)} e^{\left[-\left(a_{17}\right)^{(2)}\left(\left(_{1}\right)^{(2)}-\left(v_{2}\right)^{(2)}\right) t\right]}}{1+(\mathrm{C})^{(2)} e^{\left.\left[-\left(a_{17}\right)^{(2)}\left(v_{1}\right)^{(2)}-\left(v_{2}\right)^{(2)}\right) t\right]}} \leq v^{(2)}(t) \leq \\
& \frac{\left(\bar{v}_{1}\right)^{(2)}+(\overline{\mathrm{C}})^{(2)}\left(\bar{v}_{2}\right)^{(2)} e^{\left.\left[-\left(a_{17}\right)^{(2)}\left(\bar{v}_{1}\right)^{(2)}-\left(\bar{v}_{2}\right)^{(2)}\right) t\right]}}{1+(\overline{\mathrm{C}})^{(2)} e^{\left.\left[-\left(a_{17}\right)^{(2)}\left(\bar{v}_{1}\right)^{(2)}-\left(\bar{v}_{2}\right)^{(2)}\right) t\right]}} \leq\left(\bar{v}_{1}\right)^{(2)}
\end{aligned}
$$

(f) If $0<\left(v_{1}\right)^{(2)} \leq\left(\bar{v}_{1}\right)^{(2)} \leq\left(v_{0}\right)^{(2)}=\frac{\mathrm{G}_{16}^{0}}{\mathrm{G}_{17}^{0}}$, we obtain

$$
\left(v_{1}\right)^{(2)} \leq v^{(2)}(t) \leq \frac{\left(\bar{v}_{1}\right)^{(2)}+(\overline{\mathcal{C}})^{(2)}\left(\bar{v}_{2}\right)^{(2)} e^{\left.\left[-\left(a_{17}\right)^{(2)}\left(\bar{v}_{1}\right)^{(2)}-\left(\bar{v}_{2}\right)^{(2)}\right) t\right]}}{1+(\bar{C})^{(2)} e^{\left.\left[-\left(a_{17}\right)^{(2)}\left(\bar{v}_{1}\right)^{(2)}-\left(\bar{v}_{2}\right)^{(2)}\right) t\right]}} \leq\left(v_{0}\right)^{(2)}
$$

And so with the notation of the first part of condition (c), we have

Definition of $v^{(2)}(t)$ :-

$\left(m_{2}\right)^{(2)} \leq v^{(2)}(t) \leq\left(m_{1}\right)^{(2)}, v^{(2)}(t)=\frac{G_{16}(t)}{G_{17}(t)}$

In a completely analogous way, we obtain

Definition of $u^{(2)}(t)$ :- 
$\left(\mu_{2}\right)^{(2)} \leq u^{(2)}(t) \leq\left(\mu_{1}\right)^{(2)}, u^{(2)}(t)=\frac{T_{16}(t)}{T_{17}(t)}$

Now, using this result and replacing it in global equations we get easily the result stated in the theorem.

\section{Particular case :}

If $\left(a_{16}^{\prime \prime}\right)^{(2)}=\left(a_{17}^{\prime \prime}\right)^{(2)}$, then $\left(\sigma_{1}\right)^{(2)}=\left(\sigma_{2}\right)^{(2)}$ and in this case $\left(v_{1}\right)^{(2)}=\left(\bar{v}_{1}\right)^{(2)}$ if in addition $\left(v_{0}\right)^{(2)}=$ $\left(v_{1}\right)^{(2)}$ then $v^{(2)}(t)=\left(v_{0}\right)^{(2)}$ and as a consequence $G_{16}(t)=\left(v_{0}\right)^{(2)} G_{17}(t)$

Analogously if $\left(b_{16}^{\prime \prime}\right)^{(2)}=\left(b_{17}^{\prime \prime}\right)^{(2)}$, then $\left(\tau_{1}\right)^{(2)}=\left(\tau_{2}\right)^{(2)}$ and then

$\left(u_{1}\right)^{(2)}=\left(\bar{u}_{1}\right)^{(2)}$ if in addition $\left(u_{0}\right)^{(2)}=\left(u_{1}\right)^{(2)}$ then $T_{16}(t)=\left(u_{0}\right)^{(2)} T_{17}(t)$ This is an important consequence of the relation between $\left(v_{1}\right)^{(2)}$ and $\left(\bar{v}_{1}\right)^{(2)}$

Proof : From Global equations we obtain

$\frac{d v^{(3)}}{d t}=\left(a_{20}\right)^{(3)}-\left(\left(a_{20}^{\prime}\right)^{(3)}-\left(a_{21}^{\prime}\right)^{(3)}+\left(a_{20}^{\prime \prime}\right)^{(3)}\left(T_{21}, t\right)\right)-\left(a_{21}^{\prime \prime}\right)^{(3)}\left(T_{21}, t\right) v^{(3)}-\left(a_{21}\right)^{(3)} v^{(3)}$

Definition of $v^{(3)}:-\quad v^{(3)}=\frac{G_{20}}{G_{21}}$

It follows

$$
-\left(\left(a_{21}\right)^{(3)}\left(v^{(3)}\right)^{2}+\left(\sigma_{2}\right)^{(3)} v^{(3)}-\left(a_{20}\right)^{(3)}\right) \leq \frac{d v^{(3)}}{d t} \leq-\left(\left(a_{21}\right)^{(3)}\left(v^{(3)}\right)^{2}+\left(\sigma_{1}\right)^{(3)} v^{(3)}-\left(a_{20}\right)^{(3)}\right)
$$

From which one obtains

(a) For $0<\left(v_{0}\right)^{(3)}=\frac{G_{20}^{0}}{G_{21}^{0}}<\left(v_{1}\right)^{(3)}<\left(\bar{v}_{1}\right)^{(3)}$

$v^{(3)}(t) \geq \frac{\left(v_{1}\right)^{(3)}+(C)^{(3)}\left(v_{2}\right)^{(3)} e^{\left[-\left(a_{21}\right)^{(3)}\left(\left(v_{1}\right)^{(3)}-\left(v_{0}\right)^{(3)}\right) t\right]}}{1+(C)^{(3)} e^{\left.\left.-\left(a_{21}\right)^{(3)}\left(v_{1}\right)^{(3)}-\left(v_{0}\right)^{(3)}\right) t\right]}} \quad, \quad(C)^{(3)}=\frac{\left(v_{1}\right)^{(3)}-\left(v_{0}\right)^{(3)}}{\left(v_{0}\right)^{(3)}-\left(v_{2}\right)^{(3)}}$

it follows $\left(v_{0}\right)^{(3)} \leq v^{(3)}(t) \leq\left(v_{1}\right)^{(3)}$

In the same manner, we get

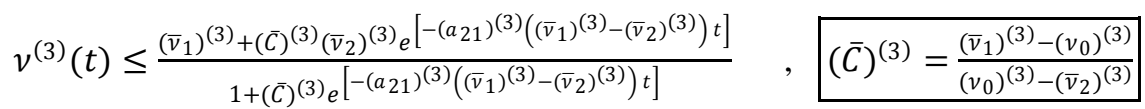

Definition of $\left(\bar{v}_{1}\right)^{(3)}$ :-

From which we deduce $\left(v_{0}\right)^{(3)} \leq v^{(3)}(t) \leq\left(\bar{v}_{1}\right)^{(3)}$

(b) If $0<\left(v_{1}\right)^{(3)}<\left(v_{0}\right)^{(3)}=\frac{G_{20}^{0}}{G_{21}^{0}}<\left(\bar{v}_{1}\right)^{(3)}$ we find like in the previous case, $\left(v_{1}\right)^{(3)} \leq \frac{\left(v_{1}\right)^{(3)}+(C)^{(3)}\left(v_{2}\right)^{(3)} e^{\left[-\left(a_{21}\right)^{(3)}\left(\left(v_{1}\right)^{(3)}-\left(v_{2}\right)^{(3)}\right) t\right]}}{1+(C)^{(3)} e^{\left.-\left(a_{21}\right)^{(3)}\left(\left(v_{1}\right)^{(3)}-\left(v_{2}\right)^{(3)}\right) t\right]}} \leq v^{(3)}(t) \leq$ $\frac{\left(\bar{v}_{1}\right)^{(3)}+(\bar{C})^{(3)}\left(\bar{v}_{2}\right)^{(3)} e^{\left.\left[-\left(a_{21}\right)^{(3)}\left(\bar{v}_{1}\right)^{(3)}-\left(\bar{v}_{2}\right)^{(3)}\right) t\right]}}{1+(\bar{C})^{(3)} e^{\left.\left.-\left(a_{21}\right)^{(3)}\left(\bar{v}_{1}\right)^{(3)}-\left(\bar{v}_{2}\right)^{(3)}\right) t\right]}} \leq\left(\bar{v}_{1}\right)^{(3)}$

(c) If $0<\left(v_{1}\right)^{(3)} \leq\left(\bar{v}_{1}\right)^{(3)} \leq\left(v_{0}\right)^{(3)}=\frac{G_{20}^{0}}{G_{21}^{0}}$, we obtain 
$\left(v_{1}\right)^{(3)} \leq v^{(3)}(t) \leq \frac{\left(\bar{v}_{1}\right)^{(3)}+(\bar{C})^{(3)}\left(\bar{v}_{2}\right)^{(3)} e^{\left[-\left(a_{21}\right)^{(3)}\left(\left(\bar{v}_{1}\right)^{(3)}-\left(\bar{v}_{2}\right)^{(3)}\right) t\right]}}{1+(\bar{C})^{(3)} e^{\left.\left.-\left(a_{21}\right)^{(3)}\left(\bar{v}_{1}\right)^{(3)}-\left(\bar{v}_{2}\right)^{(3)}\right) t\right]}} \leq\left(v_{0}\right)^{(3)}$

And so with the notation of the first part of condition (c), we have

Definition of $v^{(3)}(t)$ :-

$\left(m_{2}\right)^{(3)} \leq v^{(3)}(t) \leq\left(m_{1}\right)^{(3)}, v^{(3)}(t)=\frac{G_{20}(t)}{G_{21}(t)}$

In a completely analogous way, we obtain

Definition of $u^{(3)}(t)$ :-

$\left(\mu_{2}\right)^{(3)} \leq u^{(3)}(t) \leq\left(\mu_{1}\right)^{(3)}, u^{(3)}(t)=\frac{T_{20}(t)}{T_{21}(t)}$

Now, using this result and replacing it in Global Equations we get easily the result stated in the theorem.

Particular case :

If $\left(a_{20}^{\prime \prime}\right)^{(3)}=\left(a_{21}^{\prime \prime}\right)^{(3)}$, then $\left(\sigma_{1}\right)^{(3)}=\left(\sigma_{2}\right)^{(3)}$ and in this case $\left(v_{1}\right)^{(3)}=\left(\bar{v}_{1}\right)^{(3)}$ if in addition $\left(v_{0}\right)^{(3)}=$ $\left(v_{1}\right)^{(3)}$ then $v^{(3)}(t)=\left(v_{0}\right)^{(3)}$ and as a consequence $G_{20}(t)=\left(v_{0}\right)^{(3)} G_{21}(t)$

Analogously if $\left(b_{20}^{\prime \prime}\right)^{(3)}=\left(b_{21}^{\prime \prime}\right)^{(3)}$, then $\left(\tau_{1}\right)^{(3)}=\left(\tau_{2}\right)^{(3)}$ and then

$\left(u_{1}\right)^{(3)}=\left(\bar{u}_{1}\right)^{(3)}$ if in addition $\left(u_{0}\right)^{(3)}=\left(u_{1}\right)^{(3)}$ then $T_{20}(t)=\left(u_{0}\right)^{(3)} T_{21}(t)$ This is an important consequence of the relation between $\left(v_{1}\right)^{(3)}$ and $\left(\bar{v}_{1}\right)^{(3)}$

Proof : From Global equations we obtain

$\frac{d v^{(4)}}{d t}=\left(a_{24}\right)^{(4)}-\left(\left(a_{24}^{\prime}\right)^{(4)}-\left(a_{25}^{\prime}\right)^{(4)}+\left(a_{24}^{\prime \prime}\right)^{(4)}\left(T_{25}, t\right)\right)-\left(a_{25}^{\prime \prime}\right)^{(4)}\left(T_{25}, t\right) v^{(4)}-\left(a_{25}\right)^{(4)} v^{(4)}$

Definition of $v^{(4)}:-\quad v^{(4)}=\frac{G_{24}}{G_{25}}$

It follows

$-\left(\left(a_{25}\right)^{(4)}\left(v^{(4)}\right)^{2}+\left(\sigma_{2}\right)^{(4)} v^{(4)}-\left(a_{24}\right)^{(4)}\right) \leq \frac{d v^{(4)}}{d t} \leq-\left(\left(a_{25}\right)^{(4)}\left(v^{(4)}\right)^{2}+\left(\sigma_{4}\right)^{(4)} v^{(4)}-\left(a_{24}\right)^{(4)}\right)$

From which one obtains

Definition of $\left(\bar{v}_{1}\right)^{(4)},\left(v_{0}\right)^{(4)}$ :-

(d) For $0<\left(v_{0}\right)^{(4)}=\frac{G_{24}^{0}}{G_{25}^{0}}<\left(v_{1}\right)^{(4)}<\left(\bar{v}_{1}\right)^{(4)}$

$$
v^{(4)}(t) \geq \frac{\left(v_{1}\right)^{(4)}+(C)^{(4)}\left(v_{2}\right)^{(4)} e^{\left[-\left(a_{25}\right)^{(4)}\left(\left(v_{1}\right)^{(4)}-\left(v_{0}\right)^{(4)}\right) t\right]}}{4+(C)^{(4)} e^{\left[-\left(a_{25}\right)^{(4)}\left(\left(v_{1}\right)^{(4)}-\left(v_{0}\right)^{(4)}\right) t\right]}} \quad, \quad(C)^{(4)}=\frac{\left(v_{1}\right)^{(4)}-\left(v_{0}\right)^{(4)}}{\left(v_{0}\right)^{(4)}-\left(v_{2}\right)^{(4)}}
$$

it follows $\left(v_{0}\right)^{(4)} \leq v^{(4)}(t) \leq\left(v_{1}\right)^{(4)}$

In the same manner, we get

$$
v^{(4)}(t) \leq \frac{\left(\bar{v}_{1}\right)^{(4)}+(\bar{C})^{(4)}\left(\bar{v}_{2}\right)^{(4)} e^{\left.\left[-\left(a_{25}\right)^{(4)}\left(\overline{(v}_{1}\right)^{(4)}-\left(\bar{v}_{2}\right)^{(4)}\right) t\right]}}{4+(\bar{C})^{(4)} e^{\left.\left.-\left(a_{25}\right)^{(4)}\left(\bar{v}_{1}\right)^{(4)}-\left(\bar{v}_{2}\right)^{(4)}\right) t\right]}} \quad, \quad(\bar{C})^{(4)}=\frac{\left(\bar{v}_{1}\right)^{(4)}-\left(v_{0}\right)^{(4)}}{\left(v_{0}\right)^{(4)}-\left(\bar{v}_{2}\right)^{(4)}}
$$


From which we deduce $\left(v_{0}\right)^{(4)} \leq v^{(4)}(t) \leq\left(\bar{v}_{1}\right)^{(4)}$

(e) If $0<\left(v_{1}\right)^{(4)}<\left(v_{0}\right)^{(4)}=\frac{G_{24}^{0}}{G_{25}^{0}}<\left(\bar{v}_{1}\right)^{(4)}$ we find like in the previous case,

$$
\begin{gathered}
\left(v_{1}\right)^{(4)} \leq \frac{\left(v_{1}\right)^{(4)}+(C)^{(4)}\left(v_{2}\right)^{(4)} e^{\left[-\left(a_{25}\right)^{(4)}\left(\left(v_{1}\right)^{(4)}-\left(v_{2}\right)^{(4)}\right) t\right]}}{1+(C)^{(4)} e^{\left.\left.-\left(a_{25}\right)^{(4)}\left(v_{1}\right)^{(4)}-\left(v_{2}\right)^{(4)}\right) t\right]}} \leq v^{(4)}(t) \leq \\
\frac{\left(\bar{v}_{1}\right)^{(4)}+(\bar{C})^{(4)}\left(\bar{v}_{2}\right)^{(4)} e^{\left.\left[-\left(a_{25}\right)^{(4)}\left(\bar{v}_{1}\right)^{(4)}-\left(\bar{v}_{2}\right)^{(4)}\right) t\right]}}{1+(\bar{C})^{(4)} e^{\left.-\left(a_{25}\right)^{(4)}\left(\left(\bar{v}_{1}\right)^{(4)}-\left(\bar{v}_{2}\right)^{(4)}\right) t\right]}} \leq\left(\bar{v}_{1}\right)^{(4)}
\end{gathered}
$$

(f) If $0<\left(v_{1}\right)^{(4)} \leq\left(\bar{v}_{1}\right)^{(4)} \leq\left(v_{0}\right)^{(4)}=\frac{G_{24}^{0}}{G_{25}^{0}}$, we obtain

$$
\left(v_{1}\right)^{(4)} \leq v^{(4)}(t) \leq \frac{\left(\bar{v}_{1}\right)^{(4)}+(\bar{C})^{(4)}\left(\bar{v}_{2}\right)^{(4)} e^{\left.\left[-\left(a_{25}\right)^{(4)}\left(\bar{v}_{1}\right)^{(4)}-\left(\bar{v}_{2}\right)^{(4)}\right) t\right]}}{1+(\bar{C})^{(4)} e^{\left.\left[-\left(a_{25}\right)^{(4)}\left(\bar{v}_{1}\right)^{(4)}-\left(\bar{v}_{2}\right)^{(4)}\right) t\right]}} \leq\left(v_{0}\right)^{(4)}
$$

And so with the notation of the first part of condition (c), we have

Definition of $v^{(4)}(t)$ :-

$\left(m_{2}\right)^{(4)} \leq v^{(4)}(t) \leq\left(m_{1}\right)^{(4)}, v^{(4)}(t)=\frac{G_{24}(t)}{G_{25}(t)}$

In a completely analogous way, we obtain

Definition of $u^{(4)}(t)$ :-

$\left(\mu_{2}\right)^{(4)} \leq u^{(4)}(t) \leq\left(\mu_{1}\right)^{(4)}, u^{(4)}(t)=\frac{T_{24}(t)}{T_{25}(t)}$

Now, using this result and replacing it in Global equations we get easily the result stated in the theorem.

\section{Particular case :}

If $\left(a_{24}^{\prime \prime}\right)^{(4)}=\left(a_{25}^{\prime \prime}\right)^{(4)}$, then $\left(\sigma_{1}\right)^{(4)}=\left(\sigma_{2}\right)^{(4)}$ and in this case $\left(v_{1}\right)^{(4)}=\left(\bar{v}_{1}\right)^{(4)}$ if in addition $\left(v_{0}\right)^{(4)}=$ $\left(v_{1}\right)^{(4)}$ then $v^{(4)}(t)=\left(v_{0}\right)^{(4)}$ and as a consequence $G_{24}(t)=\left(v_{0}\right)^{(4)} G_{25}(t)$ this also defines $\left(v_{0}\right)^{(4)}$ for the special case .

Analogously if $\left(b_{24}^{\prime \prime}\right)^{(4)}=\left(b_{25}^{\prime \prime}\right)^{(4)}$, then $\left(\tau_{1}\right)^{(4)}=\left(\tau_{2}\right)^{(4)}$ and then $\left(u_{1}\right)^{(4)}=\left(\bar{u}_{4}\right)^{(4)}$ if in addition $\left(u_{0}\right)^{(4)}=\left(u_{1}\right)^{(4)}$ then $T_{24}(t)=\left(u_{0}\right)^{(4)} T_{25}(t)$ This is an important consequence of the relation between $\left(v_{1}\right)^{(4)}$ and $\left(\bar{v}_{1}\right)^{(4)}$, and definition of $\left(u_{0}\right)^{(4)}$.

Proof : From concatenated set of equations we obtain

$\frac{d v^{(5)}}{d t}=\left(a_{28}\right)^{(5)}-\left(\left(a_{28}^{\prime}\right)^{(5)}-\left(a_{29}^{\prime}\right)^{(5)}+\left(a_{28}^{\prime \prime}\right)^{(5)}\left(T_{29}, t\right)\right)-\left(a_{29}^{\prime \prime}\right)^{(5)}\left(T_{29}, t\right) v^{(5)}-\left(a_{29}\right)^{(5)} v^{(5)}$

Definition of $v^{(5)}:-\quad v^{(5)}=\frac{G_{28}}{G_{29}}$

It follows

$-\left(\left(a_{29}\right)^{(5)}\left(v^{(5)}\right)^{2}+\left(\sigma_{2}\right)^{(5)} v^{(5)}-\left(a_{28}\right)^{(5)}\right) \leq \frac{d v^{(5)}}{d t} \leq-\left(\left(a_{29}\right)^{(5)}\left(v^{(5)}\right)^{2}+\left(\sigma_{1}\right)^{(5)} v^{(5)}-\left(a_{28}\right)^{(5)}\right)$

From which one obtains 
Definition of $\left(\bar{v}_{1}\right)^{(5)},\left(v_{0}\right)^{(5)}$ :-

(g) For $0<\left(v_{0}\right)^{(5)}=\frac{G_{28}^{0}}{G_{29}^{0}}<\left(v_{1}\right)^{(5)}<\left(\bar{v}_{1}\right)^{(5)}$

$$
v^{(5)}(t) \geq \frac{\left(v_{1}\right)^{(5)}+(C)^{(5)}\left(v_{2}\right)^{(5)} e^{\left[-\left(a_{29}\right)^{(5)}\left(\left(v_{1}\right)^{(5)}-\left(v_{0}\right)^{(5)}\right) t\right]}}{5+(C)^{(5)} e^{\left.-\left(a_{29}\right)^{(5)}\left(\left(v_{1}\right)^{(5)}-\left(v_{0}\right)^{(5)}\right) t\right]}} \quad, \quad(C)^{(5)}=\frac{\left(v_{1}\right)^{(5)}-\left(v_{0}\right)^{(5)}}{\left(v_{0}\right)^{(5)}-\left(v_{2}\right)^{(5)}}
$$

it follows $\left(v_{0}\right)^{(5)} \leq v^{(5)}(t) \leq\left(v_{1}\right)^{(5)}$

In the same manner, we get

$$
v^{(5)}(t) \leq \frac{\left(\bar{v}_{1}\right)^{(5)}+(\bar{C})^{(5)}\left(\bar{v}_{2}\right)^{(5)} e^{\left.\left[-\left(a_{29}\right)^{(5)}\left(\bar{v}_{1}\right)^{(5)}-\left(\bar{v}_{2}\right)^{(5)}\right) t\right]}}{5+(\bar{C})^{(5)} e^{\left.\left.-\left(a_{29}\right)^{(5)}\left(\bar{v}_{1}\right)^{(5)}-\left(\bar{v}_{2}\right)^{(5)}\right) t\right]}} \quad, \quad(\bar{C})^{(5)}=\frac{\left(\bar{v}_{1}\right)^{(5)}-\left(v_{0}\right)^{(5)}}{\left(v_{0}\right)^{(5)}-\left(\bar{v}_{2}\right)^{(5)}}
$$

From which we deduce $\left(v_{0}\right)^{(5)} \leq v^{(5)}(t) \leq\left(\bar{v}_{5}\right)^{(5)}$

(h) If $0<\left(v_{1}\right)^{(5)}<\left(v_{0}\right)^{(5)}=\frac{G_{28}^{0}}{G_{29}^{0}}<\left(\bar{v}_{1}\right)^{(5)}$ we find like in the previous case,

$$
\begin{gathered}
\left(v_{1}\right)^{(5)} \leq \frac{\left(v_{1}\right)^{(5)}+(C)^{(5)}\left(v_{2}\right)^{(5)} e^{\left.\left[-\left(a_{29}\right)^{(5)}\left(v_{1}\right)^{(5)}-\left(v_{2}\right)^{(5)}\right) t\right]}}{1+(C)^{(5)} e^{\left.\left.-\left(a_{29}\right)^{(5)}\left(v_{1}\right)^{(5)}-\left(v_{2}\right)^{(5)}\right) t\right]}} \leq v^{(5)}(t) \leq \\
\frac{\left(\bar{v}_{1}\right)^{(5)}+(\bar{C})^{(5)}\left(\bar{v}_{2}\right)^{(5)} e^{\left.\left[-\left(a_{29}\right)^{(5)}\left(\bar{v}_{1}\right)^{(5)}-\left(\bar{v}_{2}\right)^{(5)}\right) t\right]}}{1+(\bar{C})^{(5)} e^{\left.\left[-\left(a_{29}\right)^{(5)}\left(\bar{v}_{1}\right)^{(5)}-\left(\bar{v}_{2}\right)^{(5)}\right) t\right]}} \leq\left(\bar{v}_{1}\right)^{(5)}
\end{gathered}
$$

(i) If $0<\left(v_{1}\right)^{(5)} \leq\left(\bar{v}_{1}\right)^{(5)} \leq\left(v_{0}\right)^{(5)}=\frac{G_{28}^{0}}{G_{29}^{0}}$, we obtain

$$
\left(v_{1}\right)^{(5)} \leq v^{(5)}(t) \leq \frac{\left(\bar{v}_{1}\right)^{(5)}+\left(\bar{C}{ }^{(5)}\left(\bar{v}_{2}\right)^{(5)} e^{\left.\left[-\left(a_{29}\right)^{(5)}\left(\bar{v}_{1}\right)^{(5)}-\left(\bar{v}_{2}\right)^{(5)}\right) t\right]}\right.}{1+(\bar{C})^{(5)} e^{\left.\left[-\left(a_{29}\right)^{(5)}\left(\bar{v}_{1}\right)^{(5)}-\left(\bar{v}_{2}\right)^{(5)}\right) t\right]}} \leq\left(v_{0}\right)^{(5)}
$$

And so with the notation of the first part of condition (c), we have

Definition of $v^{(5)}(t)$ :-

$\left(m_{2}\right)^{(5)} \leq v^{(5)}(t) \leq\left(m_{1}\right)^{(5)}, v^{(5)}(t)=\frac{G_{28}(t)}{G_{29}(t)}$

In a completely analogous way, we obtain

Definition of $u^{(5)}(t)$ :-

$\left(\mu_{2}\right)^{(5)} \leq u^{(5)}(t) \leq\left(\mu_{1}\right)^{(5)}, \quad u^{(5)}(t)=\frac{T_{28}(t)}{T_{29}(t)}$

Now, using this result and replacing it in global equations we get easily the result stated in the theorem.

\section{$\underline{\text { Particular case : }}$}

If $\left(a_{28}^{\prime \prime}\right)^{(5)}=\left(a_{29}^{\prime \prime}\right)^{(5)}$, then $\left(\sigma_{1}\right)^{(5)}=\left(\sigma_{2}\right)^{(5)}$ and in this case $\left(v_{1}\right)^{(5)}=\left(\bar{v}_{1}\right)^{(5)}$ if in addition $\left(v_{0}\right)^{(5)}=$ $\left(v_{5}\right)^{(5)}$ then $v^{(5)}(t)=\left(v_{0}\right)^{(5)}$ and as a consequence $G_{28}(t)=\left(v_{0}\right)^{(5)} G_{29}(t)$ this also defines $\left(v_{0}\right)^{(5)}$ for the special case .

Analogously if $\left(b_{28}^{\prime \prime}\right)^{(5)}=\left(b_{29}^{\prime \prime}\right)^{(5)}$, then $\left(\tau_{1}\right)^{(5)}=\left(\tau_{2}\right)^{(5)}$ and then $\left(u_{1}\right)^{(5)}=\left(\bar{u}_{1}\right)^{(5)}$ if in addition $\left(u_{0}\right)^{(5)}=\left(u_{1}\right)^{(5)}$ then $T_{28}(t)=\left(u_{0}\right)^{(5)} T_{29}(t)$ This is an important consequence of the relation between $\left(v_{1}\right)^{(5)}$ and $\left(\bar{v}_{1}\right)^{(5)}$, and definition of $\left(u_{0}\right)^{(5)}$. 
Proof : From Global equations we obtain

$\frac{d v^{(6)}}{d t}=\left(a_{32}\right)^{(6)}-\left(\left(a_{32}^{\prime}\right)^{(6)}-\left(a_{33}^{\prime}\right)^{(6)}+\left(a_{32}^{\prime \prime}\right)^{(6)}\left(T_{33}, t\right)\right)-\left(a_{33}^{\prime \prime}\right)^{(6)}\left(T_{33}, t\right) v^{(6)}-\left(a_{33}\right)^{(6)} v^{(6)}$

Definition of $v^{(6)}:-\quad v^{(6)}=\frac{G_{32}}{G_{33}}$

It follows

$-\left(\left(a_{33}\right)^{(6)}\left(v^{(6)}\right)^{2}+\left(\sigma_{2}\right)^{(6)} v^{(6)}-\left(a_{32}\right)^{(6)}\right) \leq \frac{d v^{(6)}}{d t} \leq-\left(\left(a_{33}\right)^{(6)}\left(v^{(6)}\right)^{2}+\left(\sigma_{1}\right)^{(6)} v^{(6)}-\left(a_{32}\right)^{(6)}\right)$

From which one obtains

Definition of $\left(\bar{v}_{1}\right)^{(6)},\left(\nu_{0}\right)^{(6)}:-$

(j) For $0<\left(v_{0}\right)^{(6)}=\frac{G_{32}^{0}}{G_{33}^{0}}<\left(v_{1}\right)^{(6)}<\left(\bar{v}_{1}\right)^{(6)}$

$$
v^{(6)}(t) \geq \frac{\left(v_{1}\right)^{(6)}+(C)^{(6)}\left(v_{2}\right)^{(6)} e^{\left[-\left(a_{33}\right)^{(6)}\left(\left(v_{1}\right)^{(6)}-\left(v_{0}\right)^{(6)}\right) t\right]}}{1+(C)^{(6)} e^{\left[-\left(a_{33}\right)^{(6)}\left(\left(_{1}\right)^{(6)}-\left(v_{0}\right)^{(6)}\right) t\right]}} \quad, \quad(C)^{(6)}=\frac{\left(v_{1}\right)^{(6)}-\left(v_{0}\right)^{(6)}}{\left(v_{0}\right)^{(6)}-\left(v_{2}\right)^{(6)}}
$$

it follows $\left(v_{0}\right)^{(6)} \leq v^{(6)}(t) \leq\left(v_{1}\right)^{(6)}$

In the same manner, we get

$$
v^{(6)}(t) \leq \frac{\left(\bar{v}_{1}\right)^{(6)}+(\bar{C})^{(6)}\left(\bar{v}_{2}\right)^{(6)} e^{\left.\left[-\left(a_{33}\right)^{(6)}\left(\bar{v}_{1}\right)^{(6)}-\left(\bar{v}_{2}\right)^{(6)}\right) t\right]}}{1+(\bar{C})^{(6)} e^{\left.\left[-\left(a_{33}\right)^{(6)}\left(\bar{v}_{1}\right)^{(6)}-\left(\bar{v}_{2}\right)^{(6)}\right) t\right]}} \quad, \quad(\bar{C})^{(6)}=\frac{\left(\bar{v}_{1}\right)^{(6)}-\left(v_{0}\right)^{(6)}}{\left(v_{0}\right)^{(6)}-\left(\bar{v}_{2}\right)^{(6)}}
$$

From which we deduce $\left(v_{0}\right)^{(6)} \leq v^{(6)}(t) \leq\left(\bar{v}_{1}\right)^{(6)}$

(k) If $0<\left(v_{1}\right)^{(6)}<\left(v_{0}\right)^{(6)}=\frac{G_{32}^{0}}{G_{33}^{0}}<\left(\bar{v}_{1}\right)^{(6)}$ we find like in the previous case,

$$
\left(v_{1}\right)^{(6)} \leq \frac{\left(v_{1}\right)^{(6)}+(C)^{(6)}\left(v_{2}\right)^{(6)} e^{\left[-\left(a_{33}\right)^{(6)}\left(\left(v_{1}\right)^{(6)}-\left(v_{2}\right)^{(6)}\right) t\right]}}{1+(C)^{(6)} e^{\left[-\left(a_{33}\right)^{(6)}\left(\left(v_{1}\right)^{(6)}-\left(v_{2}\right)^{(6)}\right) t\right]}} \leq v^{(6)}(t) \leq
$$

$\frac{\left(\bar{v}_{1}\right)^{(6)}+(\bar{C})^{(6)}\left(\bar{v}_{2}\right)^{(6)} e^{\left[-\left(a_{33}\right)^{(6)}\left(\left(\bar{v}_{1}\right)^{(6)}-\left(\bar{v}_{2}\right)^{(6)}\right) t\right]}}{1+(\bar{C})^{(6)} e^{\left.\left[-\left(a_{33}\right)^{(6)}\left(\bar{v}_{1}\right)^{(6)}-\left(\bar{v}_{2}\right)^{(6)}\right) t\right]}} \leq\left(\bar{v}_{1}\right)^{(6)}$

(l) If $0<\left(v_{1}\right)^{(6)} \leq\left(\bar{v}_{1}\right)^{(6)} \leq\left(v_{0}\right)^{(6)}=\frac{G_{32}^{0}}{G_{33}^{0}}$, we obtain

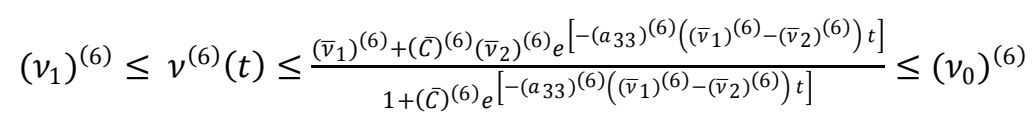

And so with the notation of the first part of condition (c), we have

Definition of $v^{(6)}(t)$ :-

$\left(m_{2}\right)^{(6)} \leq v^{(6)}(t) \leq\left(m_{1}\right)^{(6)}, v^{(6)}(t)=\frac{G_{32}(t)}{G_{33}(t)}$

In a completely analogous way, we obtain

Definition of $u^{(6)}(t)$ :- 
$\left(\mu_{2}\right)^{(6)} \leq u^{(6)}(t) \leq\left(\mu_{1}\right)^{(6)}, u^{(6)}(t)=\frac{T_{32}(t)}{T_{33}(t)}$

Now, using this result and replacing it in global equations we get easily the result stated in the theorem.

\section{Particular case :}

If $\left(a_{32}^{\prime \prime}\right)^{(6)}=\left(a_{33}^{\prime \prime}\right)^{(6)}$, then $\left(\sigma_{1}\right)^{(6)}=\left(\sigma_{2}\right)^{(6)}$ and in this case $\left(v_{1}\right)^{(6)}=\left(\bar{v}_{1}\right)^{(6)}$ if in addition $\left(v_{0}\right)^{(6)}=$ $\left(v_{1}\right)^{(6)}$ then $v^{(6)}(t)=\left(v_{0}\right)^{(6)}$ and as a consequence $G_{32}(t)=\left(v_{0}\right)^{(6)} G_{33}(t)$ this also defines $\left(v_{0}\right)^{(6)}$ for the special case

Analogously if $\left(b_{32}^{\prime \prime}\right)^{(6)}=\left(b_{33}^{\prime \prime}\right)^{(6)}$, then $\left(\tau_{1}\right)^{(6)}=\left(\tau_{2}\right)^{(6)}$ and then $\left(u_{1}\right)^{(6)}=\left(\bar{u}_{1}\right)^{(6)}$ if in addition $\left(u_{0}\right)^{(6)}=\left(u_{1}\right)^{(6)}$ then $T_{32}(t)=\left(u_{0}\right)^{(6)} T_{33}(t)$ This is an important consequence of the relation between $\left(v_{1}\right)^{(6)}$ and $\left(\bar{v}_{1}\right)^{(6)}$, and definition of $\left(u_{0}\right)^{(6)}$.

We can prove the following

Theorem 3: If $\left(a_{i}^{\prime \prime}\right)^{(1)}$ and $\left(b_{i}^{\prime \prime}\right)^{(1)}$ are independent on $t$, and the conditions

$\left(a_{13}^{\prime}\right)^{(1)}\left(a_{14}^{\prime}\right)^{(1)}-\left(a_{13}\right)^{(1)}\left(a_{14}\right)^{(1)}<0$

$\left(a_{13}^{\prime}\right)^{(1)}\left(a_{14}^{\prime}\right)^{(1)}-\left(a_{13}\right)^{(1)}\left(a_{14}\right)^{(1)}+\left(a_{13}\right)^{(1)}\left(p_{13}\right)^{(1)}+\left(a_{14}^{\prime}\right)^{(1)}\left(p_{14}\right)^{(1)}+\left(p_{13}\right)^{(1)}\left(p_{14}\right)^{(1)}>0$

$\left(b_{13}^{\prime}\right)^{(1)}\left(b_{14}^{\prime}\right)^{(1)}-\left(b_{13}\right)^{(1)}\left(b_{14}\right)^{(1)}>0$,

$\left(b_{13}^{\prime}\right)^{(1)}\left(b_{14}^{\prime}\right)^{(1)}-\left(b_{13}\right)^{(1)}\left(b_{14}\right)^{(1)}-\left(b_{13}^{\prime}\right)^{(1)}\left(r_{14}\right)^{(1)}-\left(b_{14}^{\prime}\right)^{(1)}\left(r_{14}\right)^{(1)}+\left(r_{13}\right)^{(1)}\left(r_{14}\right)^{(1)}<0$

with $\left(p_{13}\right)^{(1)},\left(r_{14}\right)^{(1)}$ as defined are satisfied, then the system

If $\left(a_{i}^{\prime \prime}\right)^{(2)}$ and $\left(b_{i}^{\prime \prime}\right)^{(2)}$ are independent on $\mathrm{t}$, and the conditions

$\left(a_{16}^{\prime}\right)^{(2)}\left(a_{17}^{\prime}\right)^{(2)}-\left(a_{16}\right)^{(2)}\left(a_{17}\right)^{(2)}<0$

$\left(a_{16}^{\prime}\right)^{(2)}\left(a_{17}^{\prime}\right)^{(2)}-\left(a_{16}\right)^{(2)}\left(a_{17}\right)^{(2)}+\left(a_{16}\right)^{(2)}\left(p_{16}\right)^{(2)}+\left(a_{17}^{\prime}\right)^{(2)}\left(p_{17}\right)^{(2)}+\left(p_{16}\right)^{(2)}\left(p_{17}\right)^{(2)}>0$

$\left(b_{16}^{\prime}\right)^{(2)}\left(b_{17}^{\prime}\right)^{(2)}-\left(b_{16}\right)^{(2)}\left(b_{17}\right)^{(2)}>0$,

$\left(b_{16}^{\prime}\right)^{(2)}\left(b_{17}^{\prime}\right)^{(2)}-\left(b_{16}\right)^{(2)}\left(b_{17}\right)^{(2)}-\left(b_{16}^{\prime}\right)^{(2)}\left(r_{17}\right)^{(2)}-\left(b_{17}^{\prime}\right)^{(2)}\left(r_{17}\right)^{(2)}+\left(r_{16}\right)^{(2)}\left(r_{17}\right)^{(2)}<0$

with $\left(p_{16}\right)^{(2)},\left(r_{17}\right)^{(2)}$ as defined are satisfied, then the system

: If $\left(a_{i}^{\prime \prime}\right)^{(3)}$ and $\left(b_{i}^{\prime \prime}\right)^{(3)}$ are independent on $t$, and the conditions

$\left(a_{20}^{\prime}\right)^{(3)}\left(a_{21}^{\prime}\right)^{(3)}-\left(a_{20}\right)^{(3)}\left(a_{21}\right)^{(3)}<0$

$\left(a_{20}^{\prime}\right)^{(3)}\left(a_{21}^{\prime}\right)^{(3)}-\left(a_{20}\right)^{(3)}\left(a_{21}\right)^{(3)}+\left(a_{20}\right)^{(3)}\left(p_{20}\right)^{(3)}+\left(a_{21}^{\prime}\right)^{(3)}\left(p_{21}\right)^{(3)}+\left(p_{20}\right)^{(3)}\left(p_{21}\right)^{(3)}>0$

$\left(b_{20}^{\prime}\right)^{(3)}\left(b_{21}^{\prime}\right)^{(3)}-\left(b_{20}\right)^{(3)}\left(b_{21}\right)^{(3)}>0$,

$\left(b_{20}^{\prime}\right)^{(3)}\left(b_{21}^{\prime}\right)^{(3)}-\left(b_{20}\right)^{(3)}\left(b_{21}\right)^{(3)}-\left(b_{20}^{\prime}\right)^{(3)}\left(r_{21}\right)^{(3)}-\left(b_{21}^{\prime}\right)^{(3)}\left(r_{21}\right)^{(3)}+\left(r_{20}\right)^{(3)}\left(r_{21}\right)^{(3)}<0$

with $\left(p_{20}\right)^{(3)},\left(r_{21}\right)^{(3)}$ as defined are satisfied, then the system

We can prove the following

If $\left(a_{i}^{\prime \prime}\right)^{(4)}$ and $\left(b_{i}^{\prime \prime}\right)^{(4)}$ are independent on $t$, and the conditions 
$\left(a_{24}^{\prime}\right)^{(4)}\left(a_{25}^{\prime}\right)^{(4)}-\left(a_{24}\right)^{(4)}\left(a_{25}\right)^{(4)}<0$

$\left(a_{24}^{\prime}\right)^{(4)}\left(a_{25}^{\prime}\right)^{(4)}-\left(a_{24}\right)^{(4)}\left(a_{25}\right)^{(4)}+\left(a_{24}\right)^{(4)}\left(p_{24}\right)^{(4)}+\left(a_{25}^{\prime}\right)^{(4)}\left(p_{25}\right)^{(4)}+\left(p_{24}\right)^{(4)}\left(p_{25}\right)^{(4)}>0$

$\left(b_{24}^{\prime}\right)^{(4)}\left(b_{25}^{\prime}\right)^{(4)}-\left(b_{24}\right)^{(4)}\left(b_{25}\right)^{(4)}>0$,

$\left(b_{24}^{\prime}\right)^{(4)}\left(b_{25}^{\prime}\right)^{(4)}-\left(b_{24}\right)^{(4)}\left(b_{25}\right)^{(4)}-\left(b_{24}^{\prime}\right)^{(4)}\left(r_{25}\right)^{(4)}-\left(b_{25}^{\prime}\right)^{(4)}\left(r_{25}\right)^{(4)}+\left(r_{24}\right)^{(4)}\left(r_{25}\right)^{(4)}<0$

with $\left(p_{24}\right)^{(4)},\left(r_{25}\right)^{(4)}$ as defined are satisfied, then the system

If $\left(a_{i}^{\prime \prime}\right)^{(5)}$ and $\left(b_{i}^{\prime \prime}\right)^{(5)}$ are independent on $t$, and the conditions

$\left(a_{28}^{\prime}\right)^{(5)}\left(a_{29}^{\prime}\right)^{(5)}-\left(a_{28}\right)^{(5)}\left(a_{29}\right)^{(5)}<0$

$\left(a_{28}^{\prime}\right)^{(5)}\left(a_{29}^{\prime}\right)^{(5)}-\left(a_{28}\right)^{(5)}\left(a_{29}\right)^{(5)}+\left(a_{28}\right)^{(5)}\left(p_{28}\right)^{(5)}+\left(a_{29}^{\prime}\right)^{(5)}\left(p_{29}\right)^{(5)}+\left(p_{28}\right)^{(5)}\left(p_{29}\right)^{(5)}>0$

$\left(b_{28}^{\prime}\right)^{(5)}\left(b_{29}^{\prime}\right)^{(5)}-\left(b_{28}\right)^{(5)}\left(b_{29}\right)^{(5)}>0$,

$\left(b_{28}^{\prime}\right)^{(5)}\left(b_{29}^{\prime}\right)^{(5)}-\left(b_{28}\right)^{(5)}\left(b_{29}\right)^{(5)}-\left(b_{28}^{\prime}\right)^{(5)}\left(r_{29}\right)^{(5)}-\left(b_{29}^{\prime}\right)^{(5)}\left(r_{29}\right)^{(5)}+\left(r_{28}\right)^{(5)}\left(r_{29}\right)^{(5)}<0$

with $\left(p_{28}\right)^{(5)},\left(r_{29}\right)^{(5)}$ as defined are satisfied, then the system

If $\left(a_{i}^{\prime \prime}\right)^{(6)}$ and $\left(b_{i}^{\prime \prime}\right)^{(6)}$ are independent on $t$, and the conditions

$\left(a_{32}^{\prime}\right)^{(6)}\left(a_{33}^{\prime}\right)^{(6)}-\left(a_{32}\right)^{(6)}\left(a_{33}\right)^{(6)}<0$

$\left(a_{32}^{\prime}\right)^{(6)}\left(a_{33}^{\prime}\right)^{(6)}-\left(a_{32}\right)^{(6)}\left(a_{33}\right)^{(6)}+\left(a_{32}\right)^{(6)}\left(p_{32}\right)^{(6)}+\left(a_{33}^{\prime}\right)^{(6)}\left(p_{33}\right)^{(6)}+\left(p_{32}\right)^{(6)}\left(p_{33}\right)^{(6)}>0$

$\left(b_{32}^{\prime}\right)^{(6)}\left(b_{33}^{\prime}\right)^{(6)}-\left(b_{32}\right)^{(6)}\left(b_{33}\right)^{(6)}>0$,

$\left(b_{32}^{\prime}\right)^{(6)}\left(b_{33}^{\prime}\right)^{(6)}-\left(b_{32}\right)^{(6)}\left(b_{33}\right)^{(6)}-\left(b_{32}^{\prime}\right)^{(6)}\left(r_{33}\right)^{(6)}-\left(b_{33}^{\prime}\right)^{(6)}\left(r_{33}\right)^{(6)}+\left(r_{32}\right)^{(6)}\left(r_{33}\right)^{(6)}<0$

with $\left(p_{32}\right)^{(6)},\left(r_{33}\right)^{(6)}$ as defined are satisfied, then the system Boolean satisfiability problem and $\mathrm{N}$ puzzle

$\left(a_{13}\right)^{(1)} G_{14}-\left[\left(a_{13}^{\prime}\right)^{(1)}+\left(a_{13}^{\prime \prime}\right)^{(1)}\left(T_{14}\right)\right] G_{13}=0$

$\left(a_{14}\right)^{(1)} G_{13}-\left[\left(a_{14}^{\prime}\right)^{(1)}+\left(a_{14}^{\prime \prime}\right)^{(1)}\left(T_{14}\right)\right] G_{14}=0$

$\left(a_{15}\right)^{(1)} G_{14}-\left[\left(a_{15}^{\prime}\right)^{(1)}+\left(a_{15}^{\prime \prime}\right)^{(1)}\left(T_{14}\right)\right] G_{15}=0$

$\left(b_{13}\right)^{(1)} T_{14}-\left[\left(b_{13}^{\prime}\right)^{(1)}-\left(b_{13}^{\prime \prime}\right)^{(1)}(G)\right] T_{13}=0$

$\left(b_{14}\right)^{(1)} T_{13}-\left[\left(b_{14}^{\prime}\right)^{(1)}-\left(b_{14}^{\prime \prime}\right)^{(1)}(G)\right] T_{14}=0$

$\left(b_{15}\right)^{(1)} T_{14}-\left[\left(b_{15}^{\prime}\right)^{(1)}-\left(b_{15}^{\prime \prime}\right)^{(1)}(G)\right] T_{15}=0$

has a unique positive solution, which is an equilibrium solution for the system

$\left(a_{16}\right)^{(2)} G_{17}-\left[\left(a_{16}^{\prime}\right)^{(2)}+\left(a_{16}^{\prime \prime}\right)^{(2)}\left(T_{17}\right)\right] G_{16}=0$

$\left(a_{17}\right)^{(2)} G_{16}-\left[\left(a_{17}^{\prime}\right)^{(2)}+\left(a_{17}^{\prime \prime}\right)^{(2)}\left(T_{17}\right)\right] G_{17}=0$

$\left(a_{18}\right)^{(2)} G_{17}-\left[\left(a_{18}^{\prime}\right)^{(2)}+\left(a_{18}^{\prime \prime}\right)^{(2)}\left(T_{17}\right)\right] G_{18}=0$ 
$\left(b_{16}\right)^{(2)} T_{17}-\left[\left(b_{16}^{\prime}\right)^{(2)}-\left(b_{16}^{\prime \prime}\right)^{(2)}\left(G_{19}\right)\right] T_{16}=0$

$\left(b_{17}\right)^{(2)} T_{16}-\left[\left(b_{17}^{\prime}\right)^{(2)}-\left(b_{17}^{\prime \prime}\right)^{(2)}\left(G_{19}\right)\right] T_{17}=0$

$\left(b_{18}\right)^{(2)} T_{17}-\left[\left(b_{18}^{\prime}\right)^{(2)}-\left(b_{18}^{\prime \prime}\right)^{(2)}\left(G_{19}\right)\right] T_{18}=0$

has a unique positive solution, which is an equilibrium solution for the system

$\left(a_{20}\right)^{(3)} G_{21}-\left[\left(a_{20}^{\prime}\right)^{(3)}+\left(a_{20}^{\prime \prime}\right)^{(3)}\left(T_{21}\right)\right] G_{20}=0$

$\left(a_{21}\right)^{(3)} G_{20}-\left[\left(a_{21}^{\prime}\right)^{(3)}+\left(a_{21}^{\prime \prime}\right)^{(3)}\left(T_{21}\right)\right] G_{21}=0$

$\left(a_{22}\right)^{(3)} G_{21}-\left[\left(a_{22}^{\prime}\right)^{(3)}+\left(a_{22}^{\prime \prime}\right)^{(3)}\left(T_{21}\right)\right] G_{22}=0$

$\left(b_{20}\right)^{(3)} T_{21}-\left[\left(b_{20}^{\prime}\right)^{(3)}-\left(b_{20}^{\prime \prime}\right)^{(3)}\left(G_{23}\right)\right] T_{20}=0$

$\left(b_{21}\right)^{(3)} T_{20}-\left[\left(b_{21}^{\prime}\right)^{(3)}-\left(b_{21}^{\prime \prime}\right)^{(3)}\left(G_{23}\right)\right] T_{21}=0$

$\left(b_{22}\right)^{(3)} T_{21}-\left[\left(b_{22}^{\prime}\right)^{(3)}-\left(b_{22}^{\prime \prime}\right)^{(3)}\left(G_{23}\right)\right] T_{22}=0$

has a unique positive solution, which is an equilibrium solution for the system

$$
\begin{array}{lr}
\left(a_{24}\right)^{(4)} G_{25}-\left[\left(a_{24}^{\prime}\right)^{(4)}+\left(a_{24}^{\prime \prime}\right)^{(4)}\left(T_{25}\right)\right] G_{24}=0 & 469 \\
\left(a_{25}\right)^{(4)} G_{24}-\left[\left(a_{25}^{\prime}\right)^{(4)}+\left(a_{25}^{\prime \prime}\right)^{(4)}\left(T_{25}\right)\right] G_{25}=0 & 470 \\
\left(a_{26}\right)^{(4)} G_{25}-\left[\left(a_{26}^{\prime}\right)^{(4)}+\left(a_{26}^{\prime \prime}\right)^{(4)}\left(T_{25}\right)\right] G_{26}=0 & 471 \\
\left(b_{24}\right)^{(4)} T_{25}-\left[\left(b_{24}^{\prime}\right)^{(4)}-\left(b_{24}^{\prime \prime}\right)^{(4)}\left(\left(G_{27}\right)\right)\right] T_{24}=0 & 472 \\
\left(b_{25}\right)^{(4)} T_{24}-\left[\left(b_{25}^{\prime}\right)^{(4)}-\left(b_{25}^{\prime \prime}\right)^{(4)}\left(\left(G_{27}\right)\right)\right] T_{25}=0 & 473 \\
\left(b_{26}\right)^{(4)} T_{25}-\left[\left(b_{26}^{\prime}\right)^{(4)}-\left(b_{26}^{\prime \prime}\right)^{(4)}\left(\left(G_{27}\right)\right)\right] T_{26}=0 & 474
\end{array}
$$

has a unique positive solution, which is an equilibrium solution for the system

$$
\left(a_{28}\right)^{(5)} G_{29}-\left[\left(a_{28}^{\prime}\right)^{(5)}+\left(a_{28}^{\prime \prime}\right)^{(5)}\left(T_{29}\right)\right] G_{28}=0
$$

$\left(a_{29}\right)^{(5)} G_{28}-\left[\left(a_{29}^{\prime}\right)^{(5)}+\left(a_{29}^{\prime \prime}\right)^{(5)}\left(T_{29}\right)\right] G_{29}=0$

$\left(a_{30}\right)^{(5)} G_{29}-\left[\left(a_{30}^{\prime}\right)^{(5)}+\left(a_{30}^{\prime \prime}\right)^{(5)}\left(T_{29}\right)\right] G_{30}=0$

$\left(b_{28}\right)^{(5)} T_{29}-\left[\left(b_{28}^{\prime}\right)^{(5)}-\left(b_{28}^{\prime \prime}\right)^{(5)}\left(G_{31}\right)\right] T_{28}=0$

$\left(b_{29}\right)^{(5)} T_{28}-\left[\left(b_{29}^{\prime}\right)^{(5)}-\left(b_{29}^{\prime \prime}\right)^{(5)}\left(G_{31}\right)\right] T_{29}=0$

$\left(b_{30}\right)^{(5)} T_{29}-\left[\left(b_{30}^{\prime}\right)^{(5)}-\left(b_{30}^{\prime \prime}\right)^{(5)}\left(G_{31}\right)\right] T_{30}=0$

has a unique positive solution, which is an equilibrium solution for the system 
$\left(a_{32}\right)^{(6)} G_{33}-\left[\left(a_{32}^{\prime}\right)^{(6)}+\left(a_{32}^{\prime \prime}\right)^{(6)}\left(T_{33}\right)\right] G_{32}=0$

$\left(a_{33}\right)^{(6)} G_{32}-\left[\left(a_{33}^{\prime}\right)^{(6)}+\left(a_{33}^{\prime \prime}\right)^{(6)}\left(T_{33}\right)\right] G_{33}=0$

$\left(a_{34}\right)^{(6)} G_{33}-\left[\left(a_{34}^{\prime}\right)^{(6)}+\left(a_{34}^{\prime \prime}\right)^{(6)}\left(T_{33}\right)\right] G_{34}=0$

$\left(b_{32}\right)^{(6)} T_{33}-\left[\left(b_{32}^{\prime}\right)^{(6)}-\left(b_{32}^{\prime \prime}\right)^{(6)}\left(G_{35}\right)\right] T_{32}=0$

$\left(b_{33}\right)^{(6)} T_{32}-\left[\left(b_{33}^{\prime}\right)^{(6)}-\left(b_{33}^{\prime \prime}\right)^{(6)}\left(G_{35}\right)\right] T_{33}=0$

$\left(b_{34}\right)^{(6)} T_{33}-\left[\left(b_{34}^{\prime}\right)^{(6)}-\left(b_{34}^{\prime \prime}\right)^{(6)}\left(G_{35}\right)\right] T_{34}=0$

has a unique positive solution, which is an equilibrium solution for the system

\section{Proof:}

(a) Indeed the first two equations have a nontrivial solution $G_{13}, G_{14}$ if

$F(T)=$

$\left(a_{13}^{\prime}\right)^{(1)}\left(a_{14}^{\prime}\right)^{(1)}-\left(a_{13}\right)^{(1)}\left(a_{14}\right)^{(1)}+\left(a_{13}^{\prime}\right)^{(1)}\left(a_{14}^{\prime \prime}\right)^{(1)}\left(T_{14}\right)+\left(a_{14}^{\prime}\right)^{(1)}\left(a_{13}^{\prime \prime}\right)^{(1)}\left(T_{14}\right)+$ $\left(a_{13}^{\prime \prime}\right)^{(1)}\left(T_{14}\right)\left(a_{14}^{\prime \prime}\right)^{(1)}\left(T_{14}\right)=0$

Indeed the first two equations have a nontrivial solution $G_{16}, G_{17}$ if

$\mathrm{F}\left(T_{19}\right)=$

$\left(a_{16}^{\prime}\right)^{(2)}\left(a_{17}^{\prime}\right)^{(2)}-\left(a_{16}\right)^{(2)}\left(a_{17}\right)^{(2)}+\left(a_{16}^{\prime}\right)^{(2)}\left(a_{17}^{\prime \prime}\right)^{(2)}\left(T_{17}\right)+\left(a_{17}^{\prime}\right)^{(2)}\left(a_{16}^{\prime \prime}\right)^{(2)}\left(T_{17}\right)+$ $\left(a_{16}^{\prime \prime}\right)^{(2)}\left(T_{17}\right)\left(a_{17}^{\prime \prime}\right)^{(2)}\left(T_{17}\right)=0$

(a) Indeed the first two equations have a nontrivial solution $G_{20}, G_{21}$ if

$F\left(T_{23}\right)=$

$\left(a_{20}^{\prime}\right)^{(3)}\left(a_{21}^{\prime}\right)^{(3)}-\left(a_{20}\right)^{(3)}\left(a_{21}\right)^{(3)}+\left(a_{20}^{\prime}\right)^{(3)}\left(a_{21}^{\prime \prime}\right)^{(3)}\left(T_{21}\right)+\left(a_{21}^{\prime}\right)^{(3)}\left(a_{20}^{\prime \prime}\right)^{(3)}\left(T_{21}\right)+$ $\left(a_{20}^{\prime \prime}\right)^{(3)}\left(T_{21}\right)\left(a_{21}^{\prime \prime}\right)^{(3)}\left(T_{21}\right)=0$

(a) Indeed the first two equations have a nontrivial solution $G_{24}, G_{25}$ if

$F\left(T_{27}\right)=$

$\left(a_{24}^{\prime}\right)^{(4)}\left(a_{25}^{\prime}\right)^{(4)}-\left(a_{24}\right)^{(4)}\left(a_{25}\right)^{(4)}+\left(a_{24}^{\prime}\right)^{(4)}\left(a_{25}^{\prime \prime}\right)^{(4)}\left(T_{25}\right)+\left(a_{25}^{\prime}\right)^{(4)}\left(a_{24}^{\prime \prime}\right)^{(4)}\left(T_{25}\right)+$ $\left(a_{24}^{\prime \prime}\right)^{(4)}\left(T_{25}\right)\left(a_{25}^{\prime \prime}\right)^{(4)}\left(T_{25}\right)=0$

(a) Indeed the first two equations have a nontrivial solution $G_{28}, G_{29}$ if

$$
\begin{aligned}
& F\left(T_{31}\right)= \\
& \left(a_{28}^{\prime}\right)^{(5)}\left(a_{29}^{\prime}\right)^{(5)}-\left(a_{28}\right)^{(5)}\left(a_{29}\right)^{(5)}+\left(a_{28}^{\prime}\right)^{(5)}\left(a_{29}^{\prime \prime}\right)^{(5)}\left(T_{29}\right)+\left(a_{29}^{\prime}\right)^{(5)}\left(a_{28}^{\prime \prime}\right)^{(5)}\left(T_{29}\right)+ \\
& \left(a_{28}^{\prime \prime}\right)^{(5)}\left(T_{29}\right)\left(a_{29}^{\prime \prime}\right)^{(5)}\left(T_{29}\right)=0
\end{aligned}
$$

(a) Indeed the first two equations have a nontrivial solution $G_{32}, G_{33}$ if

$$
\begin{aligned}
& F\left(T_{35}\right)= \\
& \left(a_{32}^{\prime}\right)^{(6)}\left(a_{33}^{\prime}\right)^{(6)}-\left(a_{32}\right)^{(6)}\left(a_{33}\right)^{(6)}+\left(a_{32}^{\prime}\right)^{(6)}\left(a_{33}^{\prime \prime}\right)^{(6)}\left(T_{33}\right)+\left(a_{33}^{\prime}\right)^{(6)}\left(a_{32}^{\prime \prime}\right)^{(6)}\left(T_{33}\right)+
\end{aligned}
$$


$\left(a_{32}^{\prime \prime}\right)^{(6)}\left(T_{33}\right)\left(a_{33}^{\prime \prime}\right)^{(6)}\left(T_{33}\right)=0$

Definition and uniqueness of $\mathrm{T}_{14}^{*}$ :-

After hypothesis $f(0)<0, f(\infty)>0$ and the functions $\left(a_{i}^{\prime \prime}\right)^{(1)}\left(T_{14}\right)$ being increasing, it follows that there exists a unique $T_{14}^{*}$ for which $f\left(T_{14}^{*}\right)=0$. With this value, we obtain from the three first equations

$G_{13}=\frac{\left(a_{13}\right)^{(1)} G_{14}}{\left[\left(a_{13}^{\prime}\right)^{(1)}+\left(a_{13}^{\prime \prime}\right)^{(1)}\left(T_{14}^{*}\right)\right]} \quad, \quad G_{15}=\frac{\left(a_{15}\right)^{(1)} G_{14}}{\left[\left(a_{15}^{\prime}\right)^{(1)}+\left(a_{15}^{\prime \prime}\right)^{(1)}\left(T_{14}^{*}\right)\right]}$

Definition and uniqueness of $\mathrm{T}_{17}^{*}$ :-

After hypothesis $f(0)<0, f(\infty)>0$ and the functions $\left(a_{i}^{\prime \prime}\right)^{(2)}\left(T_{17}\right)$ being increasing, it follows that there exists a unique $\mathrm{T}_{17}^{*}$ for which $f\left(\mathrm{~T}_{17}^{*}\right)=0$. With this value, we obtain from the three first equations

$G_{16}=\frac{\left(a_{16}\right)^{(2)} \mathrm{G}_{17}}{\left[\left(a_{16}^{\prime}\right)^{(2)}+\left(a_{16}^{\prime \prime}\right)^{(2)}\left(\mathrm{T}_{17}^{*}\right)\right]} \quad, \quad G_{18}=\frac{\left(a_{18}\right)^{(2)} \mathrm{G}_{17}}{\left[\left(a_{18}^{\prime}\right)^{(2)}+\left(a_{18}^{\prime \prime}\right)^{(2)}\left(\mathrm{T}_{17}^{*}\right)\right]}$

Definition and uniqueness of $\mathrm{T}_{21}^{*}$ :-

After hypothesis $f(0)<0, f(\infty)>0$ and the functions $\left(a_{i}^{\prime \prime}\right)^{(1)}\left(T_{21}\right)$ being increasing, it follows that there exists a unique $T_{21}^{*}$ for which $f\left(T_{21}^{*}\right)=0$. With this value, we obtain from the three first equations

$G_{20}=\frac{\left(a_{20}\right)^{(3)} G_{21}}{\left[\left(a_{20}^{\prime}\right)^{(3)}+\left(a_{20}^{\prime \prime}\right)^{(3)}\left(T_{21}^{*}\right)\right]} \quad, \quad G_{22}=\frac{\left(a_{22}\right)^{(3)} G_{21}}{\left[\left(a_{22}^{\prime}\right)^{(3)}+\left(a_{22}^{\prime \prime}\right)^{(3)}\left(T_{21}^{*}\right)\right]}$

Definition and uniqueness of $\mathrm{T}_{25}^{*}:-$

After hypothesis $f(0)<0, f(\infty)>0$ and the functions $\left(a_{i}^{\prime \prime}\right)^{(4)}\left(T_{25}\right)$ being increasing, it follows that there exists a unique $T_{25}^{*}$ for which $f\left(T_{25}^{*}\right)=0$. With this value, we obtain from the three first equations

$G_{24}=\frac{\left(a_{24}\right)^{(4)} G_{25}}{\left[\left(a_{24}^{\prime}\right)^{(4)}+\left(a_{24}^{\prime \prime}\right)^{(4)}\left(T_{25}^{*}\right)\right]} \quad, \quad G_{26}=\frac{\left(a_{26}\right)^{(4)} G_{25}}{\left[\left(a_{26}^{\prime}\right)^{(4)}+\left(a_{26}^{\prime \prime}\right)^{(4)}\left(T_{25}^{*}\right)\right]}$

Definition and uniqueness of $\mathrm{T}_{29}^{*}$ :-

After hypothesis $f(0)<0, f(\infty)>0$ and the functions $\left(a_{i}^{\prime \prime}\right)^{(5)}\left(T_{29}\right)$ being increasing, it follows that there exists a unique $T_{29}^{*}$ for which $f\left(T_{29}^{*}\right)=0$. With this value, we obtain from the three first equations

$G_{28}=\frac{\left(a_{28}\right)^{(5)} G_{29}}{\left[\left(a_{28}^{\prime}\right)^{(5)}+\left(a_{28}^{\prime \prime}\right)^{(5)}\left(T_{29}^{*}\right)\right]} \quad, \quad G_{30}=\frac{\left(a_{30}\right)^{(5)} G_{29}}{\left[\left(a_{30}^{\prime}\right)^{(5)}+\left(a_{30}^{\prime \prime}\right)^{(5)}\left(T_{29}^{*}\right)\right]}$

Definition and uniqueness of $\mathrm{T}_{33}^{*}:-$

After hypothesis $f(0)<0, f(\infty)>0$ and the functions $\left(a_{i}^{\prime \prime}\right)^{(6)}\left(T_{33}\right)$ being increasing, it follows that there exists a unique $T_{33}^{*}$ for which $f\left(T_{33}^{*}\right)=0$. With this value, we obtain from the three first equations

$G_{32}=\frac{\left(a_{32}\right)^{(6)} G_{33}}{\left[\left(a_{32}^{\prime}\right)^{(6)}+\left(a_{32}^{\prime \prime}\right)^{(6)}\left(T_{33}^{*}\right)\right]} \quad, \quad G_{34}=\frac{\left(a_{34}\right)^{(6)} G_{33}}{\left[\left(a_{34}^{\prime}\right)^{(6)}+\left(a_{34}^{\prime \prime}\right)^{(6)}\left(T_{33}^{*}\right)\right]}$

(e) By the same argument, the equations of global system admit solutions $G_{13}, G_{14}$ if

$$
\begin{aligned}
& \varphi(G)=\left(b_{13}^{\prime}\right)^{(1)}\left(b_{14}^{\prime}\right)^{(1)}-\left(b_{13}\right)^{(1)}\left(b_{14}\right)^{(1)}- \\
& {\left[\left(b_{13}^{\prime}\right)^{(1)}\left(b_{14}^{\prime \prime}\right)^{(1)}(G)+\left(b_{14}^{\prime}\right)^{(1)}\left(b_{13}^{\prime \prime}\right)^{(1)}(G)\right]+\left(b_{13}^{\prime \prime}\right)^{(1)}(G)\left(b_{14}^{\prime \prime}\right)^{(1)}(G)=0}
\end{aligned}
$$


Where in $G\left(G_{13}, G_{14}, G_{15}\right), G_{13}, G_{15}$ must be replaced by their values from 96 . It is easy to see that $\varphi$ is a decreasing function in $G_{14}$ taking into account the hypothesis $\varphi(0)>0, \varphi(\infty)<0$ it follows that there exists a unique $G_{14}^{*}$ such that $\varphi\left(G^{*}\right)=0$

(f) By the same argument, the equations 92,93 admit solutions $G_{16}, G_{17}$ if

$$
\begin{aligned}
& \varphi\left(G_{19}\right)=\left(b_{16}^{\prime}\right)^{(2)}\left(b_{17}^{\prime}\right)^{(2)}-\left(b_{16}\right)^{(2)}\left(b_{17}\right)^{(2)}- \\
& {\left[\left(b_{16}^{\prime}\right)^{(2)}\left(b_{17}^{\prime \prime}\right)^{(2)}\left(G_{19}\right)+\left(b_{17}^{\prime}\right)^{(2)}\left(b_{16}^{\prime \prime}\right)^{(2)}\left(G_{19}\right)\right]+\left(b_{16}^{\prime \prime}\right)^{(2)}\left(G_{19}\right)\left(b_{17}^{\prime \prime}\right)^{(2)}\left(G_{19}\right)=0}
\end{aligned}
$$

Where in $\left(G_{19}\right)\left(G_{16}, G_{17}, G_{18}\right), G_{16}, G_{18}$ must be replaced by their values from 96 . It is easy to see that $\varphi$ is a decreasing function in $G_{17}$ taking into account the hypothesis $\varphi(0)>0, \varphi(\infty)<0$ it follows that there exists a unique $\mathrm{G}_{14}^{*}$ such that $\varphi\left(\left(G_{19}\right)^{*}\right)=0$

(g) By the same argument, the equations of the global system admit solutions $G_{20}, G_{21}$ if

$$
\begin{aligned}
& \varphi\left(G_{23}\right)=\left(b_{20}^{\prime}\right)^{(3)}\left(b_{21}^{\prime}\right)^{(3)}-\left(b_{20}\right)^{(3)}\left(b_{21}\right)^{(3)}- \\
& {\left[\left(b_{20}^{\prime}\right)^{(3)}\left(b_{21}^{\prime \prime}\right)^{(3)}\left(G_{23}\right)+\left(b_{21}^{\prime}\right)^{(3)}\left(b_{20}^{\prime \prime}\right)^{(3)}\left(G_{23}\right)\right]+\left(b_{20}^{\prime \prime}\right)^{(3)}\left(G_{23}\right)\left(b_{21}^{\prime \prime}\right)^{(3)}\left(G_{23}\right)=0}
\end{aligned}
$$

Where in $G_{23}\left(G_{20}, G_{21}, G_{22}\right), G_{20}, G_{22}$ must be replaced by their values from 96 . It is easy to see that $\varphi$ is a decreasing function in $G_{21}$ taking into account the hypothesis $\varphi(0)>0, \varphi(\infty)<0$ it follows that there exists a unique $G_{21}^{*}$ such that $\varphi\left(\left(G_{23}\right)^{*}\right)=0$

(h) By the same argument, the equations of the global system admit solutions $G_{24}, G_{25}$ if

$$
\begin{aligned}
& \varphi\left(G_{27}\right)=\left(b_{24}^{\prime}\right)^{(4)}\left(b_{25}^{\prime}\right)^{(4)}-\left(b_{24}\right)^{(4)}\left(b_{25}\right)^{(4)}- \\
& {\left[\left(b_{24}^{\prime}\right)^{(4)}\left(b_{25}^{\prime \prime}\right)^{(4)}\left(G_{27}\right)+\left(b_{25}^{\prime}\right)^{(4)}\left(b_{24}^{\prime \prime}\right)^{(4)}\left(G_{27}\right)\right]+\left(b_{24}^{\prime \prime}\right)^{(4)}\left(G_{27}\right)\left(b_{25}^{\prime \prime}\right)^{(4)}\left(G_{27}\right)=0}
\end{aligned}
$$

Where in $\left(G_{27}\right)\left(G_{24}, G_{25}, G_{26}\right), G_{24}, G_{26}$ must be replaced by their values . It is easy to see that $\varphi$ is a decreasing function in $G_{25}$ taking into account the hypothesis $\varphi(0)>0, \varphi(\infty)<0$ it follows that there exists a unique $G_{25}^{*}$ such that $\varphi\left(\left(G_{27}\right)^{*}\right)=0$

(i) By the same argument, the global equations admit solutions $G_{28}, G_{29}$ if

$\varphi\left(G_{31}\right)=\left(b_{28}^{\prime}\right)^{(5)}\left(b_{29}^{\prime}\right)^{(5)}-\left(b_{28}\right)^{(5)}\left(b_{29}\right)^{(5)}-$

$\left[\left(b_{28}^{\prime}\right)^{(5)}\left(b_{29}^{\prime \prime}\right)^{(5)}\left(G_{31}\right)+\left(b_{29}^{\prime}\right)^{(5)}\left(b_{28}^{\prime \prime}\right)^{(5)}\left(G_{31}\right)\right]+\left(b_{28}^{\prime \prime}\right)^{(5)}\left(G_{31}\right)\left(b_{29}^{\prime \prime}\right)^{(5)}\left(G_{31}\right)=0$

Where in $\left(G_{31}\right)\left(G_{28}, G_{29}, G_{30}\right), G_{28}, G_{30}$ must be replaced by their values from 96 . It is easy to see that $\varphi$ is a decreasing function in $G_{29}$ taking into account the hypothesis $\varphi(0)>0, \varphi(\infty)<0$ it follows that there exists a unique $G_{29}^{*}$ such that $\varphi\left(\left(G_{31}\right)^{*}\right)=0$

(j) By the same argument, the global equations admit solutions $G_{32}, G_{33}$ if $\varphi\left(G_{35}\right)=\left(b_{32}^{\prime}\right)^{(6)}\left(b_{33}^{\prime}\right)^{(6)}-\left(b_{32}\right)^{(6)}\left(b_{33}\right)^{(6)}-$

$\left[\left(b_{32}^{\prime}\right)^{(6)}\left(b_{33}^{\prime \prime}\right)^{(6)}\left(G_{35}\right)+\left(b_{33}^{\prime}\right)^{(6)}\left(b_{32}^{\prime \prime}\right)^{(6)}\left(G_{35}\right)\right]+\left(b_{32}^{\prime \prime}\right)^{(6)}\left(G_{35}\right)\left(b_{33}^{\prime \prime}\right)^{(6)}\left(G_{35}\right)=0$

Where in $\left(G_{35}\right)\left(G_{32}, G_{33}, G_{34}\right), G_{32}, G_{34}$ must be replaced by their values from 96 . It is easy to see that $\varphi$ is a decreasing function in $G_{33}$ taking into account the hypothesis $\varphi(0)>0, \varphi(\infty)<0$ it follows that there exists a 
unique $G_{33}^{*}$ such that $\varphi\left(G^{*}\right)=0$

Finally we obtain the unique solution of the global system:

$G_{14}^{*}$ given by $\varphi\left(G^{*}\right)=0, T_{14}^{*}$ given by $f\left(T_{14}^{*}\right)=0$ and

$G_{13}^{*}=\frac{\left(a_{13}\right)^{(1)} G_{14}^{*}}{\left[\left(a_{13}^{\prime}\right)^{(1)}+\left(a_{13}^{\prime \prime}\right)^{(1)}\left(T_{14}^{*}\right)\right]} \quad, \quad G_{15}^{*}=\frac{\left(a_{15}\right)^{(1)} G_{14}^{*}}{\left[\left(a_{15}^{\prime}\right)^{(1)}+\left(a_{15}^{\prime \prime}\right)^{(1)}\left(T_{14}^{*}\right)\right]}$

$T_{13}^{*}=\frac{\left(b_{13}\right)^{(1)} T_{14}^{*}}{\left[\left(b_{13}^{\prime}\right)^{(1)}-\left(b_{13}^{\prime \prime}\right)^{(1)}\left(G^{*}\right)\right]} \quad, \quad T_{15}^{*}=\frac{\left(b_{15}\right)^{(1)} T_{14}^{*}}{\left[\left(b_{15}^{\prime}\right)^{(1)}-\left(b_{15}^{\prime \prime}\right)^{(1)}\left(G^{*}\right)\right]}$

Obviously, these values represent an equilibrium solution

Finally we obtain the unique solution

$\mathrm{G}_{17}^{*}$ given by $\varphi\left(\left(G_{19}\right)^{*}\right)=0, \mathrm{~T}_{17}^{*}$ given by $f\left(\mathrm{~T}_{17}^{*}\right)=0$ and

$\mathrm{G}_{16}^{*}=\frac{\left(\mathrm{a}_{16}\right)^{(2)} \mathrm{G}_{17}^{*}}{\left[\left(\mathrm{a}_{16}^{\prime}\right)^{(2)}+\left(\mathrm{a}_{16}^{\prime \prime}\right)^{(2)}\left(\mathrm{T}_{17}^{*}\right)\right]} \quad, \quad \mathrm{G}_{18}^{*}=\frac{\left(\mathrm{a}_{18}\right)^{(2)} \mathrm{G}_{17}^{*}}{\left[\left(\mathrm{a}_{18}^{\prime}\right)^{(2)}+\left(\mathrm{a}_{18}^{\prime \prime}\right)^{(2)}\left(\mathrm{T}_{17}^{*}\right)\right]}$

$\mathrm{T}_{16}^{*}=\frac{\left(\mathrm{b}_{16}\right)^{(2)} \mathrm{T}_{17}^{*}}{\left[\left(\mathrm{~b}_{16}^{\prime}\right)^{(2)}-\left(\mathrm{b}_{16}^{\prime \prime}\right)^{(2)}\left(\left(G_{19}\right)^{*}\right)\right]} \quad, \quad \mathrm{T}_{18}^{*}=\frac{\left(\mathrm{b}_{18}\right)^{(2)} \mathrm{T}_{17}^{*}}{\left[\left(\mathrm{~b}_{18}^{\prime}\right)^{(2)}-\left(\mathrm{b}_{18}^{\prime \prime}\right)^{(2)}\left(\left(G_{19}\right)^{*}\right)\right]}$

Obviously, these values represent an equilibrium solution

Finally we obtain the unique solution

$G_{21}^{*}$ given by $\varphi\left(\left(G_{23}\right)^{*}\right)=0, T_{21}^{*}$ given by $f\left(T_{21}^{*}\right)=0$ and

$$
\begin{array}{ll}
G_{20}^{*}=\frac{\left(a_{20}\right)^{(3)} G_{21}^{*}}{\left[\left(a_{20}^{\prime}\right)^{(3)}+\left(a_{20}^{\prime \prime}\right)^{(3)}\left(T_{21}^{*}\right)\right]} \quad, \quad G_{22}^{*}=\frac{\left(a_{22}\right)^{(3)} G_{21}^{*}}{\left[\left(a_{22}^{\prime}\right)^{(3)}+\left(a_{22}^{\prime \prime}\right)^{(3)}\left(T_{21}^{*}\right)\right]} \\
T_{20}^{*}=\frac{\left(b_{20}\right)^{(3)} T_{21}^{*}}{\left[\left(b_{20}^{\prime}\right)^{(3)}-\left(b_{20}^{\prime \prime}\right)^{(3)}\left(G_{23}{ }^{*}\right)\right]} \quad, \quad T_{22}^{*}=\frac{\left(b_{22}\right)^{(3)} T_{21}^{*}}{\left[\left(b_{22}^{\prime}\right)^{(3)}-\left(b_{22}^{\prime \prime}\right)^{(3)}\left(G_{23}{ }^{*}\right)\right]}
\end{array}
$$

Obviously, these values represent an equilibrium solution

Finally we obtain the unique solution

$G_{25}^{*}$ given by $\varphi\left(G_{27}\right)=0, T_{25}^{*}$ given by $f\left(T_{25}^{*}\right)=0$ and

$$
\begin{aligned}
& G_{24}^{*}=\frac{\left(a_{24}\right)^{(4)} G_{25}^{*}}{\left[\left(a_{24}^{\prime}\right)^{(4)}+\left(a_{24}^{\prime \prime}\right)^{(4)}\left(T_{25}^{*}\right)\right]} \quad, \quad G_{26}^{*}=\frac{\left(a_{26}\right)^{(4)} G_{25}^{*}}{\left[\left(a_{26}^{\prime}\right)^{(4)}+\left(a_{26}^{\prime \prime}\right)^{(4)}\left(T_{25}^{*}\right)\right]} \\
& T_{24}^{*}=\frac{\left(b_{24}\right)^{(4)} T_{25}^{*}}{\left[\left(b_{24}^{\prime}\right)^{(4)}-\left(b_{24}^{\prime \prime}\right)^{(4)}\left(\left(G_{27}\right)^{*}\right)\right]} \quad, \quad T_{26}^{*}=\frac{\left(b_{26}\right)^{(4)} T_{25}^{*}}{\left[\left(b_{26}^{\prime}\right)^{(4)}-\left(b_{26}^{\prime \prime}\right)^{(4)}\left(\left(G_{27}\right)^{*}\right)\right]}
\end{aligned}
$$

Obviously, these values represent an equilibrium solution

Finally we obtain the unique solution

$G_{29}^{*}$ given by $\varphi\left(\left(G_{31}\right)^{*}\right)=0, T_{29}^{*}$ given by $f\left(T_{29}^{*}\right)=0$ and

$$
G_{28}^{*}=\frac{\left(a_{28}\right)^{(5)} G_{29}^{*}}{\left[\left(a_{28}^{\prime}\right)^{(5)}+\left(a_{28}^{\prime \prime}\right)^{(5)}\left(T_{29}^{*}\right)\right]} \quad, \quad G_{30}^{*}=\frac{\left(a_{30}\right)^{(5)} G_{29}^{*}}{\left[\left(a_{30}^{\prime}\right)^{(5)}+\left(a_{30}^{\prime \prime}\right)^{(5)}\left(T_{29}^{*}\right)\right]}
$$


$T_{28}^{*}=\frac{\left(b_{28}\right)^{(5)} T_{29}^{*}}{\left[\left(b_{28}^{\prime}\right)^{(5)}-\left(b_{28}^{\prime \prime}\right)^{(5)}\left(\left(G_{31}\right)^{*}\right)\right]} \quad, \quad T_{30}^{*}=\frac{\left(b_{30}\right)^{(5)} T_{29}^{*}}{\left[\left(b_{30}^{\prime}\right)^{(5)}-\left(b_{30}^{\prime \prime}\right)^{(5)}\left(\left(G_{31}\right)^{*}\right)\right]}$

Obviously, these values represent an equilibrium solution

Finally we obtain the unique solution

$G_{33}^{*}$ given by $\varphi\left(\left(G_{35}\right)^{*}\right)=0, T_{33}^{*}$ given by $f\left(T_{33}^{*}\right)=0$ and

$$
\begin{aligned}
& G_{32}^{*}=\frac{\left(a_{32}\right)^{(6)} G_{33}^{*}}{\left[\left(a_{32}^{\prime}\right)^{(6)}+\left(a_{32}^{\prime \prime}\right)^{(6)}\left(T_{33}^{*}\right)\right]} \quad, \quad G_{34}^{*}=\frac{\left(a_{34}\right)^{(6)} G_{33}^{*}}{\left[\left(a_{34}^{\prime}\right)^{(6)}+\left(a_{34}^{\prime}\right)^{(6)}\left(T_{33}^{*}\right)\right]} \\
& T_{32}^{*}=\frac{\left(b_{32}\right)^{(6)} T_{33}^{*}}{\left[\left(b_{32}^{\prime}\right)^{(6)}-\left(b_{32}^{\prime \prime}\right)^{(6)}\left(\left(G_{35}\right)^{*}\right)\right]} \quad, \quad T_{34}^{*}=\frac{\left(b_{34}\right)^{(6)} T_{33}^{*}}{\left[\left(b_{34}^{\prime}\right)^{(6)}-\left(b_{34}^{\prime \prime}\right)^{(6)}\left(\left(G_{35}\right)^{*}\right)\right]}
\end{aligned}
$$

Obviously, these values represent an equilibrium solution

\section{ASYMPTOTIC STABILITY ANALYSIS}

Theorem 4: If the conditions of the previous theorem are satisfied and if the functions $\left(a_{i}^{\prime \prime}\right)^{(1)}$ and $\left(b_{i}^{\prime \prime}\right)^{(1)}$ Belong to $C^{(1)}\left(\mathbb{R}_{+}\right)$then the above equilibrium point is asymptotically stable.

Proof:_Denote

\section{Definition of $\mathbb{G}_{i}, \mathbb{T}_{i}:-$}

$$
\begin{aligned}
& G_{i}=G_{i}^{*}+\mathbb{G}_{i} \quad, T_{i}=T_{i}^{*}+\mathbb{T}_{i} \\
& \frac{\partial\left(a_{14}^{\prime \prime}\right)^{(1)}}{\partial T_{14}}\left(T_{14}^{*}\right)=\left(q_{14}\right)^{(1)}, \frac{\partial\left(b_{i}^{\prime \prime}\right)^{(1)}}{\partial G_{j}}\left(G^{*}\right)=s_{i j}
\end{aligned}
$$

Then taking into account equations of global system neglecting the terms of power 2, we obtain

$$
\begin{aligned}
& \frac{d \mathbb{G}_{13}}{d t}=-\left(\left(a_{13}^{\prime}\right)^{(1)}+\left(p_{13}\right)^{(1)}\right) \mathbb{G}_{13}+\left(a_{13}\right)^{(1)} \mathbb{G}_{14}-\left(q_{13}\right)^{(1)} G_{13}^{*} \mathbb{T}_{14} \\
& \frac{d \mathbb{G}_{14}}{d t}=-\left(\left(a_{14}^{\prime}\right)^{(1)}+\left(p_{14}\right)^{(1)}\right) \mathbb{G}_{14}+\left(a_{14}\right)^{(1)} \mathbb{G}_{13}-\left(q_{14}\right)^{(1)} G_{14}^{*} \mathbb{T}_{14} \\
& \frac{d \mathbb{G}_{15}}{d t}=-\left(\left(a_{15}^{\prime}\right)^{(1)}+\left(p_{15}\right)^{(1)}\right) \mathbb{G}_{15}+\left(a_{15}\right)^{(1)} \mathbb{G}_{14}-\left(q_{15}\right)^{(1)} G_{15}^{*} \mathbb{T}_{14} \\
& \frac{d \mathbb{T}_{13}}{d t}=-\left(\left(b_{13}^{\prime}\right)^{(1)}-\left(r_{13}\right)^{(1)}\right) \mathbb{T}_{13}+\left(b_{13}\right)^{(1)} \mathbb{T}_{14}+\sum_{j=13}^{15}\left(s_{(13)(j)} T_{13}^{*} \mathbb{G}_{j}\right) \\
& \frac{d \mathbb{T}_{14}}{d t}=-\left(\left(b_{14}^{\prime}\right)^{(1)}-\left(r_{14}\right)^{(1)}\right) \mathbb{T}_{14}+\left(b_{14}\right)^{(1)} \mathbb{T}_{13}+\sum_{j=13}^{15}\left(s_{(14)(j)} T_{14}^{*} \mathbb{G}_{j}\right) \\
& \frac{d \mathbb{T}_{15}}{d t}=-\left(\left(b_{15}^{\prime}\right)^{(1)}-\left(r_{15}\right)^{(1)}\right) \mathbb{T}_{15}+\left(b_{15}\right)^{(1)} \mathbb{T}_{14}+\sum_{j=13}^{15}\left(s_{(15)(j)} T_{15}^{*} \mathbb{G}_{j}\right)
\end{aligned}
$$

If the conditions of the previous theorem are satisfied and if the functions $\left(a_{i}^{\prime \prime}\right)^{(2)}$ and $\left(b_{i}^{\prime \prime}\right)^{(2)}$ Belong to $\mathrm{C}^{(2)}\left(\mathbb{R}_{+}\right)$then the above equilibrium point is asymptotically stable

_Denote

Definition of $\mathbb{G}_{i}, \mathbb{T}_{i}$ :-

$\mathrm{G}_{i}=\mathrm{G}_{i}^{*}+\mathbb{G}_{i} \quad, \mathrm{~T}_{i}=\mathrm{T}_{i}^{*}+\mathbb{T}_{i}$ 
$\frac{\partial\left(a_{17}^{\prime \prime}\right)^{(2)}}{\partial \mathrm{T}_{17}}\left(\mathrm{~T}_{17}^{*}\right)=\left(q_{17}\right)^{(2)}, \frac{\partial\left(b_{i}^{\prime \prime}\right)^{(2)}}{\partial \mathrm{G}_{j}}\left(\left(G_{19}\right)^{*}\right)=s_{i j}$

taking into account equations (global) and neglecting the terms of power 2, we obtain

$\frac{\mathrm{d} \mathbb{G}_{16}}{\mathrm{dt}}=-\left(\left(a_{16}^{\prime}\right)^{(2)}+\left(p_{16}\right)^{(2)}\right) \mathbb{G}_{16}+\left(a_{16}\right)^{(2)} \mathbb{G}_{17}-\left(q_{16}\right)^{(2)} \mathrm{G}_{16}^{*} \mathbb{T}_{17}$

$\frac{\mathrm{d} \mathbb{G}_{17}}{\mathrm{dt}}=-\left(\left(a_{17}^{\prime}\right)^{(2)}+\left(p_{17}\right)^{(2)}\right) \mathbb{G}_{17}+\left(a_{17}\right)^{(2)} \mathbb{G}_{16}-\left(q_{17}\right)^{(2)} \mathrm{G}_{17}^{*} \mathbb{T}_{17}$

$\frac{\mathrm{d} \mathbb{G}_{18}}{\mathrm{dt}}=-\left(\left(a_{18}^{\prime}\right)^{(2)}+\left(p_{18}\right)^{(2)}\right) \mathbb{G}_{18}+\left(a_{18}\right)^{(2)} \mathbb{G}_{17}-\left(q_{18}\right)^{(2)} \mathrm{G}_{18}^{*} \mathbb{T}_{17}$

$\frac{\mathrm{d} \mathbb{T}_{16}}{\mathrm{dt}}=-\left(\left(b_{16}^{\prime}\right)^{(2)}-\left(r_{16}\right)^{(2)}\right) \mathbb{T}_{16}+\left(b_{16}\right)^{(2)} \mathbb{T}_{17}+\sum_{j=16}^{18}\left(s_{(16)(j)} \mathrm{T}_{16}^{*} \mathbb{G}_{j}\right)$

$\frac{\mathrm{d} \mathbb{T}_{17}}{\mathrm{dt}}=-\left(\left(b_{17}^{\prime}\right)^{(2)}-\left(r_{17}\right)^{(2)}\right) \mathbb{T}_{17}+\left(b_{17}\right)^{(2)} \mathbb{T}_{16}+\sum_{j=16}^{18}\left(s_{(17)(j)} \mathrm{T}_{17}^{*} \mathbb{G}_{j}\right)$

$\frac{\mathrm{d} \mathbb{T}_{18}}{\mathrm{dt}}=-\left(\left(b_{18}^{\prime}\right)^{(2)}-\left(r_{18}\right)^{(2)}\right) \mathbb{T}_{18}+\left(b_{18}\right)^{(2)} \mathbb{T}_{17}+\sum_{j=16}^{18}\left(s_{(18)(j)} \mathrm{T}_{18}^{*} \mathbb{G}_{j}\right)$

If the conditions of the previous theorem are satisfied and if the functions $\left(a_{i}^{\prime \prime}\right)^{(3)}$ and $\left(b_{i}^{\prime \prime}\right)^{(3)}$ Belong to $C^{(3)}\left(\mathbb{R}_{+}\right)$then the above equilibrium point is asymptotically stable.

-Denote

Definition of $\mathbb{G}_{i}, \mathbb{T}_{i}:-$

$$
\begin{aligned}
& G_{i}=G_{i}^{*}+\mathbb{G}_{i} \quad, T_{i}=T_{i}^{*}+\mathbb{T}_{i} \\
& \frac{\partial\left(a_{21}^{\prime \prime}\right)^{(3)}}{\partial T_{21}}\left(T_{21}^{*}\right)=\left(q_{21}\right)^{(3)}, \frac{\partial\left(b_{i}^{\prime \prime}\right)^{(3)}}{\partial G_{j}}\left(\left(G_{23}\right)^{*}\right)=s_{i j}
\end{aligned}
$$

Then taking into account equations (global) and neglecting the terms of power 2, we obtain

$\frac{d \mathbb{G}_{20}}{d t}=-\left(\left(a_{20}^{\prime}\right)^{(3)}+\left(p_{20}\right)^{(3)}\right) \mathbb{G}_{20}+\left(a_{20}\right)^{(3)} \mathbb{G}_{21}-\left(q_{20}\right)^{(3)} G_{20}^{*} \mathbb{T}_{21}$

$\frac{d \mathbb{G}_{21}}{d t}=-\left(\left(a_{21}^{\prime}\right)^{(3)}+\left(p_{21}\right)^{(3)}\right) \mathbb{G}_{21}+\left(a_{21}\right)^{(3)} \mathbb{G}_{20}-\left(q_{21}\right)^{(3)} G_{21}^{*} \mathbb{T}_{21}$

$\frac{d \mathbb{G}_{22}}{d t}=-\left(\left(a_{22}^{\prime}\right)^{(3)}+\left(p_{22}\right)^{(3)}\right) \mathbb{G}_{22}+\left(a_{22}\right)^{(3)} \mathbb{G}_{21}-\left(q_{22}\right)^{(3)} G_{22}^{*} \mathbb{T}_{21}$

$\frac{d \mathbb{T}_{20}}{d t}=-\left(\left(b_{20}^{\prime}\right)^{(3)}-\left(r_{20}\right)^{(3)}\right) \mathbb{T}_{20}+\left(b_{20}\right)^{(3)} \mathbb{T}_{21}+\sum_{j=20}^{22}\left(s_{(20)(j)} T_{20}^{*} \mathbb{G}_{j}\right)$

$\frac{d \mathbb{T}_{21}}{d t}=-\left(\left(b_{21}^{\prime}\right)^{(3)}-\left(r_{21}\right)^{(3)}\right) \mathbb{T}_{21}+\left(b_{21}\right)^{(3)} \mathbb{T}_{20}+\sum_{j=20}^{22}\left(s_{(21)(j)} T_{21}^{*} \mathbb{G}_{j}\right)$

$\frac{d \mathbb{T}_{22}}{d t}=-\left(\left(b_{22}^{\prime}\right)^{(3)}-\left(r_{22}\right)^{(3)}\right) \mathbb{T}_{22}+\left(b_{22}\right)^{(3)} \mathbb{T}_{21}+\sum_{j=20}^{22}\left(s_{(22)(j)} T_{22}^{*} \mathbb{G}_{j}\right)$

If the conditions of the previous theorem are satisfied and if the functions $\left(a_{i}^{\prime \prime}\right)^{(4)}$ and $\left(b_{i}^{\prime \prime}\right)^{(4)}$ Belong to $C^{(4)}\left(\mathbb{R}_{+}\right)$then the above equilibrium point is asymptotically stable.

Denote

Definition of $\mathbb{G}_{i}, \mathbb{T}_{i}:-$ 


$$
\begin{gathered}
G_{i}=G_{i}^{*}+\mathbb{G}_{i} \quad, T_{i}=T_{i}^{*}+\mathbb{T}_{i} \\
\frac{\partial\left(a_{25}^{\prime \prime}\right)^{(4)}}{\partial T_{25}}\left(T_{25}^{*}\right)=\left(q_{25}\right)^{(4)}, \frac{\partial\left(b_{i}^{\prime \prime}\right)^{(4)}}{\partial G_{j}}\left(\left(G_{27}\right)^{*}\right)=s_{i j}
\end{gathered}
$$

Then taking into account equations (global) and neglecting the terms of power 2, we obtain

$$
\begin{aligned}
& \frac{d \mathbb{G}_{24}}{d t}=-\left(\left(a_{24}^{\prime}\right)^{(4)}+\left(p_{24}\right)^{(4)}\right) \mathbb{G}_{24}+\left(a_{24}\right)^{(4)} \mathbb{G}_{25}-\left(q_{24}\right)^{(4)} G_{24}^{*} \mathbb{T}_{25} \\
& \frac{d \mathbb{G}_{25}}{d t}=-\left(\left(a_{25}^{\prime}\right)^{(4)}+\left(p_{25}\right)^{(4)}\right) \mathbb{G}_{25}+\left(a_{25}\right)^{(4)} \mathbb{G}_{24}-\left(q_{25}\right)^{(4)} G_{25}^{*} \mathbb{T}_{25} \\
& \frac{d \mathbb{G}_{26}}{d t}=-\left(\left(a_{26}^{\prime}\right)^{(4)}+\left(p_{26}\right)^{(4)}\right) \mathbb{G}_{26}+\left(a_{26}\right)^{(4)} \mathbb{G}_{25}-\left(q_{26}\right)^{(4)} G_{26}^{*} \mathbb{T}_{25} \\
& \frac{d \mathbb{T}_{24}}{d t}=-\left(\left(b_{24}^{\prime}\right)^{(4)}-\left(r_{24}\right)^{(4)}\right) \mathbb{T}_{24}+\left(b_{24}\right)^{(4)} \mathbb{T}_{25}+\sum_{j=24}^{26}\left(s_{(24)(j)} T_{24}^{*} \mathbb{G}_{j}\right) \\
& \frac{d \mathbb{T}_{25}}{d t}=-\left(\left(b_{25}^{\prime}\right)^{(4)}-\left(r_{25}\right)^{(4)}\right) \mathbb{T}_{25}+\left(b_{25}\right)^{(4)} \mathbb{T}_{24}+\sum_{j=24}^{26}\left(s_{(25)(j)} T_{25}^{*} \mathbb{G}_{j}\right) \\
& \frac{d \mathbb{T}_{26}}{d t}=-\left(\left(b_{26}^{\prime}\right)^{(4)}-\left(r_{26}\right)^{(4)}\right) \mathbb{T}_{26}+\left(b_{26}\right)^{(4)} \mathbb{T}_{25}+\sum_{j=24}^{26}\left(s_{(26)(j)} T_{26}^{*} \mathbb{G}_{j}\right)
\end{aligned}
$$

If the conditions of the previous theorem are satisfied and if the functions $\left(a_{i}^{\prime \prime}\right)^{(5)}$ and $\left(b_{i}^{\prime \prime}\right)^{(5)}$ Belong to $C^{(5)}\left(\mathbb{R}_{+}\right)$then the above equilibrium point is asymptotically stable.

\section{-Denote}

Definition of $\mathbb{G}_{i}, \mathbb{T}_{i}:-$

$$
\begin{gathered}
G_{i}=G_{i}^{*}+\mathbb{G}_{i} \quad, T_{i}=T_{i}^{*}+\mathbb{T}_{i} \\
\frac{\partial\left(a_{29}^{\prime \prime}\right)^{(5)}}{\partial T_{29}}\left(T_{29}^{*}\right)=\left(q_{29}\right)^{(5)}, \frac{\partial\left(b_{i}^{\prime \prime}\right)^{(5)}}{\partial G_{j}}\left(\left(G_{31}\right)^{*}\right)=s_{i j}
\end{gathered}
$$

Then taking into account equations(global) and neglecting the terms of power 2, we obtain

$$
\begin{aligned}
& \frac{d \mathbb{G}_{28}}{d t}=-\left(\left(a_{28}^{\prime}\right)^{(5)}+\left(p_{28}\right)^{(5)}\right) \mathbb{G}_{28}+\left(a_{28}\right)^{(5)} \mathbb{G}_{29}-\left(q_{28}\right)^{(5)} G_{28}^{*} \mathbb{T}_{29} \\
& \frac{d \mathbb{G}_{29}}{d t}=-\left(\left(a_{29}^{\prime}\right)^{(5)}+\left(p_{29}\right)^{(5)}\right) \mathbb{G}_{29}+\left(a_{29}\right)^{(5)} \mathbb{G}_{28}-\left(q_{29}\right)^{(5)} G_{29}^{*} \mathbb{T}_{29} \\
& \frac{d \mathbb{G}_{30}}{d t}=-\left(\left(a_{30}^{\prime}\right)^{(5)}+\left(p_{30}\right)^{(5)}\right) \mathbb{G}_{30}+\left(a_{30}\right)^{(5)} \mathbb{G}_{29}-\left(q_{30}\right)^{(5)} G_{30}^{*} \mathbb{T}_{29} \\
& \frac{d \mathbb{T}_{28}}{d t}=-\left(\left(b_{28}^{\prime}\right)^{(5)}-\left(r_{28}\right)^{(5)}\right) \mathbb{T}_{28}+\left(b_{28}\right)^{(5)} \mathbb{T}_{29}+\sum_{j=28}^{30}\left(s_{(28)(j)} T_{28}^{*} \mathbb{G}_{j}\right) \\
& \frac{d \mathbb{T}_{29}}{d t}=-\left(\left(b_{29}^{\prime}\right)^{(5)}-\left(r_{29}\right)^{(5)}\right) \mathbb{T}_{29}+\left(b_{29}\right)^{(5)} \mathbb{T}_{28}+\sum_{j=28}^{30}\left(s_{(29)(j)} T_{29}^{*} \mathbb{G}_{j}\right) \\
& \frac{d \mathbb{T}_{30}}{d t}=-\left(\left(b_{30}^{\prime}\right)^{(5)}-\left(r_{30}\right)^{(5)}\right) \mathbb{T}_{30}+\left(b_{30}\right)^{(5)} \mathbb{T}_{29}+\sum_{j=28}^{30}\left(s_{(30)(j)} T_{30}^{*} \mathbb{G}_{j}\right)
\end{aligned}
$$

If the conditions of the previous theorem are satisfied and if the functions $\left(a_{i}^{\prime \prime}\right)^{(6)}$ and $\left(b_{i}^{\prime \prime}\right)^{(6)}$ Belong to $C^{(6)}\left(\mathbb{R}_{+}\right)$then the above equilibrium point is asymptotically stable.

\section{Denote}


Definition of $\mathbb{G}_{i}, \mathbb{T}_{i}:-$

$$
\begin{aligned}
& G_{i}=G_{i}^{*}+\mathbb{G}_{i} \quad, T_{i}=T_{i}^{*}+\mathbb{T}_{i} \\
& \frac{\partial\left(a_{33}^{\prime \prime}\right)^{(6)}}{\partial T_{33}}\left(T_{33}^{*}\right)=\left(q_{33}\right)^{(6)}, \frac{\partial\left(b_{i}^{\prime \prime}\right)^{(6)}}{\partial G_{j}}\left(\left(G_{35}\right)^{*}\right)=s_{i j}
\end{aligned}
$$

Then taking into account equations(global) and neglecting the terms of power 2, we obtain

$$
\begin{aligned}
& \frac{d \mathbb{G}_{32}}{d t}=-\left(\left(a_{32}^{\prime}\right)^{(6)}+\left(p_{32}\right)^{(6)}\right) \mathbb{G}_{32}+\left(a_{32}\right)^{(6)} \mathbb{G}_{33}-\left(q_{32}\right)^{(6)} G_{32}^{*} \mathbb{T}_{33} \\
& \frac{d \mathbb{G}_{33}}{d t}=-\left(\left(a_{33}^{\prime}\right)^{(6)}+\left(p_{33}\right)^{(6)}\right) \mathbb{G}_{33}+\left(a_{33}\right)^{(6)} \mathbb{G}_{32}-\left(q_{33}\right)^{(6)} G_{33}^{*} \mathbb{T}_{33} \\
& \frac{d \mathbb{G}_{34}}{d t}=-\left(\left(a_{34}^{\prime}\right)^{(6)}+\left(p_{34}\right)^{(6)}\right) \mathbb{G}_{34}+\left(a_{34}\right)^{(6)} \mathbb{G}_{33}-\left(q_{34}\right)^{(6)} G_{34}^{*} \mathbb{T}_{33} \\
& \frac{d \mathbb{T}_{32}}{d t}=-\left(\left(b_{32}^{\prime}\right)^{(6)}-\left(r_{32}\right)^{(6)}\right) \mathbb{T}_{32}+\left(b_{32}\right)^{(6)} \mathbb{T}_{33}+\sum_{j=32}^{34}\left(s_{(32)(j)} T_{32}^{*} \mathbb{G}_{j}\right) \\
& \frac{d \mathbb{T}_{33}}{d t}=-\left(\left(b_{33}^{\prime}\right)^{(6)}-\left(r_{33}\right)^{(6)}\right) \mathbb{T}_{33}+\left(b_{33}\right)^{(6)} \mathbb{T}_{32}+\sum_{j=32}^{34}\left(s_{(33)(j)} T_{33}^{*} \mathbb{G}_{j}\right) \\
& \frac{d \mathbb{T}_{34}}{d t}=-\left(\left(b_{34}^{\prime}\right)^{(6)}-\left(r_{34}\right)^{(6)}\right) \mathbb{T}_{34}+\left(b_{34}\right)^{(6)} \mathbb{T}_{33}+\sum_{j=32}^{34}\left(s_{(34)(j)} T_{34}^{*} \mathbb{G}_{j}\right)
\end{aligned}
$$

The characteristic equation of this system is

$$
\begin{aligned}
& \left((\lambda)^{(1)}+\left(b_{15}^{\prime}\right)^{(1)}-\left(r_{15}\right)^{(1)}\right)\left\{\left((\lambda)^{(1)}+\left(a_{15}^{\prime}\right)^{(1)}+\left(p_{15}\right)^{(1)}\right)\right. \\
& {\left[\left(\left((\lambda)^{(1)}+\left(a_{13}^{\prime}\right)^{(1)}+\left(p_{13}\right)^{(1)}\right)\left(q_{14}\right)^{(1)} G_{14}^{*}+\left(a_{14}\right)^{(1)}\left(q_{13}\right)^{(1)} G_{13}^{*}\right)\right]} \\
& \left(\left((\lambda)^{(1)}+\left(b_{13}^{\prime}\right)^{(1)}-\left(r_{13}\right)^{(1)}\right) s_{(14),(14)} T_{14}^{*}+\left(b_{14}\right)^{(1)} s_{(13),(14)} T_{14}^{*}\right) \\
& +\left(\left((\lambda)^{(1)}+\left(a_{14}^{\prime}\right)^{(1)}+\left(p_{14}\right)^{(1)}\right)\left(q_{13}\right)^{(1)} G_{13}^{*}+\left(a_{13}\right)^{(1)}\left(q_{14}\right)^{(1)} G_{14}^{*}\right) \\
& \left(\left((\lambda)^{(1)}+\left(b_{13}^{\prime}\right)^{(1)}-\left(r_{13}\right)^{(1)}\right) s_{(14),(13)} T_{14}^{*}+\left(b_{14}\right)^{(1)} s_{(13),(13)} T_{13}^{*}\right) \\
& \left(\left((\lambda)^{(1)}\right)^{2}+\left(\left(a_{13}^{\prime}\right)^{(1)}+\left(a_{14}^{\prime}\right)^{(1)}+\left(p_{13}\right)^{(1)}+\left(p_{14}\right)^{(1)}\right)(\lambda)^{(1)}\right) \\
& \left(\left((\lambda)^{(1)}\right)^{2}+\left(\left(b_{13}^{\prime}\right)^{(1)}+\left(b_{14}^{\prime}\right)^{(1)}-\left(r_{13}\right)^{(1)}+\left(r_{14}\right)^{(1)}\right)(\lambda)^{(1)}\right) \\
& +\left(\left((\lambda)^{(1)}\right)^{2}+\left(\left(a_{13}^{\prime}\right)^{(1)}+\left(a_{14}^{\prime}\right)^{(1)}+\left(p_{13}\right)^{(1)}+\left(p_{14}\right)^{(1)}\right)(\lambda)^{(1)}\right)\left(q_{15}\right)^{(1)} G_{15} \\
& +\left((\lambda)^{(1)}+\left(a_{13}^{\prime}\right)^{(1)}+\left(p_{13}\right)^{(1)}\right)\left(\left(a_{15}\right)^{(1)}\left(q_{14}\right)^{(1)} G_{14}^{*}+\left(a_{14}\right)^{(1)}\left(a_{15}\right)^{(1)}\left(q_{13}\right)^{(1)} G_{13}^{*}\right) \\
& \left.\left(\left((\lambda)^{(1)}+\left(b_{13}^{\prime}\right)^{(1)}-\left(r_{13}\right)^{(1)}\right) s_{(14),(15)} T_{14}^{*}+\left(b_{14}\right)^{(1)} s_{(13),(15)} T_{13}^{*}\right)\right\}=0 \\
& + \\
& \left((\lambda)^{(2)}+\left(b_{18}^{\prime}\right)^{(2)}-\left(r_{18}\right)^{(2)}\right)\left\{\left((\lambda)^{(2)}+\left(a_{18}^{\prime}\right)^{(2)}+\left(p_{18}\right)^{(2)}\right)\right. \\
& {\left[\left(\left((\lambda)^{(2)}+\left(a_{16}^{\prime}\right)^{(2)}+\left(p_{16}\right)^{(2)}\right)\left(q_{17}\right)^{(2)} G_{17}^{*}+\left(a_{17}\right)^{(2)}\left(q_{16}\right)^{(2)} G_{16}^{*}\right)\right]}
\end{aligned}
$$




$$
\begin{aligned}
& \left(\left((\lambda)^{(2)}+\left(b_{16}^{\prime}\right)^{(2)}-\left(r_{16}\right)^{(2)}\right) s_{(17),(17)} \mathrm{T}_{17}^{*}+\left(b_{17}\right)^{(2)} s_{(16),(17)} \mathrm{T}_{17}^{*}\right) \\
& +\left(\left((\lambda)^{(2)}+\left(a_{17}^{\prime}\right)^{(2)}+\left(p_{17}\right)^{(2)}\right)\left(q_{16}\right)^{(2)} \mathrm{G}_{16}^{*}+\left(a_{16}\right)^{(2)}\left(q_{17}\right)^{(2)} \mathrm{G}_{17}^{*}\right) \\
& \left(\left((\lambda)^{(2)}+\left(b_{16}^{\prime}\right)^{(2)}-\left(r_{16}\right)^{(2)}\right) s_{(17),(16)} \mathrm{T}_{17}^{*}+\left(b_{17}\right)^{(2)} s_{(16),(16)} \mathrm{T}_{16}^{*}\right) \\
& \left(\left((\lambda)^{(2)}\right)^{2}+\left(\left(a_{16}^{\prime}\right)^{(2)}+\left(a_{17}^{\prime}\right)^{(2)}+\left(p_{16}\right)^{(2)}+\left(p_{17}\right)^{(2)}\right)(\lambda)^{(2)}\right) \\
& \left(\left((\lambda)^{(2)}\right)^{2}+\left(\left(b_{16}^{\prime}\right)^{(2)}+\left(b_{17}^{\prime}\right)^{(2)}-\left(r_{16}\right)^{(2)}+\left(r_{17}\right)^{(2)}\right)(\lambda)^{(2)}\right) \\
& +\left(\left((\lambda)^{(2)}\right)^{2}+\left(\left(a_{16}^{\prime}\right)^{(2)}+\left(a_{17}^{\prime}\right)^{(2)}+\left(p_{16}\right)^{(2)}+\left(p_{17}\right)^{(2)}\right)(\lambda)^{(2)}\right)\left(q_{18}\right)^{(2)} \mathrm{G}_{18} \\
& +\left((\lambda)^{(2)}+\left(a_{16}^{\prime}\right)^{(2)}+\left(p_{16}\right)^{(2)}\right)\left(\left(a_{18}\right)^{(2)}\left(q_{17}\right)^{(2)} \mathrm{G}_{17}^{*}+\left(a_{17}\right)^{(2)}\left(a_{18}\right)^{(2)}\left(q_{16}\right)^{(2)} \mathrm{G}_{16}^{*}\right) \\
& \left.\left(\left((\lambda)^{(2)}+\left(b_{16}^{\prime}\right)^{(2)}-\left(r_{16}\right)^{(2)}\right) s_{(17),(18)} \mathrm{T}_{17}^{*}+\left(b_{17}\right)^{(2)} s_{(16),(18)} \mathrm{T}_{16}^{*}\right)\right\}=0 \\
& \left((\lambda)^{(3)}+\left(b_{22}^{\prime}\right)^{(3)}-\left(r_{22}\right)^{(3)}\right)\left\{\left((\lambda)^{(3)}+\left(a_{22}^{\prime}\right)^{(3)}+\left(p_{22}\right)^{(3)}\right)\right. \\
& {\left[\left(\left((\lambda)^{(3)}+\left(a_{20}^{\prime}\right)^{(3)}+\left(p_{20}\right)^{(3)}\right)\left(q_{21}\right)^{(3)} G_{21}^{*}+\left(a_{21}\right)^{(3)}\left(q_{20}\right)^{(3)} G_{20}^{*}\right)\right]} \\
& \left(\left((\lambda)^{(3)}+\left(b_{20}^{\prime}\right)^{(3)}-\left(r_{20}\right)^{(3)}\right) s_{(21),(21)} T_{21}^{*}+\left(b_{21}\right)^{(3)} s_{(20),(21)} T_{21}^{*}\right) \\
& +\left(\left((\lambda)^{(3)}+\left(a_{21}^{\prime}\right)^{(3)}+\left(p_{21}\right)^{(3)}\right)\left(q_{20}\right)^{(3)} G_{20}^{*}+\left(a_{20}\right)^{(3)}\left(q_{21}\right)^{(1)} G_{21}^{*}\right) \\
& \left(\left((\lambda)^{(3)}+\left(b_{20}^{\prime}\right)^{(3)}-\left(r_{20}\right)^{(3)}\right) s_{(21),(20)} T_{21}^{*}+\left(b_{21}\right)^{(3)} s_{(20),(20)} T_{20}^{*}\right) \\
& \left(\left((\lambda)^{(3)}\right)^{2}+\left(\left(a_{20}^{\prime}\right)^{(3)}+\left(a_{21}^{\prime}\right)^{(3)}+\left(p_{20}\right)^{(3)}+\left(p_{21}\right)^{(3)}\right)(\lambda)^{(3)}\right) \\
& \left(\left((\lambda)^{(3)}\right)^{2}+\left(\left(b_{20}^{\prime}\right)^{(3)}+\left(b_{21}^{\prime}\right)^{(3)}-\left(r_{20}\right)^{(3)}+\left(r_{21}\right)^{(3)}\right)(\lambda)^{(3)}\right) \\
& +\left(\left((\lambda)^{(3)}\right)^{2}+\left(\left(a_{20}^{\prime}\right)^{(3)}+\left(a_{21}^{\prime}\right)^{(3)}+\left(p_{20}\right)^{(3)}+\left(p_{21}\right)^{(3)}\right)(\lambda)^{(3)}\right)\left(q_{22}\right)^{(3)} G_{22} \\
& +\left((\lambda)^{(3)}+\left(a_{20}^{\prime}\right)^{(3)}+\left(p_{20}\right)^{(3)}\right)\left(\left(a_{22}\right)^{(3)}\left(q_{21}\right)^{(3)} G_{21}^{*}+\left(a_{21}\right)^{(3)}\left(a_{22}\right)^{(3)}\left(q_{20}\right)^{(3)} G_{20}^{*}\right) \\
& \left.\left(\left((\lambda)^{(3)}+\left(b_{20}^{\prime}\right)^{(3)}-\left(r_{20}\right)^{(3)}\right) s_{(21),(22)} T_{21}^{*}+\left(b_{21}\right)^{(3)} s_{(20),(22)} T_{20}^{*}\right)\right\}=0 \\
& \left((\lambda)^{(4)}+\left(b_{26}^{\prime}\right)^{(4)}-\left(r_{26}\right)^{(4)}\right)\left\{\left((\lambda)^{(4)}+\left(a_{26}^{\prime}\right)^{(4)}+\left(p_{26}\right)^{(4)}\right)\right. \\
& {\left[\left(\left((\lambda)^{(4)}+\left(a_{24}^{\prime}\right)^{(4)}+\left(p_{24}\right)^{(4)}\right)\left(q_{25}\right)^{(4)} G_{25}^{*}+\left(a_{25}\right)^{(4)}\left(q_{24}\right)^{(4)} G_{24}^{*}\right)\right]} \\
& \left(\left((\lambda)^{(4)}+\left(b_{24}^{\prime}\right)^{(4)}-\left(r_{24}\right)^{(4)}\right) s_{(25),(25)} T_{25}^{*}+\left(b_{25}\right)^{(4)} s_{(24),(25)} T_{25}^{*}\right)
\end{aligned}
$$




$$
\begin{aligned}
& +\left(\left((\lambda)^{(4)}+\left(a_{25}^{\prime}\right)^{(4)}+\left(p_{25}\right)^{(4)}\right)\left(q_{24}\right)^{(4)} G_{24}^{*}+\left(a_{24}\right)^{(4)}\left(q_{25}\right)^{(4)} G_{25}^{*}\right) \\
& \left(\left((\lambda)^{(4)}+\left(b_{24}^{\prime}\right)^{(4)}-\left(r_{24}\right)^{(4)}\right) s_{(25),(24)} T_{25}^{*}+\left(b_{25}\right)^{(4)} s_{(24),(24)} T_{24}^{*}\right) \\
& \left(\left((\lambda)^{(4)}\right)^{2}+\left(\left(a_{24}^{\prime}\right)^{(4)}+\left(a_{25}^{\prime}\right)^{(4)}+\left(p_{24}\right)^{(4)}+\left(p_{25}\right)^{(4)}\right)(\lambda)^{(4)}\right) \\
& \left(\left((\lambda)^{(4)}\right)^{2}+\left(\left(b_{24}^{\prime}\right)^{(4)}+\left(b_{25}^{\prime}\right)^{(4)}-\left(r_{24}\right)^{(4)}+\left(r_{25}\right)^{(4)}\right)(\lambda)^{(4)}\right) \\
& +\left(\left((\lambda)^{(4)}\right)^{2}+\left(\left(a_{24}^{\prime}\right)^{(4)}+\left(a_{25}^{\prime}\right)^{(4)}+\left(p_{24}\right)^{(4)}+\left(p_{25}\right)^{(4)}\right)(\lambda)^{(4)}\right)\left(q_{26}\right)^{(4)} G_{26} \\
& +\left((\lambda)^{(4)}+\left(a_{24}^{\prime}\right)^{(4)}+\left(p_{24}\right)^{(4)}\right)\left(\left(a_{26}\right)^{(4)}\left(q_{25}\right)^{(4)} G_{25}^{*}+\left(a_{25}\right)^{(4)}\left(a_{26}\right)^{(4)}\left(q_{24}\right)^{(4)} G_{24}^{*}\right) \\
& \left.\left(\left((\lambda)^{(4)}+\left(b_{24}^{\prime}\right)^{(4)}-\left(r_{24}\right)^{(4)}\right) s_{(25),(26)} T_{25}^{*}+\left(b_{25}\right)^{(4)} s_{(24),(26)} T_{24}^{*}\right)\right\}=0 \\
& \left((\lambda)^{(5)}+\left(b_{30}^{\prime}\right)^{(5)}-\left(r_{30}\right)^{(5)}\right)\left\{\left((\lambda)^{(5)}+\left(a_{30}^{\prime}\right)^{(5)}+\left(p_{30}\right)^{(5)}\right)\right. \\
& {\left[\left(\left((\lambda)^{(5)}+\left(a_{28}^{\prime}\right)^{(5)}+\left(p_{28}\right)^{(5)}\right)\left(q_{29}\right)^{(5)} G_{29}^{*}+\left(a_{29}\right)^{(5)}\left(q_{28}\right)^{(5)} G_{28}^{*}\right)\right]} \\
& \left(\left((\lambda)^{(5)}+\left(b_{28}^{\prime}\right)^{(5)}-\left(r_{28}\right)^{(5)}\right) s_{(29),(29)} T_{29}^{*}+\left(b_{29}\right)^{(5)} s_{(28),(29)} T_{29}^{*}\right) \\
& +\left(\left((\lambda)^{(5)}+\left(a_{29}^{\prime}\right)^{(5)}+\left(p_{29}\right)^{(5)}\right)\left(q_{28}\right)^{(5)} G_{28}^{*}+\left(a_{28}\right)^{(5)}\left(q_{29}\right)^{(5)} G_{29}^{*}\right) \\
& \left(\left((\lambda)^{(5)}+\left(b_{28}^{\prime}\right)^{(5)}-\left(r_{28}\right)^{(5)}\right) s_{(29),(28)} T_{29}^{*}+\left(b_{29}\right)^{(5)} s_{(28),(28)} T_{28}^{*}\right) \\
& \left(\left((\lambda)^{(5)}\right)^{2}+\left(\left(a_{28}^{\prime}\right)^{(5)}+\left(a_{29}^{\prime}\right)^{(5)}+\left(p_{28}\right)^{(5)}+\left(p_{29}\right)^{(5)}\right)(\lambda)^{(5)}\right) \\
& \left(\left((\lambda)^{(5)}\right)^{2}+\left(\left(b_{28}^{\prime}\right)^{(5)}+\left(b_{29}^{\prime}\right)^{(5)}-\left(r_{28}\right)^{(5)}+\left(r_{29}\right)^{(5)}\right)(\lambda)^{(5)}\right) \\
& +\left(\left((\lambda)^{(5)}\right)^{2}+\left(\left(a_{28}^{\prime}\right)^{(5)}+\left(a_{29}^{\prime}\right)^{(5)}+\left(p_{28}\right)^{(5)}+\left(p_{29}\right)^{(5)}\right)(\lambda)^{(5)}\right)\left(q_{30}\right)^{(5)} G_{30} \\
& +\left((\lambda)^{(5)}+\left(a_{28}^{\prime}\right)^{(5)}+\left(p_{28}\right)^{(5)}\right)\left(\left(a_{30}\right)^{(5)}\left(q_{29}\right)^{(5)} G_{29}^{*}+\left(a_{29}\right)^{(5)}\left(a_{30}\right)^{(5)}\left(q_{28}\right)^{(5)} G_{28}^{*}\right) \\
& \left.\left(\left((\lambda)^{(5)}+\left(b_{28}^{\prime}\right)^{(5)}-\left(r_{28}\right)^{(5)}\right) s_{(29),(30)} T_{29}^{*}+\left(b_{29}\right)^{(5)} s_{(28),(30)} T_{28}^{*}\right)\right\}=0 \\
& \left((\lambda)^{(6)}+\left(b_{34}^{\prime}\right)^{(6)}-\left(r_{34}\right)^{(6)}\right)\left\{\left((\lambda)^{(6)}+\left(a_{34}^{\prime}\right)^{(6)}+\left(p_{34}\right)^{(6)}\right)\right. \\
& {\left[\left(\left((\lambda)^{(6)}+\left(a_{32}^{\prime}\right)^{(6)}+\left(p_{32}\right)^{(6)}\right)\left(q_{33}\right)^{(6)} G_{33}^{*}+\left(a_{33}\right)^{(6)}\left(q_{32}\right)^{(6)} G_{32}^{*}\right)\right]} \\
& \left(\left((\lambda)^{(6)}+\left(b_{32}^{\prime}\right)^{(6)}-\left(r_{32}\right)^{(6)}\right) s_{(33),(33)} T_{33}^{*}+\left(b_{33}\right)^{(6)} s_{(32),(33)} T_{33}^{*}\right) \\
& +\left(\left((\lambda)^{(6)}+\left(a_{33}^{\prime}\right)^{(6)}+\left(p_{33}\right)^{(6)}\right)\left(q_{32}\right)^{(6)} G_{32}^{*}+\left(a_{32}\right)^{(6)}\left(q_{33}\right)^{(6)} G_{33}^{*}\right)
\end{aligned}
$$




$$
\begin{aligned}
& \left(\left((\lambda)^{(6)}+\left(b_{32}^{\prime}\right)^{(6)}-\left(r_{32}\right)^{(6)}\right) s_{(33),(32)} T_{33}^{*}+\left(b_{33}\right)^{(6)} s_{(32),(32)} T_{32}^{*}\right) \\
& \left(\left((\lambda)^{(6)}\right)^{2}+\left(\left(a_{32}^{\prime}\right)^{(6)}+\left(a_{33}^{\prime}\right)^{(6)}+\left(p_{32}\right)^{(6)}+\left(p_{33}\right)^{(6)}\right)(\lambda)^{(6)}\right) \\
& \left(\left((\lambda)^{(6)}\right)^{2}+\left(\left(b_{32}^{\prime}\right)^{(6)}+\left(b_{33}^{\prime}\right)^{(6)}-\left(r_{32}\right)^{(6)}+\left(r_{33}\right)^{(6)}\right)(\lambda)^{(6)}\right) \\
& +\left(\left((\lambda)^{(6)}\right)^{2}+\left(\left(a_{32}^{\prime}\right)^{(6)}+\left(a_{33}^{\prime}\right)^{(6)}+\left(p_{32}\right)^{(6)}+\left(p_{33}\right)^{(6)}\right)(\lambda)^{(6)}\right)\left(q_{34}\right)^{(6)} G_{34} \\
& +\left((\lambda)^{(6)}+\left(a_{32}^{\prime}\right)^{(6)}+\left(p_{32}\right)^{(6)}\right)\left(\left(a_{34}\right)^{(6)}\left(q_{33}\right)^{(6)} G_{33}^{*}+\left(a_{33}\right)^{(6)}\left(a_{34}\right)^{(6)}\left(q_{32}\right)^{(6)} G_{32}^{*}\right) \\
& \left.\left(\left((\lambda)^{(6)}+\left(b_{32}^{\prime}\right)^{(6)}-\left(r_{32}\right)^{(6)}\right) s_{(33),(34)} T_{33}^{*}+\left(b_{33}\right)^{(6)} s_{(32),(34)} T_{32}^{*}\right)\right\}=0
\end{aligned}
$$

And as one sees, all the coefficients are positive. It follows that all the roots have negative real part, and this proves the theorem.

\section{Acknowledgments:}

The introduction is a collection of information from various articles, Books, News Paper reports, Home Pages Of authors, Journal Reviews, the internet including Wikipedia. We acknowledge all authors who have contributed to the same. In the eventuality of the fact that there has been any act of omission on the part of the authors, We regret with great deal of compunction, contrition, and remorse. As Newton said, it is only because erudite and eminent people allowed one to piggy ride on their backs; probably an attempt has been made to look slightly further. Once again, it is stated that the references are only illustrative and not comprehensive.

\section{REFERENCES}

[1] Garey, Michael R.; Johnson, D. S. (1979). Victor Klee. Ed. Computers and Intractability: A Guide to the Theory of NP-Completeness. A Series of Books in the Mathematical Sciences. W. H. Freeman and Co... pp. x+338. ISBN 0-7167-1045-5. MR 519066.

[2] Agrawal, M.; Allender, E.; Rudich, Steven (1998). "Reductions in Circuit Complexity: An Isomorphism Theorem and a Gap Theorem". Journal of Computer and System Sciences (Boston, MA: Academic Press) 57 (2): 127143. DOI:10.1006/jcss.1998.1583. ISSN 1090-2724

[3] Agrawal, M.; Allender, E.; Impagliazzo, R.; Pitassi, T.; Rudich, Steven (2001). "Reducing the complexity of reductions". Computational Complexity (Birkhäuser Basel) 10 (2): 117-138.DOI:10.1007/s00037-001-8191-1. ISSN 1016-3328

[4] Don Knuth, Tracy Larrabee, and Paul M. Roberts, Mathematical Writing § 25, MAA Notes No. 14, MAA, 1989 (also Stanford Technical Report, 1987)

[5] Knuth, D. F. (1974). "A terminological proposal". SIGACT News 6 (1): 12-18. DOI:10.1145/1811129.1811130. Retrieved 2010 08-28.

[6] http://www.nature.com/news/2000/000113/full/news000113-10.html

[7] Garey, M.R.; Johnson, D.S. (1979). Computers and Intractability: A Guide to the Theory of NP-Completeness. New York: W.H. Freeman. ISBN 0-7167-1045-5. This book is a classic, developing the theory, and then cataloguing many NP-Complete problems

[8] Cook, S.A. (1971). "The complexity of theorem proving procedures". Proceedings, Third Annual ACM Symposium on the Theory of Computing, ACM, New York. pp. 151-158.DOI:10.1145/800157.805047.

[9] Dunne, P.E. "An annotated list of selected NP-complete problems". COMP202, Dept. of Computer Science, University of Liverpool. Retrieved 2008-06-21.

[10] Crescenzi, P.; Kann, V.; Halldórsson, M.; Karpinski, M.; Woeginger, G. "A compendium of NP optimization problems". KTH NADA, Stockholm. Retrieved 2008-06-21.

[11] Dahlke, K. "NP-complete problems". Math Reference Project. Retrieved 2008-06-21.

[12] Karlsson, R. "Lecture 8: NP-complete problems" (PDF). Dept. of Computer Science, Lund University, Sweden. Retrieved 2008-0621.[dead link]

[13] Sun, H.M. "The theory of NP-completeness" (PPT). Information Security Laboratory, Dept. of Computer Science, National Tsing Hua University, Hsinchu City, Taiwan. Retrieved 2008-06-21.

[14] Jiang, J.R. "The theory of NP-completeness" (PPT). Dept. of Computer Science and Information Engineering, National Central University, Jhongli City, Taiwan. Retrieved 2008-06-21.

[15] Cormen, T.H.; Leiserson, C.E., Rivest, R.L.; Stein, C. (2001). Introduction to Algorithms (2nd ed.). MIT Press and McGraw-Hill Chapter 34: NP-Completeness, pp. 966-1021.ISBN 0-262-03293-7.

[16] Sipser, M. (1997). Introduction to the Theory of Computation. PWS Publishing. Sections 7.4-7.5 (NP-completeness, Additional NP-complete Problems), pp. 248-271. ISBN 0-534-94728-X.

[17] Papadimitriou, C. (1994). Computational Complexity (1st ed.). Addison Wesley. Chapter 9 (NP-complete problems), pp. 181218. ISBN 0-201-53082-1.

[18] Computational Complexity of Games and Puzzles 
First Author: ${ }^{1}$ Mr. K. N.Prasanna Kumar has three doctorates one each in Mathematics, Economics, Political Science. Thesis was based on Mathematical Modeling. He was recently awarded D.litt., for his work on 'Mathematical Models in Political Science'--- Department of studies in Mathematics, Kuvempu University, Shimoga, Karnataka, India

Second Author: ${ }^{2}$ Prof. B.S Kiranagi is the Former Chairman of the Department of Studies in Mathematics, Manasa Gangotri and present Professor Emeritus of UGC in the Department. Professor Kiranagi has guided over 25 students and he has received many encomiums and laurels for his contribution to Co homology Groups and Mathematical Sciences. Known for his prolific writing, and one of the senior most Professors of the country, he has over 150 publications to his credit. A prolific writer and a prodigious thinker, he has to his credit several books on Lie Groups, Co Homology Groups, and other mathematical application topics, and excellent publication history.-- UGC Emeritus Professor (Department of studies in Mathematics), Manasagangotri, University of Mysore, Karnataka, India

Third Author: ${ }^{3}$ Prof. C.S. Bagewadi is the present Chairman of Department of Mathematics and Department of Studies in Computer Science and has guided over 25 students. He has published articles in both national and international journals. Professor Bagewadi specializes in Differential Geometry and its wide-ranging ramifications. He has to his credit more than 159 research papers. Several Books on Differential Geometry, Differential Equations are coauthored by him--Chairman, Department of studies in Mathematics and Computer science, Jnanasahyadri Kuvempu University, Shankarghatta, Shimoga district, Karnataka, India 